\title{
Utilization of Ash From Municipal Solid Waste Combustion
}

\section{Final Report Phase I}

Colin M. Jones

R. Michael Hartman

Denton Kort

Neil Rapues

Honolulu, Hawaii

NREL technical monitor: Philip Shepherd

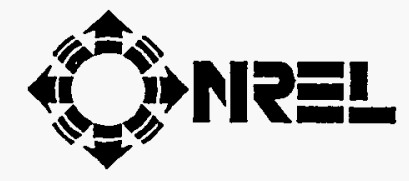

National Renewable Energy Laboratory 1617 Cole Boulevard Golden, Colorado 80401-3393

A national laboratory ofthe U.S. Department of Energy Managed by Midwest Research Institute for the Department of Energy under contract No. DE-AC36-83CH10093

Prepared under Subcontract No. XAR-3-1322

September 1994 


\section{NOTICE}

This report was prepared as an account of work sponsored by an agency of the United States government. Neither the United States government nor any agency thereof, nor any of their employees, makes any warranty, express or implied, or assumes any legal liability or responsibility for the accuracy, completeness, or usefulness of any information, apparatus, product, or process disclosed, or represents that its use would not infringe privately owned rights. Reference herein to any specific commercial product, process, or service by trade name, trademark, manufacturer, or otherwise does not necessarily constitute or imply its endorsement, recommendation, or favoring by the United States government or any agency thereof. The views and opinions of authors expressed herein do not necessarily state or reflect those of the United States government or any agency thereof.

Available to DOE and DOE contractors from:

Office of Scientific and Technical Information (OSTI)

P.O. Box 62

Oak Ridge, TN 37831

Prices available by calling (615) $576-8401$

Available to the public from:

National Technical Information Service (NTIS)

U.S. Department of Commerce

5285 Port Royal Road

Springfield, VA 22161

(703) $487-4650$ 


\section{DISCLAIMER}

Portions of this document may be illegible in electronic image products. Images are produced from the best available original document. 


\section{Table of Contents}

Background

Study Objectives

Task 1 - Landfill Cover Studies

Task 1.1 - Ash Collection and Mixture Preparation 5

Task 1.2 - Chemical Analysis of Ash Mixtures . 6

Task 1.3 - Microbiological Analysis 10

Task 1.4 - Botanical Growth Potential of Ash Mixtures 11

Task 1.5 - Physical and Structural Characteristics 11

Task 1 Summary of Findings 13

Task 2 - Evaluation of Ash as Aggregate for

Road Construction

Task 2.1 - Sieve Analysis

. Task 2.2 - Chemical Analysis of Screened Ash Fractions

Task 2.3 - Engineering Tests for Aggregate Use 18

Task 2 Summary of Findings 20

Conclusion

21

Acknowledgement

\section{Appendix A}

Appendix Al

Appendix A2

Appendix A3

Appendix A4

Appendix A5

Appendix A6

Appendix A7

Appendix A8

Appendix B

Appendix B1

Appendix B2

Appendix B3

Appendix B4

Appendix C

Appendix CI

Appendix C2
- Task 1

- Regulatory Requirements for Landfill Cover Materials

- Assh Collection, Storage, and Mixture Preparation

- Chemical Analysis of Ash Mixtures

- Enumeration of Fecal Coliform Densities in Ash/Sludge Mixtures

- Botanical Growth Potential of Ash Mixtures

- Erosion Resistance Test Results

- Permeability Test Results

- Ash TCLP Test Results

- Task 2

- Sieve Analysis Results

- Chemical Analysis of Screened Ash Eractions

- Permabase Preliminary Investigation

- Permabase Test Report

- Miscellaneous

- 1991 Mid-Connecticut Ash Test Results

- List of Abbreviations 


\section{EXECUTIVE SUMMARY}

This ash study investigates several aspects of Municipal Waste Combustion (MWC) ash utilization in order to develop an alternative to the present disposal practice of landfilling in a lined monofill.

Two major options were investigated. These were:

Task 1 - as a daily or final cover for municipal waste in the landfill.

Task 2 - as a road construction aggregate.

Statistically valid samples of eight mixtures of ash and other materials, and one' sample of soil were analyzed for chemical constituents. Biological tests on these mixtures were conducted, along with erosion tests and sieve analyses. In addition, a chemical analysis of each sieve size was conducted.

An engineering evaluation of the observable qualities of the sieve sizes was made, followed by a few engineering tests to measure geotechnical properties of the most promising materials. A test landfill cover section and a test road strip will be constructed in Phase II of the study.

Experts from the asphalt industry, state Road Division, Department of Health, and others who might properly have a role in implementation of any ash utilization program were involved from the start.

Significant findings to this point include:

1) Ali ash samples taken to date (over 400 samples over more than 4 years) have passed the EPA TCLP testing.

2) Chemical analysis of bottom and combined ash samples indicated less than expected variability.

3) Selected ash mixtures exhibited very low coefficients of hydraulic conductivity, less than $10^{-6} \mathrm{~cm} / \mathrm{sec}$ hydraulic conductivity.

4) All but one of the ash mixtures exhibited greater erosion resistance than the currently used landfill cover material. 
5) MWC combined ash chemical analysis, hydraulic conductivity, and erosion resistance indicates this ash fraction is a viable alternative for landfill cover.

6) MWC ash size fractions and chemical analysis show bottom and combined ash to be a viable alternative aggregate for road construction. Preliminary engineering test results show the H-POWER bottom and combined ash to be a promising potential product. 


\section{BACKGROUND}

\section{General}

The City \& County of Honolulu comprises the entire island of Oahu, where approximately 808 of the population 1800,000 plus a significant tourist population) of the state reside. To prevent possible contamination of the fresh water basal lens underlying most of the island, landfills must be sited around the periphery of the island. Since this is a prime area for tourists and residents, landfills are unpopular and difficult to site. It is therefore in the best interests of all to extend the current landfill life as long as possible. The City \& County of Honolulu has already made great strides toward this goal by incorporating a waste-to-energy facility (H-POWER) into their solid waste program. The City currently disposes of approximately 598,750 metric tons $(660,000$ tons) of municipal solid waste (MSW) annually at two sites, H-POWER and the Waipahu Incinerator. Over 908 of this annual waste stream is processed at H-POWER, where the volume is reduced to about 108 of the original MSW. The remaining ash, however, is currently being landfilled. Finding a beneficial use for the ash would mean that 908 of the island's solid waste stream is being completely recycled, and more importantly, being kept out of the landfill.

The H-POWER facility is a refuse derived fuel (RDF) plant with a nominal capacity of 544,320 metric tons $(600,000$ tons) of MSW per year, and generates a maximum output of $57 \mathrm{MW}$ of electricity. The RDF processing facility is equipped with a ferrous metal separation system. The power block facility employs two RDF-fed boilers, each equipped with a dry scrubber and a five field electrostatic precipitator (ESP). The bottom ash is processed through a vibrating finger screen, ferrous magnet, and an eddy current separator where both ferrous and non-ferrous metals are separated from the ash. Fly ash is removed from each ESP field and from the dry scrubber cyclone tower as a dry powder, and run through a pug mill where water is added to reduce the dust. This fly ash stream is then combined with the bottom ash, placed in a trailer, and hauled to the landfill. Approximately 108,864 metric tons $(120,000$ tons) of wet ash is produced annually composed of approximately 608 bottom and 408 fly ash.

Also included in this study is ash from the Waipahu Incinerator. This is a two unit, 544 metric ton/day 1600 ton/day) mass-burn facility located in Waipahu, Hawaii. Each unit has a water spray and a three field ESP to control 
ton/dayl mass-burn facility located in Waipahu, Hawaii. Each unit has a water spray and a three field ESP to control

particulate emissions. This incinerator does not employ any energy recovery and was included in the study so that results could be applied to mass-burn as well as RDF facilities. The Waipahu Incinerator currently operates at approximately 181 metric tons per day (200 tons per day) [approximately 54,432 metric tons/year $(60,000$ tons/year $)]$ 
The objective of the study was to develop low-cost options for utilizing MWC ash in a productive and environmentally responsible manner. The options investigated were divided into two tasks:

Task 1 - using MWC ash as landfill cover -- as
daily and/or final-closure cover.

Task 2 - using MWC ash as a road construction aggregate.

\section{TASK 1}

Task 1 was designed to investigate the feasibility of using various MWC ash mixtures as daily or 'final-closure cover. Appendix Al outlines the regulatory requirements for materials used as landfill cover. Figure 1 below lists the mixtures used. Note. that mixture 4 is the soil material currently used as a daily cover material.

ASH MIXTURES

\begin{tabular}{|c|l|}
\hline Mixture & \multicolumn{1}{|c|}{ Mixture Contents } \\
\hline 1 & H-POWER Fly Ash \\
\hline 2 & H-POWER Bottom Ash \\
\hline 3 & H-POWER Combined Ash \\
\hline 4 & Landfill Soil (control) \\
\hline 5 & $\begin{array}{l}\text { 3 Parts H-POWER Fly Ash to 1 Part } \\
\text { Sewage Sludge }\end{array}$ \\
\hline 6 & $\begin{array}{l}\text { 1 Part H-POWER Fly Ash, 1 Part AES } \\
\text { Coal Ash, 1 Part Sewage Sludge }\end{array}$ \\
\hline 7 & $\begin{array}{l}\text { 1 Part H-POWER Fly Ash to 1 Part } \\
\text { Sewage Sludge }\end{array}$ \\
\hline 8 & $\begin{array}{l}2 \text { Parts H-POWER Fly Ash, 2 Parts } \\
\text { Sewage Sludge, 1 Part Hydrated Lime }\end{array}$ \\
\hline 9 & $\begin{array}{l}1 \text { Part H-POWER Combined Ash to 1 } \\
\text { Part Waipahu Ash }\end{array}$ \\
\hline
\end{tabular}

$$
\text { Figure } 1
$$

Mixtures including sterile sludge from the City's wastewater treatment plant were also tried in order to ascertain the viability of mixing this material with ash. It was 
anticipated that mixing this ash with sludge would preclude or reduce any odors, and perhaps result in a soil-like material more suitable for plant growth.

Task 1 was divided into several subtasks. All mixtures were subjected to chemical analysis and microbiological testing. General physical and structural characteristics were observed in addition to placing each sample in a test erosion box where it was subjected to natural weathering and erosion.

Mixtures $4,5,6,7$, and 8 were provided to the botanical garden operated by the City Parks Department for testing of their potential to support growth of several common grasses and weeds.

\section{TASK 2}

Hawaii currently employs volcanic rock for it's aggregate use. Communications with representatives from Grace Pacific (one of two major aggregate. suppliers) and with a purchaser of aggregate on Oahu indicated that aggregate smaller than the \# 8 sieve size are in the greatest demand. This is because these smaller sizes are costlier to produce as they require more crushing and separation steps. All aggregate supply representatives consulted agreed that an alternative aggregate source would be a welcome addition to the market. Task 2 was designed to investigate some of the numerous possible applications for MWC ash as road construction material. This investigation involved sieve analysis, chemical analysis, and mechanical testing.

Task 2 was also divided into several subtasks. The first of these was a sieve analysis, followed by chemical analysis of each sieve size. A qualitative review of the various sieve fractions was conducted by personnel knowledgeable in the asphalt road construction business, and based on their recommendations, dry density tests, unconfined compressive strength tests, sand equivalent tests, plasticity index measurements, and LA Abrasion testing were conducted. In addition, samples of the ash material were provided to Permabase, Inc. for analysis and testing in their products, as well as providing an opportunity to compare our material with that from MWC ash in Florida. The objective here was to evaluate the potential for use as a soil cement component, an application which has been tested in Florida, New Hampshire, Minnesota, and several European Countries. 
Task 1 - Landfill Cover Studies

Phase I study conducted certain chemical and physical tests on each of the mixtures shown in Figure 1. Observations were also made over a 4-month period on inclined erosion test boxes containing each of the test mixtures. These observations were made to see the effects of rain and wind erosion, to observe how easily the mixture would compact, what their lateral shear strengths were, and how easily the surface cracked after drying.

Task 1.1 - Ash Collection and Mixture Preparation

\section{Ash Collection}

The MWC ash used in the study was collected in small increments over a five day period to insure representative sampling. The date, time, quantity and storage drum destination was noted and recorded for each sample (Appendix A2 Tables 1 - 6). The 209-liter (55-gallon) steel storage drums were obtained from a local dairy and were in excellent condition. The drums were prepared by lining them with a 4-mil fitted, plastic drum liner. Once filled, the liner was sealed to keep the moisture level relatively constant and to prevent possible contamination.

Processed bottom ash samples were taken directly from the ash loadout trailer located beneath the bottom ash metal recovery unit (BAMR). Dry fly ash was collected from a 10-centimeter (4-inch) steel pipe mounted on the side of the surge bin, prior to the ash reaching the pug mill. Photographs and a description of the H-POWER ash loadout procedures are included in Appendix A2, pages 1-3.

Waipahu Incinerator ash used herein was collected at the ash monofill located directly across from the incinerator (Appendix A2, figure 3). All of the Waipahu ash was first run through BAMR at H-POWER, however, and the processed ash was then loaded into steel drums for storage. Figure 2 below shows a comparison of the Waipahu ash and H-POWER bottom ash after processing through the BAMR. 
Bottom Ash Metal Recovery Operation

\begin{tabular}{|l|c|c||}
\hline Separation Stream & H-POWER Bottom Ash & $\begin{array}{l}\text { Waipahu Incinerator } \\
\text { Ash }\end{array}$ \\
\hline Screened Ash & $66 \%$ & $72 \%$ \\
\hline Non-Ferrous Metals & $2 \%$ & $17 \%$ \\
\hline Ferrous Metals & $12 \%$ & $10 \%$ \\
\hline Other & $20 \%$ & $1 \%$ \\
\hline
\end{tabular}

Eigure 2

\section{Mixture Preparation}

Preparation of the nine test mixtures was accomplished using a gas-powered cement mixer. Water was added in small amounts until a clumpy, semi-dry paste was formed with a plasticity high enough that the mixture could be easily spread out, but not such that it couldn't hold its shape. The details of mixture preparation are included in Appendix A2, paige 10.

\section{Task 1.2 - Chemical Analysis of Ash Mixtures}

\section{Sampling Methods}

After the Task 1 mixtures had cured for a period of no less than 72 hours, they were individually dumped out, mixed using a hand trowel, and quartered. Duplicate samples of each mixture were taken randomly from each of the four quarters, placed in a $500 \mathrm{ml}$ plastic sample bottle, labeled, and transported to Environmental Laboratory of the Pacific (E.L. Pacific).

E.L. Pacific performed acid digestion using a combination of nitric, perchloric and hydrofluoric acids to dissolve all of the solids. The solutions were then tested for the following metals content: $\mathrm{Al}, \mathrm{As}, \mathrm{Ba}, \mathrm{C}, \mathrm{Cd}, \mathrm{Ca}, \mathrm{Cr}, \mathrm{Cu}, \mathrm{Fe}, \mathrm{Pb}, \mathrm{Hg}, \mathrm{K}$, $\mathrm{Se}, \mathrm{Si}, \mathrm{Ag}$, and $\mathrm{Zn}$. Other chemical analyses included $\mathrm{pH}, \% \mathrm{Cl}$, percent soluble $\mathrm{SO}_{4}, \mathrm{NO}_{3}, \mathrm{NO}_{4}$, total nitrogen, and moisture content. Titration curves were also prepared for duplicate samples of the 9 mixtures. The EPA analysis methods used are listed by each chemical parameter in Appendix A3, page 1. 
A detailed discussion of the results of the Task 1 chemical analyses is provided along with the data in Appendix A3, pages 1-12. The entire E.L. Pacific laboratory report can be found following the analysis (page A3-13). Figure 3 below provides a summary of the average concentrations of trace metals for the ash mixture major constituents.

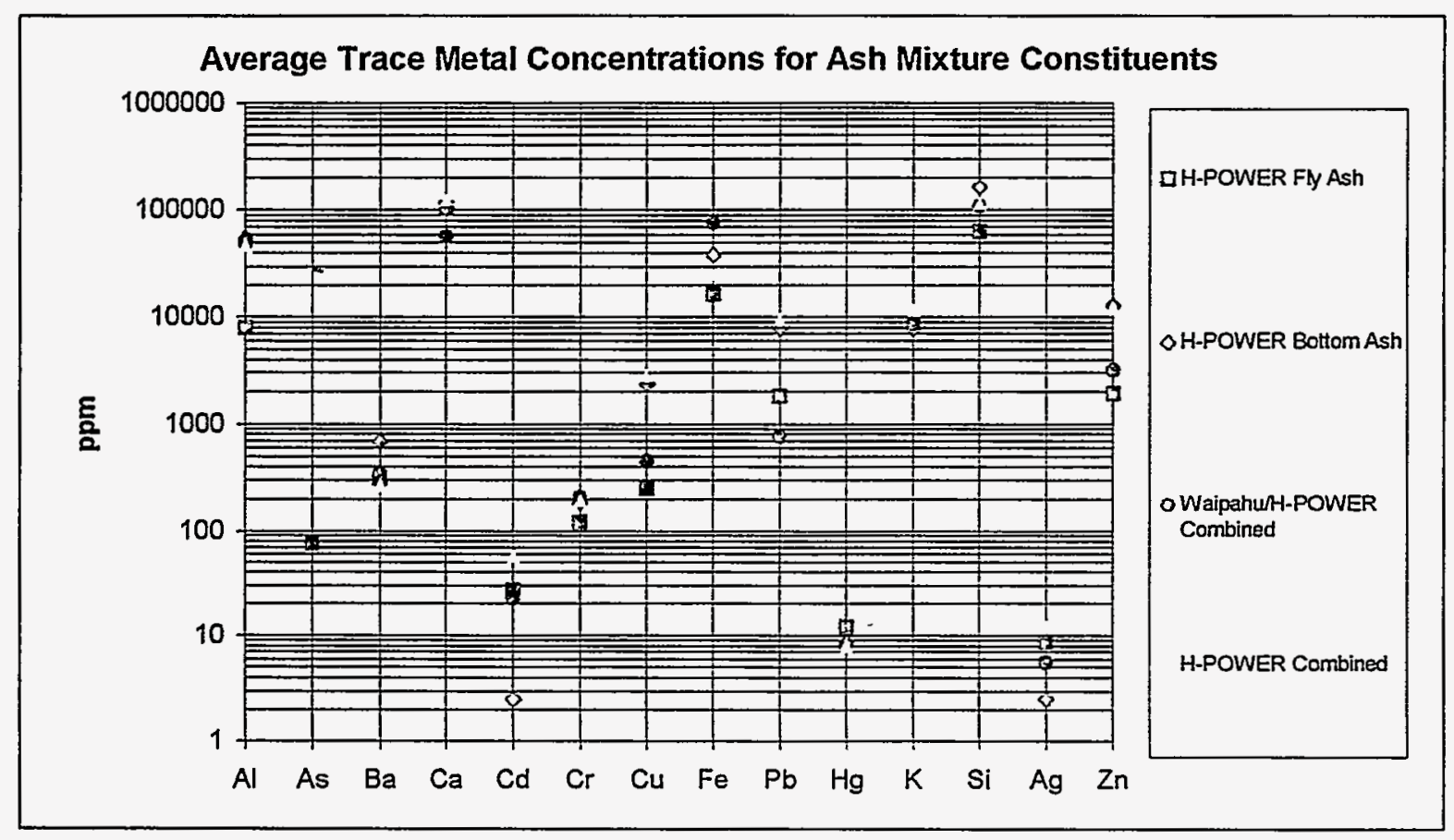

Figure 3

Bear in mind that these concentrations are not leachate concentrations and should not be interpreted as such.

H-POWER Ash TCLP data can be found in Appendix A8.

A few notable trends in the trace metal analysis are as follows:

1. Cadmium is only about 8 times higher in the fiy ash than the bottom ash.

2. Mercury is in relatively low concentration in all ash fractions and uniformly distributed. 
3. Mercury is in relatively low concentration in all ash fractions and uniformly distributed.

4. Metal concentrations are within 1 order of magnitude from one ash type to another.

In most cases the variation in chemical characteristics

between the ash mixtures and the soil controls were within 1 order of magnitude of each other. The elements or chemical parameters where the ash mixtures were significantly higher than the control concentrations are illustrated in figures 4 through 6 below:

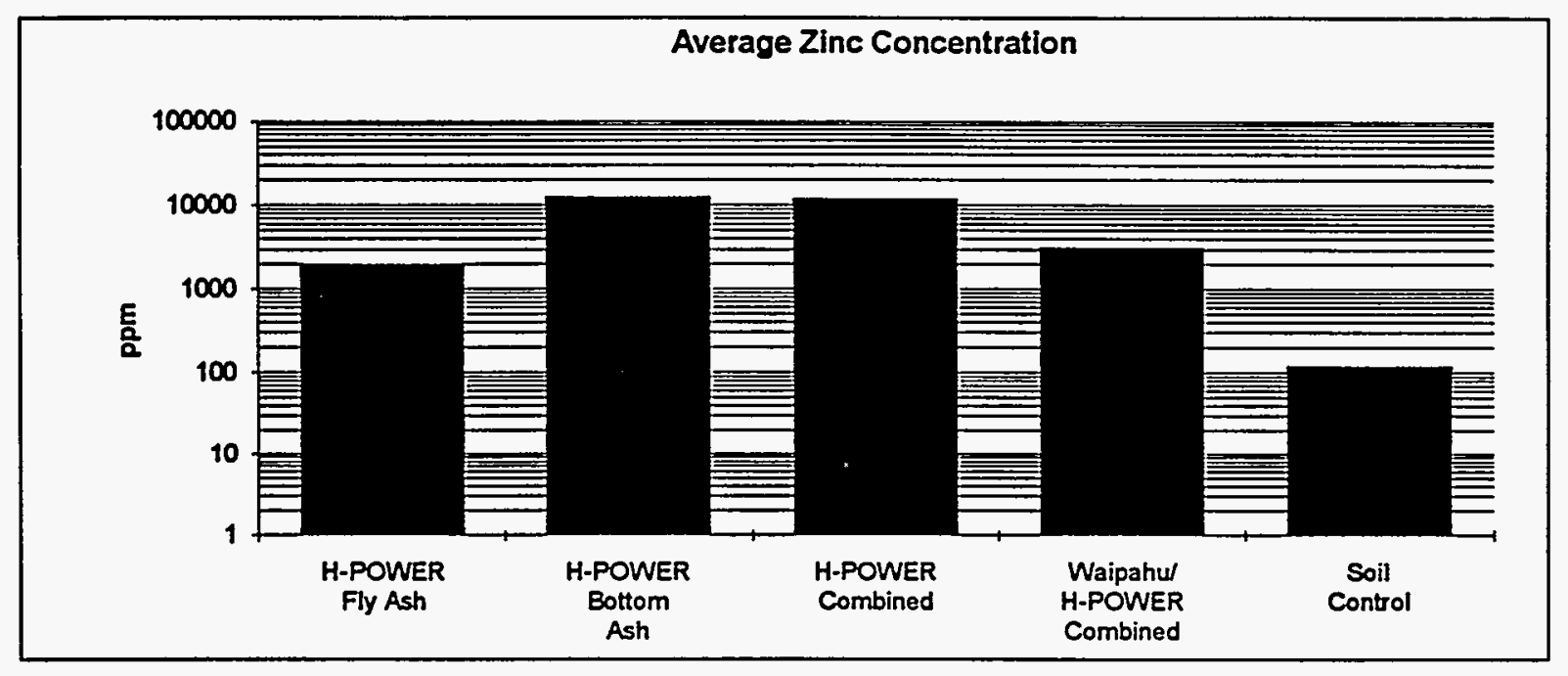

Figure 4

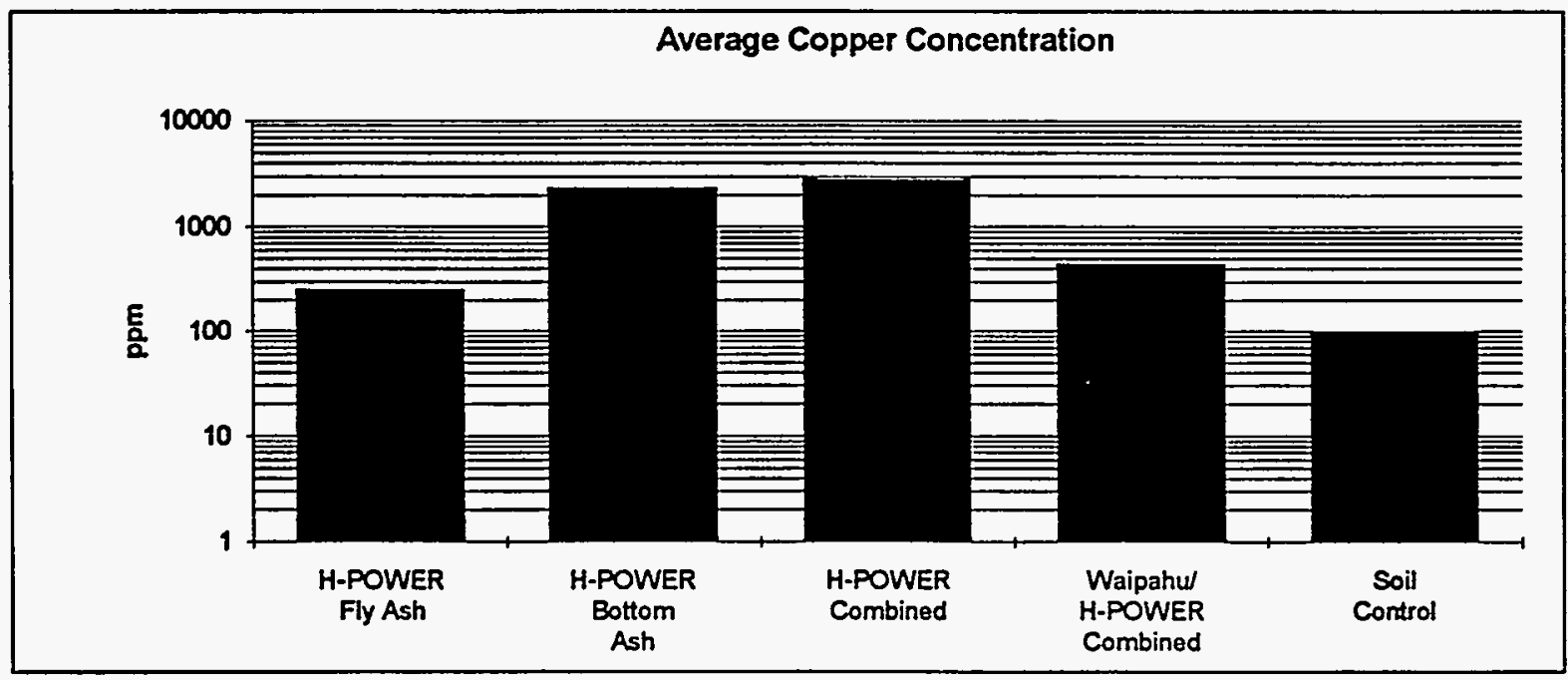

Figure 5 


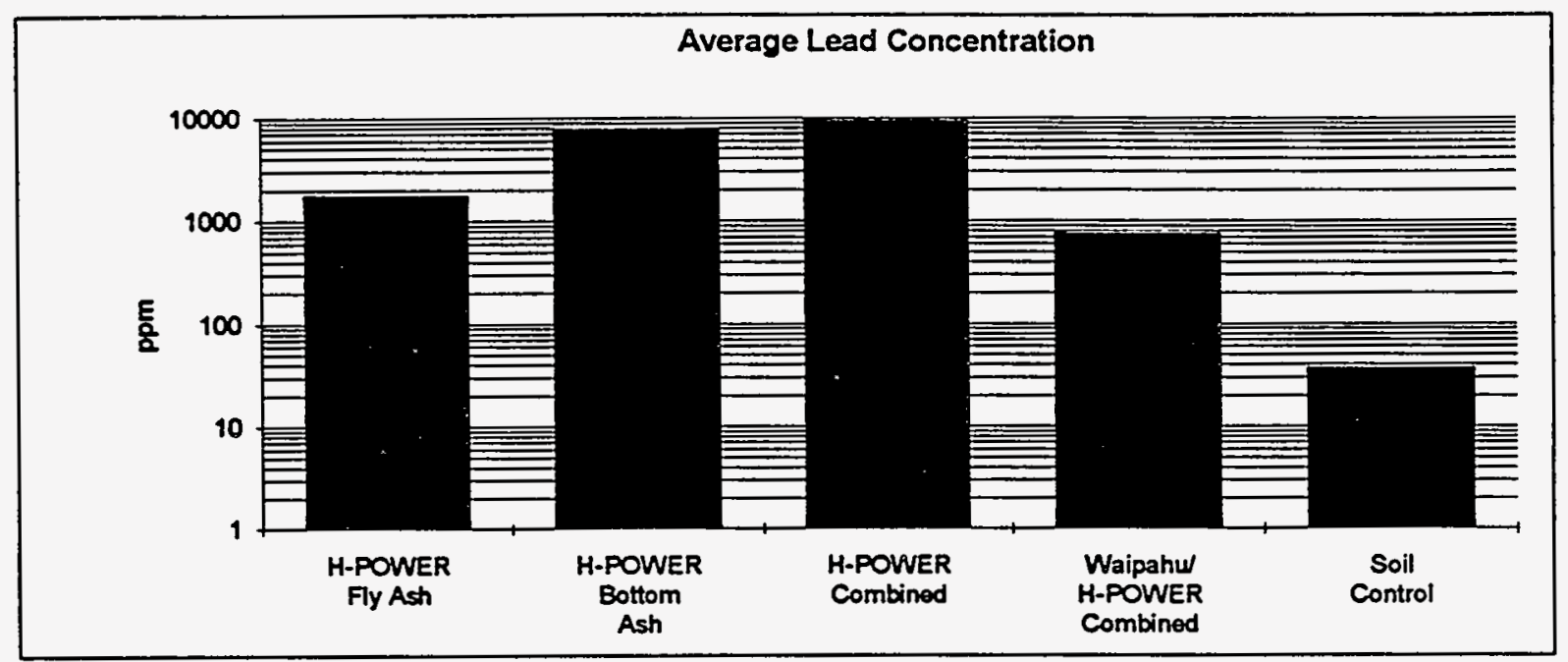

Eigure 6

One other notable difference was that the $\mathrm{pH}$ levels in all ash mixtures averaged around 11.8 compared to 8 for the soil control.

\section{Acid Neutralization Curves}

Acid neutralization (titration) curves were run on each duplicate mixture sample. The titrations were carried out by combining 10 grams of each mixture sample with $50 \mathrm{ml}$ of distilled water. While the resulting solutions were constantly being stirred, they were titrated with $1 \mathrm{~N}_{2} \mathrm{SO}_{4}$ and 1 minute $\mathrm{pH}$ readings were recorded. The resulting curves indicate, among other things, the buffering capacity of the various mixtures and are shown in Appendix A3, beginning on page 22 .

The analysis of the data presented was accomplished by identifying the $\mathrm{pH}$ level corresponding to the inflection points in the curve. In general, $\mathrm{pH} 4.5$ marks the point at which all of the carbonates and bicarbonates have been converted to carbonic acid. Therefore, the amount of acid required to reach this point can be reported as the total alkalinity of the ash mixture solution. The following figure presents the alkalinity of each mixture as $\mathrm{g} / \mathrm{L} \mathrm{CaCO}_{3}$. 


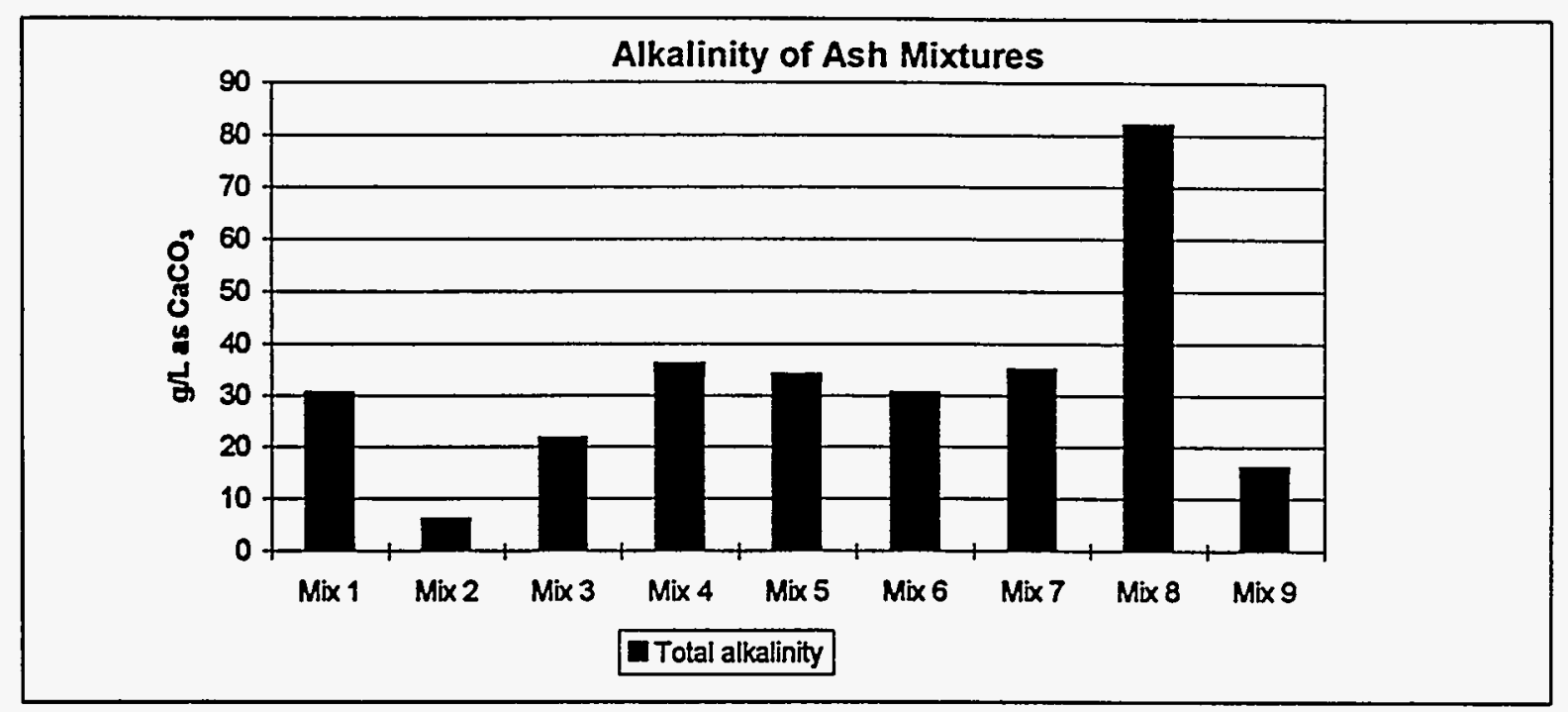

Figure 7

As the data in Figure 7 demonstrate, with only two exceptions, the total alkalinity and consequently the buffering capacity of the mixtures is relatively constant compared to the soil control (mixture 4). The extreme outliars are mixture 2 at 6 $\mathrm{g} / \mathrm{L}$, and mixture 8 at $82 \mathrm{~g} / \mathrm{L}$. This comparatively high alkalinity is a result of the additional lime added in preparation of this mixture.

\section{Chemical Analysis Findings}

Overall, there appears to be nothing from this chemical data to indicate ash mixtures would cause endangerment of human health or the environment when used as an alternative to natural soil as daily landfill cover.

\section{Task 1.3 - Microbiological Analysis}

The initial objective of the microbiological analysis was to ascertain the potential of MWC ash to reduce unpleasant odors and neutralize fecal coliform bacteria in sewage sludge. Tests were also conducted to determine the existence of Salmonella in mixtures containing sewage sludge. Salmonella test results appear on page 4 of Appendix A4, following the fecal coliform report. It was subsequently determined that the sludge from the City's wastewater treatment facility is sterile when it leaves the facility, and this was confirmed by 
the negative findings of the microbiological analysis. See Appendix A4 for lab findings.

\section{Task 1.4 - Botanical Growth Potential of Ash Mixtures}

Five MWC ash mixtures $(4,5,6,7$, and 8 ) were tested to ascertain their potential to support botanical growth by attempting to grow grass. Appendix A5 contains a description of the test procedures and findings.

Botanical Findings Summary

After 10 weeks, all of the grass samples had died. Root growth was not substantial and it is believed that the test grass samples were able to survive for so long by living off top soil clinging to the roots prior to transplanting. Germination of the seeds was poor in all cases except for the Australian Carpet Grass which germinated better in some ash mixtures than in the botanical garden's control soil. The seedlings were also dead within 10 weeks. In both cases, the ash mixtures could not sustain long-term plant growth because of their hard, cement-like characteristics which provided insufficient water drainage and oxygen. It is likely that the low nitrogen content and high pH level were also major factors in the growth inhibiting properties of the mixtures. In Phase II, further studies are proposed to evaluate mixing combined ash with soil in a $1: 4$ ratio and adding nitrogen rich fertilizer.

Task 1.5 - Physical and Structural Characteristics

Erosion Resistance Test

The objective of the erosion test was to determine the MWC ash mixtures' ability to resist wind and rain erosion. Mixtures 1 through 9, (Figure 1) were placed to a depth of approximately two inches in a wooden box of approximately 1 square meter (12 sq. ft.), mounted at a $15^{\circ}$ angle. The face of each mixture was leveled off as smoothly as possible. In the two months to follow, photographs were taken every ten days and visual observations were recorded as necessary. Some of the photographs taken are included in Appendix $A 6$ along with a description of the findings.

At the end of the test period, each of the mixtures were examined and ranked according to the following pass/fail criteria: erosion resistance, material strength, and 
shrinkage. Figure 8 below shows the qualitative evaluation of the various mixtures.

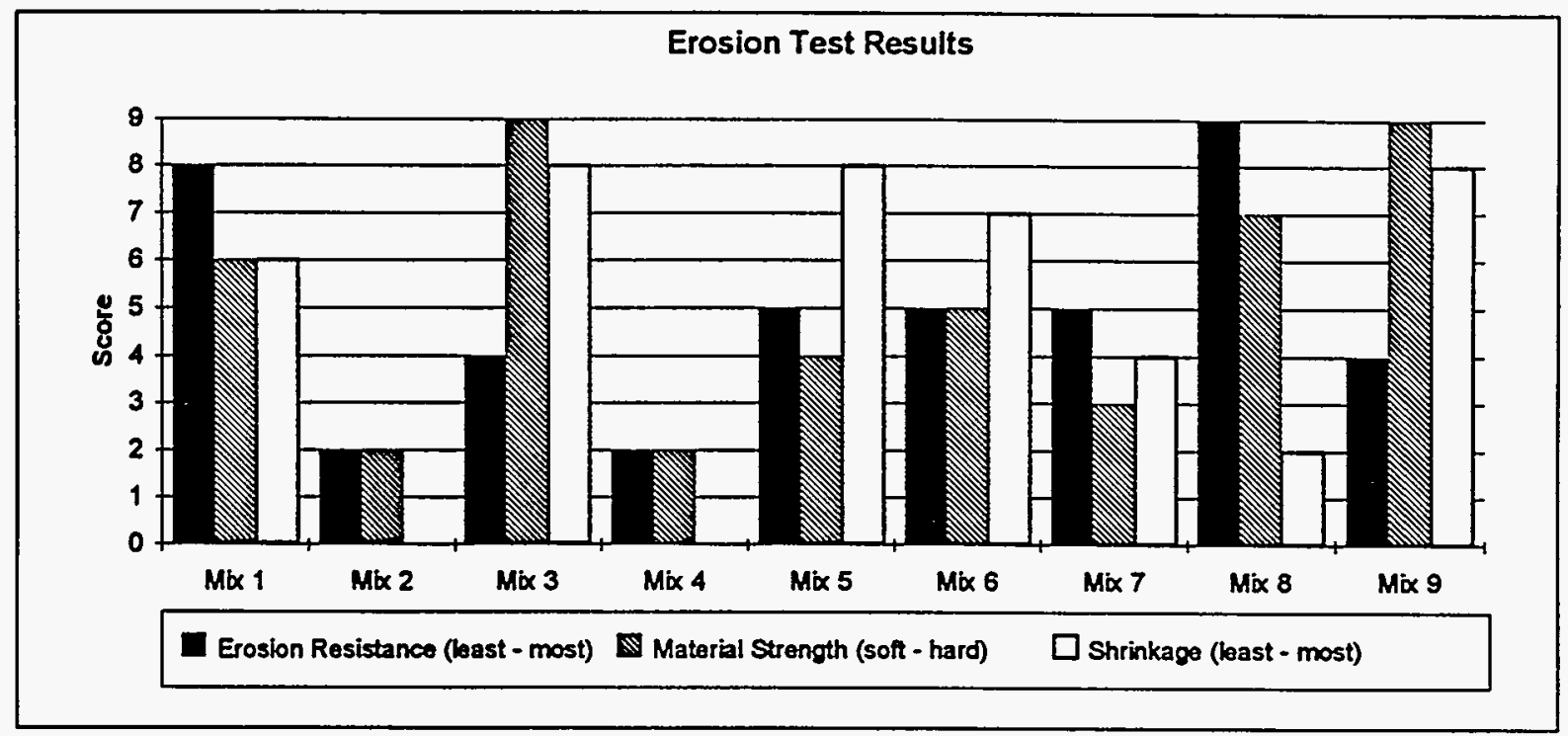

Eigure 8

Note that mixture \#8 was the most resistant to erosion while \#2 and \#4 were the least resistive as expected by their loose nature. With the exception of mixtures \#2 and \#4, the overall performance of the ash mixtures was essentially similar, with no single mixture clearly out-performing the others. As a result, the most economical mixture was chosen as the top performer for this subtask. Mixture \#3 is clearly the most economical in that it represents the entire ash stream produced by H-POWER, requires no additional processing, no stabilizing additives, and no mixing.

\section{Permeability Test}

These tests were included in the protocol to evaluate the various mixtures with respect to permeability characteristics. The objective was to determine whether any of them could meet the Hawaii subsection 58.1-17 requirements (outlined in Appendix Al) for final landfill cover. The analysis utilizes a falling head permeameter and to insure accurate results, three specimens from each sample were subjected to a minimum of four runs each.

Laboratory findings indicate that the mixtures tested possess very low coefficients of hydraulic conductivity meeting the permeability requirements for the infiltration inhibiting layer (no greater than $10^{-5} \mathrm{~cm} / \mathrm{s}$ ). The results of the 
permeability testing along with a description of the findings are included in Appendix A7.

\section{Task I Conclusions from Findings}

The Task 1 test results indicate that MWC ash mixtures pose no apparent threat to human health or the environment. This will be further elaborated on in Phase II with the conduct of a health risk evaluation. Most of the ash mixtures have superior infiltration and erosion resistance material at the same relative buffering capacity as the presently used cover material. Although most of the ash mixtures would be deserving of large scale field testing, the decision to narrow the field down to one or two mixtures was made in the early planning stages so as to focus more intensely on each mixture. As a result, it has been decided to carry mixture \#3 (H-POWER combined ash) into the second phase of the project. 
TASK 2 -Evaluation of Ash as Aggregate for Road Construction

Task 2 efforts involved conducting sieve analyses on the three MWC ash types (H-POWER bottom, combined, and Waipahu combined ash) and subsequent chemical and physical analyses of the individual size fractions. Based on a qualitative review by professionals in the paving industry, certain fractions of the three MWC ash types were selected for further engineering properties testing to determine the suitability of the ash as a road construction aggregate.

\section{Task 2.1 - Sieve Analysis}

Samples of each ash type were oven-dried in preparation for sieving. After drying, the samples were analyzed using the following U.S. Standard sieve sizes: $3 / 4 "$ (19.05 mm), 1/2" $(12.7 \mathrm{~mm}), 3 / 8 "(9.53 \mathrm{~mm}), \# 4(4.75 \mathrm{~mm}), \# 8(2.36 \mathrm{~mm}), \# 16$ $(1.18 \mathrm{~mm}), \# 30(0.60 \mathrm{~mm}), \# 50(0.30 \mathrm{~mm}), \# 100(0.15 \mathrm{~mm})$, and \#200 (0.075 mm). Figure 9 below shows a summary of the sieve analysis for each ash type:

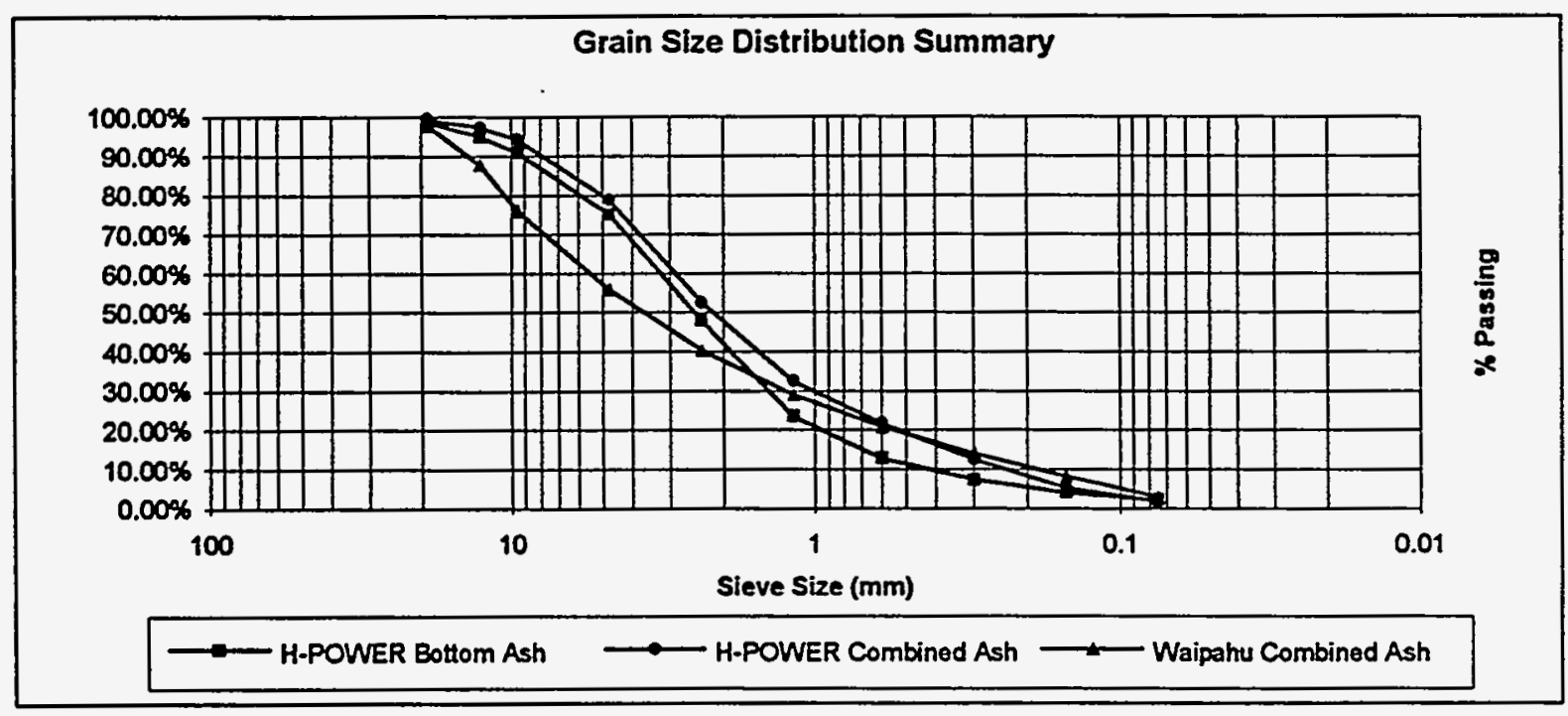

Figure 9

Results of the individual sieve analyses and an explanation of the data are found in Appendix Bl.

A qualitative review of the size fractions found the H-POWER combined ash to be the most promising material. Although it was desirable to use all the material passing the \#4 or 3/8" sieve, it turned out that the material larger than the \#16 sieve size was simply unfit for use in asphalt as there were 
occasional pieces of metal wire and long flat pieces of ceramic and glass. Fortunately, the minus \#16 material still accounts for over $50 \%$ of the H-POWER combined ash gradation.

One interesting point to note is that while a sieve analysis conducted on the fly ash alone indicated approximately 508 passing the \#200 sieve, the gradations of the H-POWER combined ash did not reflect the same characteristic (compare Figures 2 and 5, [page 8 and 23] of Appendix B1). This anomaly is believed to be a result of pelletizing of the fly ash as it was mixed with the wet bottom ash. It is important to note in. this case that the gradations of combined ash samples which were mixed in a dry state provide considerably more fine material than those mixed wet. Figure 10 below illustrates this phenomenon.

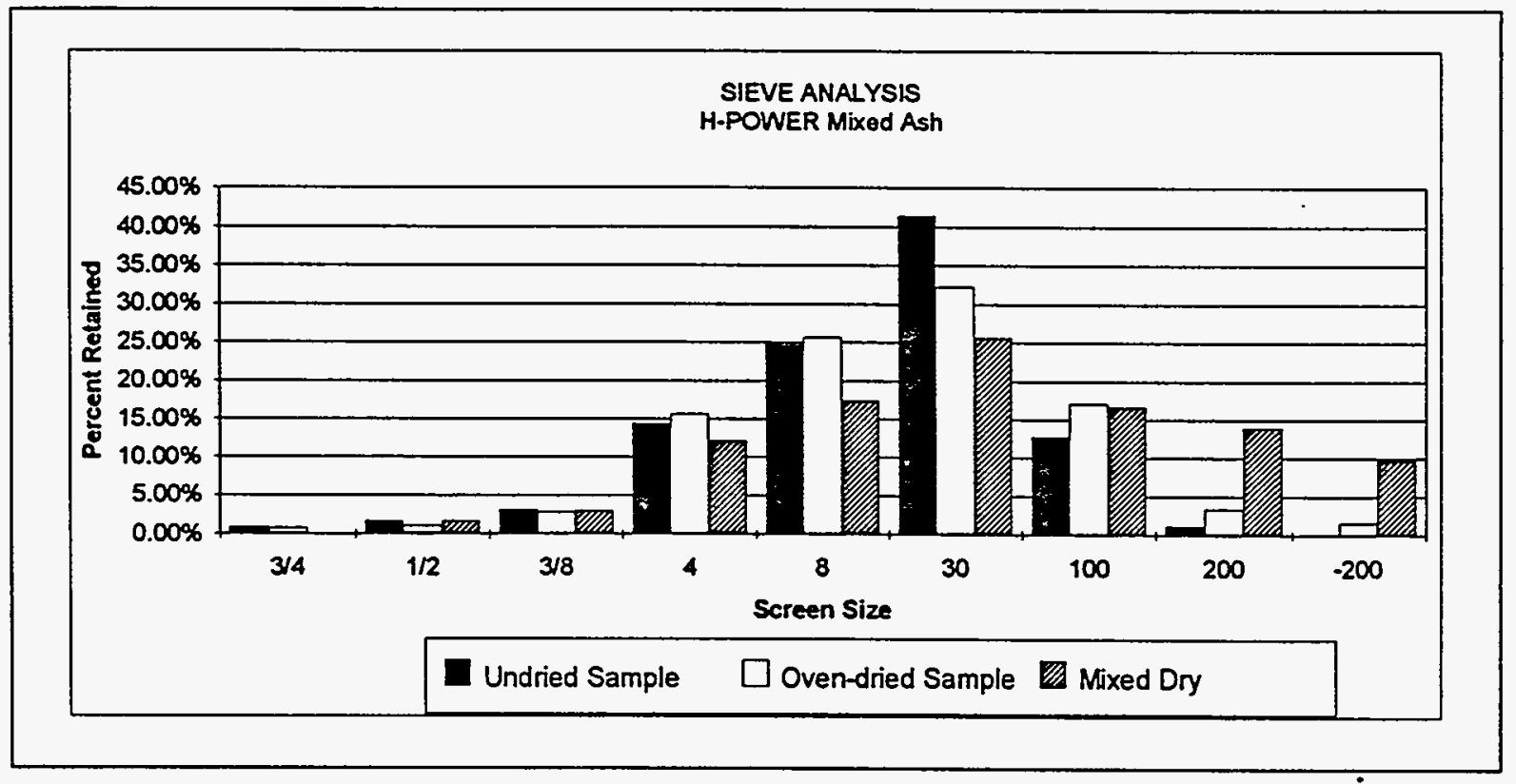

Figure 10

Task 2.2 - Chemical Analysis of Screened Ash Fractions

Sampling and Analysis Methods

Representative samples (about $5 \mathrm{ml}$ each) were taken from each of the 8 size fractions from each of the three types of ash (H-POWER combined ash, H-POWER bottom ash, and Waipahu combined ash). Individual chemical analyses were carried out on each sample for: $\mathrm{Al}, \mathrm{Cd}, \mathrm{Ca}, \mathrm{Cu}, \mathrm{Fe}, \mathrm{Na}, \mathrm{Pb}, \mathrm{Zn}, \mathrm{Hg}, \mathrm{Si}$, 
$\%$ chlorides, \% sulfates, $\mathrm{pH}, \%$ moisture, and density. The analytical chemistry methods used for these analyses are listed by each chemical parameter in Appendix B2.

\section{Chemical Findings Summary}

A detailed discussion of the results of the Task 2 chemical analyses is provided along with the data in Appendix B2. The entire E.L. Pacific laboratory report can be found following the analysis. Figures 11, 12, and 13 below present a summary of the trace metal concentrations for the three different ash types by sieve size. The sieve sizes indicated represent the material retained on that particular screen size.

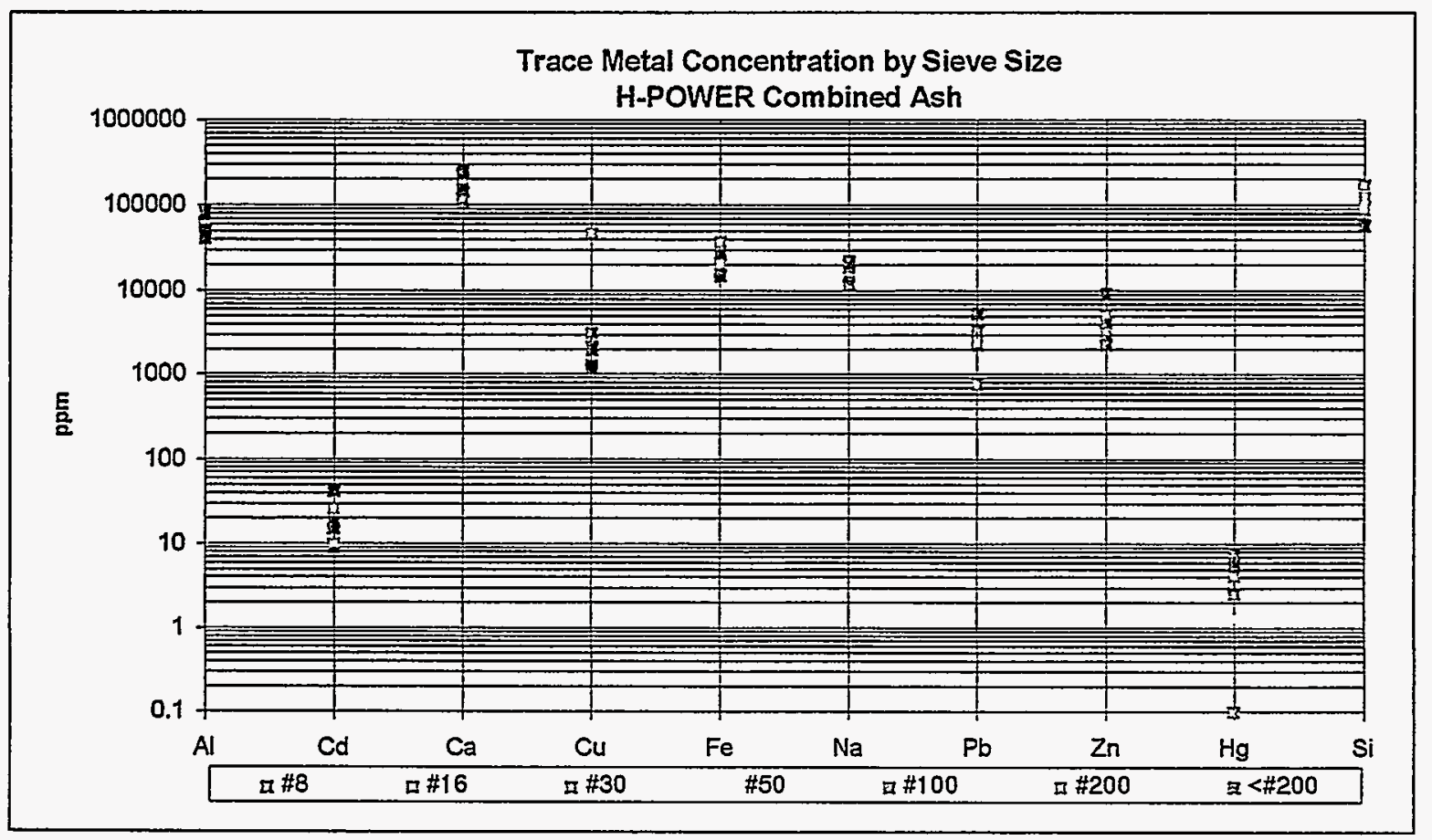

Figure 11 


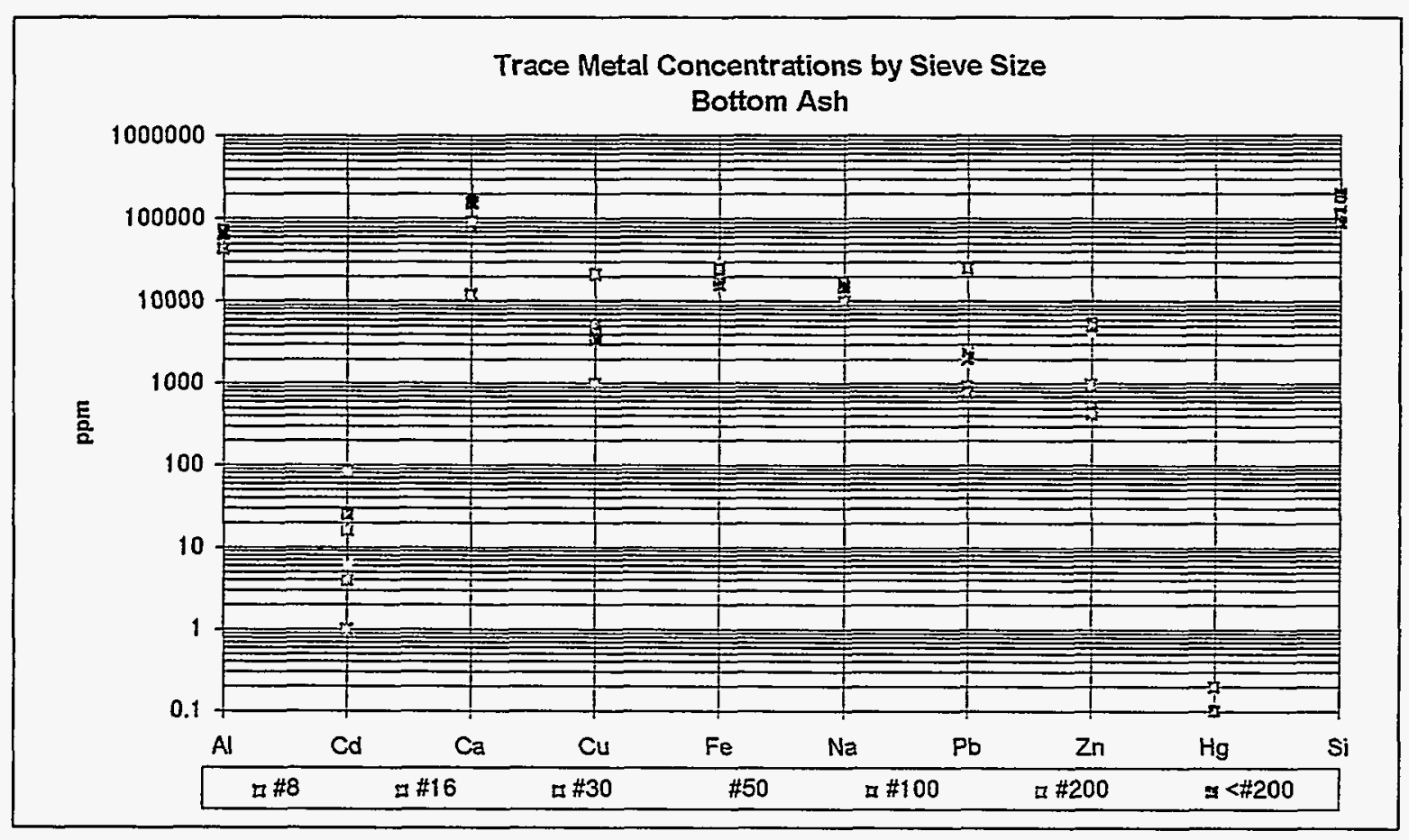

Figure 12

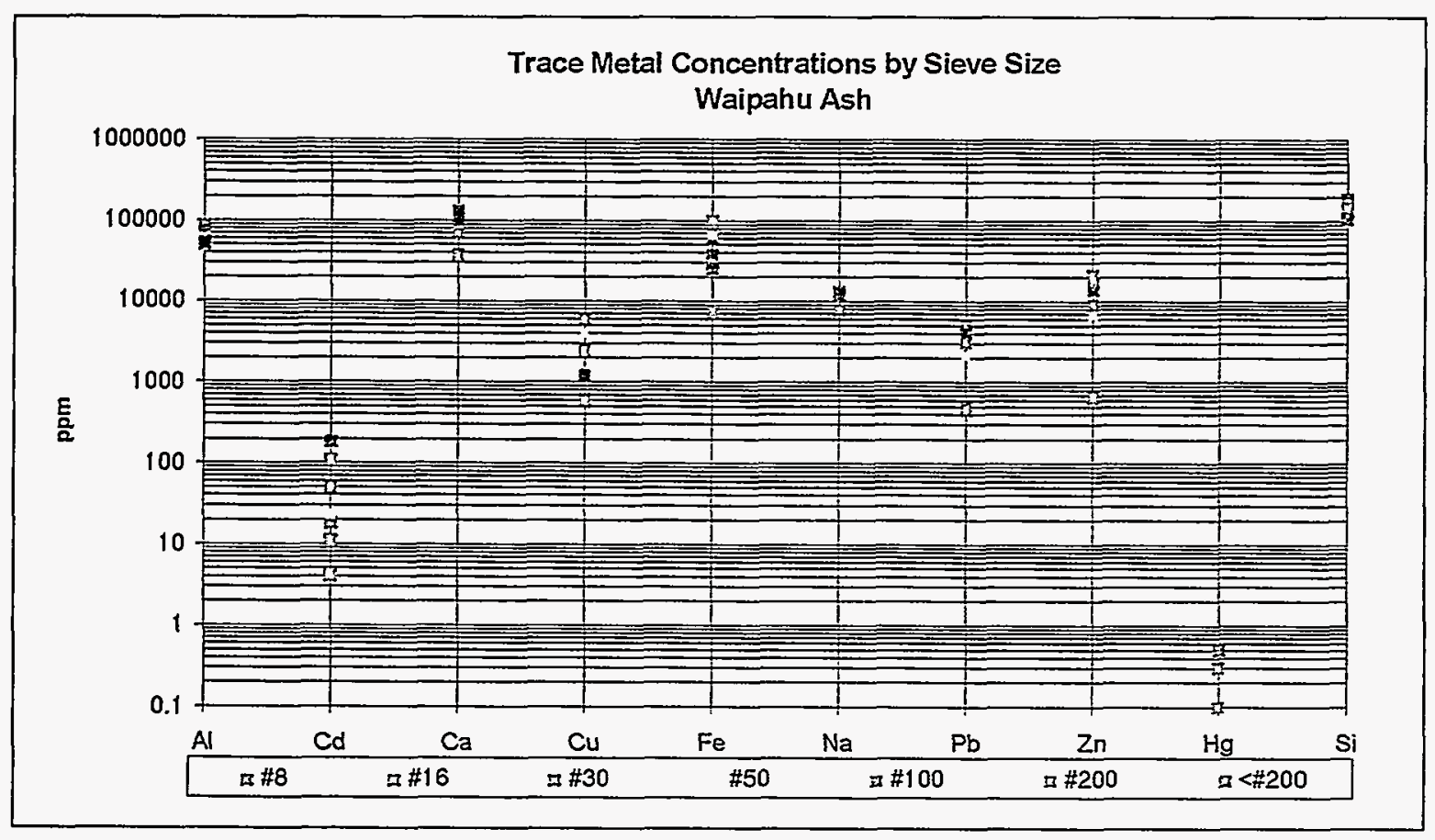

Figure 13 
It is important to note that the three ash types were reasonably consistent in metal concentrations among the seven different size fractions.

Traditionally, the municipal waste combustion ash most often utilized as aggregate in road construction is bottom ash. These data show combined ash to be chemically equivalent to bottom ash, and, thus, equally relevant for aggregate use. With some modification to its BAMR unit, H-POWER could produce a screened fraction of this ash starting at sieve size \#16. This could result in potential utilization of $50 \%$ of this ash fraction.

\section{Task 2.3 - Engineering Tests for Aggregate Use}

During the early stages of Phase I, a meeting with representatives of the two local paving companies and Florida-based Permabase, Inc. was held to discuss possible applications of H-POWER bottom ash as a substitute road construction aggregate. Permabase, Inc. is a roadbase material supplier interested in using H-POWER ash in their patented soil cement mixture, PERMABASE-PLUS. Permabase conducted a preliminary investigation using H-POWER ash in their soil cement product and achieved seven-day strengths which were "well above the typical strengths achieved with sand mixtures" (see Appendix B3, page 3). These positive preliminary findings moved Permabase to conduct a more thorough investigation involving H-POWER ash. Several laboratory tests were carried out on H-POWER ash and locally available Hawaiian construction materials. The purpose of these tests was to determine the suitability of H-POWER ash for use in PERMABASE-PLUS while meeting Hawaii Department of Transportation (HDOT) specifications. The complete Permabase Task 2 investigation report is found in Appendix B4.

Analysis Methods and Sampling

PERMABASE-PLUS is a combination of MWC ash, cement, water and locally produced aggregates. Each of these ingredients can be utilized in varying amounts depending on the quality and availability of the raw materials, and the structural and environmental specifications which apply. According to the Hawaii Department of Transportation's (HDOT) Standard Specifications for Road and Bridge Construction - Section 308, soil cement is locally known as Portland Cement Treated Base and is required to be tested by the following procedures: 
soil cement is locally known as Portland Cement Treated Base and is required to be tested by the following procedures:

$\begin{array}{llll}\text { Los Angeles Abrasion } & \text { AASHTO T } 96 \\ \text { Sand Equivalent } & \text { AASHTO T } 176 \\ \text { Plasticity } & \text { AASHTO T } 90 \\ \text { Flat or Elongated Pieces } & \text { HWY-TC } 4 & \\ \text { Grading } & \text { AASHTO T } 27\end{array}$

H-POWER bottom and combined ash were used for these tests along with local Red Base Rock, \#4 Rock, and \#3 Sand; the latter three supplied by Grace Pacific Corporation (GP) from their rock quarry and processing facility on Oahu. After evaluation of the five materials received and a review of the specifications, Permabase, Inc. decided to utilize GP \#4 Rock and GP \#3 Sand in combination with H-POWER combined ash.

Due to the sample size and time limitations, Permabase, Inc. prepared four aggregate combinations using the following criteria: a minimum content of $25 \%$ ash as a practical level of recycling necessary for commercial viability, and a maximum of $50 \%$ ash due to HDOT specification limits. Note that if the aggregate mixtures are found to have commercial applications, the optimum aggregate combination can be determined through practice and experience. The four aggregates prepared for testing are in the concentrations shown in Figure 15:

\begin{tabular}{|c|c|c|c|c|c|}
\hline $\begin{array}{l}\text { Aggregate } \\
\text { Mix }\end{array}$ & $\begin{array}{l}\text { H-POWER } \\
\text { Combined } \\
\text { Ash }\end{array}$ & $\begin{array}{l}\text { GP \# 4 } \\
\text { Rock }\end{array}$ & $\begin{array}{l}\text { GP \#3 } \\
\text { Sand }\end{array}$ & $\begin{array}{l}\text { Crushed } \\
\text { Concrete- } \\
\text { Coarse }\end{array}$ & $\begin{array}{l}\text { Crushed } \\
\text { Concrete- } \\
\text { Fine }\end{array}$ \\
\hline 1 & 258 & 508 & 258 & $\mathrm{n} / \mathrm{a}$ & $\mathrm{n} / \mathrm{a}$ \\
\hline 2 & 508 & 508 & $\mathrm{n} / \mathrm{a}$ & $\mathrm{n} / \mathrm{a}$ & $\mathrm{n} / \mathrm{a}$ \\
\hline 3 & 258 & $\mathrm{n} / \mathrm{a}$ & $-\mathrm{n} / \mathrm{a}$ & 508 & 258 \\
\hline 4 & 508 & $\mathrm{n} / \mathrm{a}$ & $\mathrm{n} / \mathrm{a}$ & 508 & $\mathrm{n} / \mathrm{a}$ \\
\hline
\end{tabular}

Eigure 15

NOTE: $\quad$ Coarse graded aggregates are larger than the \#4 $(4.75 \mathrm{~mm})$ sieve.

Aggregate mixtures \#3 and \#4 utilized H-POWER combined ash and Florida crushed concrete. Although they are not within the scope of the overall project, Permabase, Inc. felt that these combinations offer an opportunity for the city and county of 


\section{Findings}

Results of the HDOT testing procedures appear in Table 2, Appendix B4. Note that all of the results are within the required specifications. This clearly demonstrates that processed H-POWER ash can be successfully combined with locally available natural aggregates or recycled materials to meet all specifications as detailed in Section 308 - Portland Cement Treated Base, of the Hawaii Department of Transportation Standard Specifications for Road and Bridge Construction.

\section{Task 2 Conclusions from Findings}

Task 2 results, like those of Task 1, clearly demonstrate that MWC ash could feasibly function as a quality alternative aggregate. The test results underscore the fact that MWC ash is a chemically safe and physically appropriate material to use in aspects of road construction. With minimal processing, H-POWER combined ash could be easily combined with local aggregates to produce a reliable, economical aggregate mixture. 


\section{Conclusion}

The benefits of utilizing MWC ash in landfill and road construction applications are vast.

In Task 1, the chemical analysis shows no indication that using any of the ash mixtures would cause endangerment of human health or the environment when used as an alternative daily landfill cover. A health risk assessment will be conducted in Phase II to confirm this. The strong buffering capacity coupled with the natural pozzolanic type reactions of wet combined ash should decrease the potential for metal leachability. The addition of sewage sludge to the ash mixtures provides an alternative means for its disposal and should help reduce the moisture and the odor properties of the sludge, resulting in improved public health and environmental consequences as compared to current practices for sludge disposal. Although the chemical analysis and botanical tests show mixtures containing sludge to be less than desirable final top cover material by themselves, these mixtures may still be used as infiltration and erosion layers of final landfill covers. For economic reasons, mixture \#3 (combined H-POWER ash) was chosen for further study in Phase II.

In Task 2, the results of the sieve size analysis indicate that H-POWER combined ash would be best suited for use in asphalt mixtures since over 508 of its particles fall into the minus \#16 size range. Actual tests on mixtures employing H-POWER ash show that it can be used successfully. Additional tests are currently being conducted by Hawaiian Bitumuls and will be reported in Phase II.

As shown, MWC ash is a safe and suitable alternative. It is readily available, and with proper processing, is the key to closing the municipal waste recycling loop. Moreover, MWC ash utilization would extend the current landfill life. Decreasing the amount of material going to the landfill would also mean lower tipping fees at waste-to-energy plants. Alternatively, money currently spent landfilling could be channeled into other areas of waste management. Large scale studies planned for Phase II will be more illustrative of the benefits that MWC ash utilization can bring to municipalities nationwide. 


\section{Acknowledgment}

We would like to express our gratitude and appreciation to the following for their contribution to the project. The management and personnel of: Honolulu Resource Recovery Venture, Ogden Projects Inc., David Kawamoto and Chris steele of Grace Pacific Corporation, Ralph Witham and David Scott of Hawaiian Bitumuls \& Paving Co., Eric Shafer of Permabase Inc., Foremost Dairy, Barbers Point AES, City and County of Honolulu Department of Wastewater Management, Waipahu Incinerator and Material Testing Lab, Dr. David and Teresa Kosson, Dr. Peter Nicholson, and the Hawaii state Department of Health - Office of Solid Waste Management. Finally, we would like to thank the City's H-POWER staff for their assistance in completing this report: Mr. Nathan Yuen, Ms. Valerie Kane, and Mr. Jim Grogan. 


\section{Regulatory Requirements for Landfill Cover Materials}

Sections 11-58.1-15 and 11-58.1-17 of the Hawaii Administrative Rules specify the operational criteria and closure care requirements for material used for daily and final cover for landfills containing municipal solid waste. The landfill daily cover requirements as specified in subsection 58.1-15 include:

1. Cover MSW with at least $15.24 \mathrm{~cm}$. ( 6 inches) of earthen material at the end of each working day, or more often if necessary to control disease vectors, fires, odors, blowing litter, and scavenging, or;

2. Alternative cover material may be approved if the owner or operator demonstrates that the alternative material and thickness can do the same as (1) above without endangering human health and the environment.

Subsection 58.1-17 of the Hawaii Administrative Rules specify requirements at $M S W$ landfills where final cover material is used for final closure. Those regulations indicate that the final cover system must be designed to minimize infiltration and erosion. The infiltration and erosion inhibiting cover layer must:

1. Have a permeability no greater than $1 \times 10^{-5} \mathrm{~cm} / \mathrm{sec}$, and

2. Minimize infiltration through the use of an infiltration layer that contains a minimum $45.72 \mathrm{~cm}$. (18 inches) of earthen material, and

3. Minimize erosion by the use of an erosion layer that contains a minimum of $15.24 \mathrm{~cm}$. ( 6 inches) of earthen material that is capable of sustaining plant growth.

Alternative final cover design to the above requirements may be approved if it is shown that the alternative achieves an equivalent degree of impermeability, and controls infiltration and erosion to a similar or improved degree. 
Appendix A2

Ash Collection, Storage, and Mixture Preparation

Ash Collection and Storage

The H-POWER bottom ash after falling from the boiler grate into a quench tank, is retrieved via the submerged scraper conveyors (SSC) and is deposited onto a conveyor belt which feeds the BAMR. A photograph of the BAMR is shown appears as Figure 1 below.

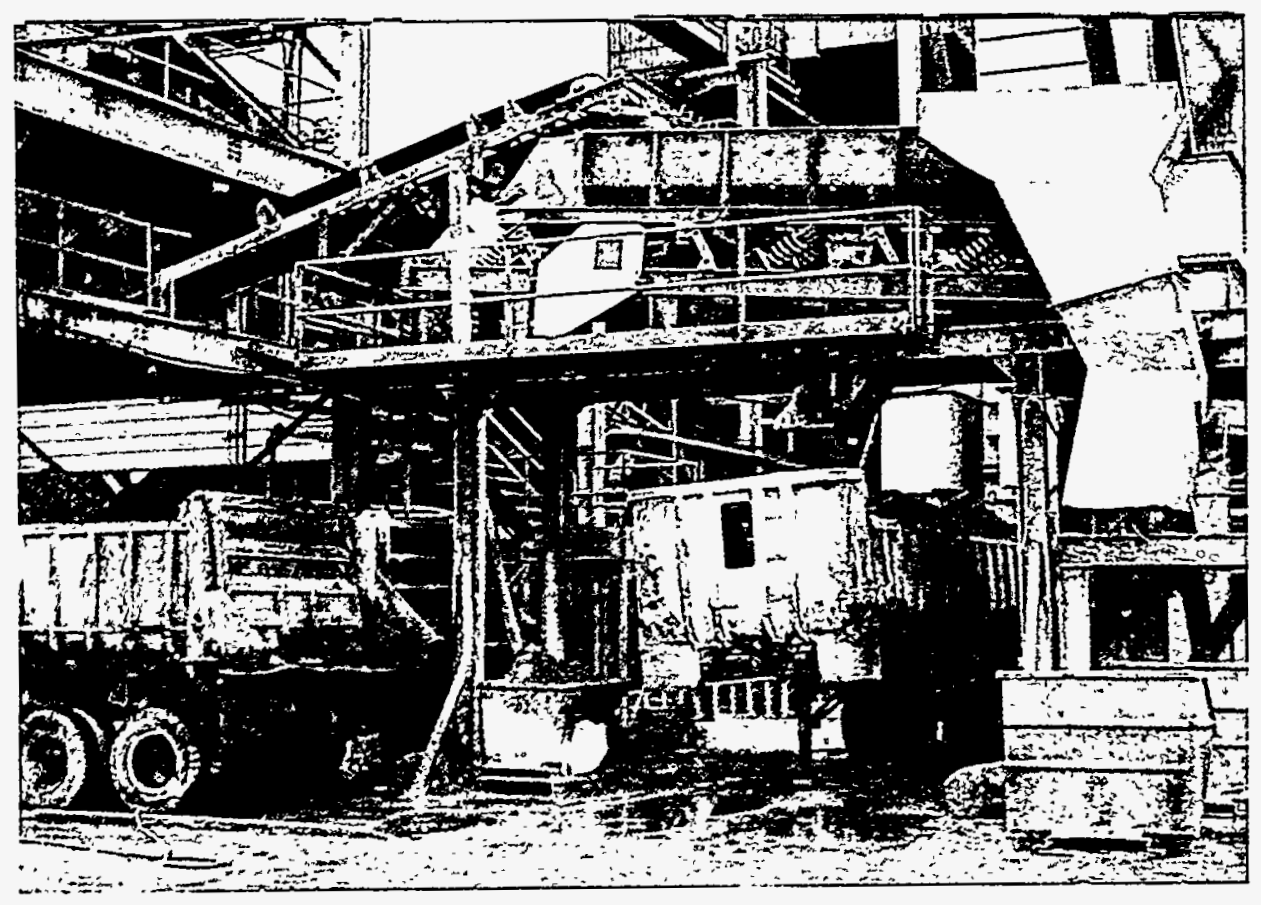

Figure 1

The BAMR is made up of 6 major components: a vibrating finger screen, rotating drum electromagnet, eddy current separator, and three hoppers. The ash stream first encounters the vibrating finger screen. The finger screen has triangular openings with a length of about 3.8 centimeters (1.5 inches) and a base width of roughly 1.6 centimeters (5/8 of an inch). Material which does not pass through the screen continues on past the rotating drum electromagnet which deflects the ferrous metals into a separate hopper. The remaining non-ferrous material is then removed using the eddy current separator which deflects the material into the appropriate hopper. The remaining material is collected in yet a third hopper. Material passing the screen falls directly into the loadout trailer, which is where the samples were collected. 
Six 38-liter (10-galion) samples were taken throughout the day at roughly 1.5 hour intervals.

The H-POWER fly ash comes from the electrostatic precipitators (ESP) and goes into a pug mill where it is sprayed with water to control dusting. The moistened fly ash is then fed into the ash loadout trailer. Since the object was to obtain dry fly ash, the ash was collected prior to reaching the pug mill. Figure 2 below shows the surge bin from which the dry ash was collected.

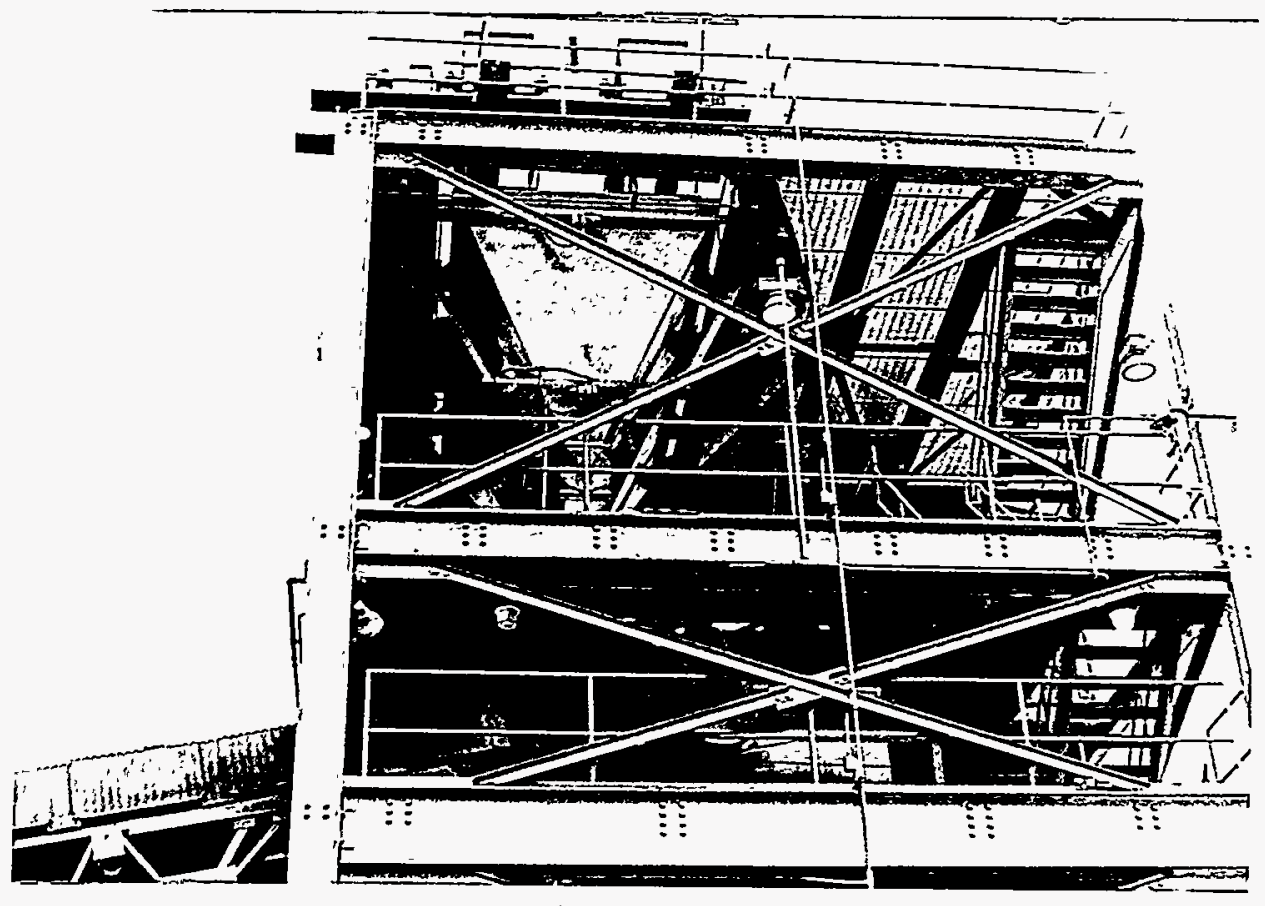

Figure 2

All of the H-POWER ash was placed in the appropriate storage drum and the particulars of collection recorded. Tables 1-5, beginning on page A2-4, show the ash collection data.

Waipahu Incinerator ash was collected from the ash monofill located near the incinerator. Twice each day over a five day period, the ash was taken to the monofill and dumped (see Figures 3 and 4 , page $\mathrm{A} 2-3$ ). The pile was spread out using a Bobcat tractor and samples were taken randomly from different parts of the pile. The ash samples were then loaded from the bucket of the Bobcat into steel storage drums. Table 6 contains the Waipahu ash collection data. 


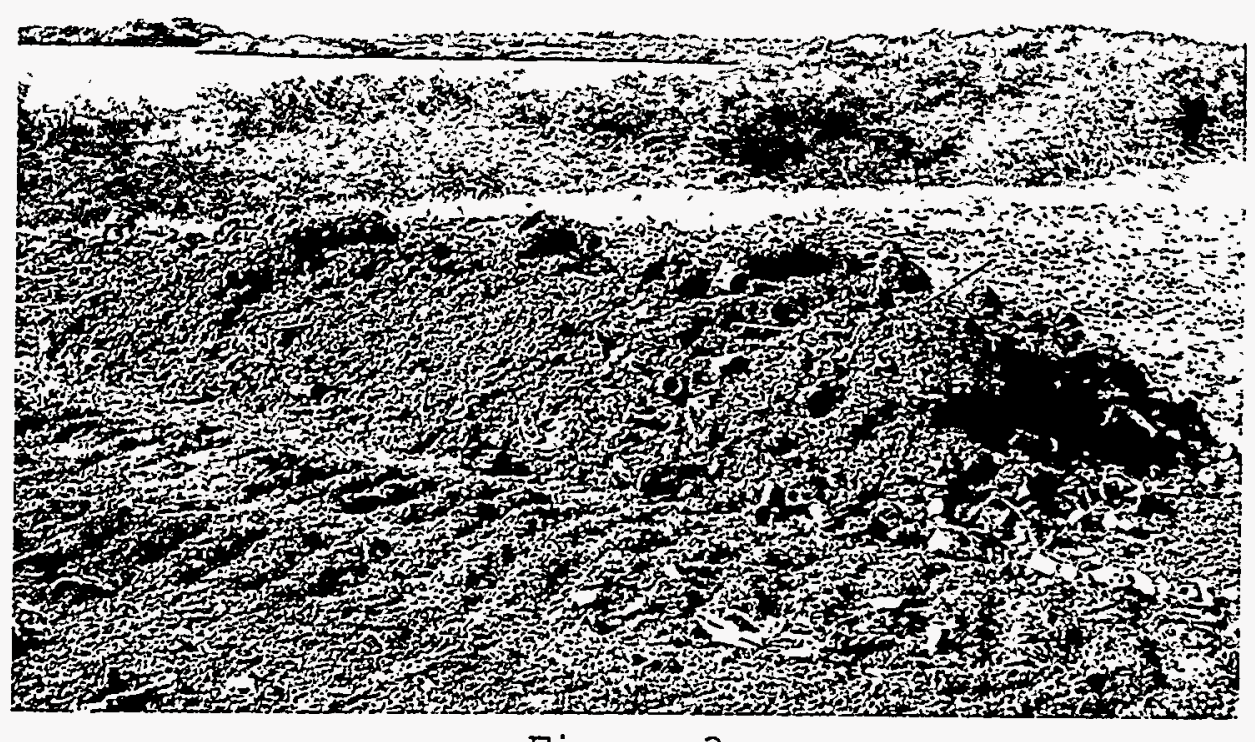

Figure 3

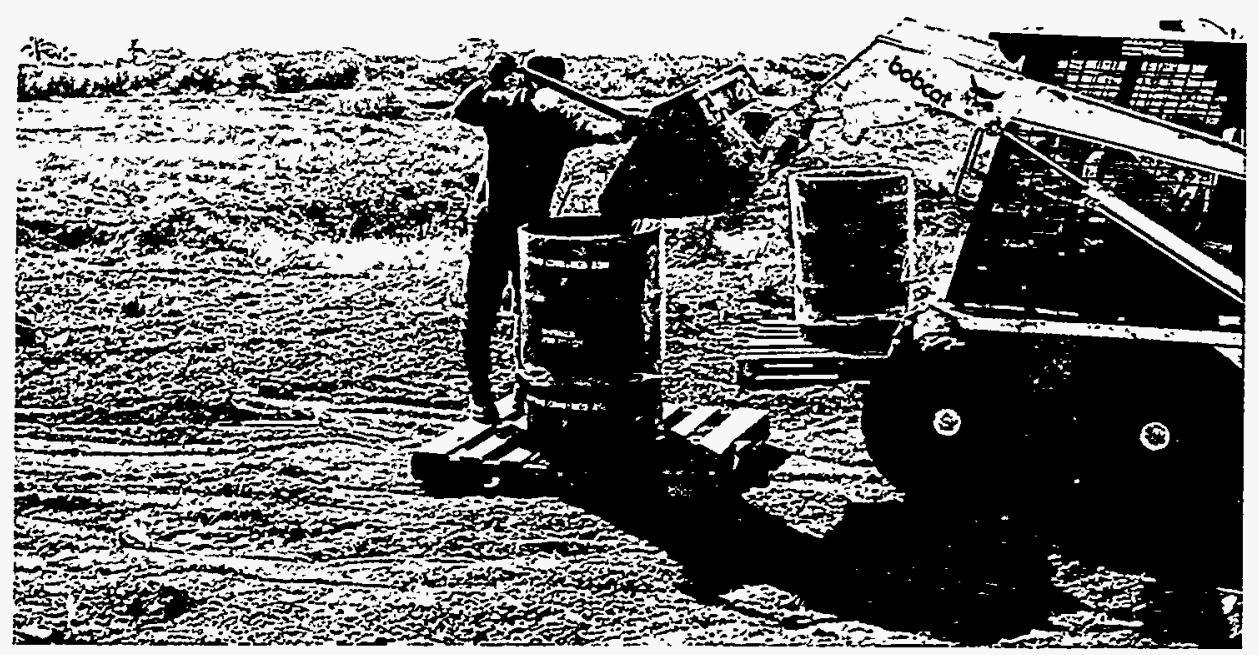

Figure 4 
ASH COLLECTION DATA SHEET (H-POWER FLY ASH)

\begin{tabular}{|c|c|c|c|}
\hline DATE & TIME & $\begin{array}{c}\text { DRUM } \\
\text { NUMBER }\end{array}$ & REMARKS \\
\hline $19-0 c t-93$ & 09:00 AM & $F 1$ & 5 GALLONS \\
\hline 19-Oct-93 & $09: 45 \mathrm{AM}$ & $\overline{F 1}$ & 5 GALLONS \\
\hline $19-0 c t-93$ & 12:00 PM & F1 & 5 GALLONS \\
\hline $19-0 c t-93$ & 01:20 PM & $\overline{F 1}$ & 5 GALLONS \\
\hline 19-Oct-93 & $02: 30 \mathrm{PM}$ & $\mathrm{F1}$ & 5 GALLONS \\
\hline $20-0 c t-93$ & 09:00 AM & $F 2$ & 5 GALLONS \\
\hline $20-0 c t-93$ & $10: 45 \mathrm{AM}$ & F2 & 5 GALLONS \\
\hline $20-0 c t-93$ & 12:15 PM & F2 & 5 GALLONS \\
\hline $20-0 c t-93$ & $01: 15$ PM & F2 & 5 GALLONS \\
\hline $20-0 c t-93$ & 02:15 PM & F2 & 10 GALLONS \\
\hline 21-Oct-93 & $10: 45 \mathrm{AM}$ & F3 & 15 GALLONS \\
\hline 21-Oct-93 & $01: 45 \mathrm{PM}$ & F3 & 15 GALLONS \\
\hline $22-$ Oct-93 & $10: 00 \mathrm{AM}$ & $\overline{F 4}$ & 15 GALLONS \\
\hline $22-0 c t-93$ & 01:30 PM & $\overline{F 4}$ & 15 GALLONS \\
\hline 26-Oct-93 & 12:00 PM & F5 & 15 GALLONS \\
\hline $26-0 c t-93$ & $02: 30 \mathrm{PM}$ & F5 & 15 GALLONS \\
\hline
\end{tabular}

Table 1 
ASH COLLECTION DATA SHEET (H-POWER BOTTOM ASH)

\begin{tabular}{|c|c|c|c|}
\hline DATE & TIME & $\begin{array}{c}\text { DRUM } \\
\text { NUMBER }\end{array}$ & REMARKS \\
\hline $19-0 \mathrm{ct}-93$ & 08:00 AM & $\mathrm{B} 1$ & 5 GALLONS \\
\hline $19-0 c t-93$ & $09: 20 \mathrm{AM}$ & B1: & 5 GALLONS \\
\hline $19-$ Oct-93 & $11: 45 \mathrm{AM}$ & B1 & 5 GALLONS \\
\hline $19-0 \mathrm{ct}-93$ & 01:00 PM & B1 & 5 GALLONS \\
\hline $19-0 c t-93$ & $01: 45$ PM & B1 & 5 GALLONS \\
\hline 19-Oct-93 & $03: 00 \mathrm{PM}$ & B1 & 5 GALLONS \\
\hline $20-0 c t-93$ & $08: 30 \mathrm{AM}$ & $\mathrm{B} 2$ & 5 GALLONS \\
\hline $20-0 c t-93$ & $10: 15 \mathrm{AM}$ & $\overline{\mathrm{B} 2}$ & 5 GALLONS \\
\hline $20-0 c t-93$ & $12: 00 \mathrm{PM}$ & B2 & 5 GALLONS \\
\hline $20-0 c t-93$ & 01:00 PM & $\mathrm{B} 2$ & 5 GALLONS \\
\hline $20-0 c t-93$ & 02:00 PM & $\overline{B 2}$ & 5 GALLONS \\
\hline $20-0 c t-93$ & 03:00 PM & $\mathrm{B2}$ & 5 GALLONS \\
\hline $21-$ Oct-93 & $10: 30 \mathrm{AM}$ & B3 & 5 GALLONS \\
\hline $21-$ Oct-93 & $11: 10 \mathrm{AM}$ & $\overline{B 3}$ & 5 GALLONS \\
\hline $21-$ Oct-93 & $11: 50 \mathrm{AM}$ & B3 & 5 GALLONS \\
\hline $21-$ Oct-93 & 12:30 PM & B3 & 5 GALLONS \\
\hline 21-Oct-93 & 01:10 PM & B3 & 5 GALLONS \\
\hline 21-Oct-93 & 02:1.0 PM & B3 & 5 GALLONS \\
\hline 22-Oct-93 & $08: 30 \mathrm{AM}$ & $\mathrm{B} 4$ & 5 GALLONS \\
\hline $22-$ Oct-93 & $09: 40$ AM & $\overline{B 4}$ & 5 GALLONS \\
\hline $22-$ Oct-93 & $10: 50 \mathrm{AM}$ & B4 & 5 GALLONS \\
\hline $22-0 c t-93$ & 12:00 PM & $\mathrm{B} 4$ & 5 GALLONS \\
\hline $22-$ Oct-93 & $01: 10 P M$ & $\overline{B 4}$ & 5 GALLONS \\
\hline $22-0 c t-93$ & $02: 30 \mathrm{PM}$ & B4 & 5 GALLONS \\
\hline
\end{tabular}

Table 2 


\section{ASH COLLECTION DATA SHEET (H-POWER BOTTOM ASH)}

\begin{tabular}{|c|c|c|c|}
\hline DATE & TIME & $\begin{array}{c}\text { DRUM } \\
\text { NUMBER }\end{array}$ & REMARKS \\
\hline $28-0 c t-93$ & 09:30 AM & B5 & 5 GALLONS \\
\hline $28-0 c t-93$ & $10: 30 \mathrm{AM}$ & $\overline{B 5}$ & 5 GALLONS \\
\hline 28-Oct-93 & $11: 30 \mathrm{AM}$ & $\overline{\mathrm{B} 5}$ & 5 GALLONS \\
\hline 28-Oct-93 & 1.2:30 PM & B5 & 5 GALLONS \\
\hline $28-0 c t-93$ & 01:30 PM & B5 & 5 GALLONS \\
\hline 28-Oct-93 & $02: 30 \mathrm{PM}$ & $\mathrm{B}^{\prime}$ & 5 GALLONS \\
\hline
\end{tabular}

Table 3 
ASH COLLECTION DATA SHEET (H-POWER MIXED ASH)

\begin{tabular}{|c|c|c|c|}
\hline DATE & TIME & \begin{tabular}{|c|} 
DRUM \\
NUMBER
\end{tabular} & REMARKS \\
\hline 19-Oct-93 & $08: 00 \mathrm{AM}$ & M1 & 5 GALLONS BOTTOM \\
\hline 19-Oct-93 & 09:00 AM & M1 & 5 GALLONS FLY \\
\hline 19-Oct-93 & 09:20 AM & M1 & 5 GALLONS BOTTOM \\
\hline 19-Oct-93 & 09:45 AM & M1 & 5 GALLONS FLY \\
\hline $19-0 c t-93$ & $11: 45 \mathrm{AM}$ & $\overline{M 1}$ & 5 GALLONS BOTTOM \\
\hline $19-0 c t-93$ & 12:00 PM & M1 & 5 GALLONS FLY \\
\hline $19-0 c t-93$ & 01:00 PM & $\overline{M 1}$ & 5 GALLONS BOTTOM \\
\hline $19-0 c t-93$ & 01:20 PM & M1 & 5 GALLONS FLY \\
\hline $19-0 c t-93$ & $01: 45$ PM & M1 & 5 GALLONS BOTTOM \\
\hline 19-Oct-93 & 03:00 PM & M1 & 5 GALLONS BOTTOM \\
\hline $20-$ Oct-93 & $08: 30 \mathrm{AM}$ & $\mathrm{M} 2$ & 5 GALLONS BOTTOM \\
\hline $20-$ Oct-93 & 09:00 AM & M2 & 5 GALLONS FLY \\
\hline $20-0 c t-93$ & $10: 15 \mathrm{AM}$ & $M 2$ & 5 GALLONS BOTTOM \\
\hline $20-0 c t-93$ & $10: 45 \mathrm{AM}$ & $\mathrm{M} 2$ & 5 GALLONS FLY \\
\hline $20-0 c t-93$ & 12:00 PM & M2 & 5 GALLONS BOTTOM \\
\hline $20-0 c t-93$ & 12:15 PM & $\mathrm{M} 2$ & 5 GALLONS FLY \\
\hline $20-0 c t-93$ & 01:00 PM & M2 & 5 GALLONS BOTTOM \\
\hline $20-0 c t-93$ & $01: 15$ PM & $\mathrm{M} 2$ & 5 GALLONS FLY \\
\hline $20-0 c t-93$ & $02: 00 \mathrm{PM}$ & M2 & 5 GALLONS BOTTOM \\
\hline $20-$ Oct-93 & 03:00 PM & M2 & 5 GALLONS BOTTOM \\
\hline $21-$ Oct-93 & 10:30 AM & M3 & 5 GALLONS BOTTOM \\
\hline $21-0 c t-93$ & $10: 45 \mathrm{AM}$ & $\overline{M 3}$ & 10 GALLONS FLY \\
\hline 21-Oct-93 & $11: 10 \mathrm{AM}$ & M3 & 5 GALLONS BOTTOM \\
\hline $21-$ Oct-93 & $11: 50 \mathrm{AM}$ & M3 & 5 GALLONS BOTTOM \\
\hline $21-$ Oct-93 & 12:30 PM & M3 & 5 GALLONS BOTTOM \\
\hline $21-$ Oct-93 & 01:10 PM & M3 & 5 GALLONS BOTTOM \\
\hline $21-$ Oct-93 & $01: 45 \overline{P M}$ & M3 & 10 GALLONS FLY \\
\hline $21-$ Oct-93 & $02: 10 P M$ & M3 & 5 GALLONS BOTTOM \\
\hline
\end{tabular}

Table 4 


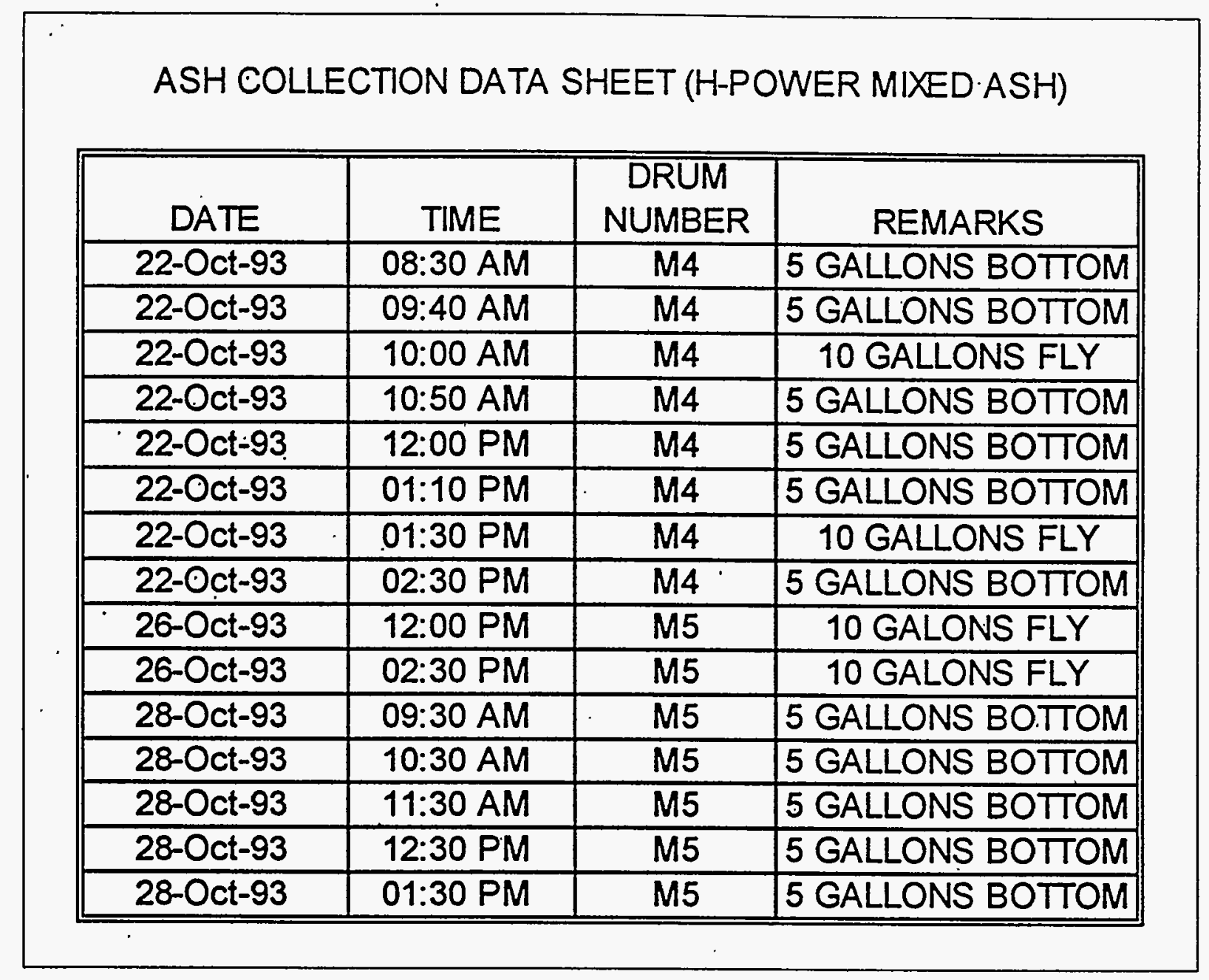

Table 5 
ASH COLLECTION DATA SHEET (WAIPAHU INCINERATOR)

\begin{tabular}{|c|c|c|c|}
\hline DATE & TIME & $\begin{array}{c}\text { DRUM } \\
\text { NUMBER }\end{array}$ & REMARKS. \\
\hline $18-0 \mathrm{ct}-93$ & $09: 30 \mathrm{AM}$ & W1 & COLLECTION FROM 7 DIFFERENT AREAS \\
\hline $18-0 c t-93$ & 12:30 PM & $\mathrm{W} 2$ & COLLECTION FROM 6 DIFFERENT AREAS \\
\hline $19-0 c t-93$ & 09:30 AM & W3 & COLLECTION FROM 5 DIFFERENT AREAS \\
\hline 19-0ct-93 & 12:30 PM & W4 & COLLECTIONFROM 6 DIFFERENT AREAS \\
\hline $20-0 c t-93$ & 09:30 AM & W5 & COLLECTION FROM 6 DIFFERENT AREAS \\
\hline $20-0 c t-93$ & 12:30 PM & W6 & COLLECTION FROM 6 DIFFERENT AREAS \\
\hline & & & - \\
\hline $21-0 c t-93$ & 09:30 AM & W7 & COLLECTON FROM 6 DIFFERENT AREAS \\
\hline $21-0 c t-93$ & 12:30 PM & W8 & COLLECTION FROM 6 DIFFERENT AREAS \\
\hline & & & \\
\hline $22-0 c t-93$ & 09:30 AM & W9 & COLLECTION FROM 7 DIFFERENT AREAS \\
\hline 22-0ct.93 & $12: 30 P M$ & W10 & COLLECTION FROM 7 DIFFERENT AREAS. \\
\hline
\end{tabular}

Table 6 
19 liters ( 5 gallons) from which to sample for chemical and microbiological analysis. 
Appendix A3

Chemical Analysis of Ash Mixtures

Task 1 chemical analysis was carried out following the methods listed below:

Task 1 Chemical Analysis Methods

$\begin{array}{ll}\text { Aluminum } & \text { EPA Method } 3050 \mathrm{M} \\ \text { Arsenic } & \text { EPA Method } 6010 \\ \text { Barium } & \text { EPA Method } 6010 \\ \text { Cadmium } & \text { EPA Method } 6010 \\ \text { Calcium } & \text { EPA Method } 6010 \\ \text { Chromium } & \text { EPA Method } 6010 \\ \text { Copper } & \text { EPA Method } 6010 \\ \text { Iron } & \text { EPA Method } 6010 \\ \text { Lead } & \text { EPA Method 6010 } \\ \text { Mercury } & \text { EPA Method } 6010 \\ \text { Potassium } & \text { EPA Method } 6010 \\ \text { Selenium } & \text { EPA Method } 6010 \\ \text { Silicon } & \text { EPA Method } 6010 \\ \text { Silver } & \text { EPA Method } 6010 \\ \text { Zinc } & \text { EPA Method } 6010 \\ \text { pH } & \text { EPA Method } 9045 \\ \text { Chloride } & \text { EPA Method } 325.3 \\ \text { Sulfate } & \text { EPA Method } 375.4 \\ \text { Carbon } & \text { ASTM D5291M } \\ \text { Nitrate/Nitrite } & \text { EPA Method } 353.3 \\ \text { Total Nitrogen } & \text { EPA Method } 351.3 \\ \text { Moisture } & \text { CLP ILM 2.0 }\end{array}$

Inductively coupled argon plasma analysis (ICAP) was used for all metals except silicon where atomic absorption spectroscopy methods were used.

\section{Summary Findings}

The following is a detailed summary of the Task 1 chemical analysis findings. Please refer to the appropriate tables listed at the beginning of each section. Data appearing in the tables has been adapted from the E.L. Pacific laboratory report, which can be found beginning on page A3-13 of this appendix. 
In addition to comparing the elemental chemistry data from each of these mixtures with each other, it is also useful to compare the concentrations of H-POWER fly ash only (mix 1) and H-POWER bottom ash only (mix 2) to the fly ash and bottom ash concentrations EPA found in a 1991 study it conducted at the Mid-Connecticut (MC) RDF facility (Appendix C1). That facility burns similar prepared fuel (RDF) and is nearly identical in design except in that it employs a baghouse instead of an electrostatic precipitator (ESP) for particulate control.

Aluminum through Chromium

Refer to Table I on page A3-4

Aluminum - Low: 7,884.5 PPM, High: 55,052.5 PPM. Except for H-POWER bottom ash, the aluminum levels were higher in the soil control mixtures than any ash mixtures. The average concentration of aluminum in the H-POWER bottom ash was much higher at 55,052 PPM than in the fly ash which averaged 7,884 PPM. The aluminum bottom ash concentrations at H-POWER are essentially the same as that which EPA found at the Mid Connecticut RDF facility (MC) in their 1991 study, but the fly ash concentrations were much lower than the 59, 300 PPM at MC.

Arsenic - Low: Non-detect, High: 75.5 PPM (mix $1^{1}$ ). There was no significant difference, however, between the arsenic concentration in the control and any other mixture except mixture 1. The fly ash arsenic concentration was slightly higher than the MC fly ash which averaged 19 PPM, but the bottom ash which had no detectable concentrations 10.5 PPM detection limit) was lower than MC's 10 PPM.

Barium - Low: 128.5 PPM, High: 690 PPM (mix 2). The range of variation was very narrow and one can not conclude there was any appreciable increase in barium in the ash mixtures over the control. The barium concentrations in MC fly ash at an average 98 PPM was lower than H-POWER's average of 310 PPM. The bottom ash at H-POWER was also slightly higher (690 PPM) than at MC which averaged 403 PPM.

Cadmium -Low: Non-detect (control), High: 58 PPM. This also was a very narrow range of variation and no meaningful difference can be drawn between concentration in the ash mixtures than in the control mixture. The H-POWER fly ash concentrations which averaged 27 PPM were lower than MC

A table summarizing mixture contents appears on page A3-12 (table 9) of this appendix. 
which averaged about 100 PPM. Similarly the H-POWER non-detectable concentrations (0.05 PPM detection limit) in the bottom ash were lower than MC's bottom ash concentrations (7. PPM).

Calcium - Low: 30,133 PPM (control), High: 148,901 PPM (mix 8). This 5-fold increase compared to the control mixture is not surprising. The similarity in the fly and bottom ash concentrations (each averaging around 104,000 PPM) was peculiar, however, and is so far inexplicable. Also a surprise was the higher MC concentrations $(120,000$ PPM). This is surprising because H-POWER, having a dry lime scrubber and an ESP, was expected to have a much higher fly ash calcium concentration (MC also burns RDF and has a dry lime scrubber, but with a baghouse fabric filter instead of an ESP). This is because an ESP requires more calcium to reduce $\mathrm{SO}_{2}$ levels than with a baghouse. The unreacted lime that coats the bags in a baghouse allows additional time to react with the gaseous $\mathrm{SO}_{2}$ to form calcium sulfate.

Chromium - Low: 37 PPM (mix 8), High: 280 PPM (control). The control mixture, with an average concentration of 280 PPM, was slightly higher than either the average concentrations for H-POWER fly or bottom ash (118 and 187 PPM). The MC bottom ash concentration averaged around 200 PPM and about $220 \mathrm{PPM}$ in the fly ash.

Table 1 below shows the results for $\mathrm{Al}, \mathrm{As}, \mathrm{Ba}, \mathrm{Cd}, \mathrm{Ca}$, and $\mathrm{Cr}$ for each of the duplicate mixtures. An average is calculated for each of the six chemical parameters listed above for each mixture. The control mixture, $\operatorname{mix} 4$, is a sample of native soil currently used as daily cover taken from the Waimanalo Gulch landfill. At the bottom of the table, an average concentration for all mixtures excluding this control is given. The percent difference between the mixture average and the control average is then calculated according to the following formula:

$$
\% \text { diff }=\frac{\text { mix avg }- \text { control avg }}{\text { mix avg }}
$$

Tables 2, 3, and 4 are set up the same way for the remaining chemical parameters. 


\begin{tabular}{|c|c|c|c|c|c|c|}
\hline \multicolumn{7}{|c|}{ Chemical Analysis in units of PPM unless otherwise indicated } \\
\hline Test Mix & Al & As & $\mathrm{Ba}$ & Cd & $\mathbf{C a}$ & $C_{r}$ \\
\hline Mox 1a & 10233.00 & 76.00 & 310.00 & 26.00 & 107248.00 & 116.00 \\
\hline $\operatorname{Ma} \times 1 b$ & 550.00 & 75.00 & 310.00 & $27 . \infty$ & 90844.00 & 120.00 \\
\hline Mix 1 avg. & 7884.50 & 75.50 & 310.00 & 26.50 & 103546.00 & 118.00 \\
\hline $\operatorname{Ma} \times 2 a$ & 48905.00 & 25 & 570.00 & $25^{*}$ & 1001200 & 160.00 \\
\hline $\operatorname{Mix} \mathbf{2}$ & 61120.00 & 25 & 810.00 & $25^{\circ}$ & 10100200 & $214 . \infty$ \\
\hline Mix 2 avg. & 55052.50 & 25.00 & 690.00 & 250 & 10510200 & 187.00 \\
\hline Mx $3 a$ & 46341.00 & $25^{*}$ & $5^{*}$ & 11.00 & 131156.00 & $206 . \infty$ \\
\hline Mx 30 & 47409.00 & 87.00 & 537.00 & 105.00 & 118166.00 & $181 . \infty$ \\
\hline Mix 3 avg. & 46905.00 & 56.00 & 271.00 & 58.00 & 124661.00 & 193.50 \\
\hline $\operatorname{Mix} 4 a$ & 5563.00 & $25^{*}$ & 184.00 & $25^{*}$ & 24145.00 & 250.00 \\
\hline $\operatorname{Mix} 4 b$ & 50755.0 & $25^{\circ}$ & 73.00 & 25 & 36121.00 & 310.00 \\
\hline MIX 4 avg. [Control, (CT)] & 53159.00 & 25.00 & 128.50 & 2.50 & 30133.00 & 280.00 \\
\hline Mix 5a & 26877.00 & 73.00 & 405.00 & 23.00 & 112523.00 & $113 . \infty$ \\
\hline$M \times 5 b$ & 26758.00 & 74.00 & 360.00 & 24.00 & 123589.00 & 110.00 \\
\hline MIX 5 ang. & 26817.50 & 73.50 & 38250 & 23.50 & 118171.00 & 111.50 \\
\hline $\operatorname{Mix} 6 a$ & 41040.00 & 25 & 238.00 & 7.00 & 81646.00 & 55.00 \\
\hline $\operatorname{Mx} \infty$ & 23197.00 & $25^{*}$ & 220.00 & 5.00 & 75629.00 & 46.00 \\
\hline Mix 6 avg. & 32118.50 & 25.00 & 229.00 & 6.00 & 78637.50 & 50.50 \\
\hline $\operatorname{Max} 7 a$ & 16035.00 & 25 & 233.00 & 16.00 & 101851.00 & 83.00 \\
\hline$M \times 7 b$ & 19585.00 & $\mathbf{2 5}^{*}$ & 270.00 & 15.00 & 94160.00 & 84.00 \\
\hline Mix 7 ang. & 18210.00 & 25.00 & 251.50 & 15.50 & 98010.50 & 83.50 \\
\hline 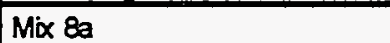 & 10485.00 & $25^{*}$ & 139.00 & $8 . \infty$ & 145000.00 & 39.00 \\
\hline $\operatorname{Mix} 8 \infty$ & 10003.00 & 25 & $119 . \infty 0$ & 7.00 & 15480200 & 35.00 \\
\hline Mil 8 ang. & 10394.00 & 25.00 & 129.00 & 7.50 & 148901.00 & 37.00 \\
\hline Mix $9 a$ & 49099.00 & 70.00 & 391.00 & 25.00 & 94776.00 & 20200 \\
\hline$M \times 90$ & 50794.00 & 25 & 303.00 & 20.00 & 79435.00 & 214.00 \\
\hline Mix 9 avg. & 49946.50 & 47.50 & 347.00 & 2250 & 87105.50 & 208.00 \\
\hline Avg all mix except CT & 30916.06 & 44.06 & 326.25 & 20.25 & 100016.81 & 123.63 \\
\hline Avg $f \circ C T$ & 53159.00 & 25.00 & 128.50 & 250 & 30133.00 & 280.00 \\
\hline Percent difference & $-71.05 \%$ & $43.25 \%$ & $60.61 \%$ & $87.65 \%$ & $72.10 \%$ & $-126.49 \%$ \\
\hline
\end{tabular}

Table 1

Copper through selenium

Refer to Table 2 on page A3-6

Copper - Low: 79 PPM (mix 6), High: 2,842.5 PPM (mix 3).

The control concentrations ( 98.5 PPM), appear to be at least 1 order of magnitude lower than the H-POWER bottom and combined ash which averaged about 2,000 PPM. The H-POWER bottom ash concentration is similar to the MC range of 1,000-6,000 PPM. The fly ash concentration is also similar at 200-600 PPM. 
Iron - Low: 4,787.5 PPM (mix 8), High: 77,125 PPM (mix 9). The second highest average iron concentration (74,995 PPM) was found in the soil control mixture. The MC fly ash concentrations average around 550 PPM compared to H-POWER's 16,000 PPM. The MC bottom ash concentrations were also much lower at around 5000 PPM compared to 38,000 PPM for H-POWER bottom ash.

Lead - Low: 37 PPM (control), High: 9,490.5 PPM (mix 3) This concentration is at least one order of magnitude higher in all the ash mixtures--except those containing sewage sludge--than in the control mixtures. The H-POWER fly ash concentrations are slightly lower at an average 1,784 PPM than the 3,000 PPM average at MC. The H-POWER bottom ash concentrations appear slightly higher at an average 7,788 PPM compared to MC's average of around 2,000 PPM.

Mercury - Low: 8 PPM (mix 9), High: 13.5 PPM (mix 5). The MC fly ash concentrations averaged higher at 38 PPM compared to H-POWER's average of 12 PPM. The MC bottom ash levels were lower at concentrations slightly below 1 PPM compared to H-POWER's average of 9 PPM.

Potassium - Low: 1,212.5 PPM (control), High: 11,515.5 PPM (mix 3). This appears to be another case where all the ash mixtures--except those containing sewage sludge--were at least one order of magnitude higher than the soil control. There is no published data of MC potassium levels.

Selenium - There were no detectable concentrations of selenium found in any mixtures nor were any found in the MC ash samples. Thus there is nothing to indicate any comparative findings for this element. 


\begin{tabular}{|c|c|c|c|c|c|c|}
\hline \multicolumn{7}{|c|}{ Chemical Analysis in urits of PPM unless otherwise indicated } \\
\hline Test Mx & $\mathrm{Cu}$ & $\mathrm{Fe}$ & $\mathrm{Pb}$ & $\mathrm{Hg}$ & $\mathbf{K}$ & Se \\
\hline Mix 1a & $241 . \infty$ & 14953.00 & $1707 . \infty$ & 10.00 & 8640.00 & 25 \\
\hline Mix 1b & 260.00 & 1751200 & $1861 . \infty$ & 14.00 & 8049.00 & 25 \\
\hline Mix 1 avg. & 250.50 & 16232.50 & 1784.00 & 12.00 & 8644.50 & 25.00 \\
\hline $\operatorname{Mix} 2 \mathrm{a}$ & $3800 . \infty$ & 27401.00 & 828.00 & 1200 & 7950.00 & 25 \\
\hline$M \propto 20$ & 940.00 & 50101.00 & 14748.00 & 5.30 & 7550.00 & $25^{\circ}$ \\
\hline Mlx 2 avg. & 2370.00 & 38751.00 & 7788.00 & 8.65 & $\pi 57.50$ & 25.00 \\
\hline Mix 3a & $5126 \infty$ & 25350.00 & 317200 & 8.20 & 10800.00 & 25 \\
\hline Mix 30 & 50.00 & $24782 \infty$ & 15809.00 & 7.70 & 12231.00 & $\mathbf{2 5}$ \\
\hline Mlx 3 ang. & 284250 & 25081.00 & 9490.50 & 7.95 & 11515.50 & 25.00 \\
\hline Mix 4a & $\$ 5.00$ & 64255.00 & 48.00 & 5.20 & 139300 & 25 \\
\hline Mix $4 b$ & 10200 & 85156.00 & 26.00 & 1200 & 100200 & 25 \\
\hline Mix 4 ang. [Control, (CT)] & 98.50 & 74995.50 & 37.00 & 8.60 & 121250 & 25.00 \\
\hline Mix $5 a$ & 245.00 & 13874.00 & 1437.00 & 14.00 & 8457.00 & $25^{*}$ \\
\hline Ma 50 & 275.00 & 15150.00 & 158200 & 13.00 & 8574.00 & $\mathbf{2 5}$ \\
\hline Mix 5 avg. & 260.00 & 14512,00 & 1509.50 & 13.50 & 8515.50 & 25.00 \\
\hline$M \times 6 a$ & 78.00 & 16815.00 & 509.00 & $12 \infty$ & 5056.00 & 25 \\
\hline $\operatorname{Mx} \mathbf{6 b}$ & 80.00 & 15261.00 & 460.00 & 11.00 & $5770 . \infty$ & 25 \\
\hline Mix $6 \mathrm{ang}$. & 79.00 & 16038.00 & 514.50 & 11.50 & 5418.00 & 25.00 \\
\hline $\mathrm{M} \times \mathrm{7a}$ & 199.00 & 100740.00 & 1150.00 & 1200 & 5811.00 & 25 \\
\hline $\operatorname{Mix} 7 \mathrm{~b}$ & 200.00 & 13008.00 & 840.00 & 10.00 & 6111.00 & 25 \\
\hline Mix 7 ang. & 209.50 & 56914.00 & 995.00 & 11.00 & 5961.00 & 25.00 \\
\hline Mix 89 & 98.00 & 474200 & 447.00 & $10 . \infty$ & 3511.00 & 25 \\
\hline Max \&b & 76.00 & 4833.00 & 348.00 & 13.00 & 3298.00 & $25^{\circ}$ \\
\hline MIX 8 avg. & 87.00 & 4787.50 & 397.50 & 11.50 & 3404.50 & 25.00 \\
\hline $\mathrm{Mx} \times \mathrm{Sa}$ & 464.00 & $50132 \infty$ & 919.00 & 5.00 & 9716.00 & 25 \\
\hline Mx So & 429.00 & 104118.00 & 606.00 & 11.00 & $7717 . \infty$ & $25^{*}$ \\
\hline Mix 9 ang. & 446.50 & 77125.00 & 762.50 & 8.00 & 8716.50 & 25.00 \\
\hline Avg all mix except CT & 818.13 & 31180.13 & 2905.19 & 10.51 & 7491.63 & 25.00 \\
\hline Avg for CT & 98.50 & 74905.50 & 37.00 & 8.60 & 121250 & $25 . \infty$ \\
\hline Percent difference & $87.95 \%$ & $-140.52 \%$ & $98.73 \%$ & $18.19 \%$ & $83.82 \%$ & $0.00 \%$ \\
\hline
\end{tabular}

Table 2 
Silicon through Sulfates

Refer to Table 3 on page A3-8

Silicon - Low: 18,451.5 PPM (mix 8), High: 224,102 PPM (control). The H-POWER fly ash which averages about 62,000 PPM, is very similar to MC fly ash at 59,000 PPM. The H-POWER bottom ash, which averaged about 164,000 PPM was higher than the MC bottom ash which averaged about 50,000 PPM.

Silver- Low: 2.5 ( $\operatorname{mix} 4$ ), High: 12 PPM (mix 3 ). The soil control concentration averaged 3 PPM. MC fly ash and bottom ash concentrations were similarly low averaging around 1-2 PPM.

Zinc - Low: 119.5 PPM (control), High: 12,819.5 PPM (mix 2). It is clear that the zinc in the ash mixtures have increased by about one order of magnitude compared to the soil controls. The H-POWER fly ash concentrations, which average around 2,000 PPM, are lower than the MC fly ash zinc levels which at 7,000 PPM. The H-POWER bottom ash zinc concentrations, at an average of 12,000 PPM, are higher than MC's which are roughly 1,300 PPM.

pH - The $\mathrm{pH}$ levels of all the ash mixtures were in a consistent range of 11.00 to 11.96 while the control mixtures averaged 8.15. This is probably the most significant chemical difference between the ash mixtures and the control mixtures. The high $\mathrm{pH}$ levels in the ash mixtures can have both positive and negative effects. Since lead becomes more soluble at lower and higher pH levels, there would likely be increased solution rates of lead, accompanying the addition of water to the mixtures. On the other hand the high $\mathrm{pH}$ will help to kill various microorganisms and varmints which might be attracted to sanitary landfills causing bad odors and unsanitary conditions. Moreover, high pH landfill cover mixtures help guard against leaching of metals from the MSW and also provides protection against acid rain.

Chloride - The chloride concentrations, which are expressed as percent by weight, vary from a low of $0.05 \%$ (control), to a high of $2.98(\operatorname{mix} 1)$. This increase is caused by the combination of HCL in the flue gas with $\mathrm{CaOH}$ from the scrubber to form $\mathrm{CaCL}_{2}$. Since HCL concentrations in the RDF can average around $0.5 \%$, it is not surprising to see chloride levels increase 3 times in the fly ash compared to 
the RDF, and 10 times compared to the levels in the soil. These results appear consistent with the elemental or ultimate chloride analysis of RDF found at EPA's MC study.

Sulfates - The percent sulfate concentrations in the mixtures were extremely low, varying from 0.0028 for mix 2, to maximum levels of only 0.128 in $\mathrm{mix} 5$. No statistical significance could be determined between the soil control sulfate levels and the ash mixture levels.

\begin{tabular}{|c|c|c|c|c|c|c|}
\hline \multicolumn{7}{|c|}{ TABLE 5} \\
\hline \multicolumn{7}{|c|}{ Chemical Analysis in units of PPM unless otherwise indicated } \\
\hline Test Mix & SI & $\mathbf{A g}$ & $\mathrm{Zn}$ & $\mathrm{pH}$ & Chloride $(\%)$ & Sulfate $\%$ \\
\hline Mix $1 a$ & 62966.00 & 10.00 & $2106 . \infty$ & 11.69 & 290 & 0.01 \\
\hline Mix 1b & $62747 . \infty$ & $7 . \infty$ & 1700.00 & 11.64 & 260 & $0 . \infty 2$ \\
\hline Mix 1 avg. & 62866.50 & 8.50 & 1903.00 & 11.67 & 275 & 0.01 \\
\hline Mix $2 a$ & 158738.00 & 250 & 23600.00 & 11.91 & 0.26 & $0 . \infty$ \\
\hline Mix $\boldsymbol{x}$ & 16968200 & 250 & $2039 . \infty$ & 11.76 & 0.05 & $0 . \infty$ \\
\hline MIX 2 avg. & 164210.00 & 2.50 & 12819.50 & 11.84 & 0.16 & 0.00 \\
\hline M× 3a & 116705.00 & 8.00 & 20200.00 & 11.88 & $1 . \infty$ & 0.01 \\
\hline Max 30 & 113747.00 & 16.00 & 405200 & 11.85 & 1.37 & 0.01 \\
\hline Mil 3 avg. & 115226.00 & 12.00 & 12146.00 & 11.87 & 1.19 & 0.01 \\
\hline Mix 4a & 174885.00 & 250 & 116.00 & 7.96 & 0.05 & 0.01 \\
\hline Mx 4b & 273319.00 & 250 & 123.00 & 8.33 & 0.11 & 0.01 \\
\hline Mix 4 ang. [Control, (CT)] & 224102.00 & 250 & 119.50 & 8.15 & 0.08 & 0.01 \\
\hline Mx 5a & 56494.00 & 0.00 & 199200 & 11.67 & 250 & 0.03 \\
\hline Mix 50 & 57485.00 & 9.00 & 2119.00 & 11.66 & 1.19 & 0.03 \\
\hline Mix 5 avg. & 56989.50 & 4.50 & 2055.50 & 11.67 & 1.85 & 0.03 \\
\hline$M \times 6 a$ & 62943.00 & 6.00 & 735.00 & 11.94 & 0.37 & 0.12 \\
\hline Mix $\infty$ & 52684.00 & 7.00 & 640.00 & 11.85 & 0.77 & 0.12 \\
\hline Mix 6 avg. & 57813.50 & 6.50 & 687.50 & 11.90 & 0.57 & 0.12 \\
\hline $\operatorname{Mix} 7 a$ & 46204.00 & 11.00 & 1538.00 & 11.81 & 1.80 & 0.02 \\
\hline$M i x \bar{b}$ & 43523.00 & 11.00 & 1468.00 & 11.85 & 1.80 & 0.04 \\
\hline Mix 7 avg. & 44863.50 & 11.00 & 1503.00 & 11.83 & 1.80 & 0.03 \\
\hline $\mathrm{Mx} 8 \mathrm{a}$ & 19570.0 & $7 . \infty$ & 796.00 & $11 . \infty$ & 0.87 & 0.01 \\
\hline$M i x \&$ & 17333.00 & 8.00 & 675.00 & 11.92 & 0.98 & 0.01 \\
\hline Mix 8 avg. & 18451.50 & 7.50 & 735.50 & 11.46 & 0.93 & 0.01 \\
\hline $\operatorname{Mix} \mathrm{Sa}$ & 90357.00 & 6.00 & 303200 & 11.94 & 0.61 & 0.01 \\
\hline$M x$ So & 127800.00 & 5.00 & 3203.00 & 11.96 & 0.58 & 0.01 \\
\hline Mix 9 avg. & 109108.50 & 5.50 & 316250 & 11.95 & 0.60 & 0.01 \\
\hline Avg all mix except CT & 78691.13 & 7.25 & 4376.56 & 11.77 & 1.23 & 0.03 \\
\hline Avg for CT & 22410200 & 250 & 119.50 & 8.15 & 0.08 & 0.01 \\
\hline Percent difference & $-184.79 \%$ & $5.52 \%$ & $97.27 \%$ & Na & $93.36 \%$ & $57.42 \%$ \\
\hline
\end{tabular}

Table 3 
Nitrate through Moisture

Refer to Tables 4 on page A3-10

Nitrate/Nitrite - Low: non-detect, High: $14.5 \%$ (control). The mixtures with sewage sludge were able to bring the nitrate/nitrite levels up to $3-4 \%$. There is no published data from the EPA MC study to compare to the H-POWER ash data, but it is unlikely the MC would have been different.

Total Nitrogen - The total percent nitrogen levels in the mixtures varied in a similar manner to that found above for nitrate/nitrite. The ash mixtures without sewage sludge had no detectable nitrogen levels. The soil control samples averaged 0.028 and mixture 5 had the maximum total nitrogen levels of $0.16 \%$. Nitrogen is essential to all plant and animal life. Thus for final landfill cover nitrogen in the sewage sludge component of some ash mixtures will be effective in improving the ability of the final cover to support plant growth.

Carbon - The percent carbon levels--as determined by loss on ignition--for the various mixtures ranged from a low of $0.51 \%$ for $\mathrm{mix} \mathrm{2,} \mathrm{to} \mathrm{a} \mathrm{high} \mathrm{of} 3.2 \%$ for $\mathrm{mix} 5$. The soil control.samples averaged $0.535 \%$. This low carbon level in bottom ash is good for ash stabilization purposes because it lowers the voids produced over time from bacterial actions or when the ash is heated to high temperatures. The percent carbon in fly ash was found to be about 1.5\%. These levels are very similar to what was found in the EPA MC study.

Moisture - Low: $21 \%(\operatorname{mix} 3)$, High: 60.58 ( $\operatorname{mix} 7$ ). The moisture levels were largely a function of how much water was added to form the mixture. Water was added so that hydration of the cementitious materials would occur and cause the mixtures to harden as they dried. It was not possible to formulate an optimum water content for each mixture, and different mixtures appeared to require different amounts of water. The fly ash mixtures required more water to cause the mixture to become well mixed and pasty. The bottom ash samples required much less water to get to this state, since they were already saturated. When the same amount of water was added to the samples containing sewage sludge, the water was retained in higher levels even after curing because the pozzolanic reactions were much lower in these mixtures, and thus did not use up the water to the same degree. 


\begin{tabular}{|c|c|c|c|c|c|}
\hline \multicolumn{6}{|c|}{ Chemical Analysis in units of PPM unless otherwise indicated } \\
\hline Test Mix & Nitrate/Nitrite & T. Nitrogen & Carbon & Moisture & Density \\
\hline Mix $1 \mathrm{a}$ & $1{ }^{*}$ & $10^{\circ}$ & 1.63 & 4200 & 1.79 \\
\hline Mix ib & 1* & $10^{\infty}$ & 1.75 & 41.00 & 1.69 \\
\hline Mix 1 avg. & 1.00 & 10.00 & 1.69 & 41.50 & 1.74 \\
\hline Mix $2 a$ & $1 *$ & $10^{*}$ & 0.46 & 23.00 & 1.76 \\
\hline Mix $\mathbf{2 b}$ & $1 *$ & $10^{\circ}$ & 0.55 & 20.00 & 2.10 \\
\hline Mix 2 avg. & 1.00 & 10.00 & 0.51 & 21.50 & 1.93 \\
\hline$M \times 3 a$ & $1 *$ & $10^{*}$ & 1.53 & $22 \infty$ & 2.35 \\
\hline Ma 36 & $1 *$ & $10^{*}$ & 1.91 & 20.00 & 1.90 \\
\hline Mix 3 avg. & 1.00 & 10.00 & 1.72 & 21.00 & 2.13 \\
\hline Mx $4 a$ & 15.00 & 0.02 & 0.57 & 24.00 & 2.05 \\
\hline Mix 4b & 14.00 & 0.02 & 0.50 & $22 \infty$ & 2.01 \\
\hline Mix 4 avg. (Control, CT) & 14.50 & 0.02 & 0.54 & 23.00 & 2.03 \\
\hline Mix $5 a$ & $1 *$ & 0.05 & 3.05 & 48.00 & 1.80 \\
\hline$M \times 50$ & $1^{*}$ & 0.08 & 3.35 & 49.00 & 228 \\
\hline Mix 5 avg. & 1.00 & 0.07 & 3.20 & 48.50 & 2.04 \\
\hline$M \dot{x} 6 a$ & 3.00 & 0.07 & 288 & 50.00 & 2.13 \\
\hline$M i x \sigma b$ & $1 *$ & 0.08 & 3.09 & 50.00 & 2.62 \\
\hline Mix 6 avg. & 2.00 & 0.08 & 2.99 & 50.00 & 2.38 \\
\hline $\mathrm{M} \times \mathrm{7a}$ & 4.00 & 0.17 & 7.38 & $60 . \infty$ & 1.25 \\
\hline$M \propto 7 b$ & 3.00 & 0.14 & 6.28 & $61 . \infty$ & 1.41 \\
\hline MIX 7 avg. & 3.50 & 0.16 & 6.83 & 60.50 & 1.33 \\
\hline Mix $8 a$ & $1 *$ & 0.07 & 3.28 & 53.00 & 1.64 \\
\hline Mix $8 b$ & $1 *$ & 0.08 & 2.90 & 5200 & 1.97 \\
\hline MIX 8 avg. & 1.00 & 0.08 & 3.09 & 5250 & 1.81 \\
\hline Mix $S a$ & $1 *$ & $10^{\circ}$ & 1.64 & 29.00 & 2.20 \\
\hline$M \times 96$ & $1 *$ & $10^{\circ}$ & 0.87 & 21.00 & 209 \\
\hline MIX 9 avg. & 1.00 & 10.00 & 1.26 & 25.00 & 2.15 \\
\hline Avg al mix except CT & 1.44 & 5.05 & 266 & 40.06 & 1.94 \\
\hline Avg for CT & 14.50 & 0.02 & 0.54 & 23.00 & 203 \\
\hline Percent ofference & $-908.70 \%$ & $99.60 \%$ & $79.88 \%$ & $4259 \%$ & $-4.84 \%$ \\
\hline
\end{tabular}

Table 4 


\section{Summary}

In most cases the variation in chemical characteristics between the ash mixtures and the soil controls were not statistically significant. The elements or chemical parameters where the control concentrations were significantly higher than some of the ash mixtures were:

1) The soil concentration levels of iron averaged 75, 000 PPM, while most of the ash iron mixtures were in the range of $14,000-50,000$ PPM.

2) The nitrate/nitrite levels averaged 14.58 in the soil control but were at non-detect levels in the ash mixtures. On the other hand, the total nitrogen levels in the mixtures with sewage sludge were higher than the total nitrogen levels in the native soil.

The elements or chemical parameters where there were large differences between the H-POWER ash concentrations and the MC ash levels were:

1) The H-POWER bottom ash arsenic concentrations which had non-detect concentrations were lower than MC's bottom ash which averaged 10 PPM.

2) The H-POWER cadmium concentrations in the bottom ash were at non-detect levels (0.05 PPM) while MC averaged 7 PPM.

3) The H-POWER zinc concentrations averaged lower at 2,000 PPM than MC's ( 7,000 PPM) in the fly ash, but was higher at 12,000 PPM in the bottom ash than MC which averaged 1,300 PPM.

\section{Conclusions}

The chemical elemental analysis indicates most ash mixtures are not significantly different in metal concentrations from the soil control except for lead and zinc, where modest elevations are present. The natural pozzolanic reactions that will likely occur in the field with the addition of water, will tend to bind these and other metals to a crystalline matrix. This will cause the daily cover to be more stable, firm and less susceptible to erosion. Also the in situ chemical reactions will help make the metals less friable for dusting, more impermeable, and thus, less leachable. 
The chemical analyses of the ash/sewage sludge mixtures indicate that the high $\mathrm{pH}$ and relatively low sulfur, carbon, and nitrogen levels will make these mixtures relatively poor material for landfill final top cover by themselves. However, such mixtures are shown to be ideal for use as the infiltration and erosion layers of final landfill cover.

Ash Mixture Contents

\begin{tabular}{|c|l|}
\hline Mixture & \multicolumn{1}{|c|}{ Mixture Contents } \\
\hline 1 & H-POWER Fly Ash \\
\hline 2 & H-POWER Bottom Ash \\
\hline 3 & H-POWER Combined Ash (1) \\
\hline 4 & Landfill Soil (control) \\
\hline 5 & $\begin{array}{l}\text { 3 Parts H-POWER Fly Ash to 1 Part } \\
\text { Sewage Sludge }\end{array}$ \\
\hline 6 & $\begin{array}{l}\text { I Part H-POWER Fly Ash, 1 Part AES } \\
\text { Coal Ash, 1 Part Sewage Sludge }\end{array}$ \\
\hline 7 & $\begin{array}{l}\text { 1 Part H-POWER Fly Ash to 1 Part } \\
\text { Sewage Sludge }\end{array}$ \\
\hline 8 & $\begin{array}{l}\text { 2 Parts H-POWER Fly Ash, 2 Parts } \\
\text { Sewage Sludge, 1 Part Hydrated Lime }\end{array}$ \\
\hline 9 & $\begin{array}{l}\text { 1 Part H-POWER Combined Ash to 1 } \\
\text { Part Waipahu Ash }\end{array}$ \\
\hline
\end{tabular}

Table 9 


\section{Laboratory Report}

Zlient: $\quad$ NREL/ H-Power Ash Utilization 23 Rexinger Lane

Avon, CT 06001

Attention: Michael Hartman
Page: $\quad 1$ of 6

ELP Project No.: $\quad 5347$

Report Date: 10-Feb-94

Sample Description: Samples from H-Power.

Date Collected: see below Sample Matrix: ash

Date Received: 12-Nov-93

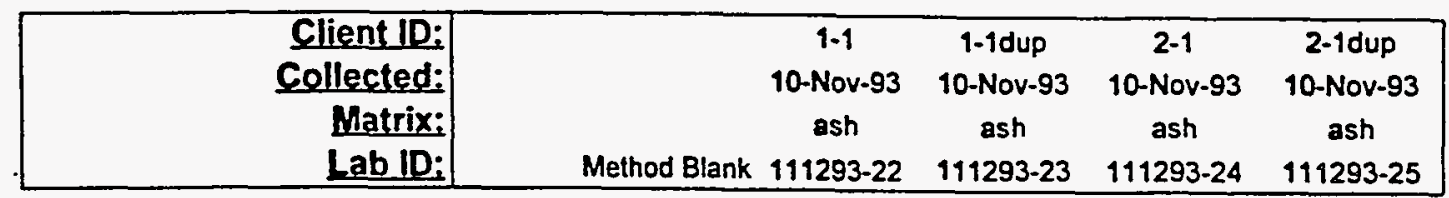

\begin{tabular}{|c|c|c|c|c|c|c|c|c|c|}
\hline Date & $\begin{array}{l}\text { Analysis } \\
\text { Iotal Metals in soil }\end{array}$ & Method & Units & MRL & Results & Results & Results & Results & Results \\
\hline 22-Dec-93 & Metals Digestion (ICP) & EPA 3050M & & & & & & & \\
\hline 11-Jan-94 & Aluminum & EPA 6010 & $\mathrm{mg} / \mathrm{Kg}(\mathrm{ppm})$ & 20 & ND & 10,223 & 5,536 & 48,985 & 61.120 \\
\hline 07-Jan-94 & Arsenic & EPA 6010 & $\mathrm{mg} / \mathrm{Kg}(\mathrm{ppm})$ & 50 & ND & 76 & 75 & ND & ND \\
\hline 17-Jan-94 & Barium & EPA 6010 & $\mathrm{mg} / \mathrm{Kg}(\mathrm{ppm})$ & 10 & ND & 310 & 310 & 570 & 810 \\
\hline 17-Jan-94 & Cadmium & EPA 6010 & $\mathrm{mg} / \mathrm{Kg}(\mathrm{ppm})$ & 5 & ND & 26 & 27 & ND & ND \\
\hline 11-Jan-94 & Calcium & EPA 6010 & $\mathrm{mg} / \mathrm{Kg}(\mathrm{ppm})$ & 20 & ND & 107,248 & 99,844 & 109,122 & 101,082 \\
\hline 07-Jan-94 & Chromium & EPA 6010 & $\mathrm{mg} / \mathrm{Kg}(\mathrm{ppm})$ & 5 & ND & 116 & 120 & 160 & 214 \\
\hline 07-Jan-94 & Copper & EPA 6010 & $\mathrm{mg} / \mathrm{Kg}(\mathrm{ppm})$ & 10 & ND & 241 & 260 & 3,800 & 940 \\
\hline 11-Jan-94 & Iron & EPA 6010 & $\mathrm{mg} / \mathrm{Kg}(\mathrm{ppm})$ & 90 & ND & 14,953 & 17,512 & 27,401 & 50,101 \\
\hline 11-Jan-94 & Lead & EPA 6010 & $m g / K g(p p m)$ & 20 & ND & 1,707 & 9,861 & 828 & 14,748 \\
\hline 14-Feb-94 & Mercury & EPA 7471 & $\mathrm{mg} / \mathrm{Kg}(\mathrm{ppm})$ & 0.2 & ND & 10 & 14 & 12 & 5.3 \\
\hline 07-Jan-94 & Polassium & EPA 6010 & $\mathrm{mg} / \mathrm{Kg}(\mathrm{ppm})$ & 20 & ND & 8,640 & 8,649 & 7,950 & 7,565 \\
\hline 07-Jan-94 & Selenium & EPA 6010 & $\mathrm{mg} / \mathrm{Kg}(\mathrm{ppm})$ & 50 & ND & ND & ND & ND & ND \\
\hline 25-Jan-94 & Silicon* & EPA 6010 & $\mathrm{mg} / \mathrm{Kg}(\mathrm{ppm})$ & 10,000 & ND & 62,986 & 62,747 & 158,738 & 169,682 \\
\hline 07-Jan-94 & Silver & EPA 6010 & $\mathrm{mg} / \mathrm{Kg}(\mathrm{ppm})$ & 5 & ND & 10 & 7 & ND & ND \\
\hline 07-Jan-94 & $\begin{array}{l}\text { Zinc } \\
\text { Wet Chemistry }\end{array}$ & EPA 6010 & $\mathrm{mg} / \mathrm{Kg}(\mathrm{ppm})$ & 10 & ND & 2,106 & 1,700 & 23,800 & 2,039 \\
\hline 22-Nov-93 & $\mathrm{pH}$ & EPA 9045 & units & 0.01 & NA & 11.69 & 11.64 & 11.91 & 11.76 \\
\hline 19-Nov-93 & Chloride & EPA 325.3 & $\%$ & 0.001 & ND & 2.9 & 2.6 & 0.26 & 0.053 \\
\hline 19-Nov-93 & Sulfate & EPA 375.4 & $\%$ & 0.005 & ND & 0.043 & 0.015 & 0.0018 & 0.0024 \\
\hline 22-Dec-93 & Nitrogen, Nitrate+Nitrite $(\mathrm{N})^{* *}$ & EPA 353.3 & $\mathrm{mg} / \mathrm{Kg}(\mathrm{ppm})$ & 2 & ND & $<2$ & $<2$ & $<2$ & $<2$ \\
\hline 22-Dec-93 & Nitrogen, Total Kjeldahl $(\mathbf{N})^{-\infty}$ & EPA 351.3 & $\mathrm{mg} / \mathrm{Kg}$ (ppm) & 20 & ND & NR & NR & NR & $N R$ \\
\hline 22-Nov-93 & Moisture & CLP ILM 2.0 & percent & NA & NA & 12 & 41 & 23 & 20 \\
\hline 05-Jan-94 & Density, Apparent & $\cdots$ & $g / c c$ & NA & NA & 0.937 & 0.967 & 0.916 & 1.32 \\
\hline 05-Jan-94 & Density. True & $\cdots$ & $g / c c$ & NA & NA & 1.79 & 1.69 & 1.76 & 2.10 \\
\hline 22-Nov-93 & Carbon"* & ASTM D5291M & percent & 0.1 & NA & 1.63 & 1.75 & 0.46 & 0.55 \\
\hline
\end{tabular}

- Perkin Elmer "Analytical Methods for AAS" Jan 1982

- Analysis performed by Huffman Laboratories, Inc.

- Methods of Soil Analysis, American Society of Agronomy, Part 1, 1982
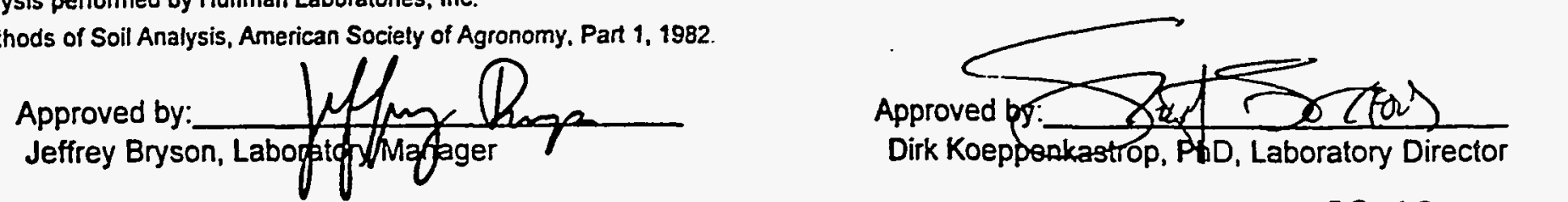

A3-13 
Page:

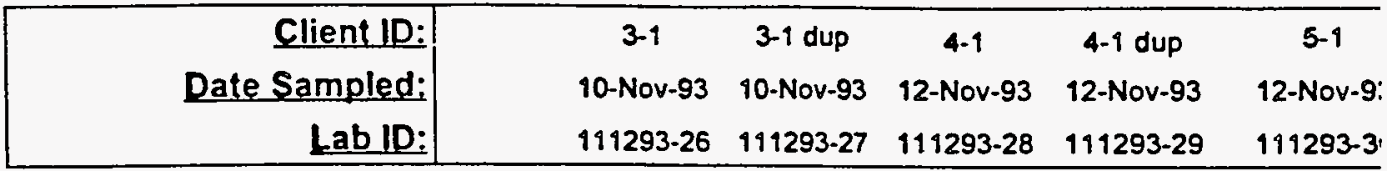

\begin{tabular}{|c|c|c|c|c|c|c|c|c|c|}
\hline Date & $\begin{array}{l}\text { Analysis } \\
\text { Total Metals in soil }\end{array}$ & Method & Units & MRL & Results & Results & Results & Results & Results \\
\hline 22-Jan-94 & Metals Digestion (ICP) & EPA $3050 \mathrm{M}$ & & & & & & & \\
\hline 41-Jan-94 & Aluminum & EPA 6010 & $\mathrm{mg} / \mathrm{Kg}(\mathrm{ppm})$ & 20 & 46,341 & 47,469 & 55,563 & 50,755 & 26,877 \\
\hline 07-Jan-94 & Arsenic & EPA 6010 & $\mathrm{mg} / \mathrm{Kg}(\mathrm{ppm})$ & 50 & ND & 87 & ND & ND & 73 \\
\hline 07-Jan-94 & Barium & EPA 6010 & $\mathrm{mg} / \mathrm{Kg}(\mathrm{ppm})$ & 10 & ND & 537 & 184 & 73 & 405 \\
\hline 07-Jan-94 & Cadmium & EPA 6010 & $\mathrm{mg} / \mathrm{Kg}(\mathrm{ppm})$ & 5 & 11 & 105 & ND & ND & 23 \\
\hline 91-Jan-94 & Calcium & EPA 6010 & $\mathrm{mg} / \mathrm{Kg}(\mathrm{ppm})$ & 20 & 131,156 & 118,166 & 24,145 & 36,121 & 112,753 \\
\hline 07-Jan-94 & Chromium & EPA 6010 & $\mathrm{mg} / \mathrm{Kg}(\mathrm{ppm})$ & 5 & 206 & 181 & 250 & 310 & $\$ 13$ \\
\hline 07-Jan-94 & Copper & EPA 6010 & $\mathrm{mg} / \mathrm{Kg}(\mathrm{ppm})$ & 10 & 5,126 & 659 & 95 & 102 & 245 \\
\hline 19-Jan-94 & Iron & EPA 6010 & $\mathrm{mg} / \mathrm{Kg}(\mathrm{ppm})$ & 10 & 25,380 & 24,782 & 64,235 & 85,756 & 13,874 \\
\hline 14-Jan-94 & Lead & EPA 6010 & $\mathrm{mg} / \mathrm{Kg}(\mathrm{ppm})$ & 20 & 3,172 & 15,809 & 48 & 26 & 1,437 \\
\hline 94-Dec-93 & Mercury & EPA 7471 & $\mathrm{mg} / \mathrm{Kg}(\mathrm{ppm})$ & 0.2 & 8.2 & 7.7 & 5.2 & 12 & 14 \\
\hline 02-Jan-94 & Potassium & EPA 6010 & $\mathrm{mg} / \mathrm{Kg}(\mathrm{ppm})$ & 20 & 10,800 & 12,231 & 1,393 & 1,032 & 8,457 \\
\hline 02-Jan-94 & Selenium & EPA 6010 & $\mathrm{mg} / \mathrm{Kg}(\mathrm{ppm})$ & 50 & ND & ND & ND & ND & ND \\
\hline 25-Jan-94 & Silicon* & EPA 6010 & $\mathrm{mg} / \mathrm{Kg}(\mathrm{ppm})$ & 10,000 & 116,705 & 113,747 & 174,885 & 273,319 & 56,494 \\
\hline 07-Jan-94 & Silver & EPA 6010 & $\mathrm{mg} / \mathrm{Kg}$ (ppm) & 5 & 8 & 16 & ND & ND & ND \\
\hline \multirow[t]{2}{*}{ 07-Jan-94 } & Zinc & EPA 6010 & $m g / K g(p p m)$ & 10 & 2,230 & 4,062 & 116 & 123 & 1,992 \\
\hline & WetChemistry & & & & & & & & \\
\hline 22-Nov-93 & pH & EPA 9045 & units & 0.01 & 11.88 & 11.85 & 7.96 & 8.33 & 91.67 \\
\hline 19-Nov-93 & Chloride & EPA 325.3 & $\%$ & 0.0001 & 1.0 & 1.37 & 0.053 & 0.11 & 2.5 \\
\hline 19-Nov-93 & Sulfate & EPA 375.4 & $\%$ & 0.005 & 0.0065 & 0.011 & 0.011 & 0.012 & 0.026 \\
\hline 22-Dec-93 & Nitrogen, Nitrate+Nitrite $(\mathbf{N})^{\infty}$ & EPA 353.3 & $\mathrm{mg} / \mathrm{Kg}(\mathrm{ppm})$ & 2 & $<2$ & $<2$ & 15 & 14 & $<2$ \\
\hline 22-Dec-93 & Nitrogen, Total Kjeldahl $(\mathbf{N})^{\infty}$ & EPA 351.3 & $\mathrm{mg} / \mathrm{Kg}(\mathrm{ppm})$ & 200 & NR & NR & 0.02 & 0.02 & 0.05 \\
\hline 22-Nov-93 & Moisture & CLP ILM 2.0 & percent & NA & 22 & 20 & 24 & 22 & $\$ 8$ \\
\hline 05-Jan-94 & Density, Apparent & $\infty$ & $g / c c$ & NA & 0.991 & 1.37 & 1.29 & 1.40 & 0.979 \\
\hline 05-Jan-94 & Density, True & $\infty$ & $g / c c$ & NA & 2.35 & 1.90 & 2.05 & 2.01 & 1.80 \\
\hline 22-Nov-93 & Carbon"* & ASTM D5291M & percent & 0.1 & 1.53 & 1.91 & 0.57 & 0.5 & 3.05 \\
\hline
\end{tabular}

- Perkin Elmer "Analytical Methods for AAS" Jan 1982

- Analysis performed by Huffman Laboratories. Inc.

-. Methods of Soil Analysis. American Society of Agronomy, Part 1, 1982.

Approved by:

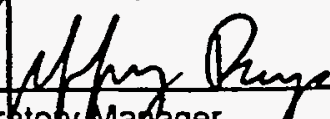

Jeffrey Bryson, Laborptopy Mareger

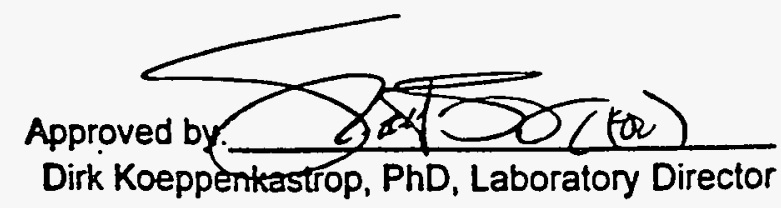

A3-14 
Page:

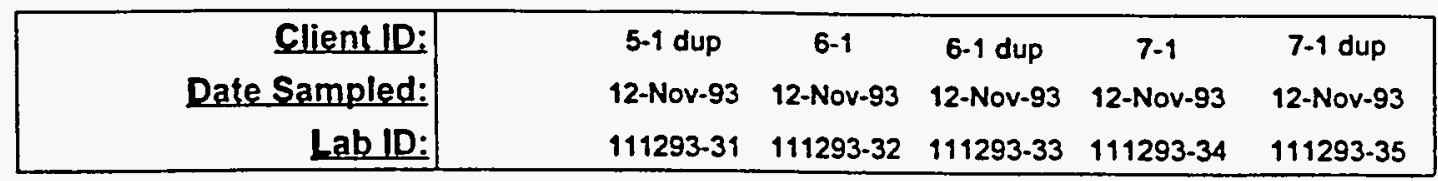

\begin{tabular}{|c|c|c|c|c|c|c|c|c|c|}
\hline Date & $\begin{array}{l}\text { Analysis } \\
\text { Total Metals in soil }\end{array}$ & Method & Units & MRL & Results & Results & Results & Results & Results \\
\hline 22-Jan-94 & Metals Digestion (ICP) & EPA 3050M & & & & & & & \\
\hline 14-Jan-94 & Aluminum & EPA 6010 & $\mathrm{mg} / \mathrm{Kg}(\mathrm{ppm})$ & 20 & 26,758 & 41,040 & 23,197 & 16,835 & 19,585 \\
\hline 07-Jan-94 & Arsenic & EPA 6010 & $\mathrm{mg} / \mathrm{Kg}(\mathrm{ppm})$ & 50 & 74 & ND & ND & ND & ND \\
\hline 07-Jan-94 & Barium & EPA 6010 & $\mathrm{mg} / \mathrm{Kg}(\mathrm{ppm})$ & 10 & 360 & 238 & 220 & 233 & 270 \\
\hline 07-Jan-94 & Cadmium & EPA 6010 & $\mathrm{mg} / \mathrm{Kg}(\mathrm{ppm})$ & 5 & 24 & 7 & 6 & 16 & 15 \\
\hline 14-Jan-94 & Calcium & EPA 6010 & $\mathrm{mg} / \mathrm{Kg}$ (ppm) & 20 & 123,589 & 81,646 & 75,629 & 101,861 & 94,160 \\
\hline 07-Jan-94 & Chromium & EPA 6010 & $\mathrm{mg} / \mathrm{Kg}$ (ppm) & 5 & 110 & 65 & 46 & 83 & 84 \\
\hline 07-Jan-94 & Copper & EPA 6010 & $\mathrm{mg} / \mathrm{Kg}(\mathrm{ppm})$ & 10 & 275 & 78 & 80 & 199 & 220 \\
\hline 14-Jan-94 & Iron & EPA 6010 & $\mathrm{mg} / \mathrm{Kg}(\mathrm{ppm})$ & 10 & 15,150 & 16,815 & 15,261 & 100,740 & 13,088 \\
\hline 14-Jan-94 & Lead & EPA 6010 & $\mathrm{mg} / \mathrm{Kg}(\mathrm{ppm})$ & 20 & 1,582 & 669 & 160 & 1,150 & 840 \\
\hline 14-Dec-93 & Mercuny & EPA 7471 & $\mathrm{mg} / \mathrm{Kg}(\mathrm{ppm})$ & 0.2 & 13 & 12 & 11 & 12 & 10 \\
\hline 07-Jan-94 & Potassium & EPA 6010 & $\mathrm{mg} / \mathrm{kg}(\mathrm{ppm})$ & 20 & 8,574 & 6,066 & 5,770 & 6,811 & 6,111 \\
\hline 14-Jan-94 & Selenium & EPA 6010 & $\mathrm{mg} / \mathrm{Kg}(\mathrm{ppm})$ & $\mathbf{5 0}$ & ND & ND & ND & ND & ND \\
\hline 25-Jan-94 & Silicon* & EPA 6010 & $\mathrm{mg} / \mathrm{kg}(\mathrm{ppm})$ & 10.000 & 57,485 & 62,943 & 52,684 & 46,204 & 43,523 \\
\hline 07-Jan-94 & Silver & EPA 6010 & $m g / k g(p p m)$ & 5 & 9 & 6 & 7 & 11 & 14 \\
\hline 07-Jan-94 & $\begin{array}{l}\text { Zinc } \\
\text { WetChemistry }\end{array}$ & EPA 6010 & $\mathrm{mg} / \mathrm{kg}(\mathrm{ppm})$ & 10 & 2,119 & 735 & 640 & 1,538 & 1,468 \\
\hline 22-Nov-93 & pH & EPA 9045 & units & 0.01 & 11.66 & 11.94 & 11.85 & 11.81 & 11.85 \\
\hline 19-Nov-93 & Chloride & EPA 325.3 & $\%$ & 0.0001 & 1.19 & 0.37 & 0.77 & 1.8 & 1.8 \\
\hline 19-Nov-93 & Sulfate & EPA 375.4 & $\%$ & 0.005 & 0.026 & 0.12 & 0.12 & 0.023 & 0.038 \\
\hline 22-Dec-93 & Nitrogen, Nitrate+Nitrite $(\mathrm{N})^{-\infty}$ & EPA 353.3 & $\mathrm{mg} / \mathrm{kg}(\mathrm{ppm})$ & 2 & $<2$ & 3 & $<2$ & 4 & 3 \\
\hline 22-Dec-93 & Nitrogen, Total Kjeldahl (N)"• & EPA 351.3 & $\mathrm{mg} / \mathrm{Kg}(\mathrm{ppm})$ & 20 & 0.08 & 0.07 & 0.08 & 0.17 & 0.14 \\
\hline 22-Nov-94 & Moisture & CLP ILM 2.0 & percent & NA & 49 & 50 & 50 & 60 & 61 \\
\hline 05-Jan-94 & Density, Apparent & $\infty$ & $q \propto c$ & NA & 1.15 & 0.82 & 0.961 & 1.13 & 0.974 \\
\hline 05-Jan-94 & Density. True & $\infty$ & $g / c c$ & NA & 2.28 & 2.13 & 2.62 & 1.25 & 1.41 \\
\hline 22-Nov-93 & Carbon"* & ASTM D5291M & percent & 0.1 & 3.35 & 2.88 & 3.09 & 7.38 & 6.28 \\
\hline
\end{tabular}

- Perkin Elmer "Analytical Methods for AAS" Jan 1982

- Analysis performed by Huffman Laboratories, Inc.

-. Methods of Soil Analysis, American Society of Agronomy, Part 1, 1982.
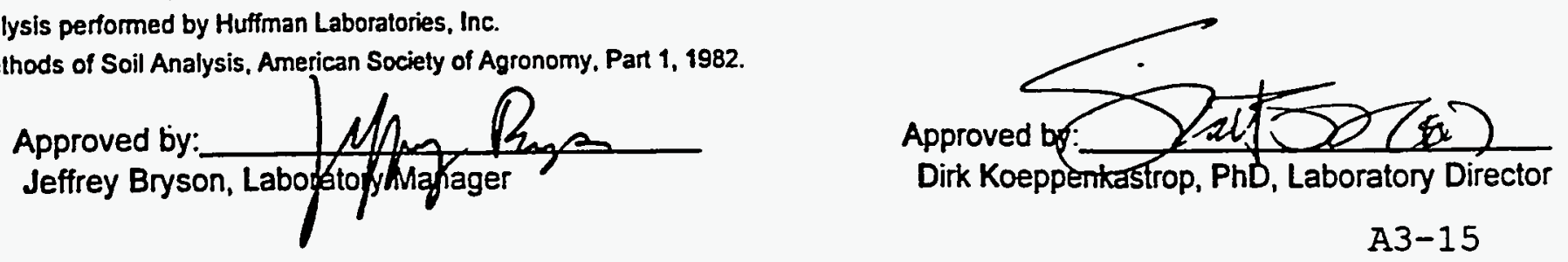


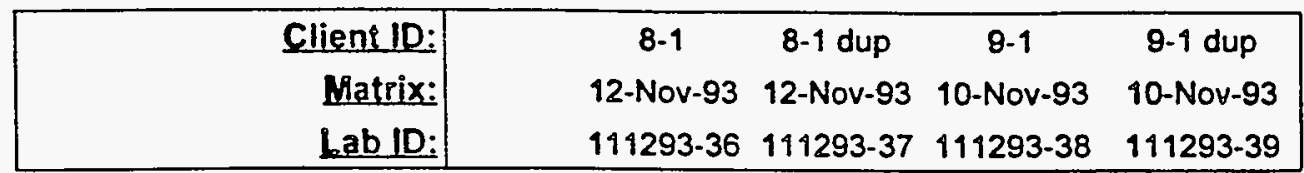

\begin{tabular}{|c|c|c|c|c|c|c|c|c|}
\hline Date & $\begin{array}{l}\text { Analysis } \\
\text { Total Metals in soil }\end{array}$ & Methed & Units & MRL & Resulis & Results & Results & Results \\
\hline 22-Jan-94 & Metals Digestion (ICP) & EPA 3050M & & & & & & \\
\hline 14-Jan-94 & Aluminum & EPA 6010 & $\mathrm{mg} / \mathrm{Kg}$ (ppm) & 20 & 10,485 & 10,303 & 49,099 & 50,794 \\
\hline 07-Jan-94 & Arsenic & EPA 6010 & $\mathrm{mg} / \mathrm{Kg}(\mathrm{ppm})$ & 50 & ND & ND & 70 & ND \\
\hline 07-Jan-94 & Barium & EPA 6010 & $\mathrm{mg} / \mathrm{Kg}(\mathrm{ppm})$ & 10 & 139 & $\$ 19$ & 391 & 303 \\
\hline 07-Jan-94 & Cadmium & EPA 6010 & $\mathrm{mg} / \mathrm{Kg}(\mathrm{ppm})$ & 5 & 8 & 8 & 25 & 20 \\
\hline 14-Jan-94 & Calcium & EPA 6010 & $\mathrm{mg} / \mathrm{Kg}(\mathrm{ppm})$ & 20 & 143,000 & 954,802 & 84,776 & 79,435 \\
\hline 07-Jan-94 & Chromium & EPA 6010 & $\mathrm{mg} / \mathrm{Kg}(\mathrm{ppm})$ & 5 & 39 & 35 & 202 & 214 \\
\hline 07-Jan-94 & Copper & EPA 6010 & $\mathrm{mg} / \mathrm{Kg}(\mathrm{ppm})$ & 10 & 98 & 76 & 464 & 429 \\
\hline 14-Jan-94 & Iron & EPA 6010 & $\mathrm{mg} / \mathrm{Kg}(\mathrm{ppm})$ & 80 & 4,742 & 4,833 & 60,132 & 104,118 \\
\hline 14-Jan-94 & Lead & EPA 6010 & $\mathrm{mg} / \mathrm{Kg}(\mathrm{ppm})$ & 20 & $\$ 47$ & 348 & 919 & 606 \\
\hline 14-Dec-93 & Mercury & EPA 7471 & $\mathrm{mg} / \mathrm{Kg}(\mathrm{ppm})$ & 0.2 & 10 & 43 & 5 & 11 \\
\hline 07-Jan-94 & Potassium & EPA 6010 & $\mathrm{mg} / \mathrm{Kg}(\mathrm{ppm})$ & 20 & 3,511 & 3,298 & 9,716 & 7,717 \\
\hline 14-Jan-94 & Selenium & EPA 6010 & $m g / K g(p p m)$ & 50 & ND & ND & ND & ND \\
\hline 25-Jan-94 & Silicon* & EPA 6010 & $\mathrm{mg} / \mathrm{Kg}(\mathrm{ppm})$ & 90.000 & 19,570 & 17,333 & 90,357 & 127,880 \\
\hline 07-Jan-94 & Silver & EPA 6010 & $\mathrm{mg} / \mathrm{Kg}(\mathrm{ppm})$ & 5 & 7 & 8 & 6 & 5 \\
\hline 07-Jan-94 & $\begin{array}{l}\text { Zine } \\
\text { Wet Chemistry }\end{array}$ & EPA 6010 & $\mathrm{mg} / \mathrm{Kg}(\mathrm{ppm})$ & 10 & 796 & 675 & 3,032 & 3,293 \\
\hline 22-Nov-93 & $\mathrm{pH}$ & EPA 9045 & units & 0.09 & $\$ 1.92$ & 11.92 & 11.94 & 14.96 \\
\hline 19-Nov-93 & Chloride & EPA 325.3 & $\%$ & 0.0001 & 0.87 & 0.98 & 0.61 & 0.58 \\
\hline 19-Jan-93 & Sulfate & EPA 375.4 & $\%$ & 0.005 & 0.010 & 0.0087 & 0.0071 & 0.0086 \\
\hline 22-Dec-93 & Nitrogen, Nitrate+Nitrite $(N)^{* *}$ & EPA 353.3 & $\mathrm{mg} / \mathrm{Kg}(\mathrm{ppm})$ & 2 & $<2$ & $<2$ & $<2$ & $<2$ \\
\hline 22-Dec-93 & Nitrogen, Total Kjeldahl (N)"• & EPA 351.3 & $\mathrm{mg} / \mathrm{Kg}(\mathrm{ppm})$ & 20 & 0.07 & 0.08 & NR & $N R$ \\
\hline 22-Nov-93 & Moisture & CLP ILM 2.0 & percent & NA & 53 & 52 & 29 & 21 \\
\hline 05-Jan-94 & Density, Apparent & $\infty$ & $g / c$ & NA & 1.36 & 1.41 & 9.34 & 0.850 \\
\hline 05-Jan-94 & Density, True & $\cdots$ & $g / c c$ & NA & 1.64 & 1.97 & 2.2 & 2.09 \\
\hline 22-Nov-93 & Carbon* & ASTM D5291M & percent & 0.9 & 3.28 & 2.90 & 1.64 & 0.87 \\
\hline
\end{tabular}

- Perkin Elmer "Analytical Methods for AAS" Jan 1982

- Analysis performed by Huffman Laboratories, Inc.

- Methods of Soil Analysis, American Society of Agronomy. Part 1, 1982.

Approved by:
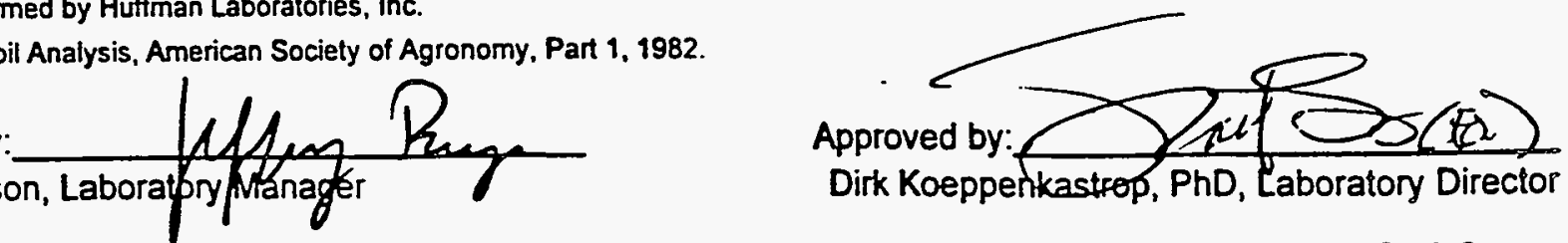


\section{Quality Control Data}

\begin{tabular}{|c|c|c|c|c|c|c|c|c|}
\hline \multicolumn{2}{|l|}{ SPIKES } & \multirow{2}{*}{$\begin{array}{r}\begin{array}{r}\text { Lab ID: } \\
\text { Units: }\end{array} \\
\text { Method }\end{array}$} & \multirow{2}{*}{$\begin{array}{c}\begin{array}{c}\text { LCS1 } \\
\% R\end{array} \\
\text { Results }\end{array}$} & \multirow{2}{*}{$\begin{array}{c}\begin{array}{c}\text { LCS2 } \\
\% R\end{array} \\
\text { Results }\end{array}$} & \multirow{2}{*}{$\begin{array}{c}\text { RPD } \\
\text { Results }\end{array}$} & \multirow{2}{*}{$\begin{array}{c}\text { MS } \\
\% R \\
\text { Results }\end{array}$} & \multirow{2}{*}{$\begin{array}{c}\text { MSD } \\
\% R \\
\text { Results }\end{array}$} & \multirow{2}{*}{$\begin{array}{c}\text { RPD } \\
\text { Results }\end{array}$} \\
\hline Lab ID & $\begin{array}{l}\text { Analysis } \\
\text { Total Metals in soil }\end{array}$ & & & & & & & \\
\hline $111293-30$ & Aluminum & EPA 6010 & 87 & 96 & 10 & $\infty$ & 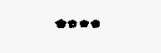 & $\infty$ \\
\hline $111293-30$ & Arsenic & EPA 6010 & 101 & 98 & 3 & 104 & 110 & 6 \\
\hline $111293-30$ & Barium & EPA 6010 & 107 & 106 & 1 & 70 & 81 & 15 \\
\hline $111293-30$ & Barium (PDS) & EPA 6010 & NA & NA & NA & 95 & 97 & 2 \\
\hline $111293-30$ & Cadmium & EPA 6010 & 99 & 93 & 6 & 97 & 100 & 3 \\
\hline $111293-30$ & Calcium & EPA 6010 & 107 & 96 & 11 & 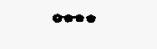 & $\infty$ & $\infty$ \\
\hline $111293-30$ & Chromium & EPA 6010 & 105 & 90 . & 15 & 99 & 86 & 14 \\
\hline $111293-30$ & Copper & EPA 6010 & 107 & 103 & 4 & 112 & 101 & 40 \\
\hline $111293-30$ & Iron & EPA 6010 & 109 & 95 & 14 & 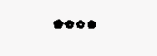 & $\cdots$ & $\infty$ \\
\hline $111293-30$ & Lead & EPA 6010 & 99 & 93 & 6 & 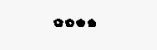 & $\infty$ & $\infty$ \\
\hline $111293-22$ & Mercury & EPA 7471 & 123 & 115 & 7 & $\infty$ & $\cdots$ & $\infty$ \\
\hline $111293-30$ & Potassium & EPA 6010 & 77 & 208 & 92 & 101 & 95 & 6 \\
\hline $111293-30$ & Selenium & EPA 6010 & 101 & 98 & 3 & 102 & 90 & 13 \\
\hline $111293-30$ & Silicon* & EPA 6010 & 112 & 107 & 5 & $\cdots$ & $\infty$ & $\infty$ \\
\hline $111293-30$ & Silicon (PDS) & EPA 6010 & NA & NA & NA & 108 & 116 & 7 \\
\hline $111293-30$ & Silver & EPA 6010 & 32 & 63 & 65 & 101 & 98 & 3 \\
\hline $111293-30$ & Silver (PDS) & EPA 6010 & NA & NA & NA & 102 & 96 & 6 \\
\hline $111293-30$ & Zinc & EPA 6010 & 104 & 97 & 7 & 21 & 73 & 111 \\
\hline \multirow[t]{2}{*}{$111293-30$} & Zinc (PDS) & EPA 6010 & NA & NA & NA & 96 & 113 & 16 \\
\hline & Wet Chemistry in soil & & & & & & & \\
\hline $111293-23$ & Chloride & EPA 325.3 & 109 & 102 & 1 & 106 & 111 & 5 \\
\hline $111293-35$ & Sulfate & EPA 375.4 & 92 & 94 & 2 & 105 & 103 & 2 \\
\hline $120193-19$ & Sulfate & EPA 375.4 & 100 & 901 & 1 & 103 & 106 & 3 \\
\hline \multicolumn{2}{|c|}{ DUPLICATES } & $\begin{array}{l}\text { Lab ID: } \\
\text { Units: }\end{array}$ & \multicolumn{3}{|c|}{ are mg/ unless othènwise noted } & OS & $\mathrm{D}$ & $\begin{array}{c}\text { RPD } \\
\text { percent }\end{array}$ \\
\hline \multirow[t]{2}{*}{ Lab ID } & Analysis & Method & & & & Results & Results & Results \\
\hline & $\begin{array}{l}\text { Wet Chemistry in soil } \\
\text { Moisture }\end{array}$ & percent & & & & 21 & 14 & 40 \\
\hline
\end{tabular}

- Perkin Elmer "Analytical Methods for AAS" Jan 1982

-.. Native analyte greater than 4 times the spike added, therefore recovery not calculable.
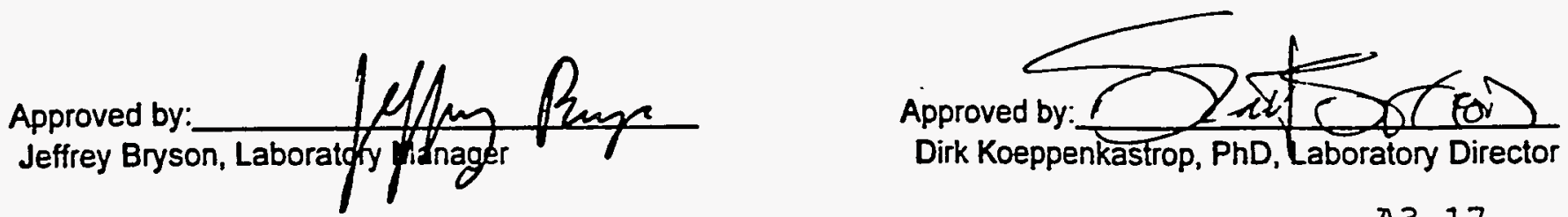


\section{Definitions}

$\begin{array}{ll}\text { D } & \text { Duplicate } \\ \text { LCS } & \text { Laboratory Control Sample } \\ \text { MS } & \text { Matrix Spike } \\ \text { MSD } & \text { Matrix Spike Duplicate } \\ \text { MRL } & \text { Method Reporting Limit } \\ \text { NA } & \text { Not Applicable } \\ \text { ND } & \text { Not Detected at the MRL } \\ \text { NR } & \text { Not Requested } \\ \text { OS } & \text { Original Sample } \\ \text { \%R } & \text { Percent Recovery } \\ \text { PDS } & \text { Post Digestion Spike } \\ \text { RPD } & \text { Relative Percent Difference }\end{array}$


E. H. De CARLO, Ph.D.

ANALYTICAL, ENVIRONMENTAL AND MARINE CONSULTANTS

2654 Lowrey Avenue

Honolulu, Hawaii 96822

$(808)-988-5028$

\section{December 1993}

Executive Summary:

A series of samples was recejved from Environmental Laboratory of the Pacific (ELP) to be digested by procedures that could not be feasibly performed by the client laboratory. Twenty individual samples were provided consisting of heterogenous powders/gravelly solids with variable water content. A brief summary of the visual appearance of some samples follows to provide an indication of the extent of variability amongst individual samples. Certain samples were quite wet such as 111293-32, 111293-36, and 111293-38, others quite homogenous such as 111693-21, and others quite heterogeneous such as 111293-22.

For further example the following descriptions apply to the first batch of digested samples.

111693-21 Relatively dry and homogenous sample.

111693-22 Wet and very cohesive yet heterogeneous material.

111293-22 Fine-grained material present as a wet paste. 111293-23 Wet globular, somewhat heterogenous material.

111293-24 Gravelly, heterogeneous moist material.

111293-25 Wet gravelly, heterogeneous material.

111293-26 Coarse, yet more homogenous moist material.

111293-27 Moist, coarse material with some chunks of greyish other material. This sample was quite hygroscopic and gained weight rapidly when weighed on an analytical balance.

111293-28 Very smooth, clayey rather homogenous brown material.

The samples were taken "as is" for the digestion procedure which was conducted as follows:

A mass of "as is" sample was weighed (to the nearest $0.1 \mathrm{mg}$ ) into 120 psi-rated CEM Teflon microwave digestion vessels. To each sample was added $5 \mathrm{~mL}$ of $30 \% \mathrm{H}_{2} \mathrm{O}_{2}$ to oxidize organic matter. After the subsidence of the reaction with $\mathrm{H}_{2} \mathrm{O}_{2}$, as evidenced by a lack of further gas evolution, a mixture of $15 \mathrm{~mL} 2: 1$ concentrated $\mathrm{HNO}_{3}: \mathrm{HCl}$ and $7 \mathrm{~mL}$ concentrated HF was added and the vessels sealed. Vessels were then placed in a carousel, connected via vent tubes to a $\mathrm{NaHCO}_{3}$ neutralizing bath (in case of venting of acid fumes) then placed in a microwave oven. Digestion was performed as a series of repetitive 
low power runs ranging from 208 power for 30 minutes (per carousel of 12 vessels) to $40 \%$ power for 30 minutes. After digestion appeared to not proceed further, vessels were cooled, opened and 50 $\mathrm{mL}$ of $0.5 \mathrm{M} \mathrm{H}_{3} \mathrm{BO}_{3}$ added. The vessels were resealed and placed in the microwave oven for a further run at $50 \%$ power for 60 minutes. It should be noted that samples were cooled in between runs whenever the pressure in the vessels appeared excessive or a slight extent of sample venting was observed. This procedure was

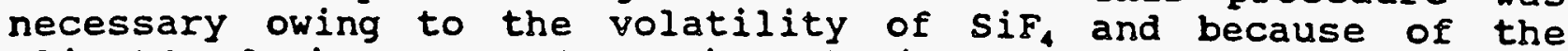
client's desire to perform $s i$ analysis. In general samples were very difficult to digest and residues remained even after extensive heating in the microwave oven. -

Several procedural blanks were prepared by only adding acids and carrying the vessels through the procedure. Two blanks were also spiked prior to digestion as requested by ELP personnel. Three actual samples were also spiked prior to digestion as requested. Spikes consisted of the addition of $1 \mathrm{~mL} 1000 \mathrm{ppm} \mathrm{Si,} 2 \mathrm{~mL}$ QC23 100 ppm multi-element spike, and $100 \mu \mathrm{L} 1000 \mathrm{ppm} \mathrm{Ag.}$

Because residues remained in most samples after the digestion procedure a second digestion was performed with further acid additions. This procedure was employed only for the first batch of samples (111653 series and 111293-22 through 28). These samples were then filtered at the end of the procedure owing to the presence of some residues. Final masses of the first batch of samples is near $200 \mathrm{~g}$. Residues were retained and their weight recorded after air-drying. Individual sample masses and final solution masses are reported on labels on the individual sample solution bottles and are not repeated here. The second and third batches of samples were only carried through a single digestion procedure, hence, their final masses are near $100 \mathrm{~g}$. Because smaller masses of original sample were used in the second and third digestion procedures, residues tended to be much smaller.

special notes regarding individual samples follow.

Samples 111693-21, 111293-22, 111293-23, 111293-27, 111293-31, $111293-36,111293-37$ all reacted strongly with $\mathrm{H}_{2} \mathrm{O}_{2}$.

Samples 111693-22, 111293-24, 111293,25 reacted vigorously with aqua-regia, whereas samples $111293-30,31,32,33,34$ and 35 reacted well with aqua-regia but not as vigorously as the previous batch.

One sample (111293-29) was digested in duplicate because the first replicate was observed to contain a large chunk (relative to overall sample mass) of what appeared to be basaltic material after the $\mathrm{H}_{2} \mathrm{O}_{2}$ step, hence the second replicate of this sample, which did not contain such a chunk, is more likely to be representative.

Some samples were obviously organic-rich as evidenced by yellow- 
orange coloration in the final digested solutions. Several of these subsequently developed a precipitate in the solution although they were completely clear immediately after filtration. It is suspected that these precipitates may represent insoluble organic acids.

The following is a summary of residue masses and their appearance:

111693-21:
$111693-21$ spike:
$111693-22:$
$111293-22:$
$111293-23:$
$111293-24:$
$111293-25:$
$111293-26:$
$111293-27:$
$111293-28:$
$111293-29:$
$111293-30:$
$111293-30$ Spike:
$111293-31:$
$111293-32:$
$111293-33:$
$111293-34:$
$111293-35:$
$111293-36:$
$111293-36$ Spike:
$111293-29$ Repl:
$111293-37:$
$111293-38:$
$111293-29$

111693-21:

111693-22:

111293-22:

111293-23:

111.293-24:

111293-25:

111293-26:

111293-27:

111293-28:

111293-29:

111293-30:

111293-30spike:

111293-31:

111293-32:

111293-33:

111293-34:

$112293-35$

111293-36Spike:

111293-29Repl:

111293-38:

111293-29
$11 \mathrm{mg} \mathrm{black}$
$11 \mathrm{mg}$ black
$131 \mathrm{mg}$ grey
$87 \mathrm{mg}$ dark grey
$101 \mathrm{mg}$ dark grey
$78 \mathrm{mg}$ grey and white
$106 \mathrm{mg}$ grey and white
$55 \mathrm{mg}$ black and white
$84 \mathrm{mg}$ black and white
no residue
$57 \mathrm{mg}$ grey/white (chunk)
$10 \mathrm{mg}$ black
$20 \mathrm{mg}$ dark grey/black
$12 \mathrm{mg}$ black
7 mg black
$12 \mathrm{mg}$ grey/black
$13 \mathrm{mg}$ grey/black
$7 \mathrm{mg}$ grey/black

$59 \mathrm{mg}$ grey and black specks

$45 \mathrm{mg}$ grey and black specks

$9 \mathrm{mg}$ slight greyish

$30 \mathrm{mg}$ light grey to black

$11 \mathrm{mg}$ very dark, black

$8 \mathrm{mg}$ very dark, black

Dark grey and black residues tend to occur in samples which later displayed yellow-orange solution colors and are inferred to represent unoxidizable Carbon... Greyish-white residues are more likely to be siliceous matter.

Note that residue masses are somewhat irrepresentative of original matter because of air-drying, whereas original samples were sometimes quite wet. The mass of the residues was determined by difference using an average of masses determined for clean $0.22 \mu m$ millipore membranes, hence these are approximate but reasonably reliable. 
pH Titration of Sample ID 111293-22

AN, 1-8-94, 1 min/reading, $10 \mathrm{~g}$ sample/5O mL H2O

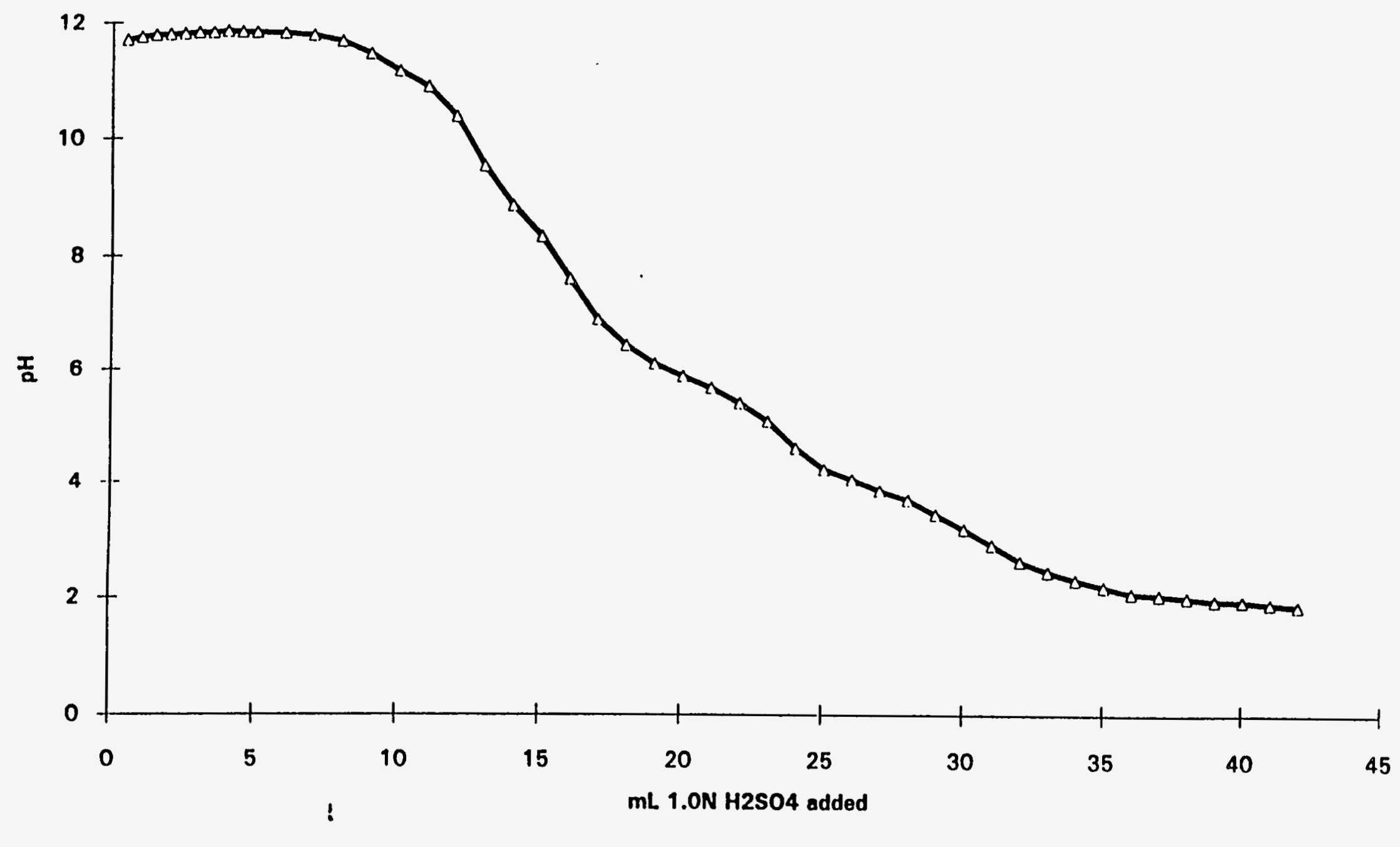

DH Titration Curses 


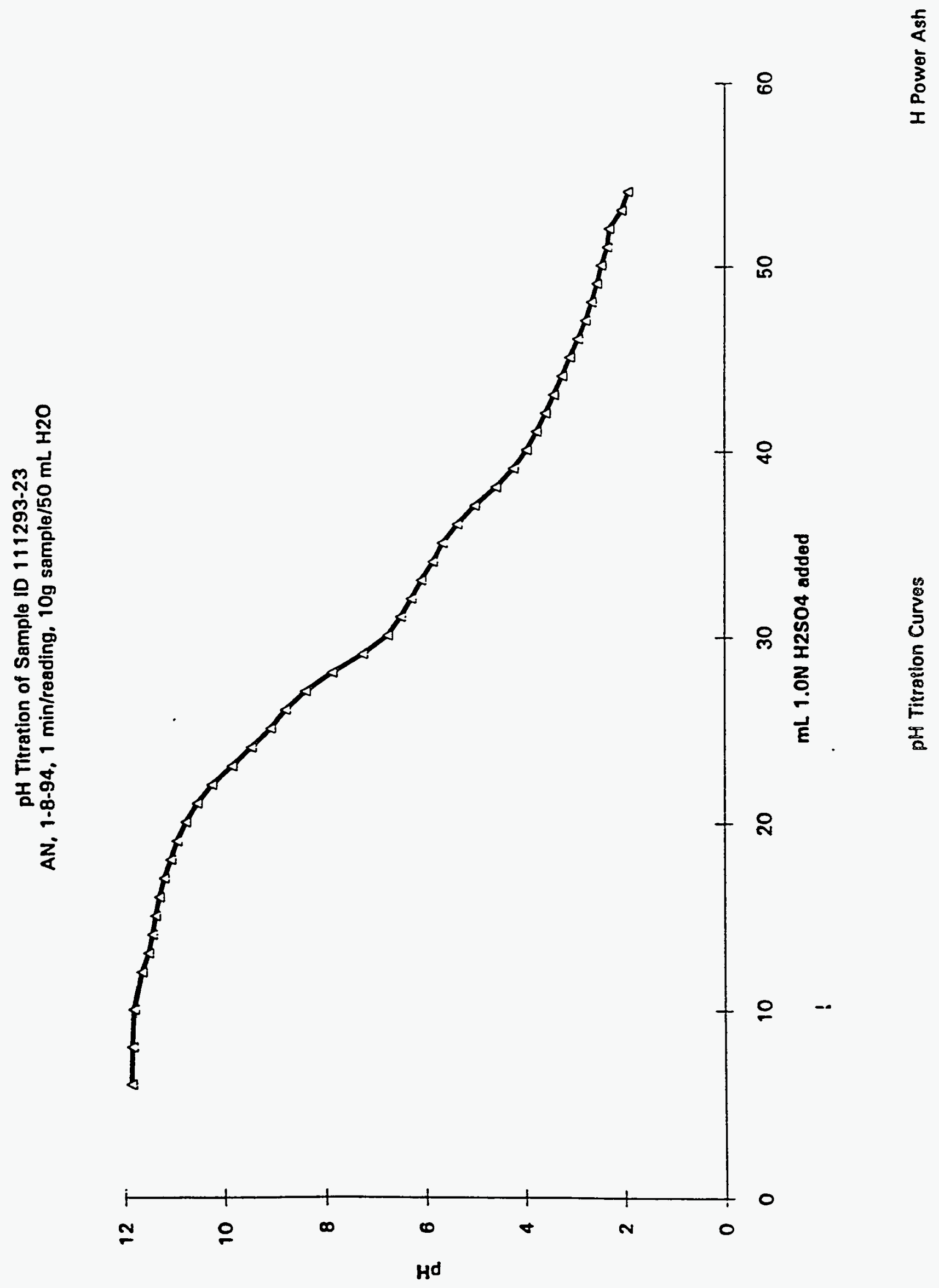




\section{pH Titration of Sample 10 111293-24}

AN, 1-8-94, $1 \mathrm{~min} / \mathrm{reading}, 10 \mathrm{~g}$ sample/5O mL H2O
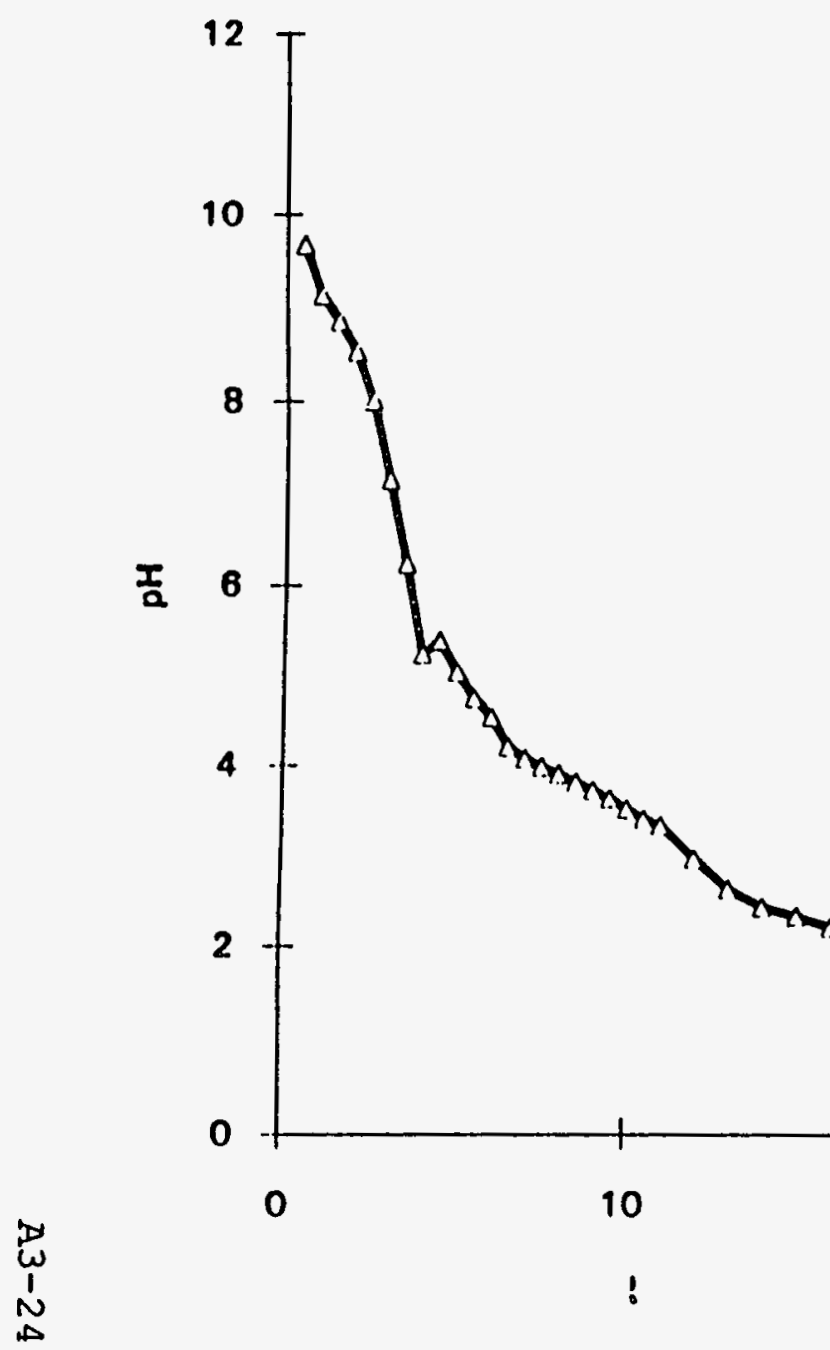

!

20 mL. 1.ON H2SO4 added 
pH Titration of Sample ID 111293.24

AN, 1-8-94, 1 min/reading, 10 g sample/50 mL H2O

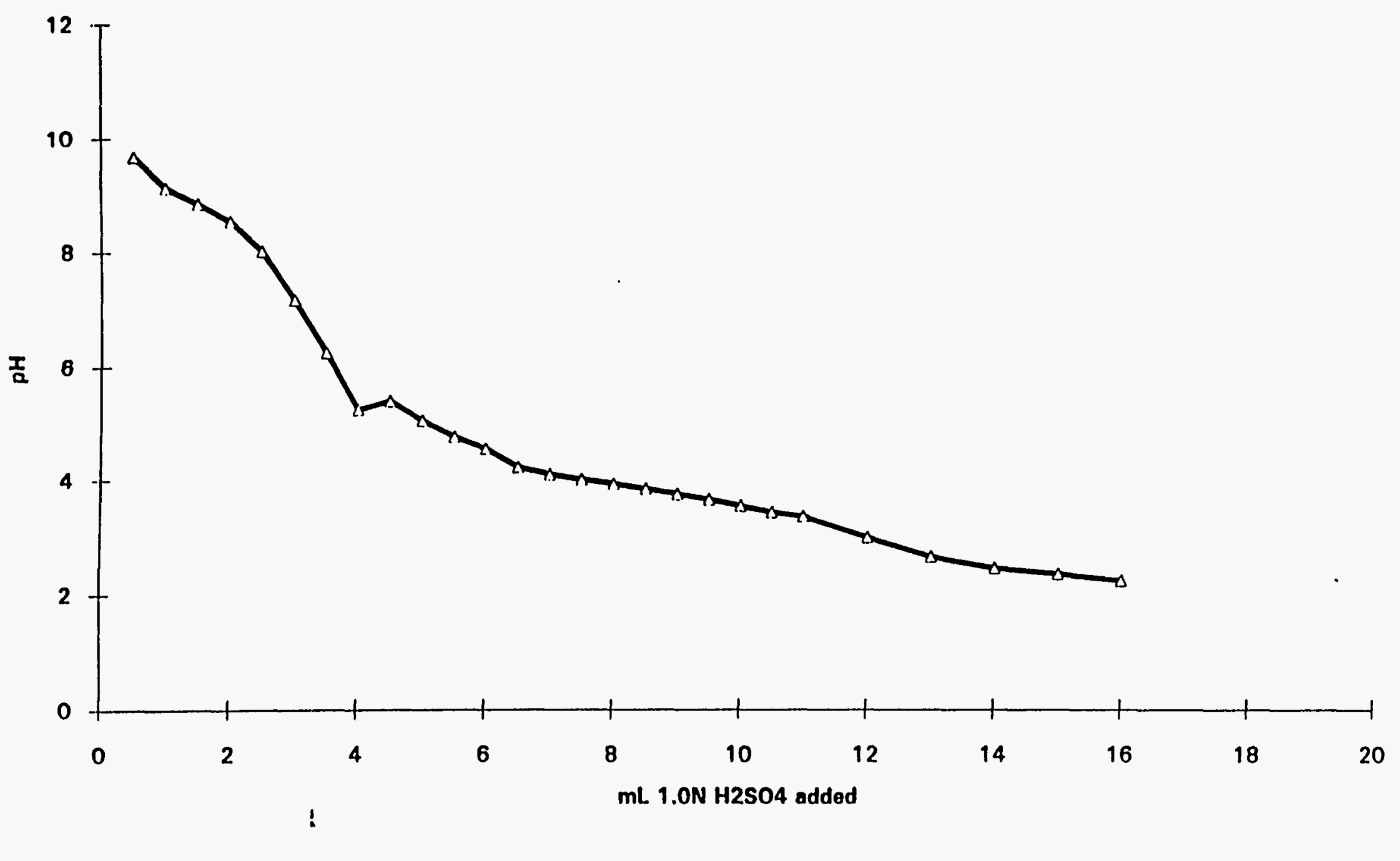

pH Titration Curves 
离

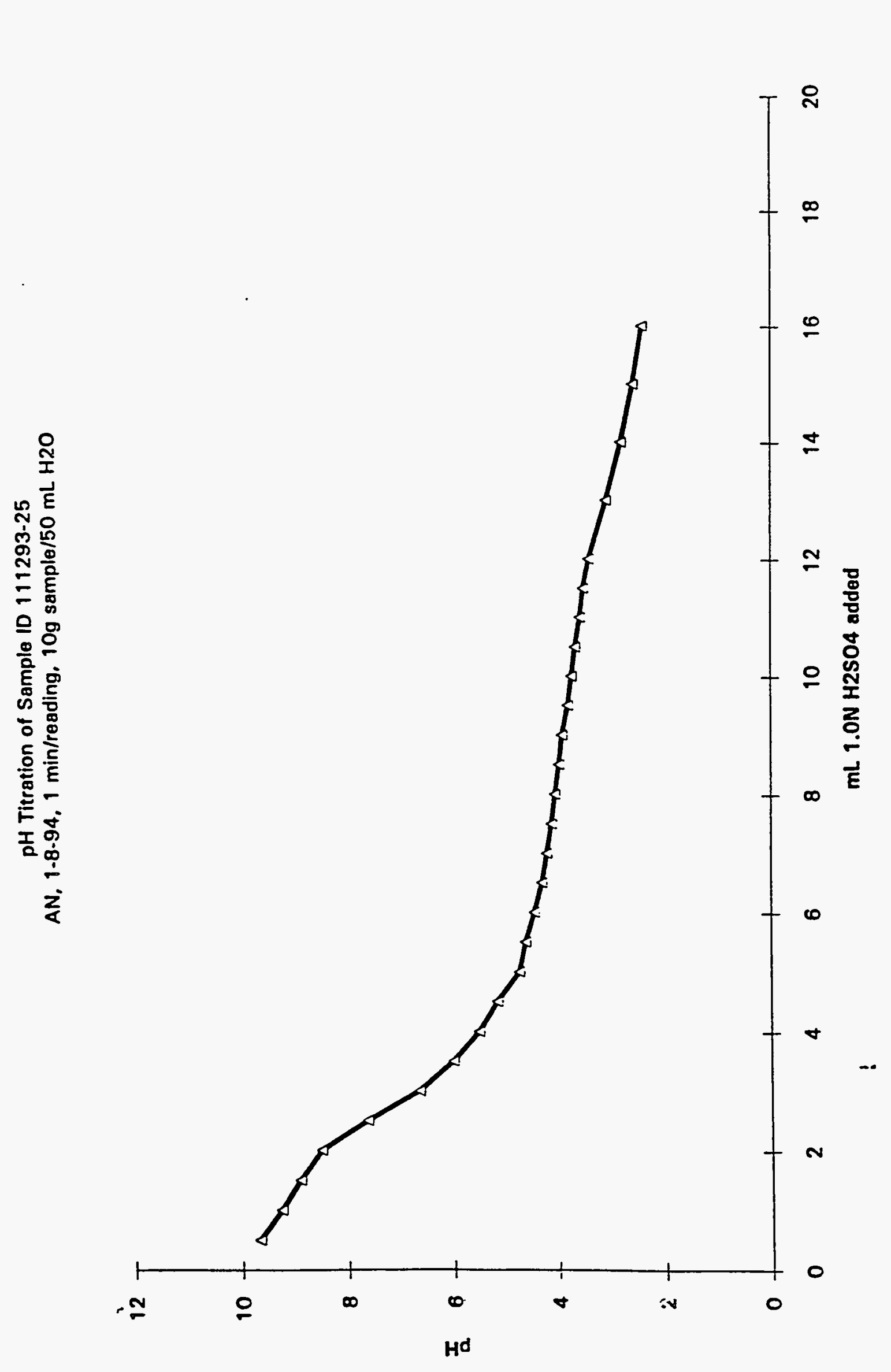

ن.
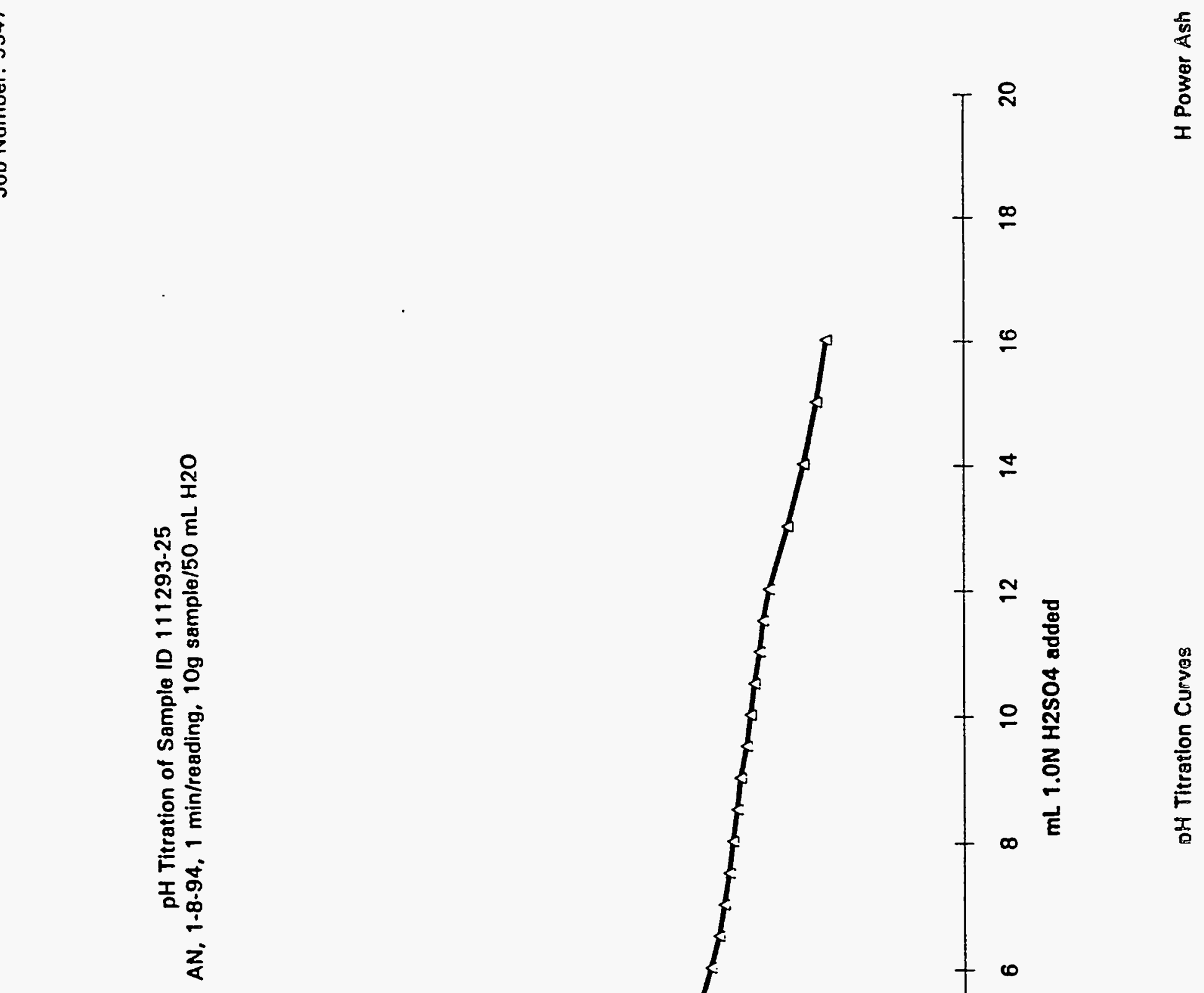


\section{pH Titration of Sampla ID 111293-26}

AN, 1-8-94, 1 min/reading, $10 \mathrm{~g}$ sample $/ 50 \mathrm{~mL}$ H2O

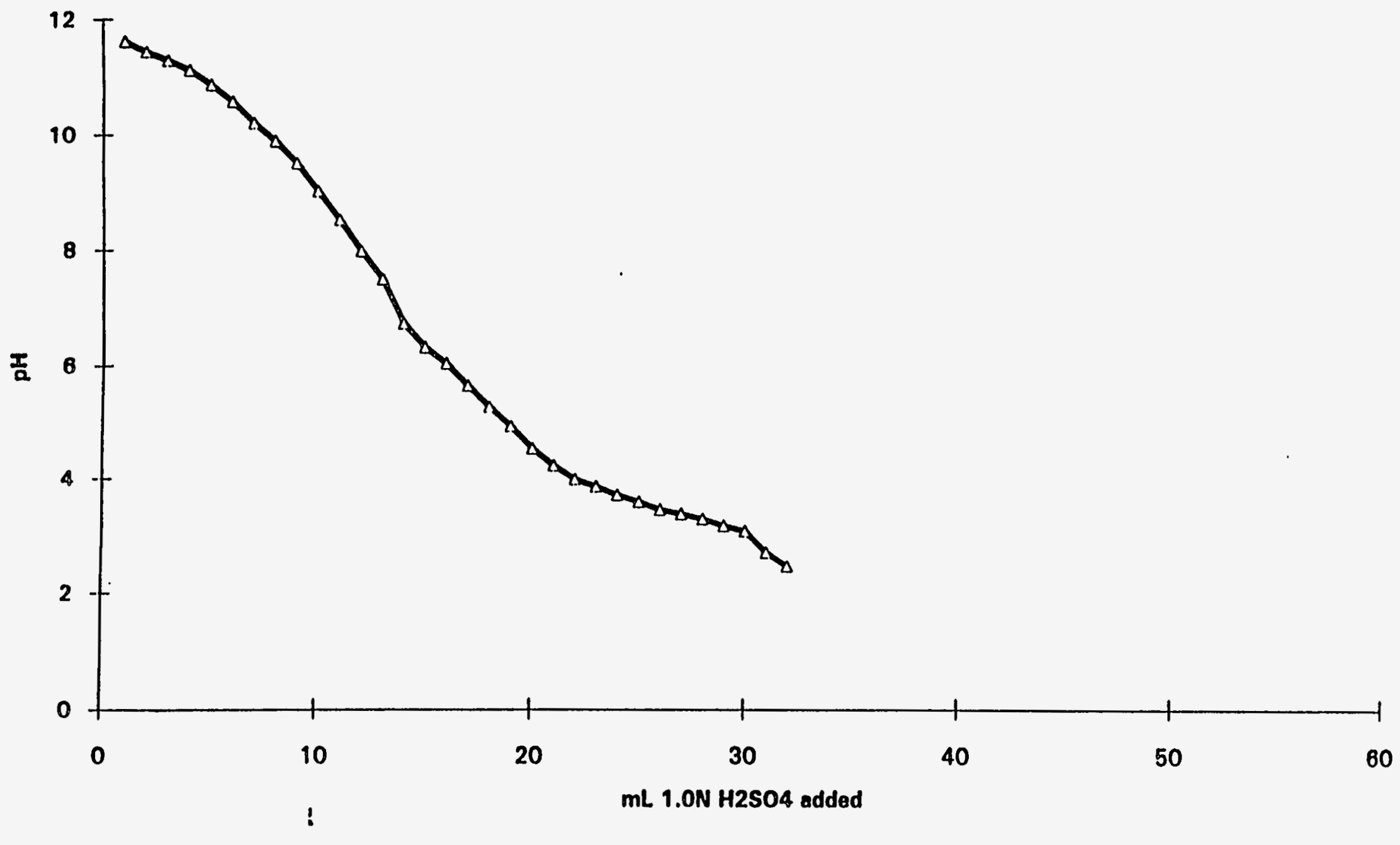




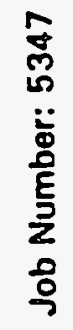

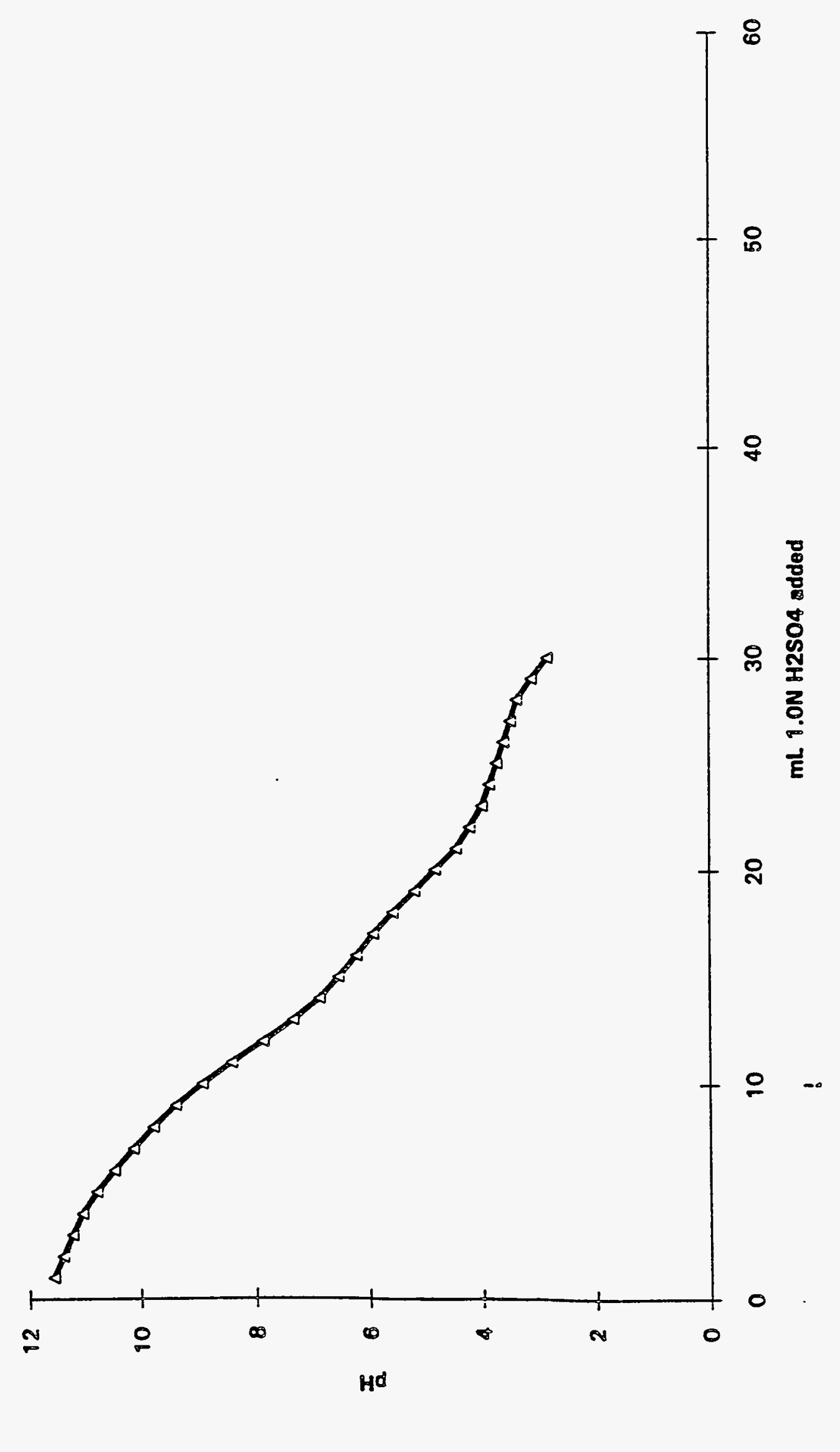

A3-28 
E.L. Pacific

pH Titration of Sample ID 111293-28

AN, 1-12.94, 1 min/reading, $10 \mathrm{~g}$ sample $/ 50 \mathrm{~mL} \mathrm{H} 2 \mathrm{O}$

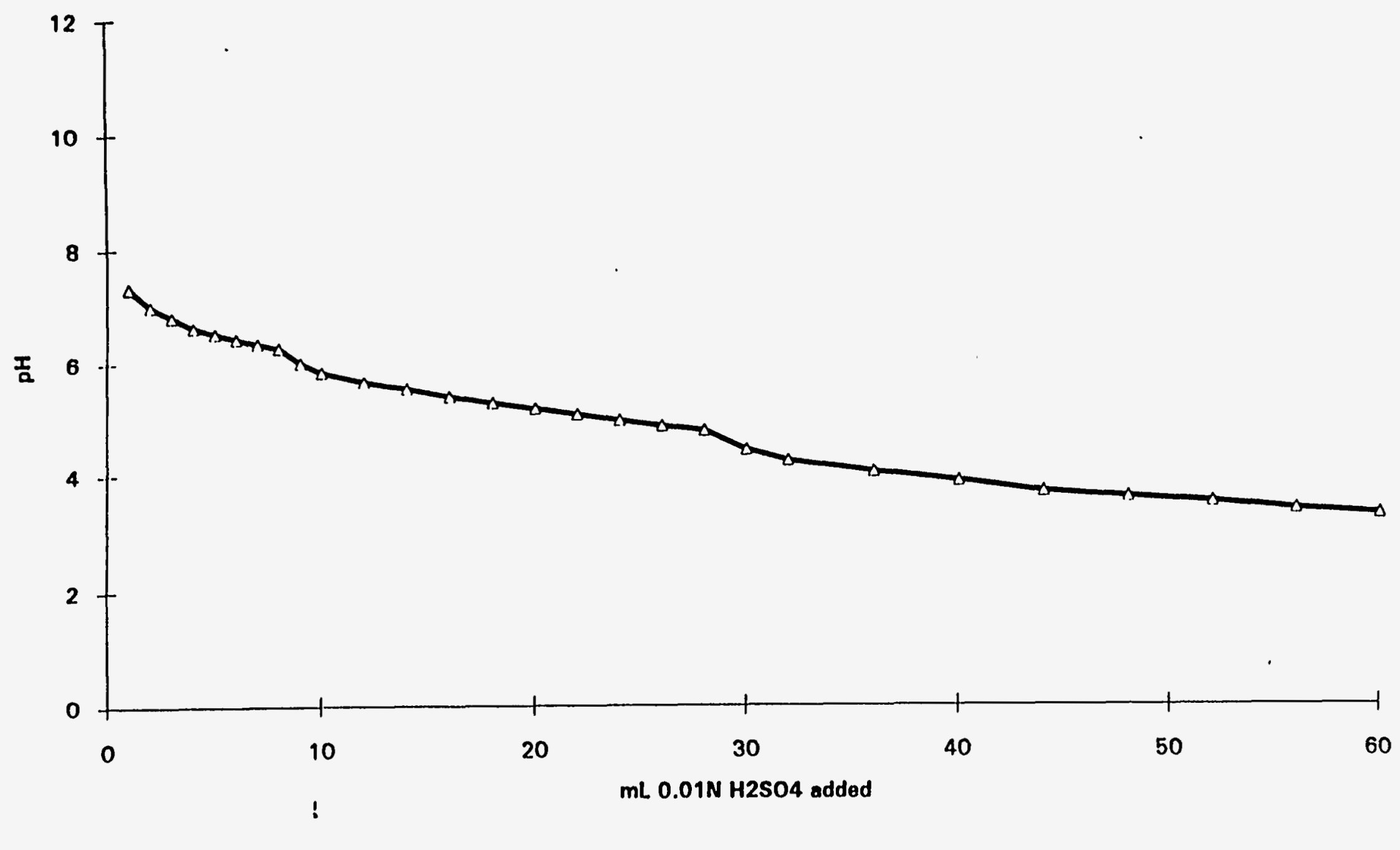

pH Titration Curves 
pH Titration of Sample ID 111293-29

AN, 1-11-94, $1 \mathrm{~min} /$ reading, $10 \mathrm{~g}$ sample/50 $\mathrm{mL} \mathrm{H} 2 \mathrm{O}$

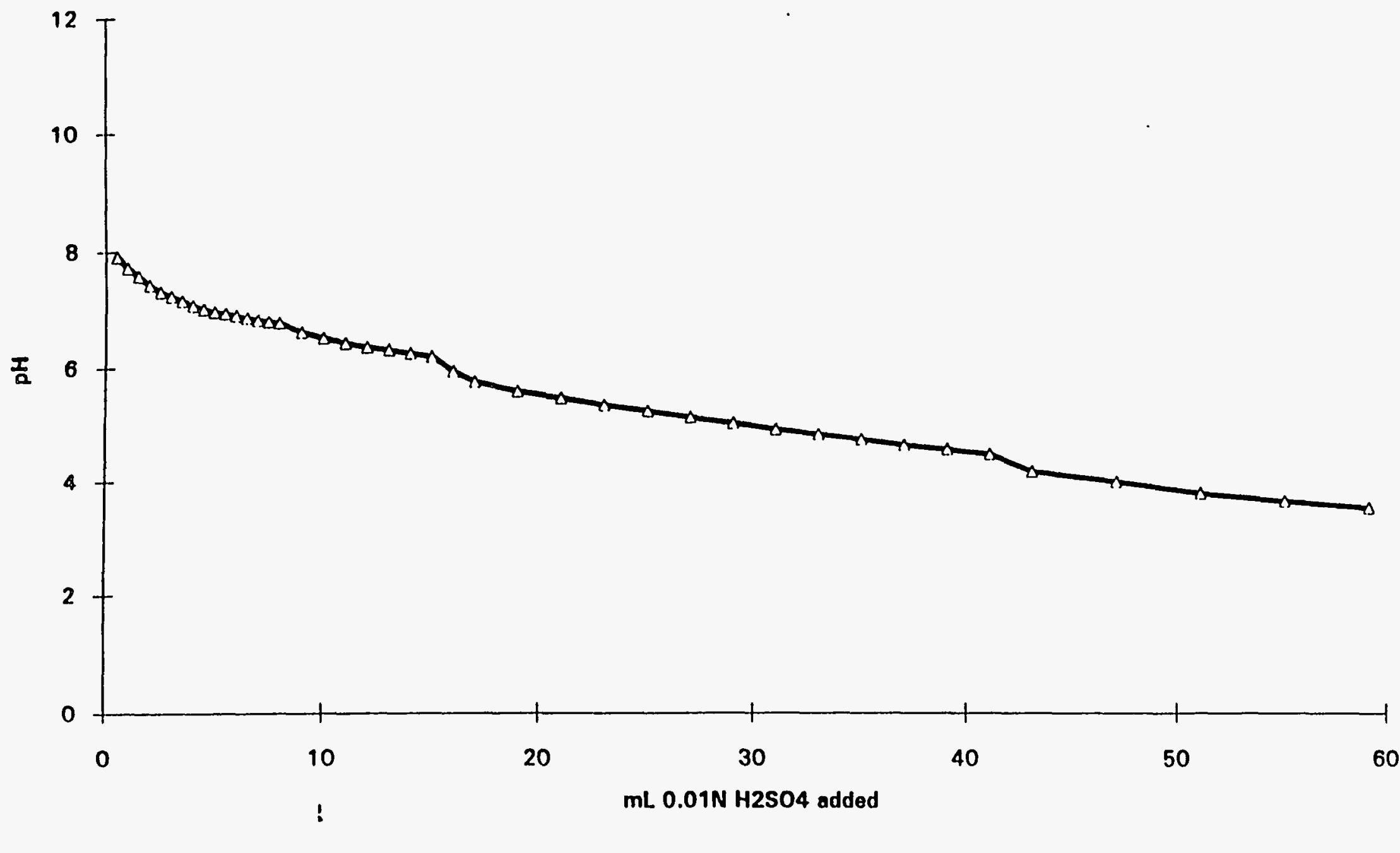




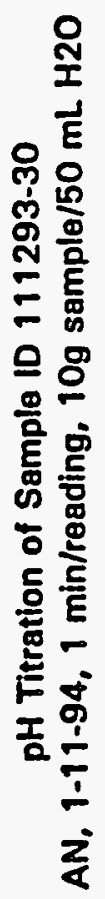

ن.

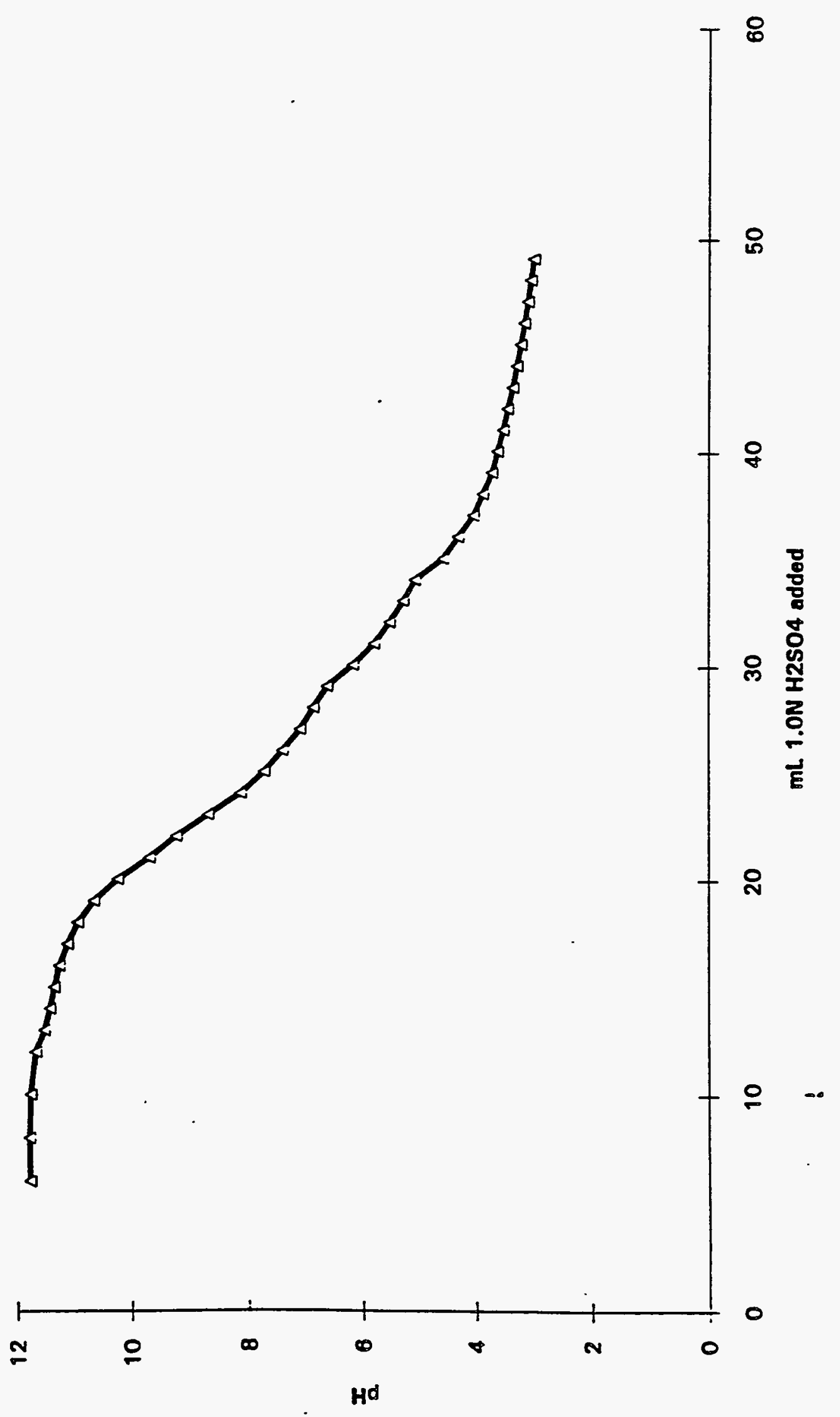




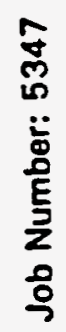

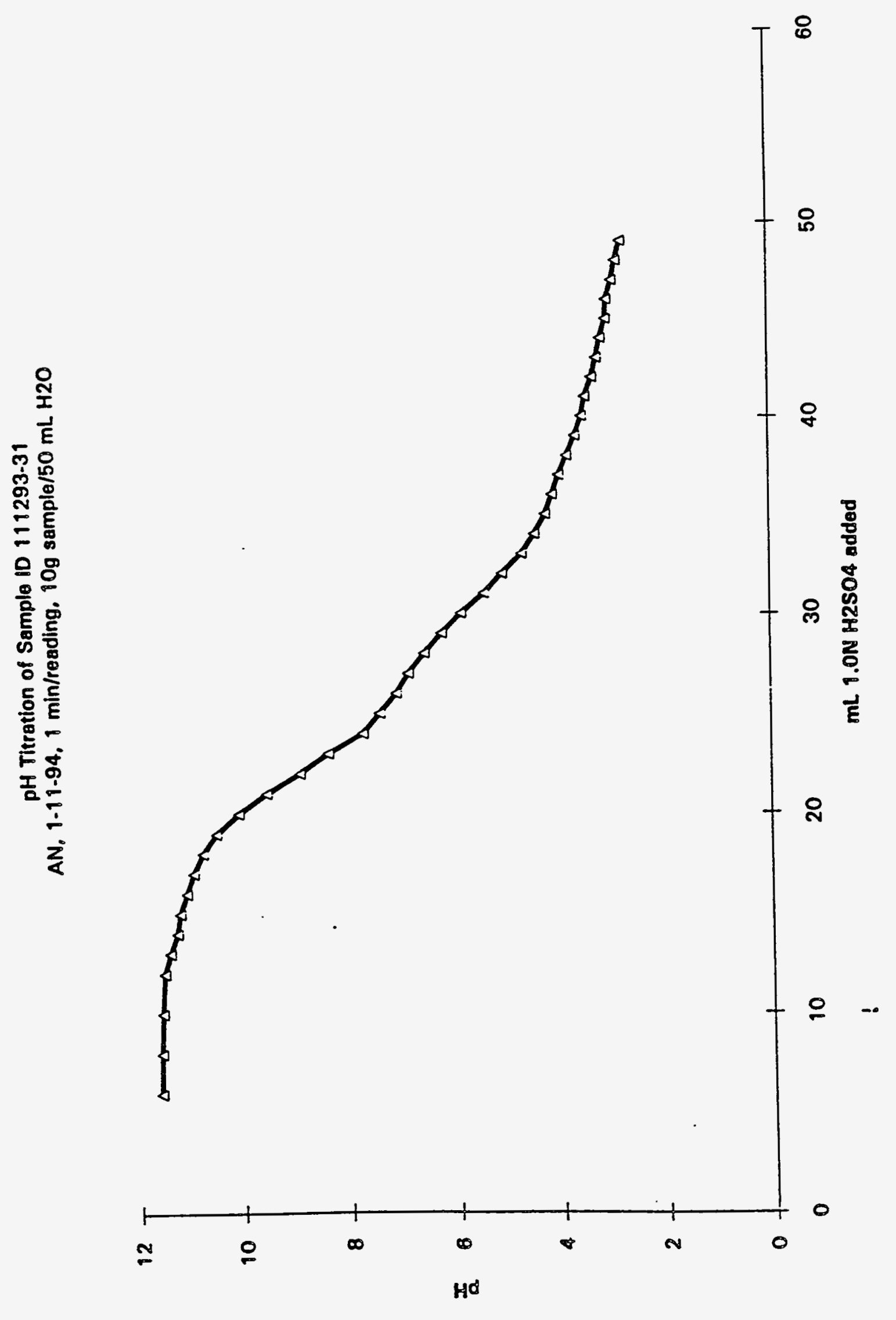


pH Titration of Sample ID 111293-32

AN, 12-29, $1 \mathrm{~min} /$ reading, $10 \mathrm{~g}$ sample $/ 50 \mathrm{~mL} \mathrm{H} 2 \mathrm{O}$

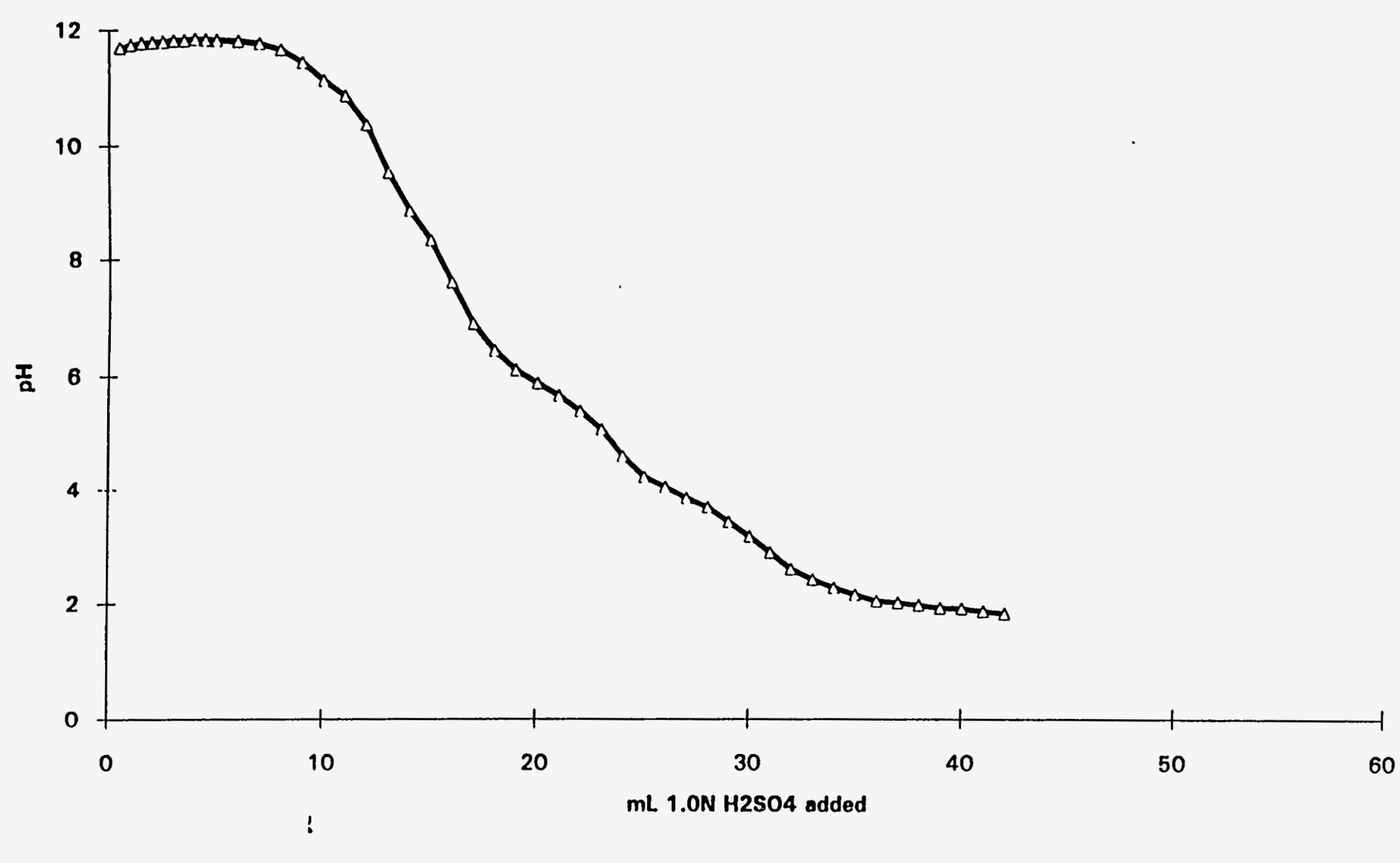

pH Titration Curves 
pH Titration of Sample ID 111293-33

AN, 12-29-93, $1 \mathrm{~min} /$ reading, $10 \mathrm{~g}$ sample $/ 50 \mathrm{~mL}$ H2O

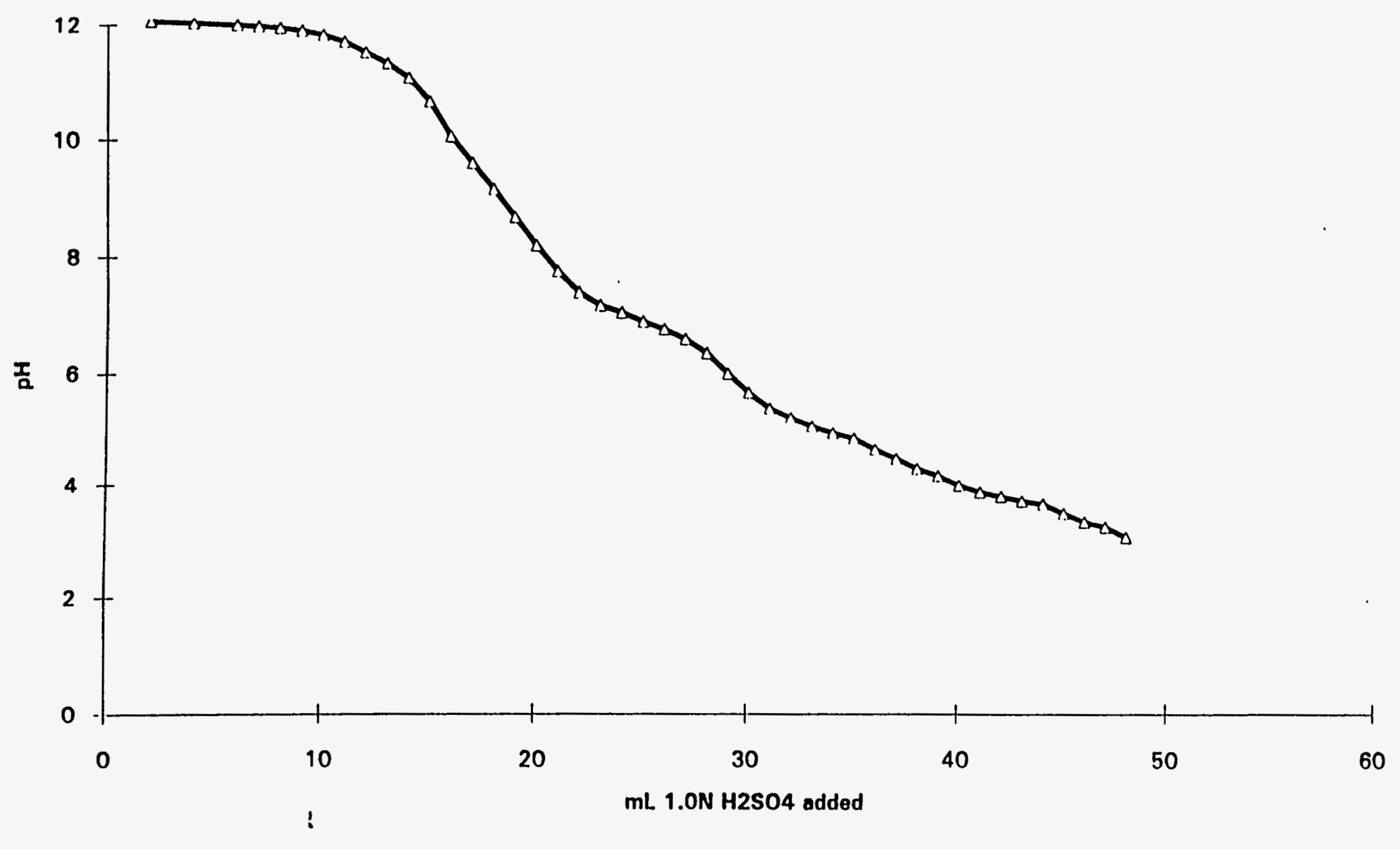




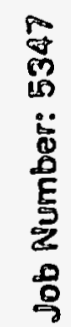

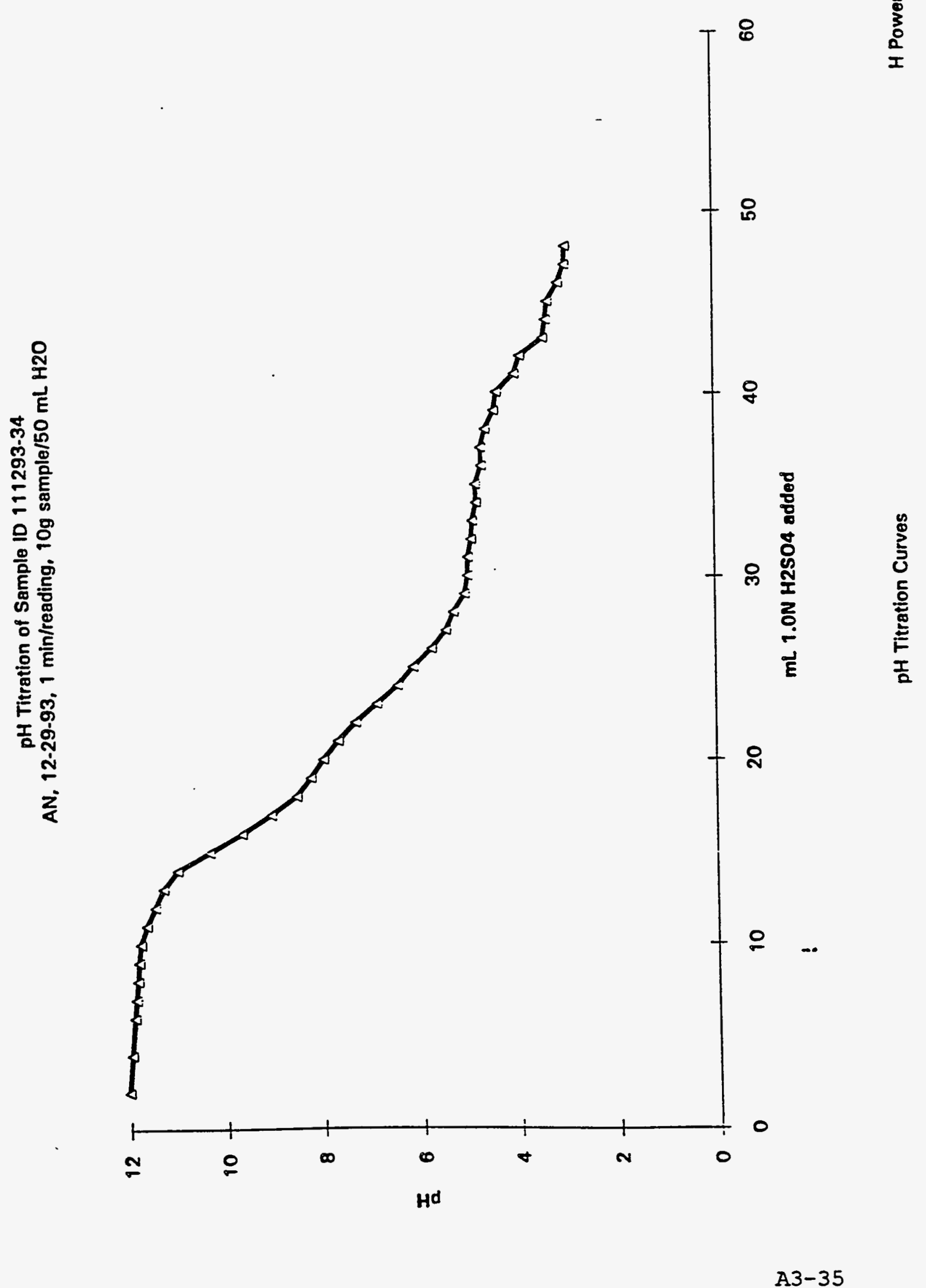

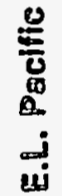




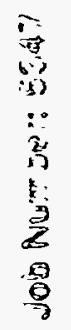

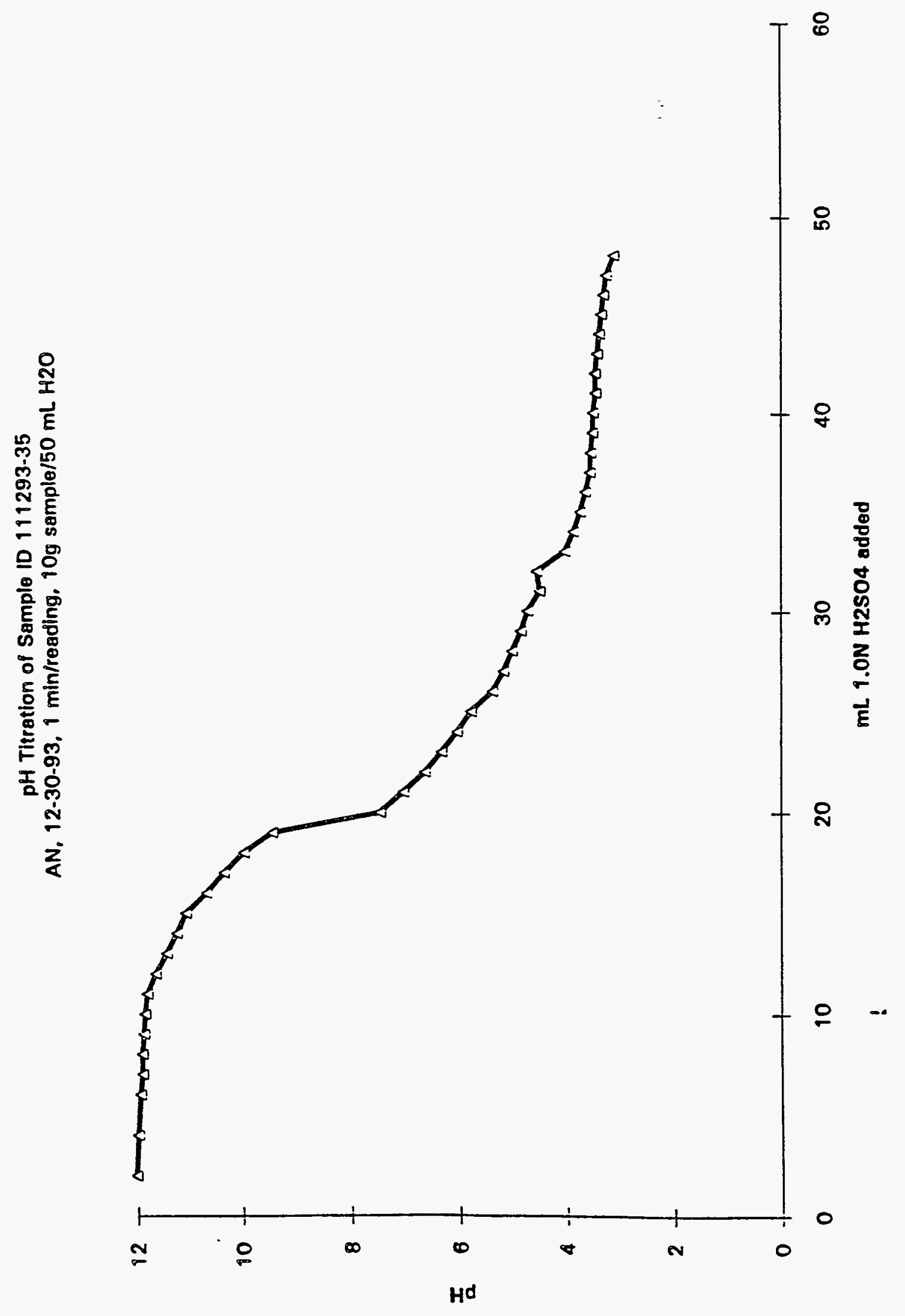

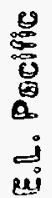

A3-36 


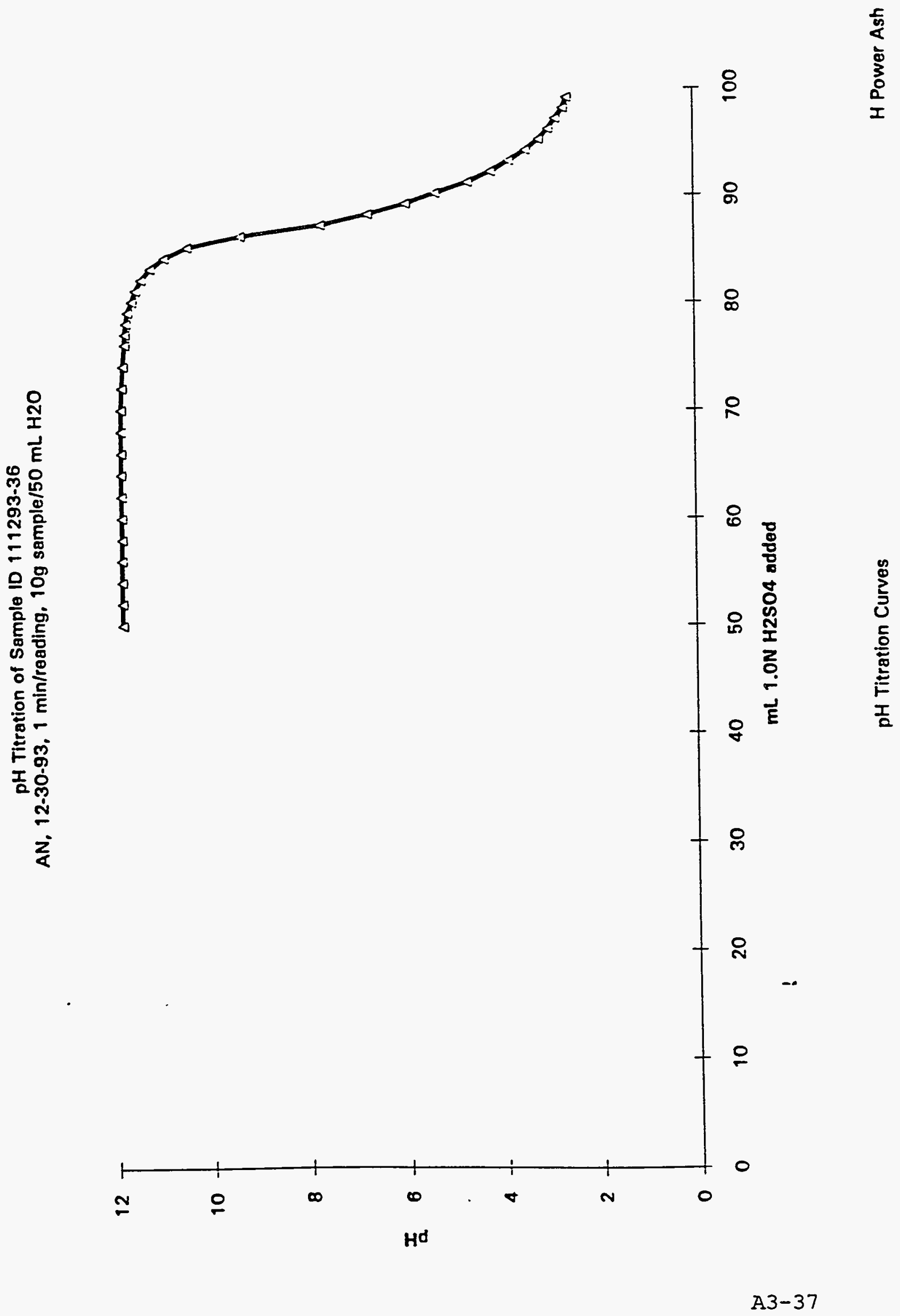




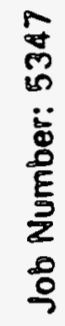

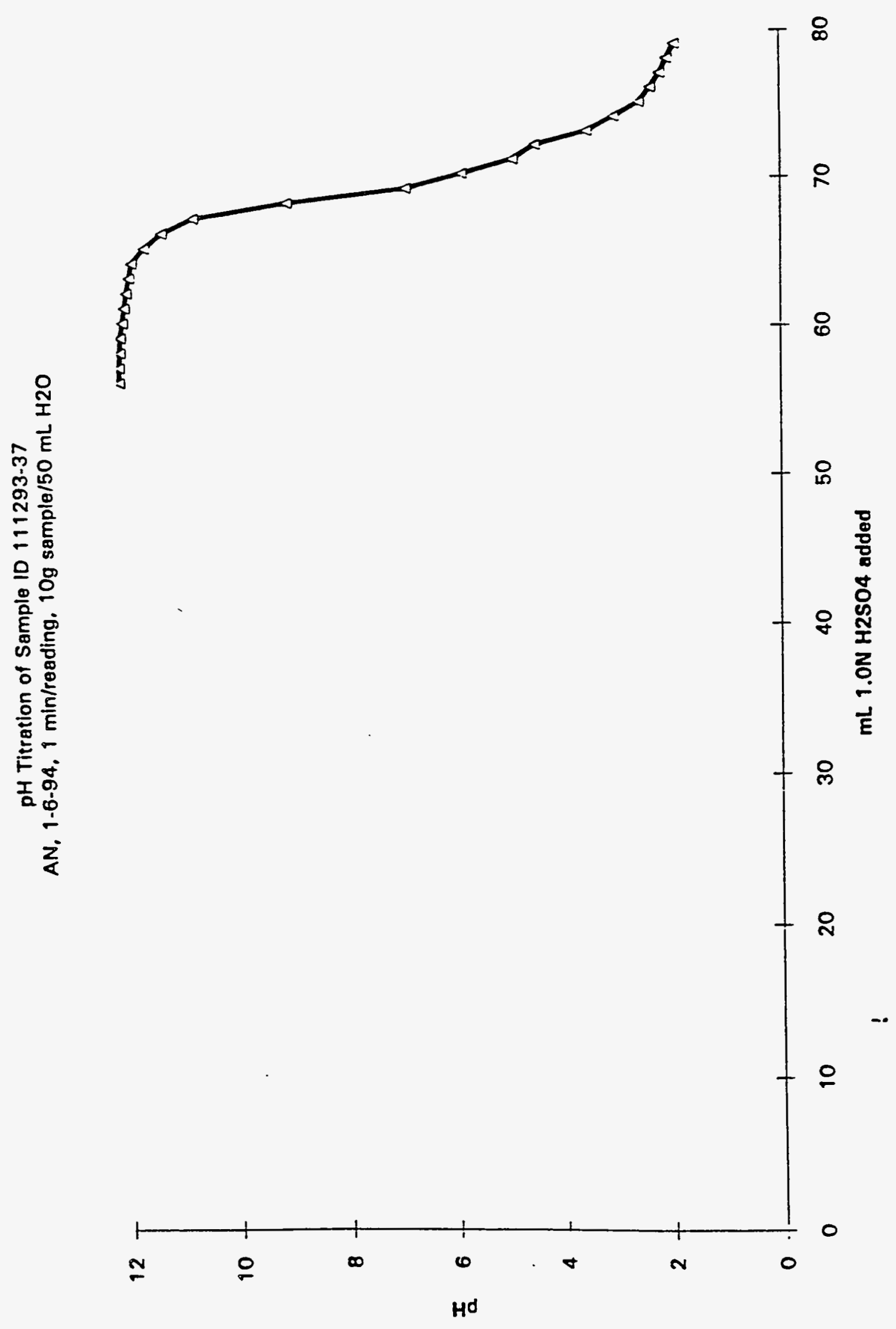

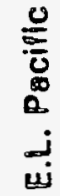


pH Titration of Sample ID 111293-38

AN, 1-6-94, 1 min/reading, $10 \mathrm{~g}$ sample/50 $\mathrm{mL} \mathrm{H} 2 \mathrm{O}$

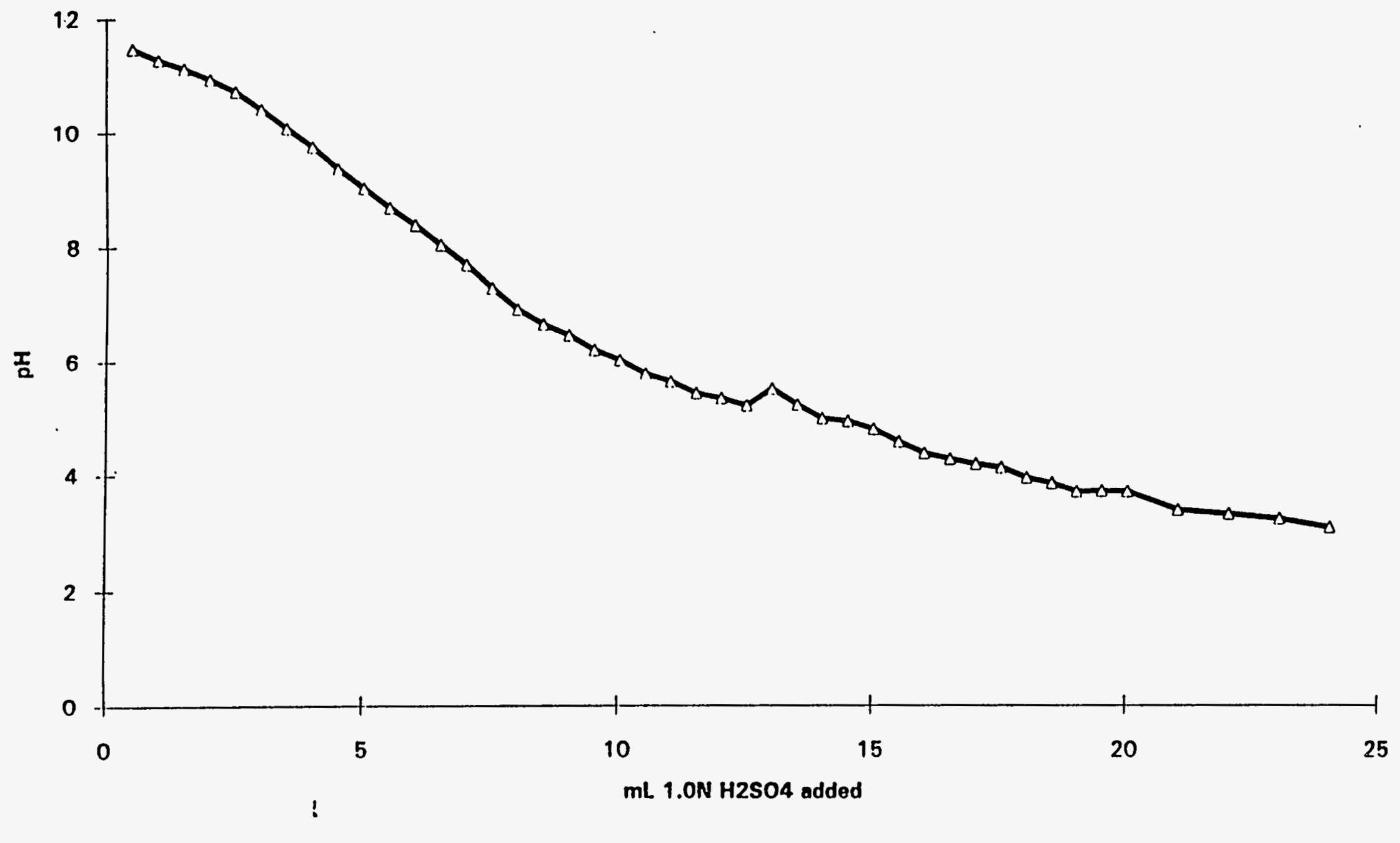


Enumeration of Fecal Coliform Densities in H-Power Ash and Municipal Sludge Mixtures Water Quality Laboratory

November 19, 1993

\section{Sample Description}

Sand Island sludge

H-Power Bottom Ash

Replicate 2.5

Replicate 2.6

Soil

Replicate 4.5

Replicate 4.6

H-Power Ply Ash and Sludge (3:1 Mix)

Replicate $\mathbf{5 . 5}$

Replicate 5.6

B-Power Bottom Ash and Sludge (3:1 Mix)

Replicate 6.5

Replicate 6.6

B-Power Fly Ash and Sludge (2:2 kix)

Replicate 7.5

Replicate 7.6

B-Power Fly Ash and Sludge and Lime (2:2:1 Kix)

Replicate 8.5

Replicate 8.6

Waipahu Combined Ash

Replicate 9.5

Replicate 9.6

\section{MPN/gram dry weight}

$$
5,050
$$

$<0.24$

$<0.24$

0.66

0.39

$<0.38$

$<0.38$

$<0.40$

$<0.40$

$<0.49$

$<0.49$

$<0.42$

$<0.42$

$<0.26$

$<0.27$ 
Enumeration of Fecal Coliform Densities in H-Power Ash ...

Page 2 of 3 .

\section{MATERIALS AND METHODS}

Sample source and handling. All samples were delivered to the Water Quality Laboratory by personnel or consultants from the H-Power facility. The one (1) sludge sample received on 11-8-93 at 12:30 PM was not accompanied by a chain-of-custody form and an appropriate transport temperature of $0-10^{\circ} \mathrm{C}$ was not maintained. The fourteen (14) samples received on 11-12-93 at 1:28 pM were accompanied by a chain-of-custody form, but a spot check revealed that appropriate transport temperatures were not achieved for all sample containers. An unexpected sample (Mixture 9 - Waipahu ash) was received while an expected sample (Mixture 1 - H-Power fly ash) was not received. The samples received and analyzed for fecal coliform include the sludge and two replicates each of Mixtures $2,4,5,6,7,8$, and 9 .

Upon receipt, samples were immediately placed into a refrigerator at $4^{\circ} \mathrm{C}$ and sequentially removed for processing. All samples were analyzed within the recommended six (6) hours after collection except for samples 8.5 and 8.6 (about 6.5 hours) and samples 9.5 and 9.6 (about 9 hours after collection at $\mathrm{H}$-Power).

Bacteriological analyses. The solid and semi-solid mixtures were analyzed for fecal coliform using the Most Probable Number (MPN) method according to procedures detailed in "Control of pathogens and Vector Attraction in Sewage Sludge," EPA/625/R-92/013, December 1992, Appendix F. Briefly, a sample dilution series was prepared and inoculated into tubes of Lauryl Tryptose Broth

(ITB). The 10.0 and 1.0 gram dilutions were weighed and directly added. The 0.1 gram dilution was prepared by adding 10.0 grams of sample to $90.0 \mathrm{~mL}$ of buffered dilution water and blending for five minutes ( $1.0 \mathrm{~mL}$ of this solution contains 0.1 gram sample). Ten-fold serial dilutions starting with this solution were also prepared as required. Positive Escherichia coli and negative Enterobacter aerogenes control cultures were inoculated into LTB tubes. AIl ITB tubes including uninoculated blank control tubes were incubated at $35.0^{\circ} \mathrm{C}$ for 24-48 hours. Growth in tubes with positive gas production was transferred to EC broth and incubated for 24 hours at $44.5^{\circ} \mathrm{C}$. Positive gas production confirmed the presence of fecal coliform. Sterile supplies and media and aseptic techniques were employed. Replicate analyses of samples 2.5 and 2.6 were also performed.

pH correction. Sample mixtures with ash or lime exhibited extremely high pH while the sludge sample exhibited slightly low $\mathrm{pH}$. Such $\mathrm{pH}$ extremes could interfere with the growth of fecal coliform under test conditions and cause erroneous results. Additional 1.0 gram dilutions into LTB were prepared for all samples and the $\mathrm{pH}$ determined - which ranged from $\mathrm{pH} 6.89$ to pH 7.84. An additional 10.0 gram dilution of sample 8.6 
Enumeration of Fecal roliform Densities in H-Power Ash ...

Page 3 of 3 .

(ash/sludge/lime mixture) into double-strength LTB was prepared, washing with $10.0 \mathrm{~mL}$ sterile distilled water, and the $\mathrm{pH}$ was determined at 11.05. The $\mathrm{pH}$ buffering capacity of the LTB solutions sufficiently corrected the high and low $\mathrm{pH}$ of the samples at the 1.0 gram dilution but not at the 10.0 gram dilution. Results from 10.0 gram dilutions were not used (except for soil samples 4.5 and 4.6 at $\mathrm{pH} 8$ before dilution).

Percent solids. The final calculation in terms of grams of dry weight required the determination of percent solids. A 10-50 gram aliquot was dried at $104^{\circ} \mathrm{C}$ and weighed, then re-dried until a constant weight of less than or equal to $50 \mathrm{mg}$ between each subsequent weighing was achieved. Empty dishes were weighed at the beginning and end of each series as weight control checks. Duplicate analyses for percent solids was performed for each sample and the average result used for final calculations.

Calculations. The following equation is used to calculate fecal coliform densities:

$$
\text { MPN fecal coliform/gram }=\frac{10 \times \text { MPN index } / 100 \mathrm{~mL}}{\text { largest volume } \times \div \text { dry solids }}
$$

Sample disposal. The dried samples from the percent solids determinations and any remaining sample mixtures were consolidated and transferred to the Treatment and Disposal Division for final disposal into the municipal landfill. 


\section{Food Quality Labs}

1505 Dillingham Blvd., Suite 220

Honolulu, Hawaii 96817

Tel/Fax: 808-841-4484

For: H-Power Ash Utilization Study

91-714 Hanua St.

Kapolei, HI 96707

Case No. 1

Received: $11-12-93$

Analyzed: 11-12-93

Completed:11-16-93

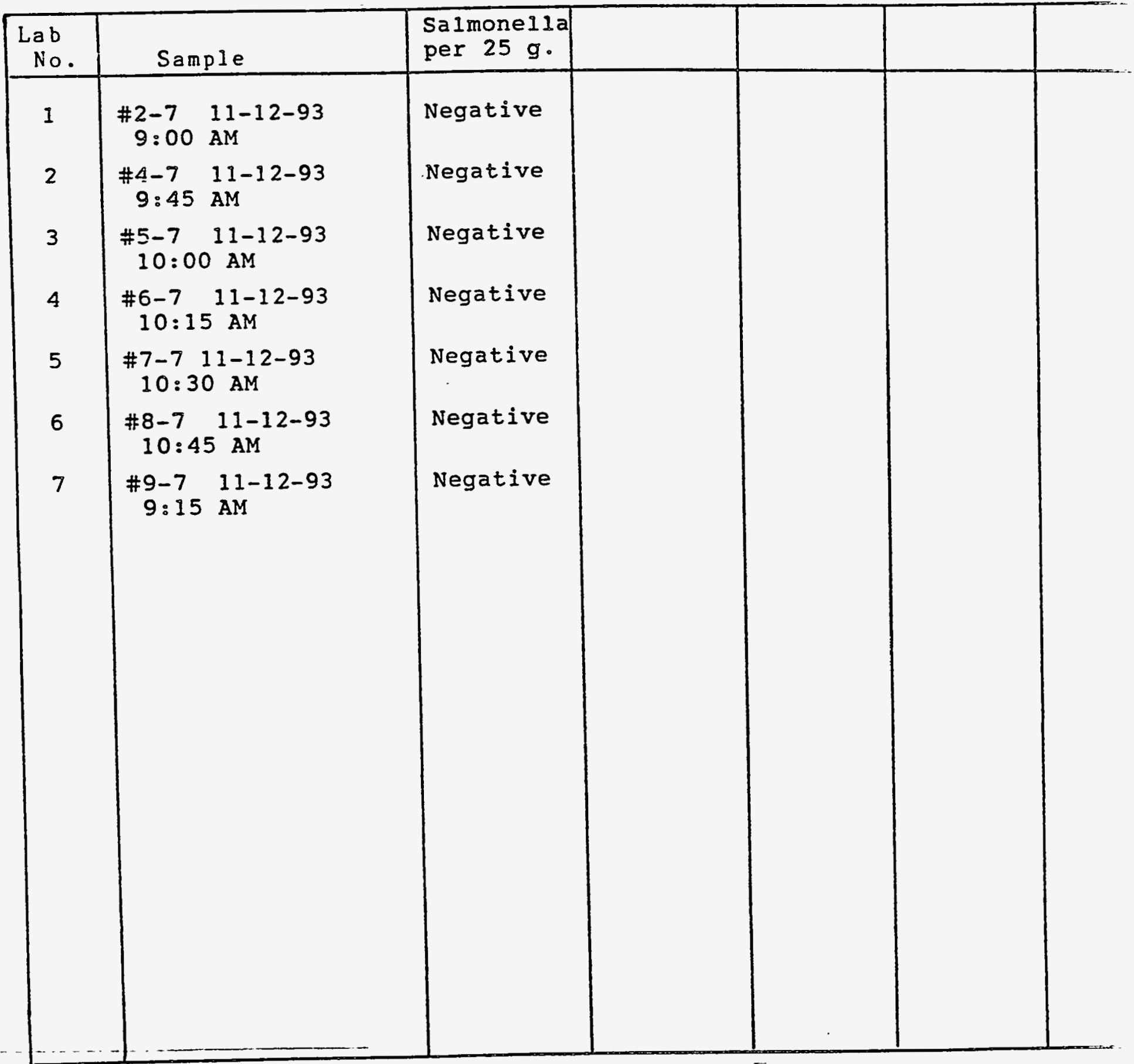

Analyzed by:

Unu Javer 


\section{Appendix A5}

\section{Botanical Growth Potential of Ash Mixtures}

This subtask was designed to determine the potential of the ash mixtures to support botanical growth. This was accomplished by seeding mixtures with native grasses and recording germination rates, disease, color, etc. In addition to seed, similar experiments were also carried out using transplanted turf grass. It was originally planned that the mixtures be used in three varying degrees: one at full concentration, and two at lower concentrations as soil admixtures. However, due to time constraints and insufficient resources, tests on the latter two concentrations were not conducted.

\section{Procedure}

The following six turfgrass samples were selected:

\section{Sunturf Bermuda Seashore Paspalum \\ 'Z-3' Zoysia}

Tifway 419 Bermuda

'El Toro' Zoysia

'Emerald' Zoysia

Each sample, approximately $58 \mathrm{~cm}^{2}\left(9 \mathrm{in}^{2}\right)$ in size, was placed in individual 10-centimeter (4-inch) pots; each containing one of the selected MWC ash mixtures. Four replicates of each sample were prepared.

The following three types of turfgrass seeds were selected:

Zoysia Japonica

Hulled Bermuda Grass

Australian Carpet Grass

Each seed type was sown in a 10-centimeter (4-inch) pot containing one of the selected MWC ash mixtures. Four replicates of each sample were also prepared. All of the samples were provided with water. No fertilizers, pesticides, etc., were used or added. Observations were recorded daily and photographs taken as needed

The following report, prepared by the staff of the City \& County of Honolulu's Botanical Gardens, includes the actual observation data, and the conclusions drawn therefrom by the horticulturists. Photographs of the test specimens begin on page A5-6, following the report. 


\section{INITIAL H-POFER ASH UTILIZATION STUDY}

HONOLULU BOTANICAL GARDENS

MATERIALS AND METHODS USED

12-28-93 Planted 6 turfgrass samples:

Sunturf Bermuda

Tifway 419 Bermuda

Seashore Paspalum

'El Toro' Zoysia

' $\mathrm{z}-3$ ' Zoysia

'Emerald' Zoysia

Four samples of each turfgrass (approximately 3" square inches each) were placed in 4" pots with soil samples \# $4,5,6,7,8$ provided by H-Power.

Plants were only provided with water - no fertilizers, pesticides, etc. were used.

1-6-94 Three types of turfgrass seed were sown:

Zoysia japonica

Hulled Bermuda Grass

Australian Carpet Grass

Four pots (4") of each seed type were sown over soil samples $\# 4,5,6,7,8$ provided by $H$-Power.

Pots were only provided with water - no fertilizers, pesticides, etc. were used.

\section{OBSERVATIONS}

$1-6-94$ - NO CHANGES IN TURF POTS - PLANTS ALL GREEN
$1-13-94-$

1-13-94 - SEED SPROUTING : \#4 HULLED BERMUDA GRASS

\# ZOYSIA JAPONICA

(4 POTS)

(2 POTS)

$1-14-94="$ " $"$ " \#8 HULLED BERMUDA GRASS

\#4 ZOYSIA JAPONICA

(3 POTS)

(4 POTS)

$1-18-94=" 1$ " $\quad$ " \#4 ZOYSIA JAPONICA

\#4 AUSTRALIAN CARPET

(\$ POTS)

\#8 HULLED BERMUDA

\#6 HULLED BERMUDA

(4 POTS)

(3 POTS)

(1 POT) 


$\begin{array}{llllll}1-18-94- & \text { SEED SPROUTING : } & \# 6 \text { AUSTRALIAN CARPET } & \text { (1 POT) } \\ 1-19-94- & \text { " } & \text { " } & \text { " } & \text { " } & \text { NO CHANGE } \\ 1-20-94- & \text { " } & \text { " } & \text { " } & \text { NO CHANGE } \\ 1-21-94- & - & \text { " NO CHANGE }\end{array}$

NO SIGNIFICANT CHANGES NOTED UNTIL 2/15

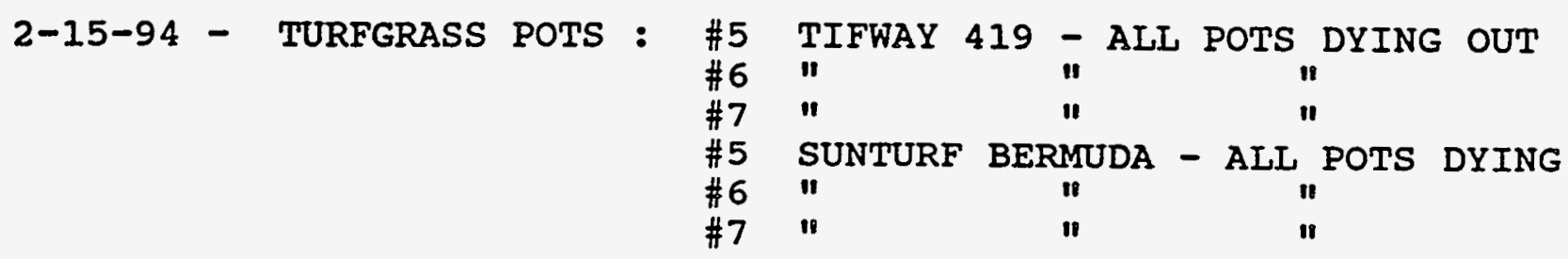

2-22-94 - TURFGRASS POTS : ALL SOILS - SEASHORE PASPALUM

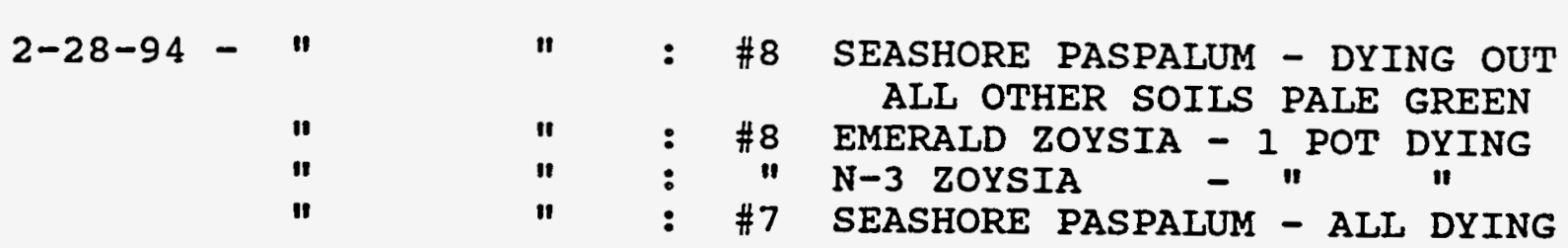

CONCLUSIONS

FOR COMPLETE RECORD PLEASE SEE ATTACHED DATA SHEET WITH DATES. GERMINATION FOR THE SEEDS WAS POOR IN ALL CASES EXCEPT FOR THE AUSTRALIAN CARPET GRASS WHICH GERMINATED BETTER THAN THE CONTROL SOIL. EVENTUALLY AIL SAMPLES DIED DUE EITHER TO FACTORS ASSOCIATED WITH THE MEDIA CHEMICALLY (ALKALINITY?) OR STRUCTURALLY (VERY POOR DRAINAGE) - ALL SEED POTS DEAD AS OF $3 / 94$.

THE TURFGRASS POTS HAVE ALL DIED AS OF 3/13/94. THE PLANTS WOULD BEGIN TO TURN PALE GREEN AND EVENTUALLY BURN OUT TO A YELIOW COLOR. DRAINAGE WAS SO POOR THAT ROOT GROWTH WAS NOT SUBSTANTIAL. THE PLANTS MOST LIKELY IIVED ON THE ORIGINAL TOP SOIL THAT THEY WERE PIANTED WITH AND COULD NOT SUSTAIN LONG TERM GROWTH IN THE HARDENED, CEMENT-LIKE SOIL SAMPLES DUE TO LACK OF WATER DRAINAGE, OXYGEN, AND POSSIBLY THE ALKALINE PH.

OF ALL TYPES TESTED THE AUSTRALIAN CARPET GRASS PERFORMED THE BEST, EVEN GERMINATING IN SEED TRIAL BETTER THAN THE CONTROL. HOWEVER, NONE OF THE SOILS WERE SUITABLE FOR IONG TERM GROWTH. WE CANNOT DETERMINE WHAT FACTORS ARE RESPONSIBLE, BUT THE SOIL STRUCTURE ALONE IS CAUSE FOR PLANT GROWTH FAILURE. THE SOILS TURN TO HARD, CEMENT-LIKE SUBSTANCES THAT DO NOT ALLOW ROOTS TO PENETRATE AND WATER TO DRAIN ADEQUATELY. 
DATE PLANTED: 28 DEC 93

$$
\begin{aligned}
& \text { HBG SOIL - PEAT/PERLITE MIX } \\
& \text { CONTROL }
\end{aligned}
$$

SAMPLE 1

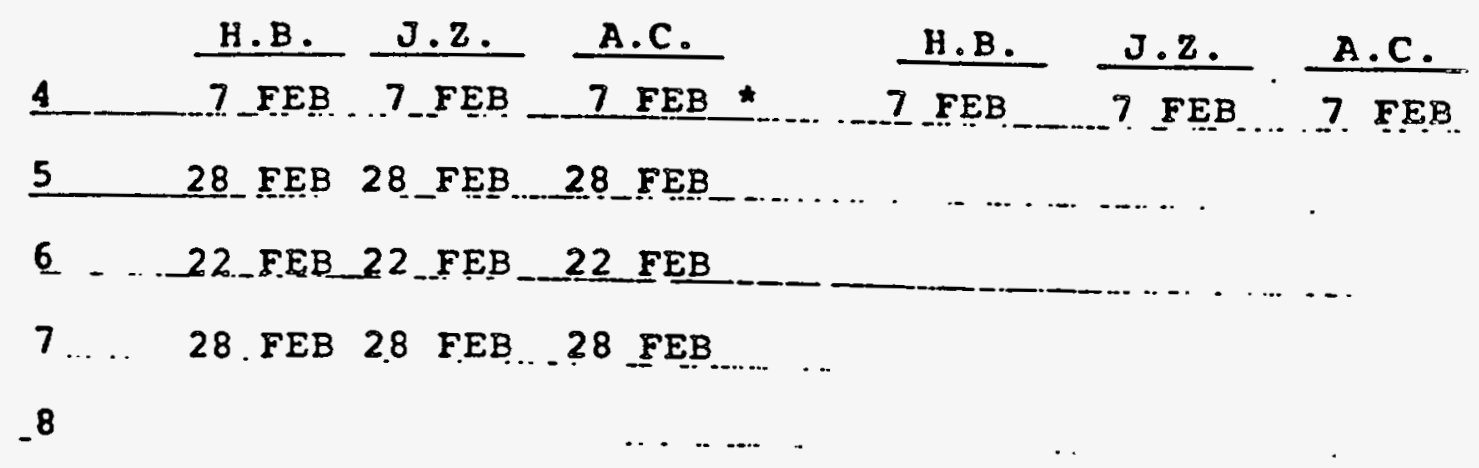

SAMPLE 2

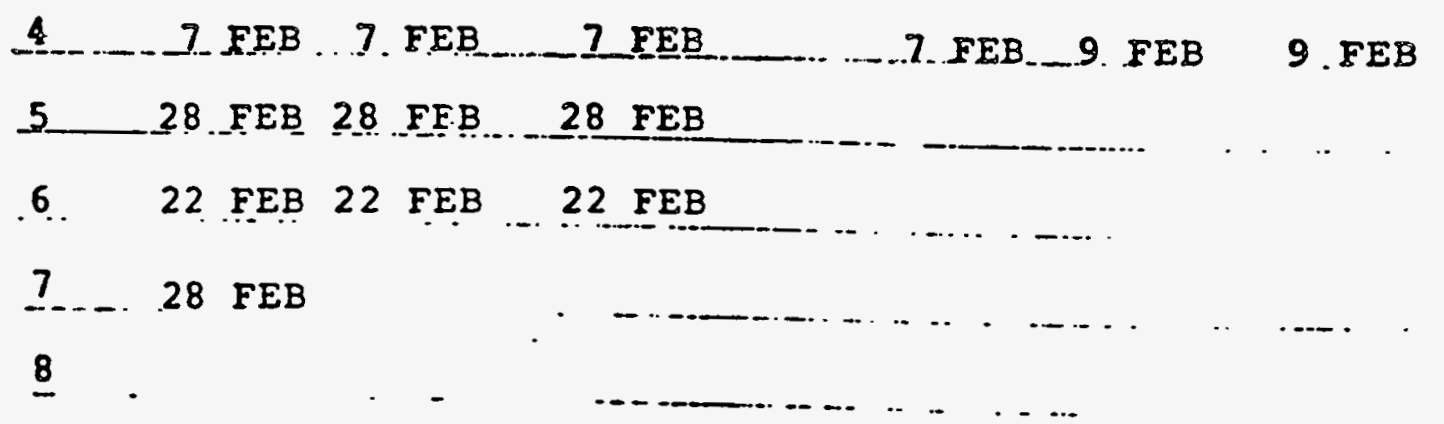

SAMPLE 3

4... 7 FEB 7 FEB 7 FEB $*$.... 7 FEB 9 FEB 9 FEB

$5 \quad 28$ FEB $\cdot 28$ FEB

622 FEB 22 FEB 22 FEB

728 FEB

$\underline{8}$

SAMPLE 4

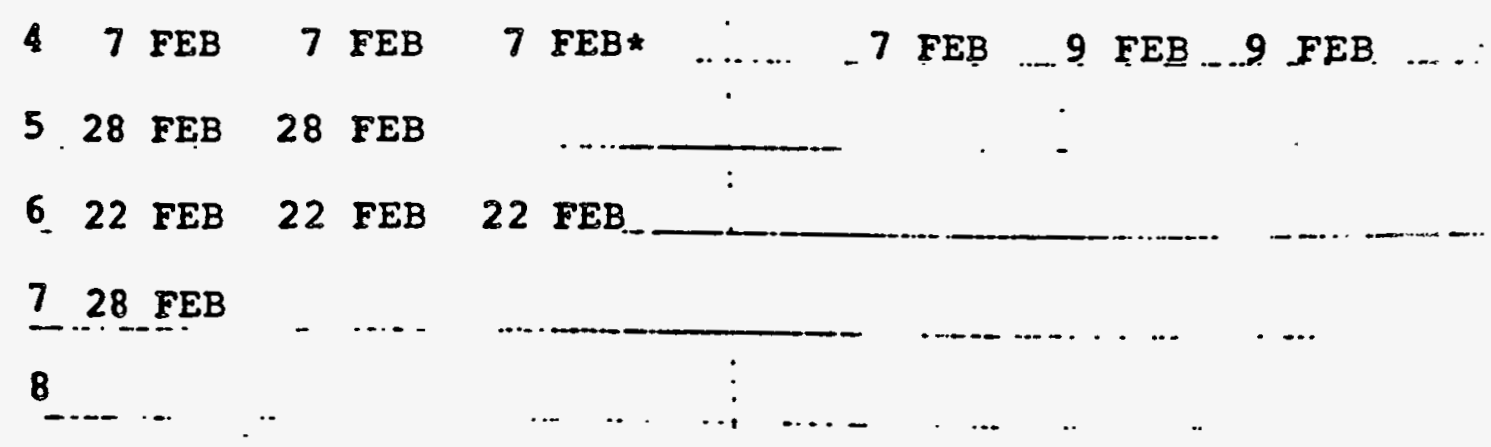

* Australian Carpet Grass 14 soil - Gonn growth occuxing

Notes: $2 / 28$ - Good growth - J.2. in 14 soil

$2 / 28$ - H.B. - starting to looked burnt.

$3 / 94$ - AIL POTS DEAD - YELLOWED AND/OR BURNY 
ASH UTILIZATION STUDY - H - POWER

SEEDS SOWN 4" POTS

DATE PLANTED: 6 JAN 94

CONTROL

SAMPLE 1

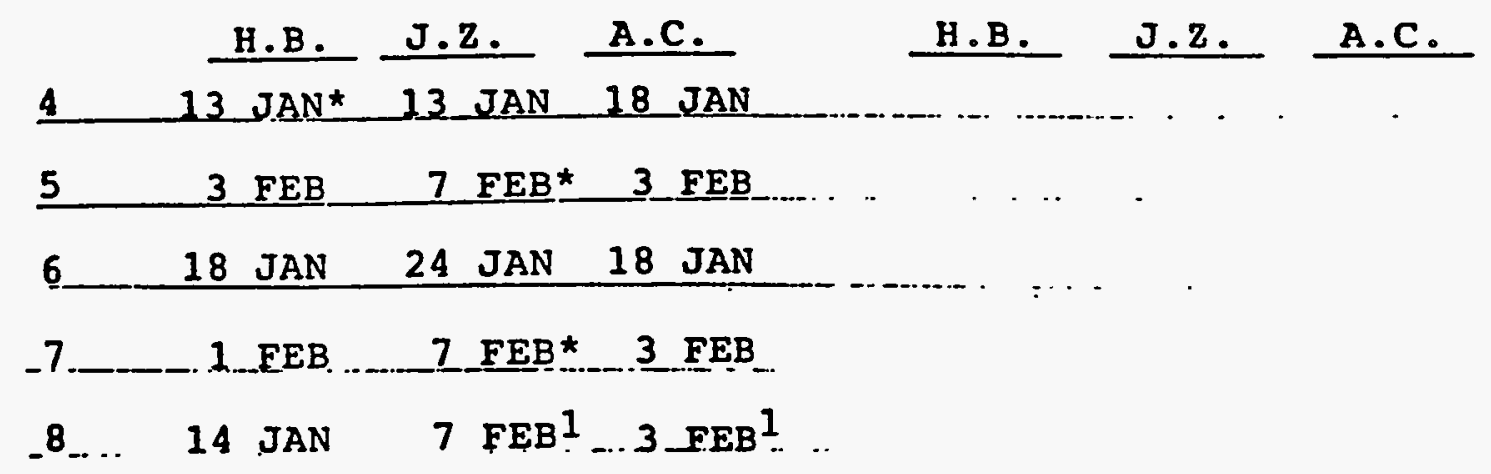

SAMPLE 2

\begin{tabular}{lllll}
-4 & $12 \mathrm{JAN}$ & 13 JAN 18 JAN \\
\hline 5 & 7 FEB & 7 FEB $^{*} 3$ FEB \\
\hline 6 & 24 JAN & 3 FEN 1 FEB \\
\hline 7 & 1 FEB .7 FEB & 3 FEB \\
\hline 8 & 14 JAN 7 FEB 117 FEB 1
\end{tabular}

SAMPLE 3

\begin{tabular}{|c|c|c|}
\hline 4 & 13 JAN & 14 JAN 18 JAN \\
\hline ..... & 7. .FEB & 9 FEB $\ldots . .3$ FEB \\
\hline 6 & $24 . J A N$ & 3. FEB \\
\hline 7 & 1 FEB & 7 FEB* \\
\hline 8 & 14 JAN & $7 \mathrm{FEB}^{1}$ \\
\hline
\end{tabular}

SAMPLE 4

\begin{tabular}{lrrrr}
4 & 13 JAN & 14 JAN & 18 JAN \\
5 & 16 JAN & 9 FEB & 16 FEB \\
\hline 6 & 7 FEB & 3 FEB & 1 FEB \\
\hline 7 & 1 FEB & 9 FEB & 7 FEB \\
\hline 8 & 3 FEB & $=$ & $\ldots$ & - \\
\hline & * Sickly & - FEB 28 \\
1 Dead & - FEB 28 \\
$3 / 14 / 94$ & - All dead
\end{tabular}




\section{Turfgrass}
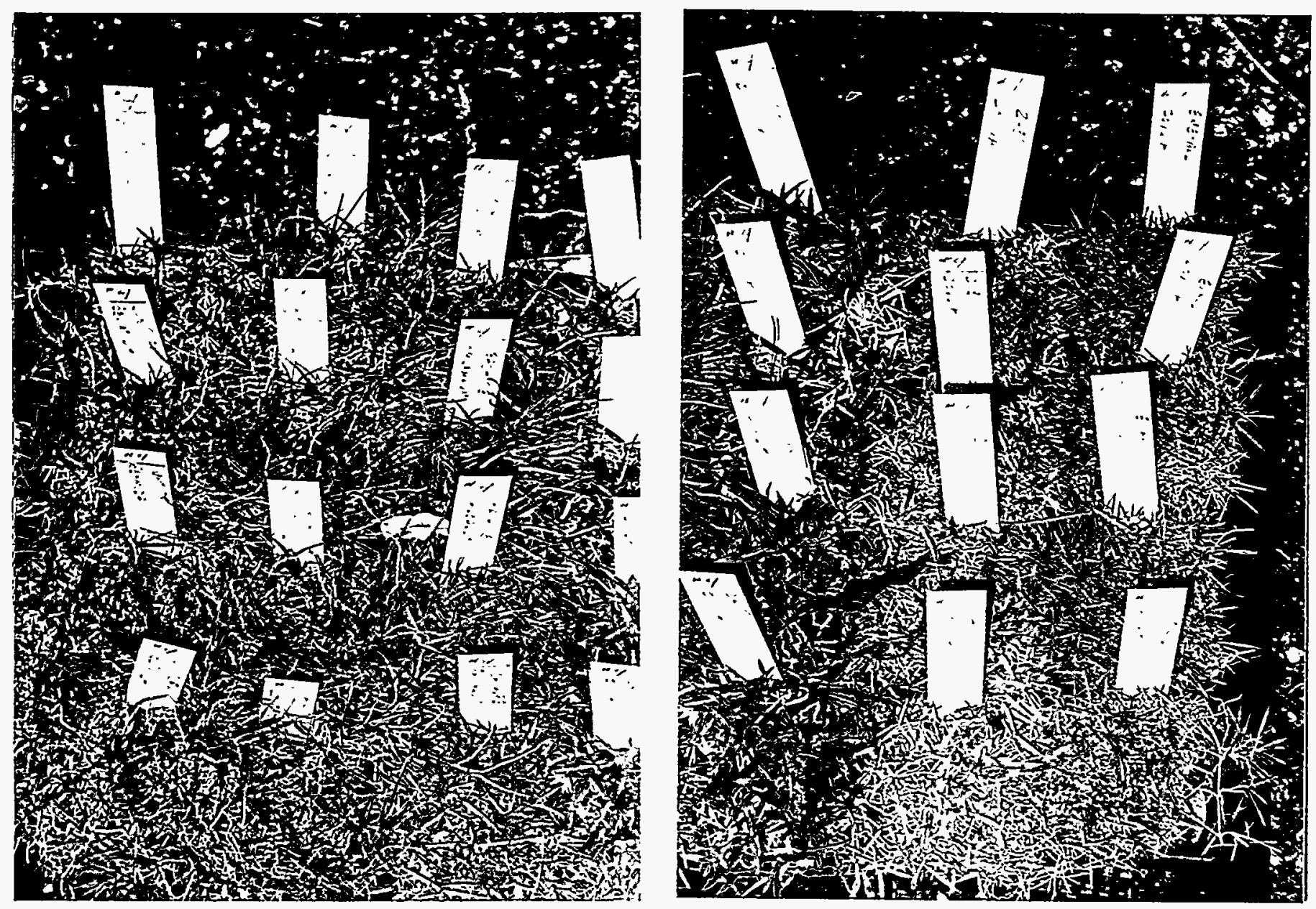

Mixture \#4 Photographed January 28, 1994 

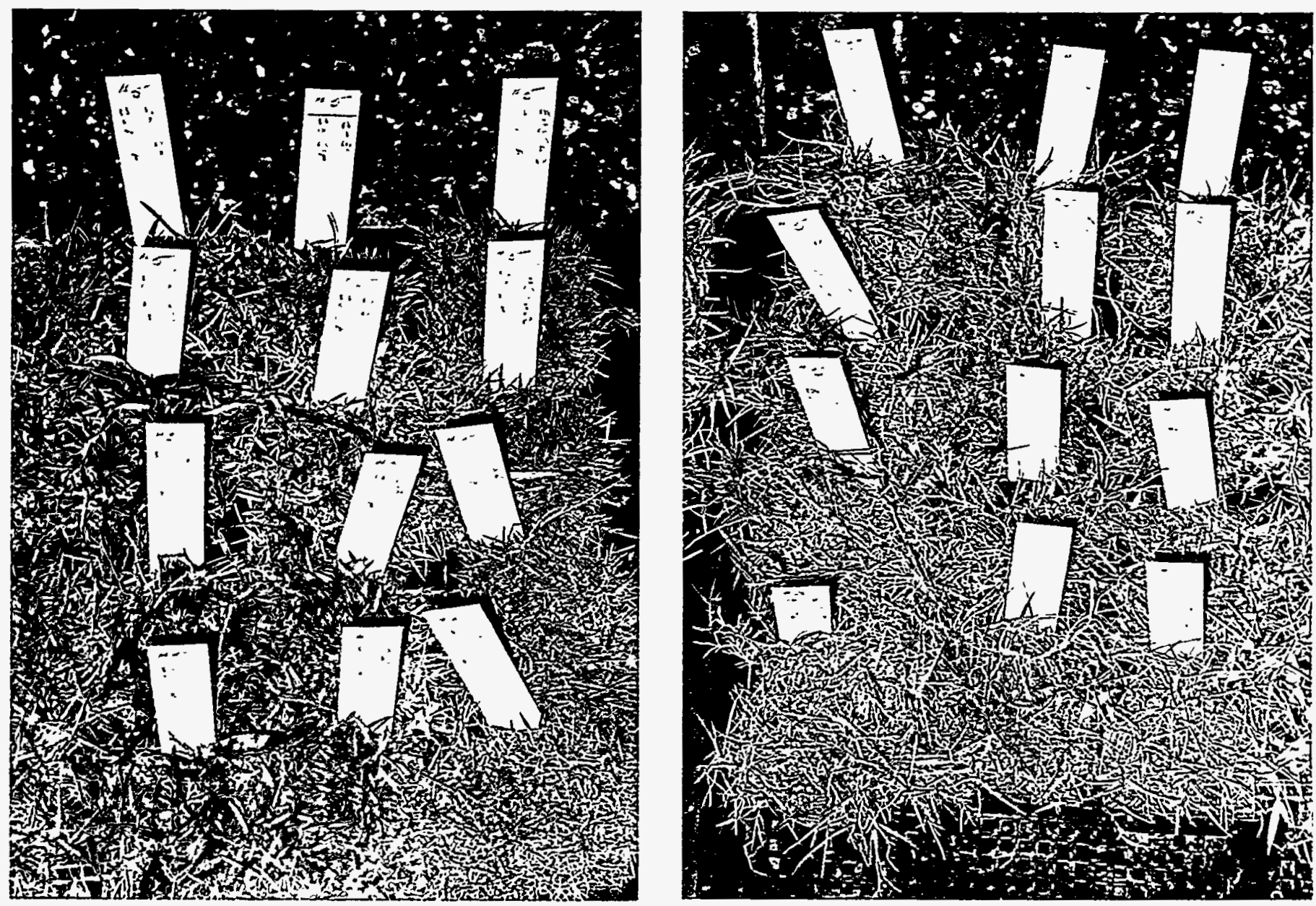

Mixture \#5 Photographed January 28, 1994 


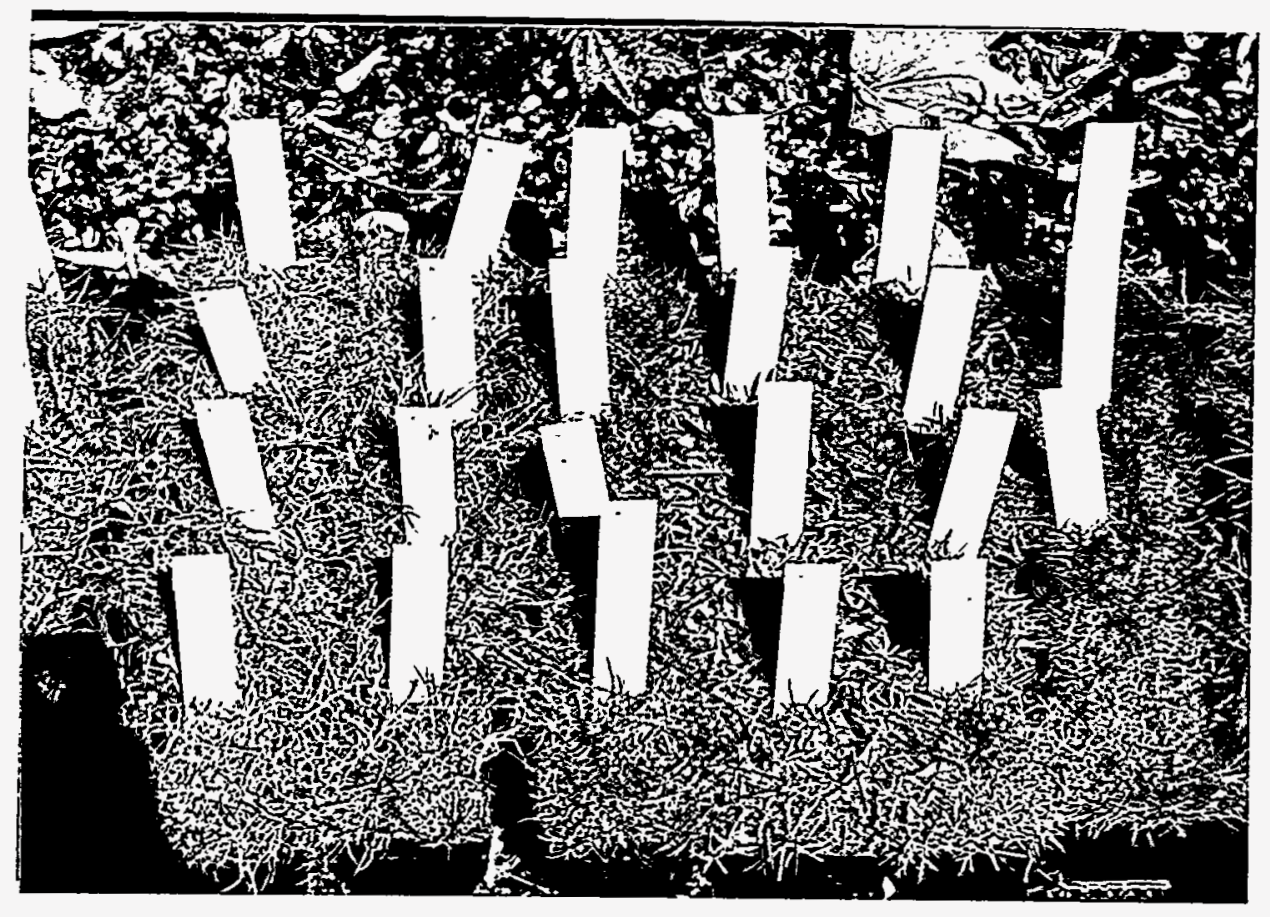

Mixture \#5 Photographed February 28, 1994 

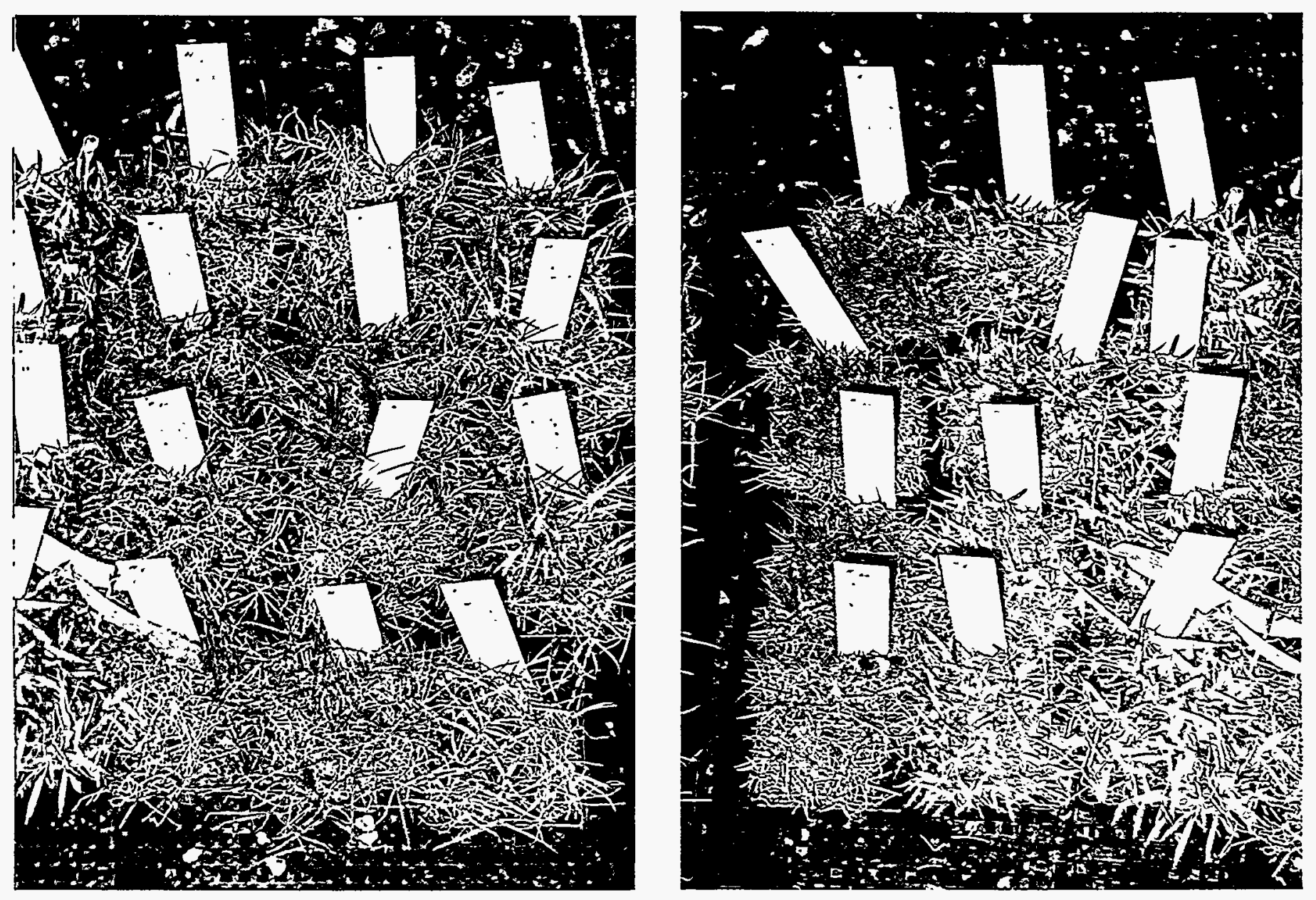

Mixture \#6 Photographed January 28, 1994 


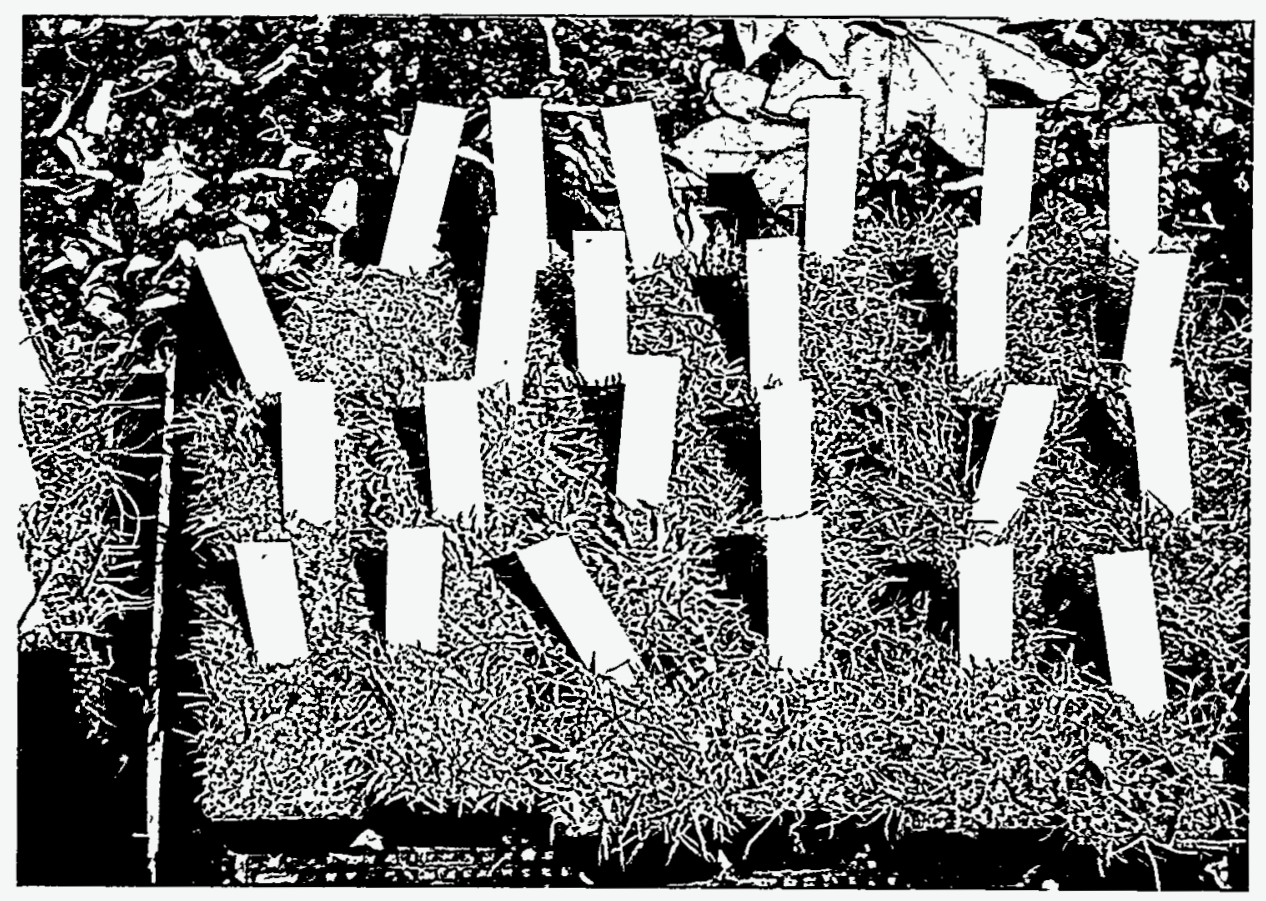

Mixture \#6 Photographed February 28, 1994 

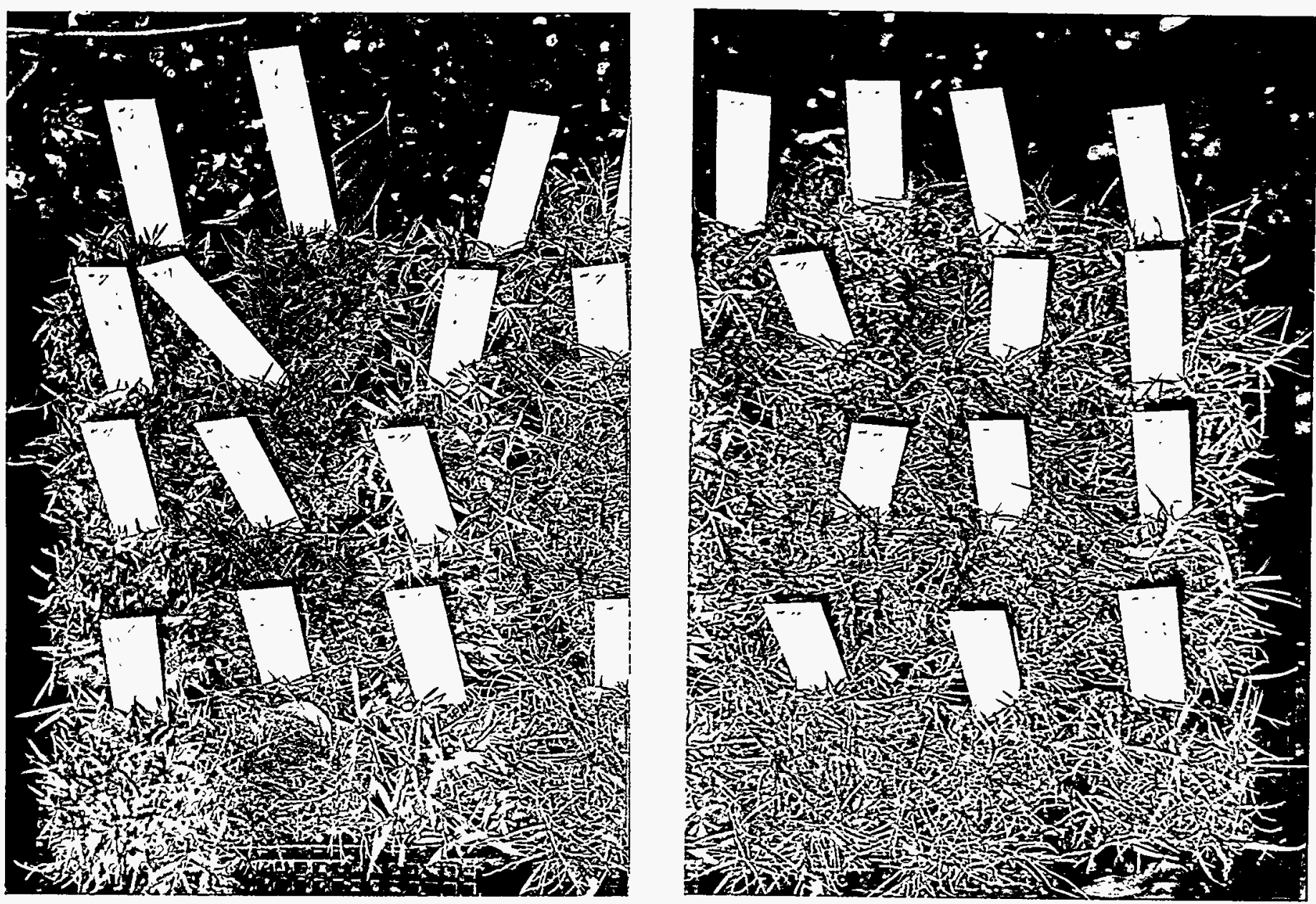

Mixture \#7 Photographed January 28, 1994 


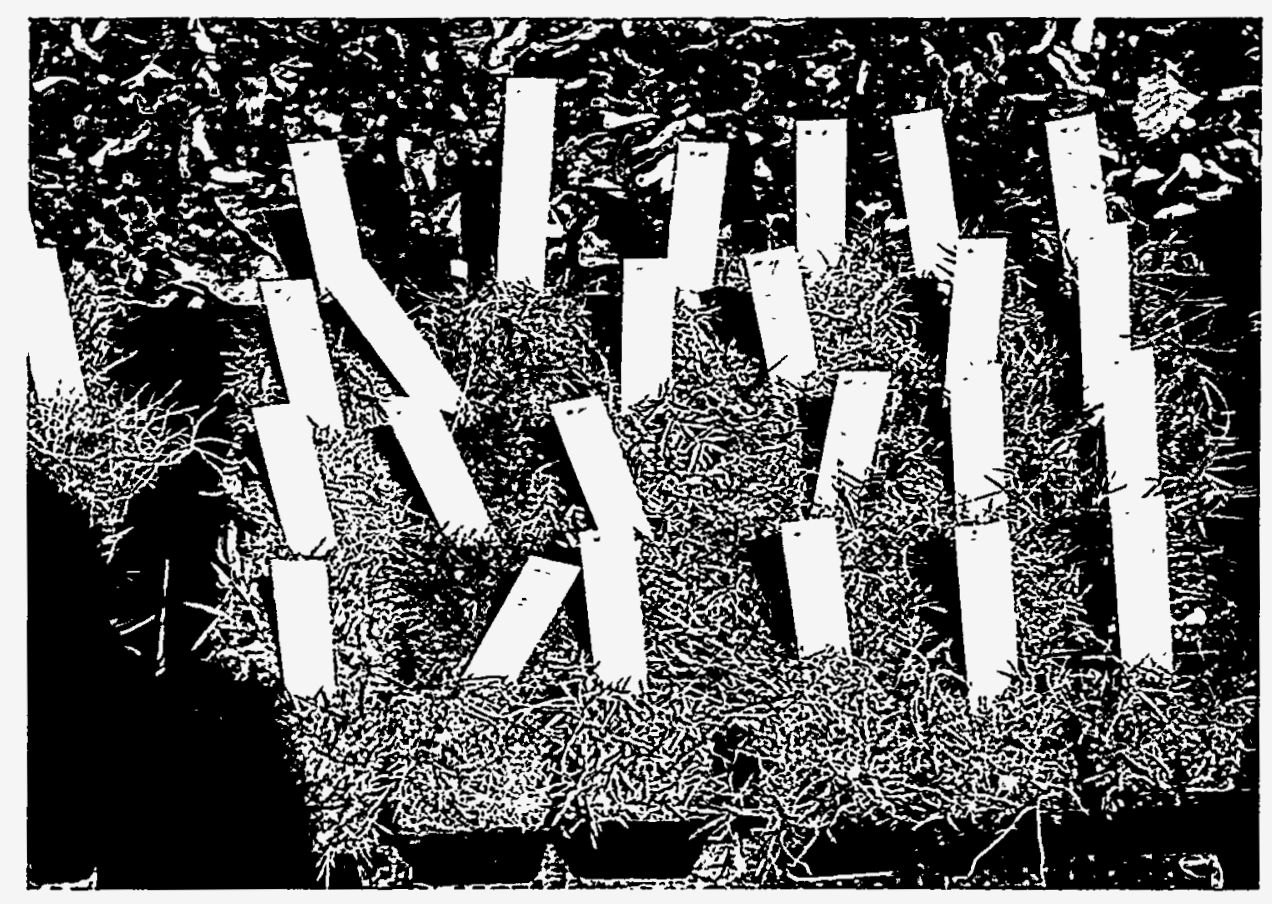

Mixture \#7 Photographed February 28, 1994 

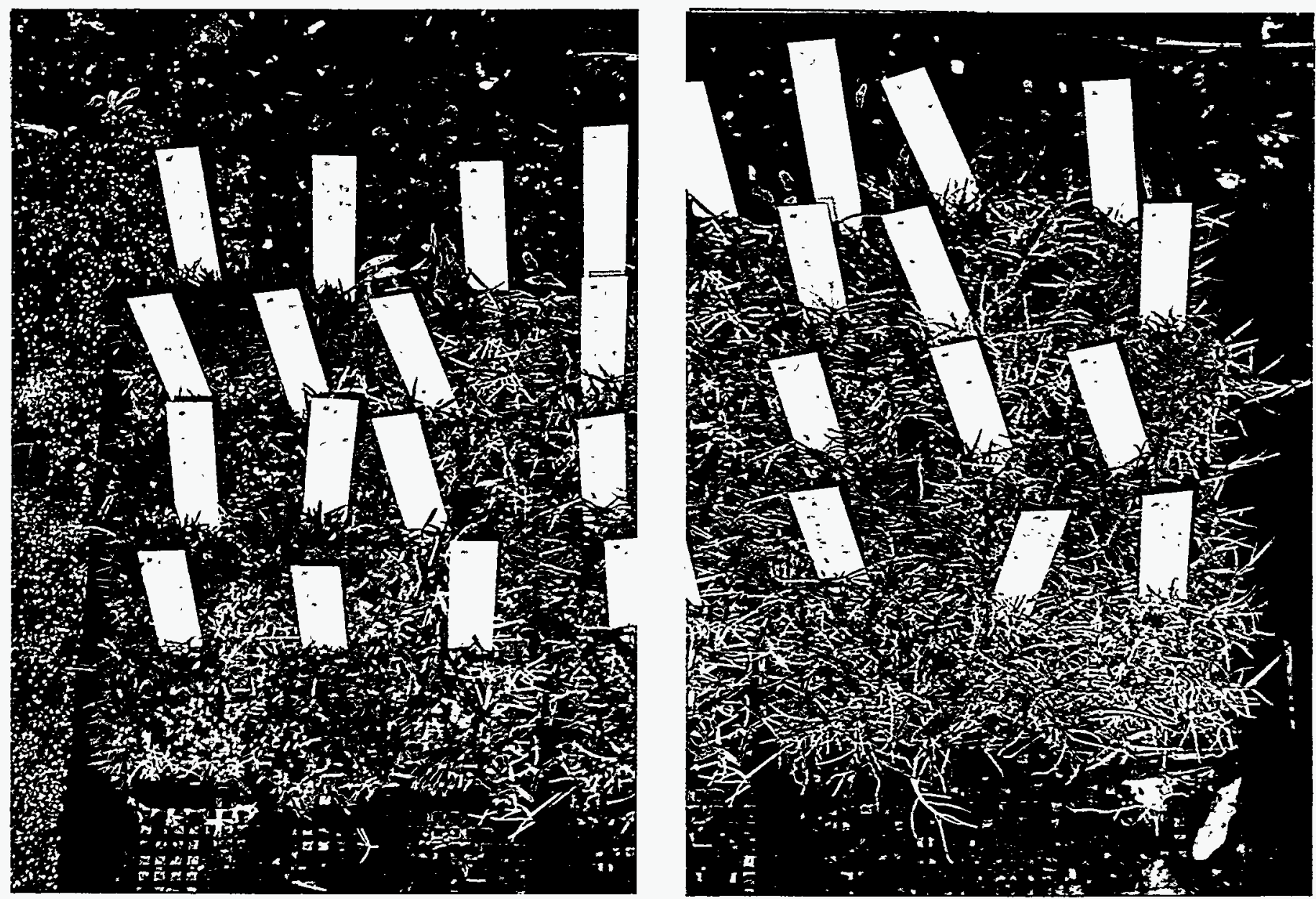

Mixture \#8 Photographed January 28, 1994 


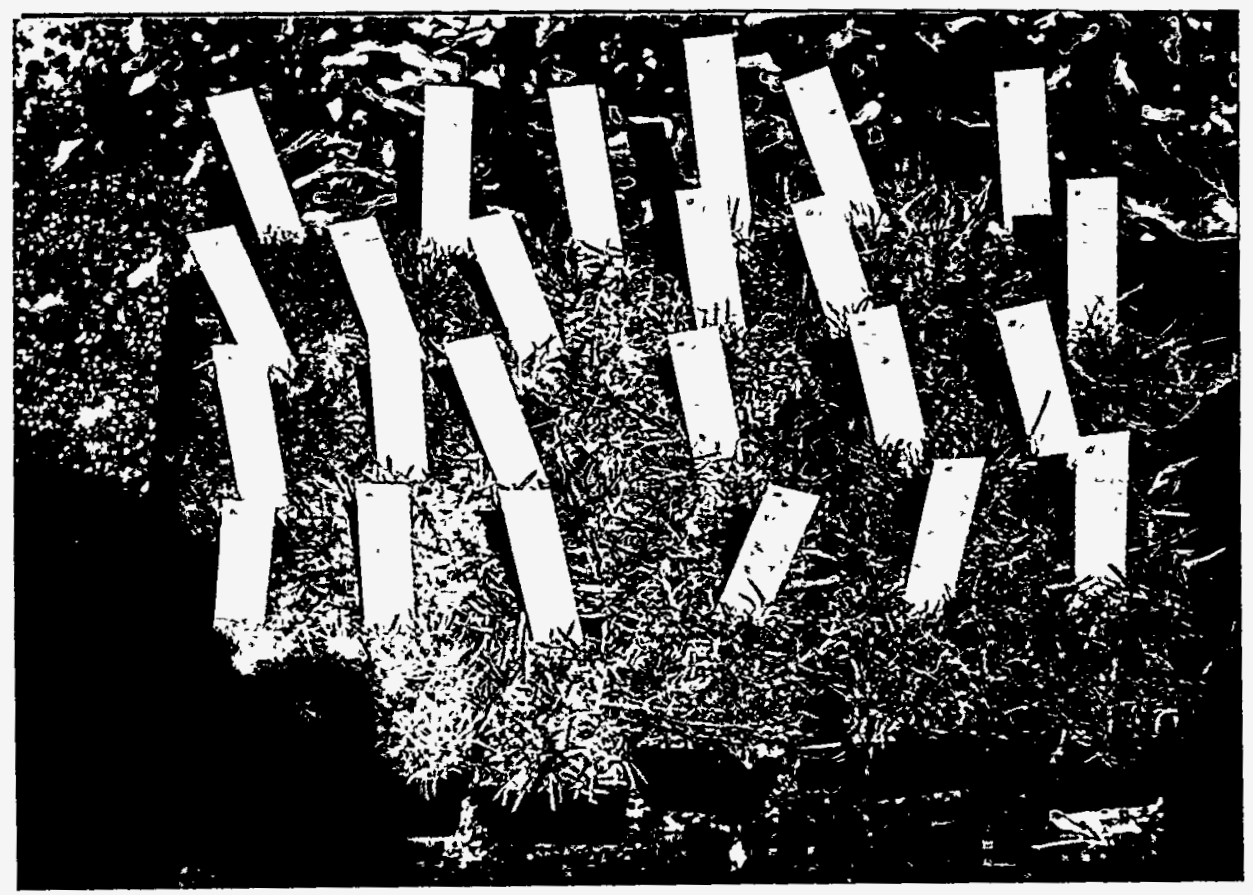

Mixture \#8 Photographed February 28, 1994 


\section{Seedlings}

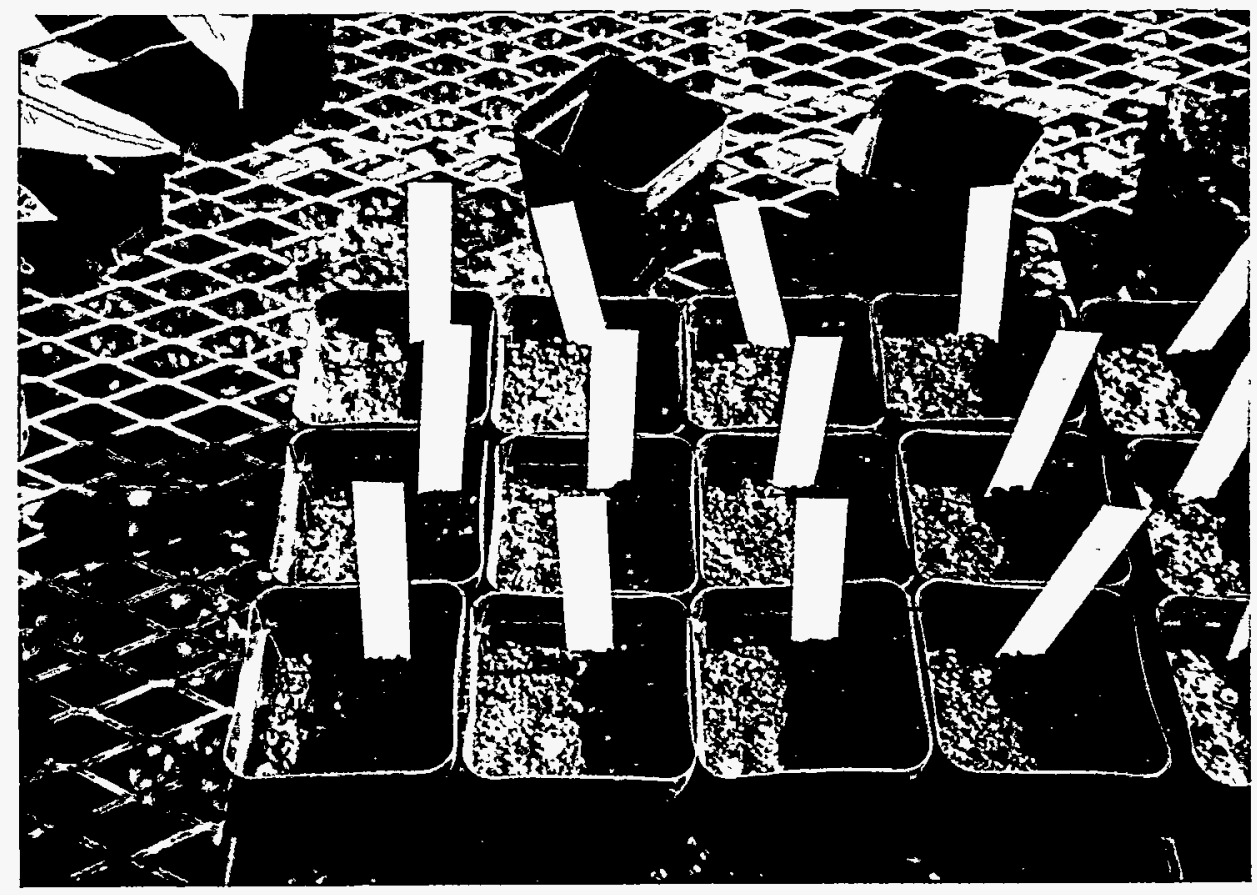

Mixture \#4 Photographed January 28, 1994 

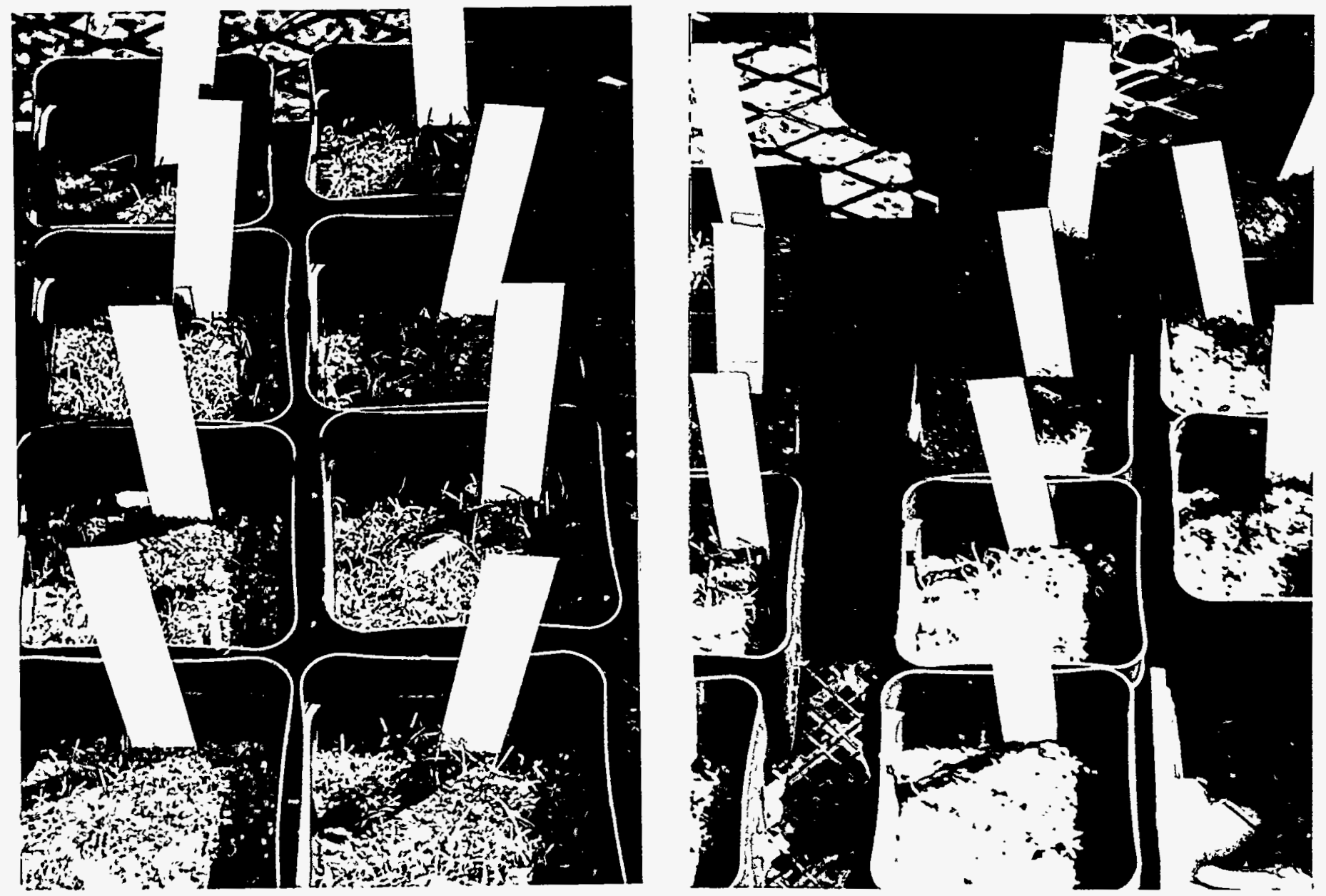

Mixture \#4 Photographed February 28, 1994 


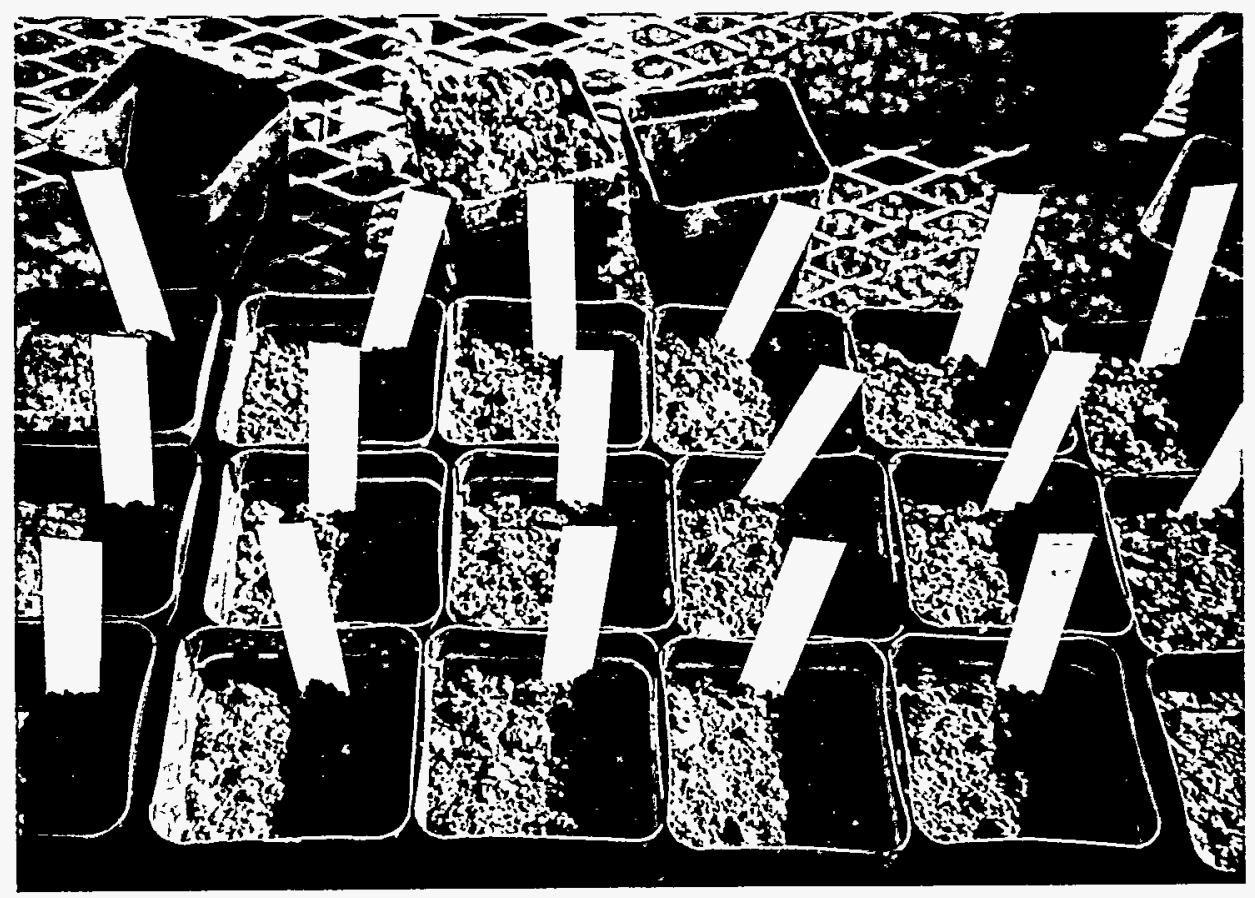

Mixture \#5 Photographed January 28, 1994 

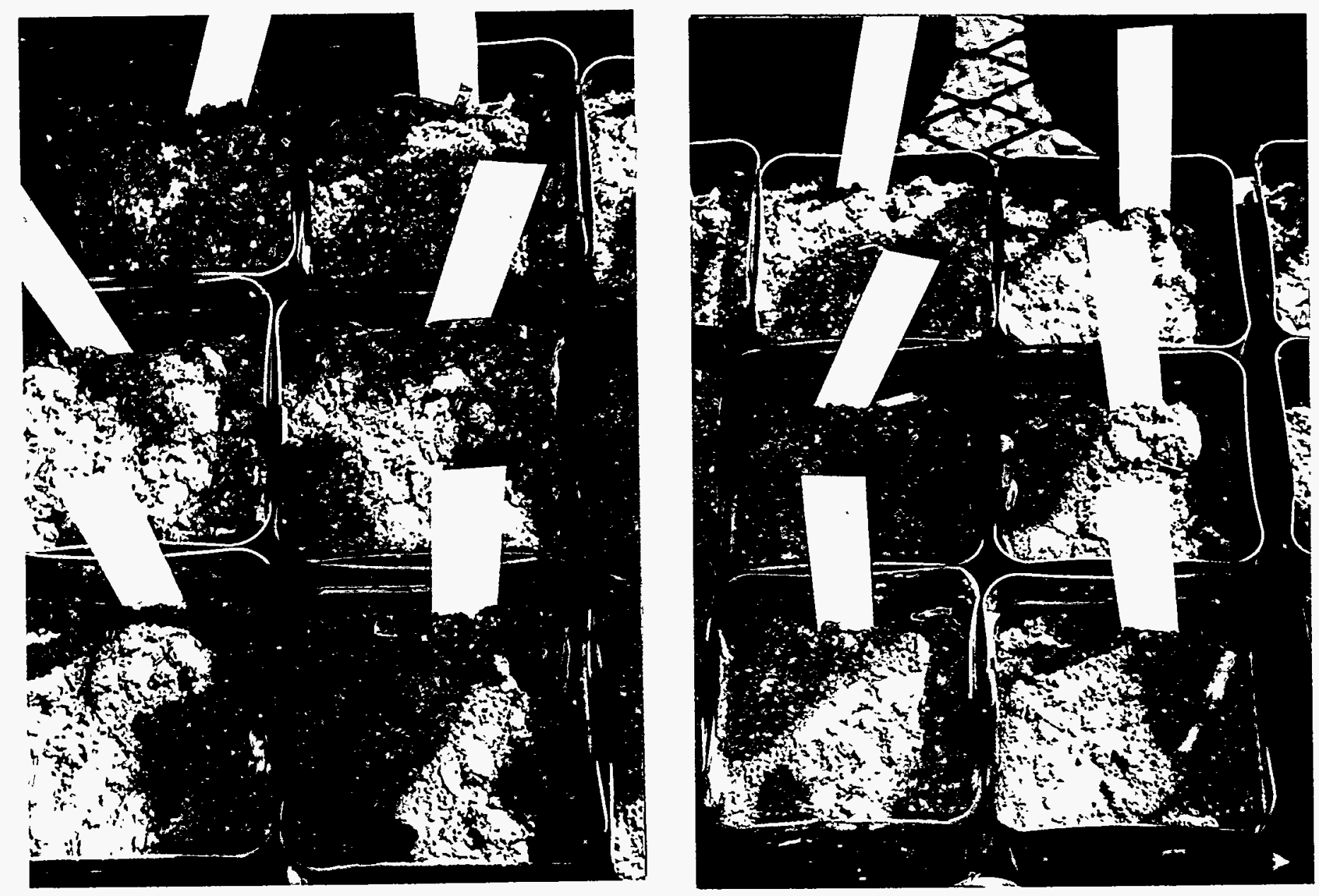

Mixture \#5 Photographed February 28, 1994 


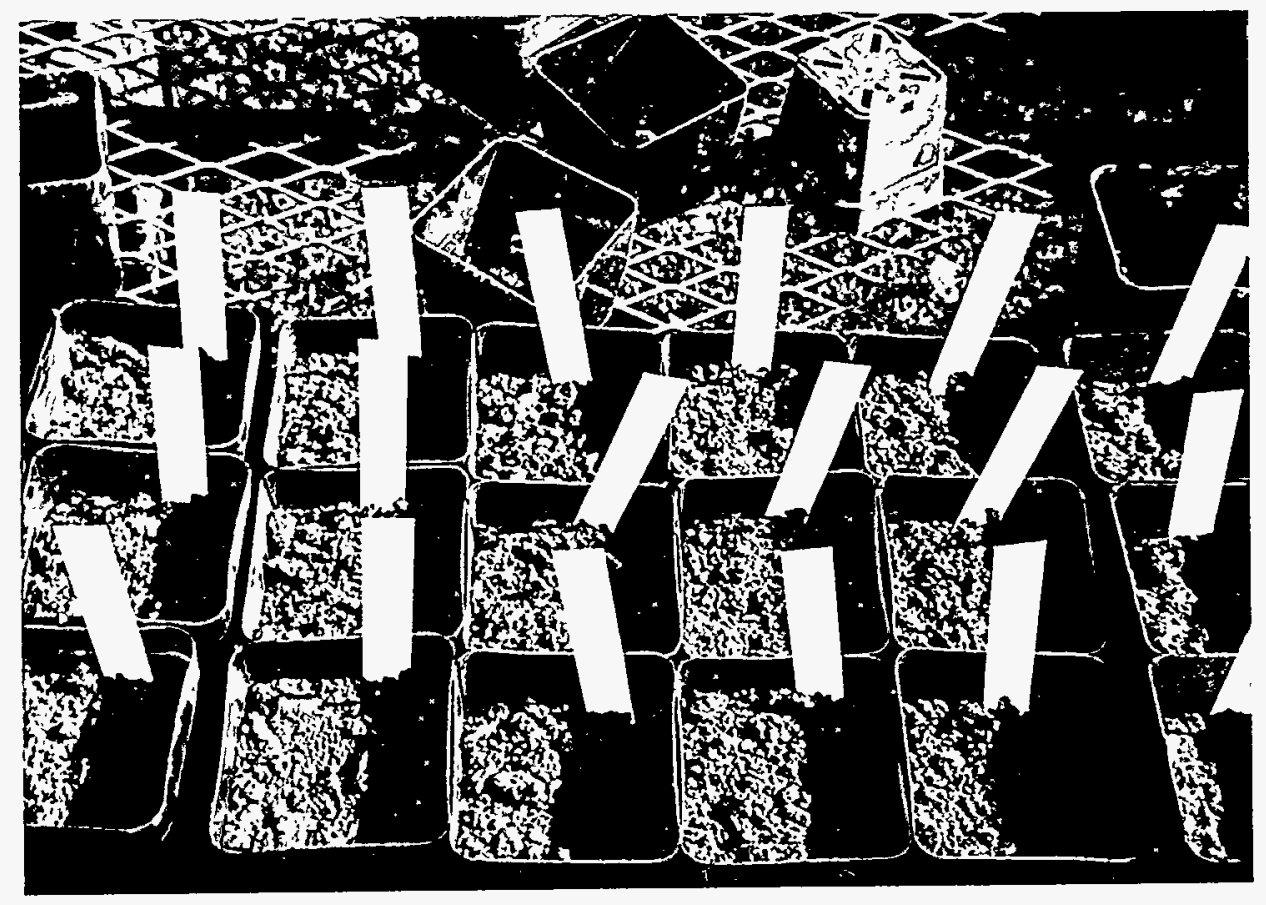

Mixture \#6 Photographed January 28, 1994 


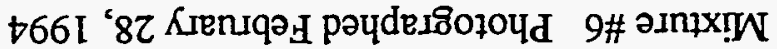
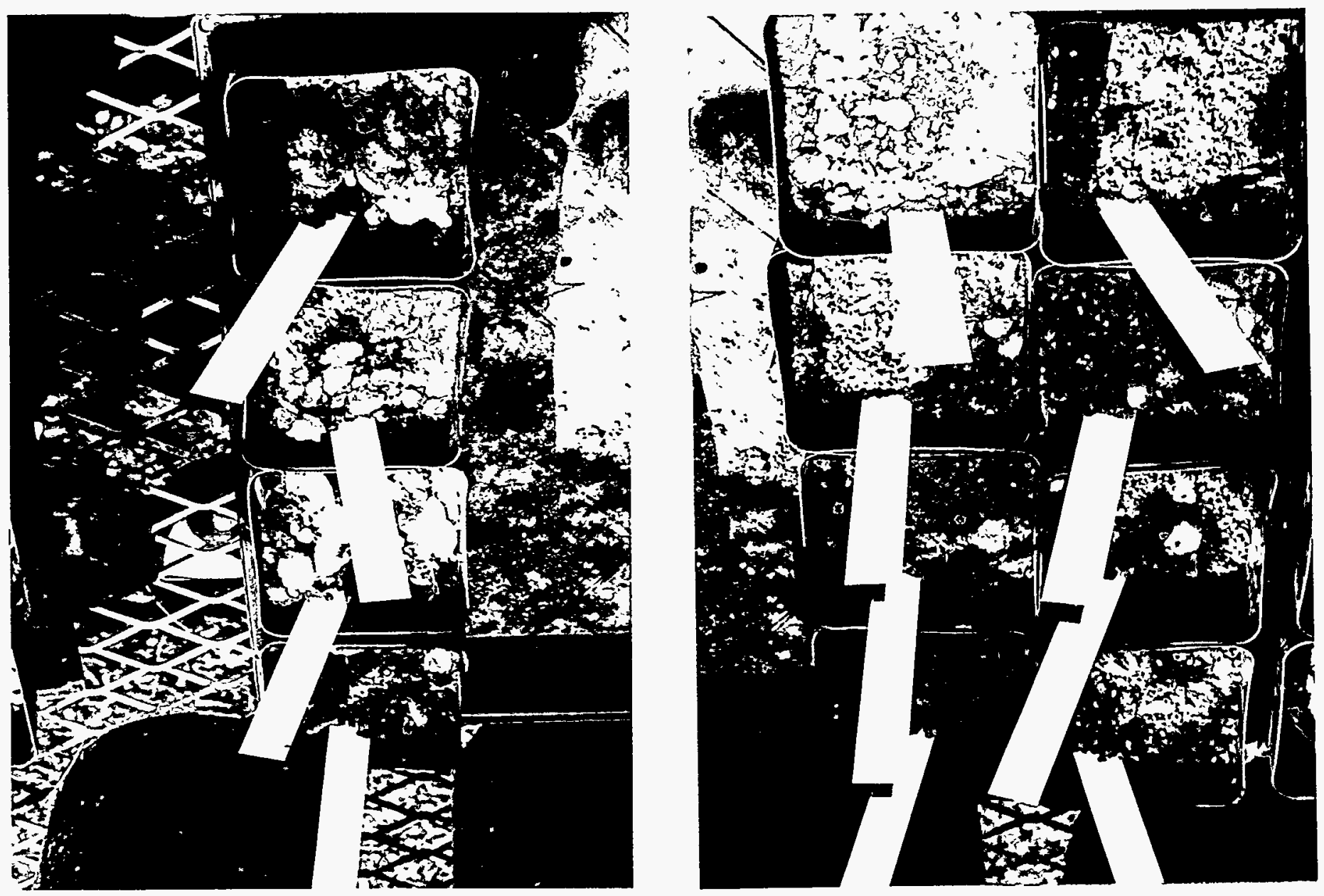
†66I '8Z Krenue[ pәчdes80

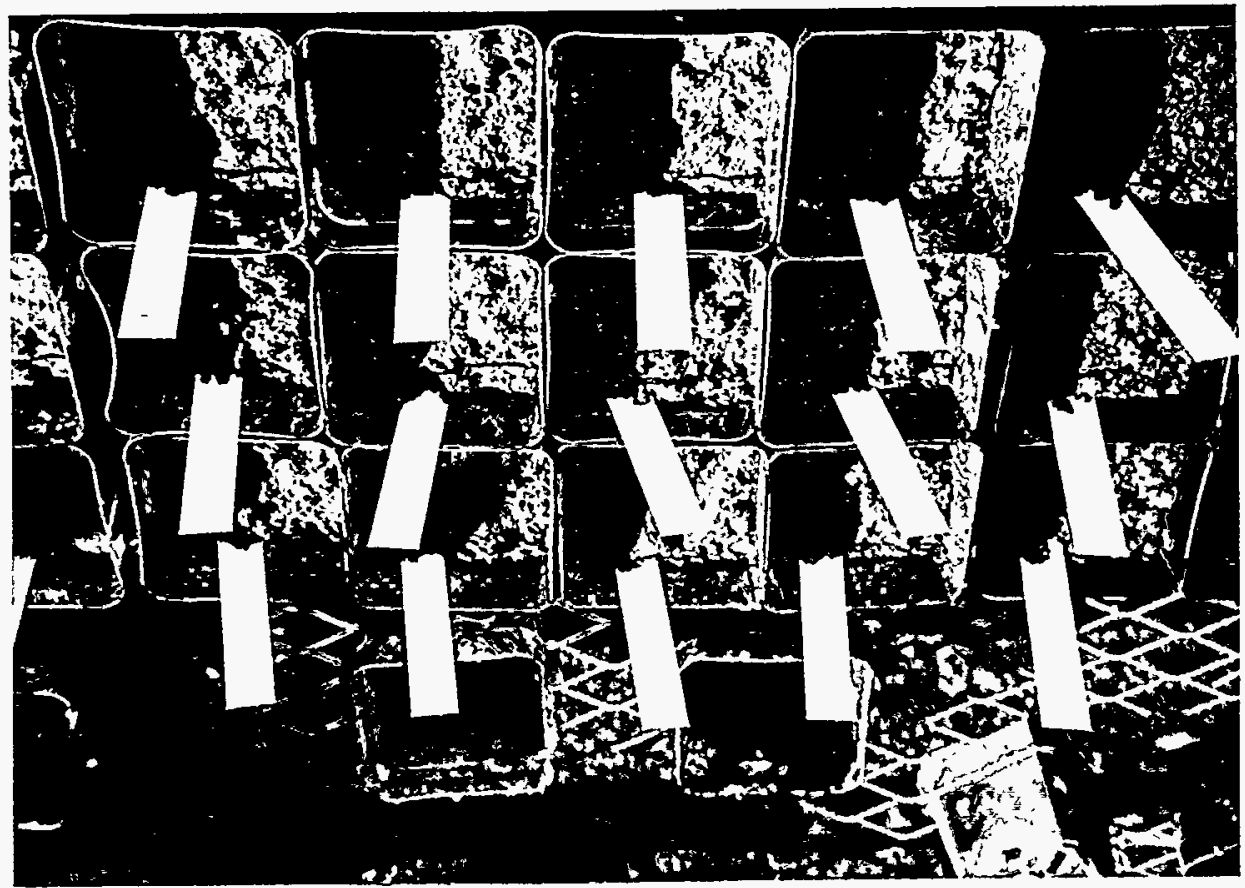



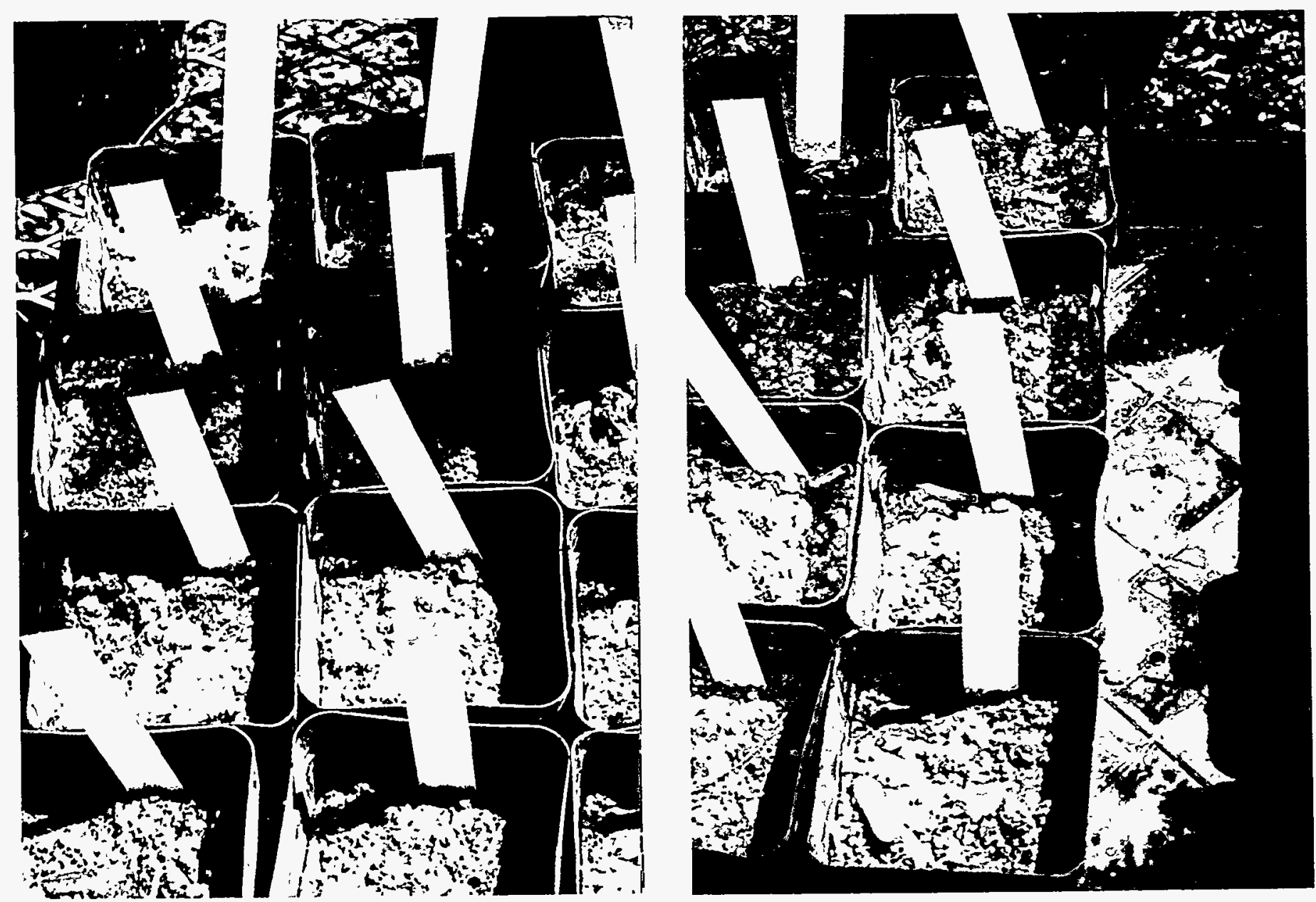

Mixture \#7 Photographed February 28, 1994 


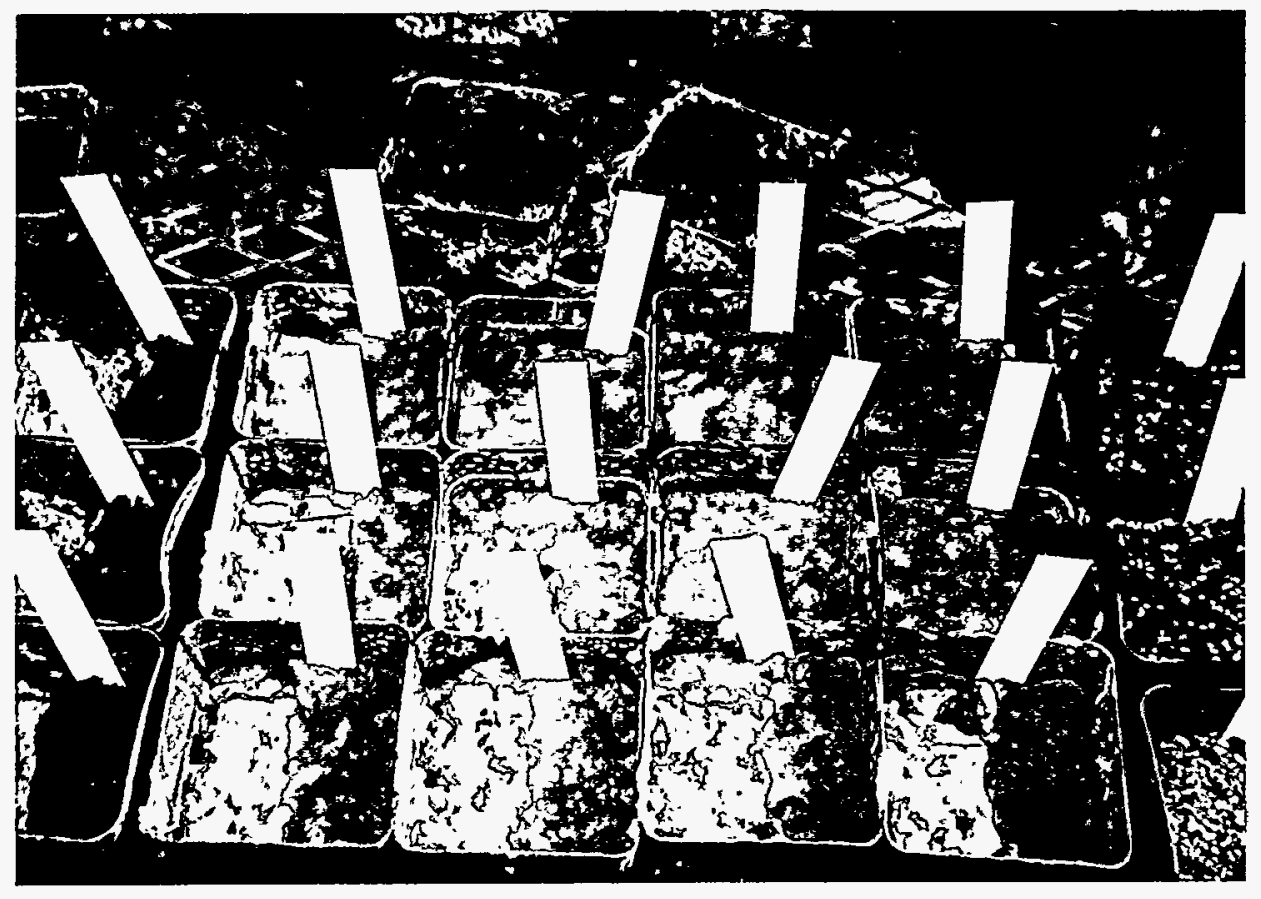

Mixture \#8 Photographed January 28, 1994 

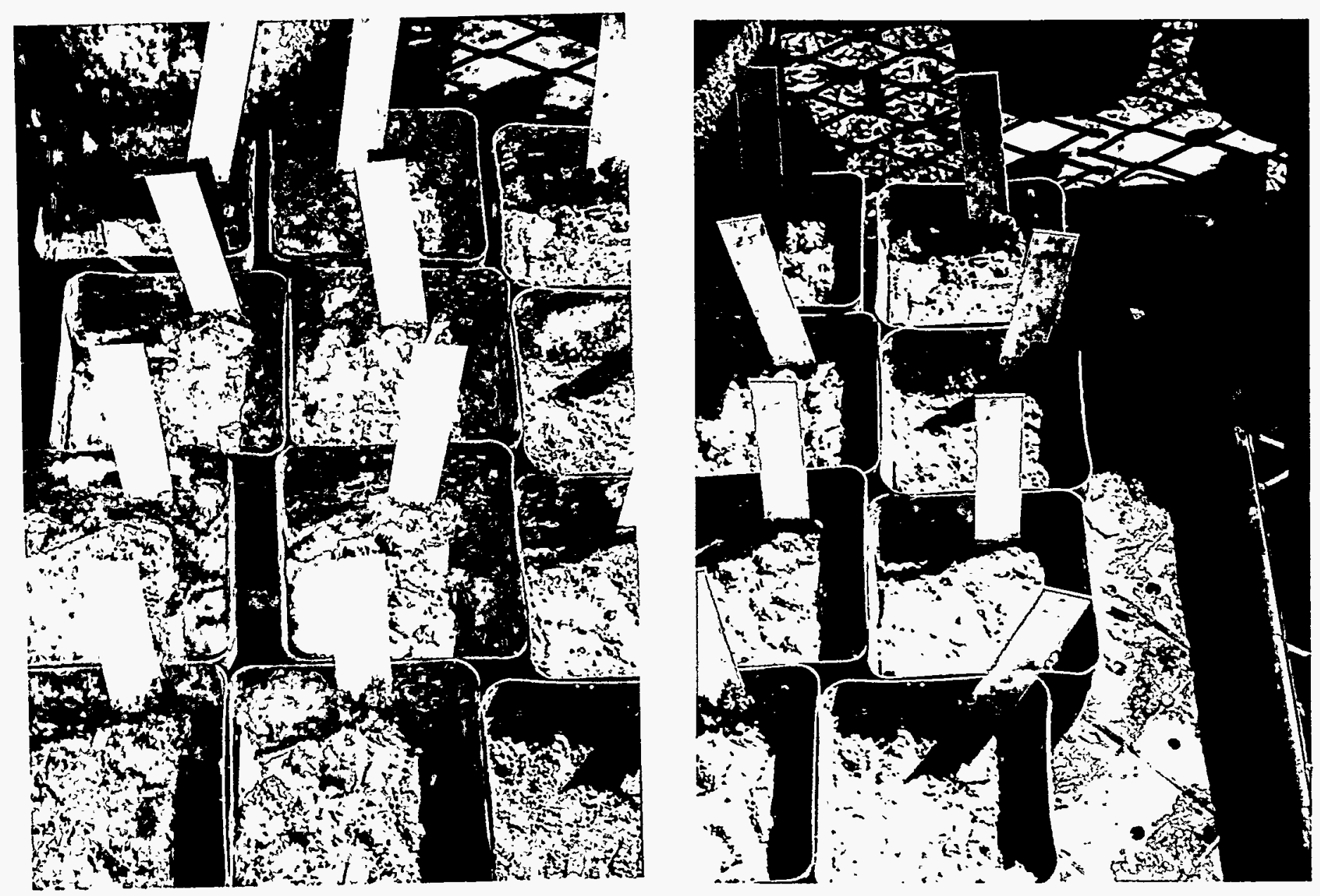

Mixture \#8 Photographed February 28, 1994 
Appendix A6

Erosion Test

\section{Observations}

During the early stages, there was no visible erosion in any of the ash mixtures and only two exhibited signs of cracking: mixtures \#1 and \#4. Although none of the mixtures containing sludge exhibited any signs of cracking, they did undergo shrinkage which was evident from the voids appearing around the edge of the box during curing. It is hypothesized that this is largely due to the fiber-like qualities of the sludge. It is also believed to be the same reason why the sludge mixtures possessed some flexibility while also exhibiting cement-like properties.

With time, mixtures \#1, \#2, and \#4, showed gradual signs of wind and rain erosion. Mixtures \#2 and \#4 were characteristically loose in nature and were easily disturbed by the elements. However, the cracks in mixture \#1 grew in size and severity causing some fragmentation and subsequent erosion of the fine fly ash. Rust developed on the ferrous material in mixture \#9 and subsequently, in mixtures \#2 and \#3. Eventually, the rust in mixtures \#3 and \#9 led to the formation of small cracks and also consequently caused some fragmentation and erosion.

During the month of February, considerable winds and rain were experienced, which dramatically affected most of the test mixtures--especially those containing sludge. The frequent heavy downpours caused their surface to flake and erode towards the low end of the box. The degree of damage sustained varied from minor flaking, as in mixture \#6, to formation of grooves about $0.6 \mathrm{~cm}$. in depth, as in mixtures \#5 and \#7. Mixtures \#5 and \#7 seemed to have a much higher capacity to retain moisture than any other mixture which was believed to be due to the high percentage of sludge. Mixture \#8 seems to have been the only test mixture unaffected by the rain. This mixture behaved almost like concrete, and remained hard and durable. Table 1 on the following page summarizes the mixtures' performance for the three test criteria.

Selected photographs of the mixtures taken during the test period, appear beginning on page A6-3. 


\begin{tabular}{|c|c|c|c|}
\hline Mixtures & $\begin{array}{c}\text { Erosion } \\
\text { Resistance } \\
\text { (least - most) }\end{array}$ & $\begin{array}{c}\text { Material } \\
\text { Strength } \\
\text { (soft - hard) }\end{array}$ & $\begin{array}{c}\text { Shrinkage } \\
\text { (least - most) }\end{array}$ \\
\hline Mix 1 & 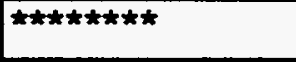 & $\star \star \star \star \star \star \star$ & $\star \star \star \star \star \star \star \star$ \\
\hline Mix 2 & $\star \star \star \star$ & $\star \star$ & $n / a$ \\
\hline Mix 3 & $\star \star \star \star \star$ & $\overline{\hbar \star \star \star \star \star \star \star \star \star \star \star}$ & $\overline{\star \star \star \star \star \star \star \star \star \star \star}$ \\
\hline Mix 4 & $\star \star$ & $\star \star$ & $n / a$ \\
\hline Mix 5 & $\star \star \star \star \star \star \star$ & $\star \star \star \star \star \star$ & 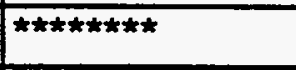 \\
\hline Mix 6 & 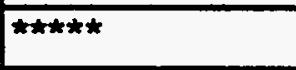 & 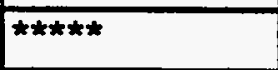 & 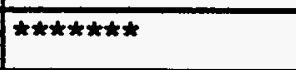 \\
\hline Mix 7 & $\star \star \star \star \star \star$ & $\star \star \star \star$ & $\star \star \star \star \star$ \\
\hline Mix 8 & $\overline{\star \star \star \star \star \star \star \star \star \star \star}$ & 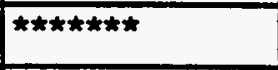 & $\star \star \star$ \\
\hline $\operatorname{Mix} 9$ & $\star \star \star \star \star$ & 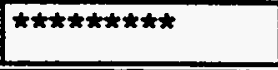 & 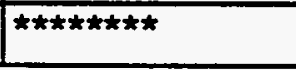 \\
\hline
\end{tabular}

Table 1

\section{Conclusions}

Overall, the mixtures containing fly ash fared quite well, and there was no single mixture which was clearly better than the rest. However, the economics of the mixtures must also be taken into account, if large scale applications are to be a reality. Among the mixtures which held up the best $(1,3,5,6,7,8$, and 9), only one was truly economical: mixture \#3. This mixture represents the entire ash stream produced by H-POWER, requires no additional processing, no stabilizing additives, and no mixing. Accordingly, mixture \#3 was chosen as the top performer for this subtask. 

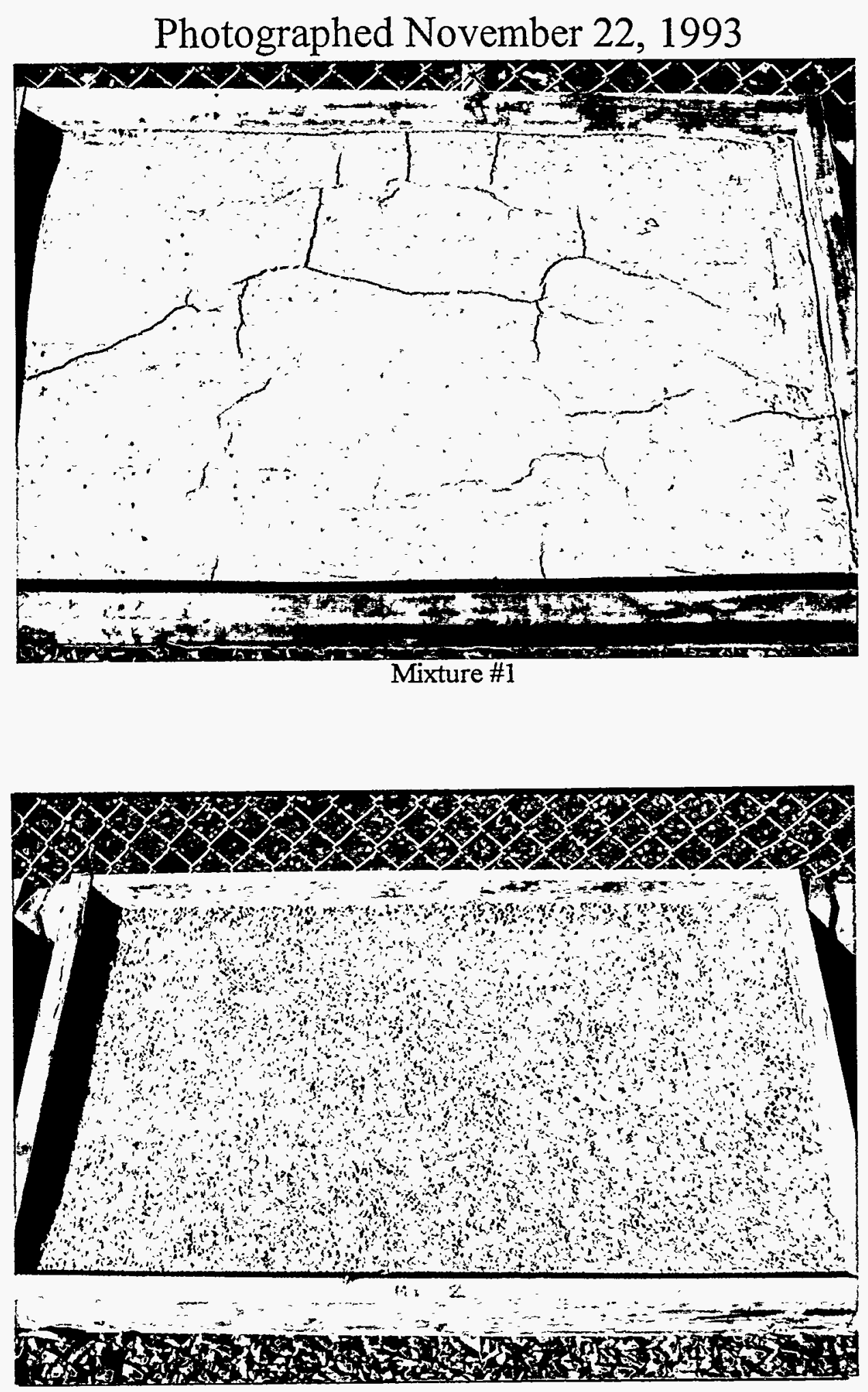

Mixture \#2 

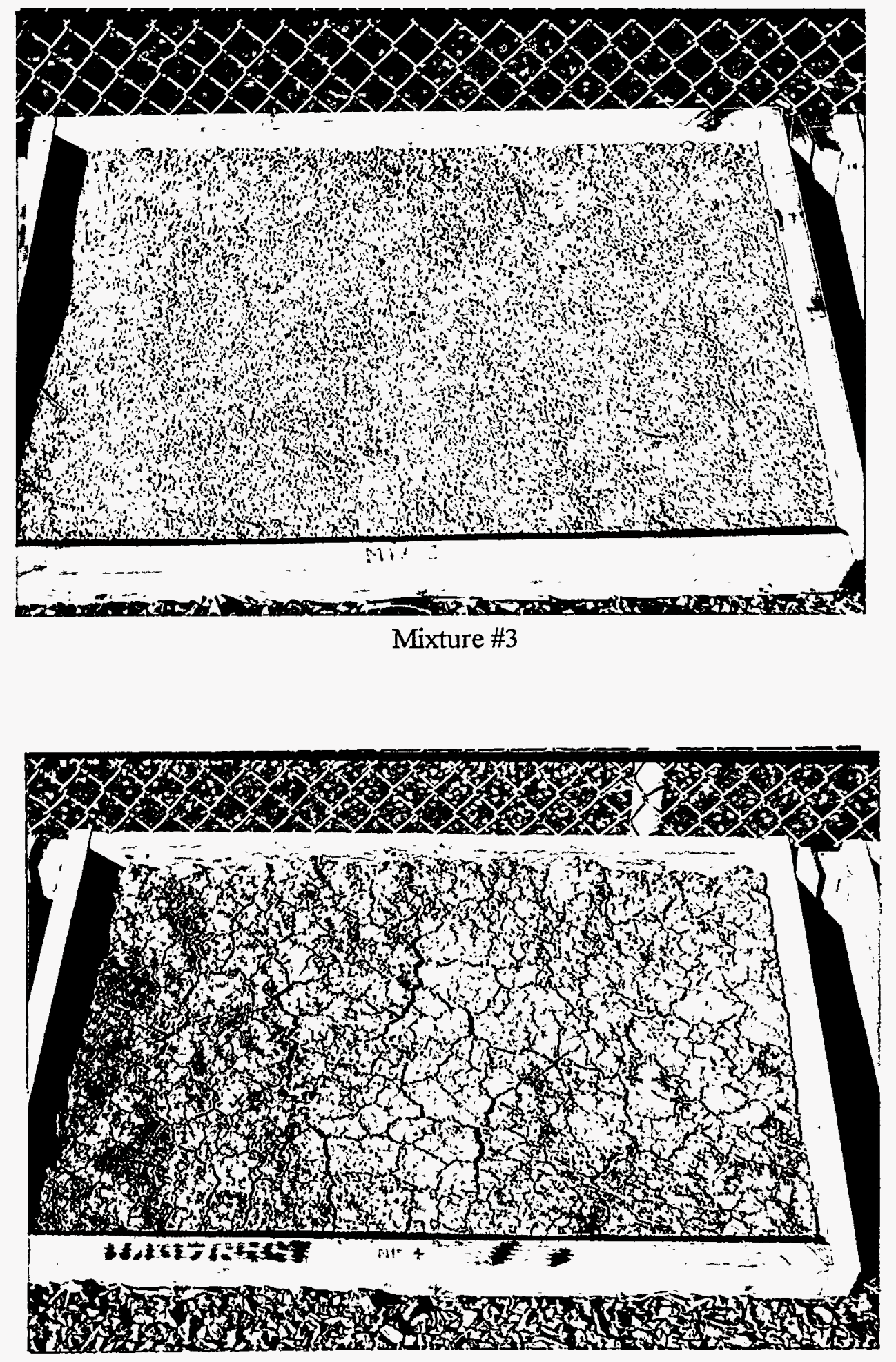

Mixture \#4 


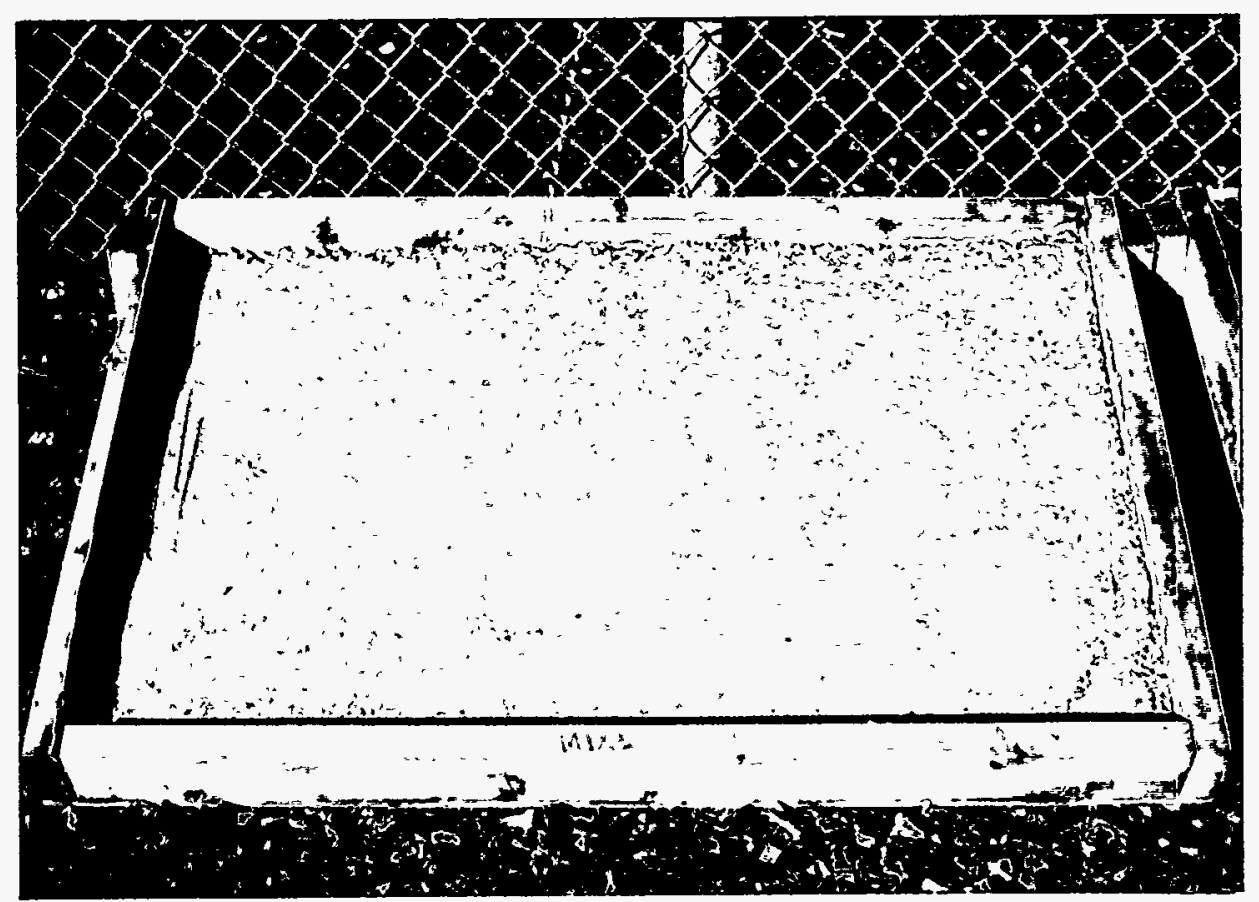

Mixture \#5

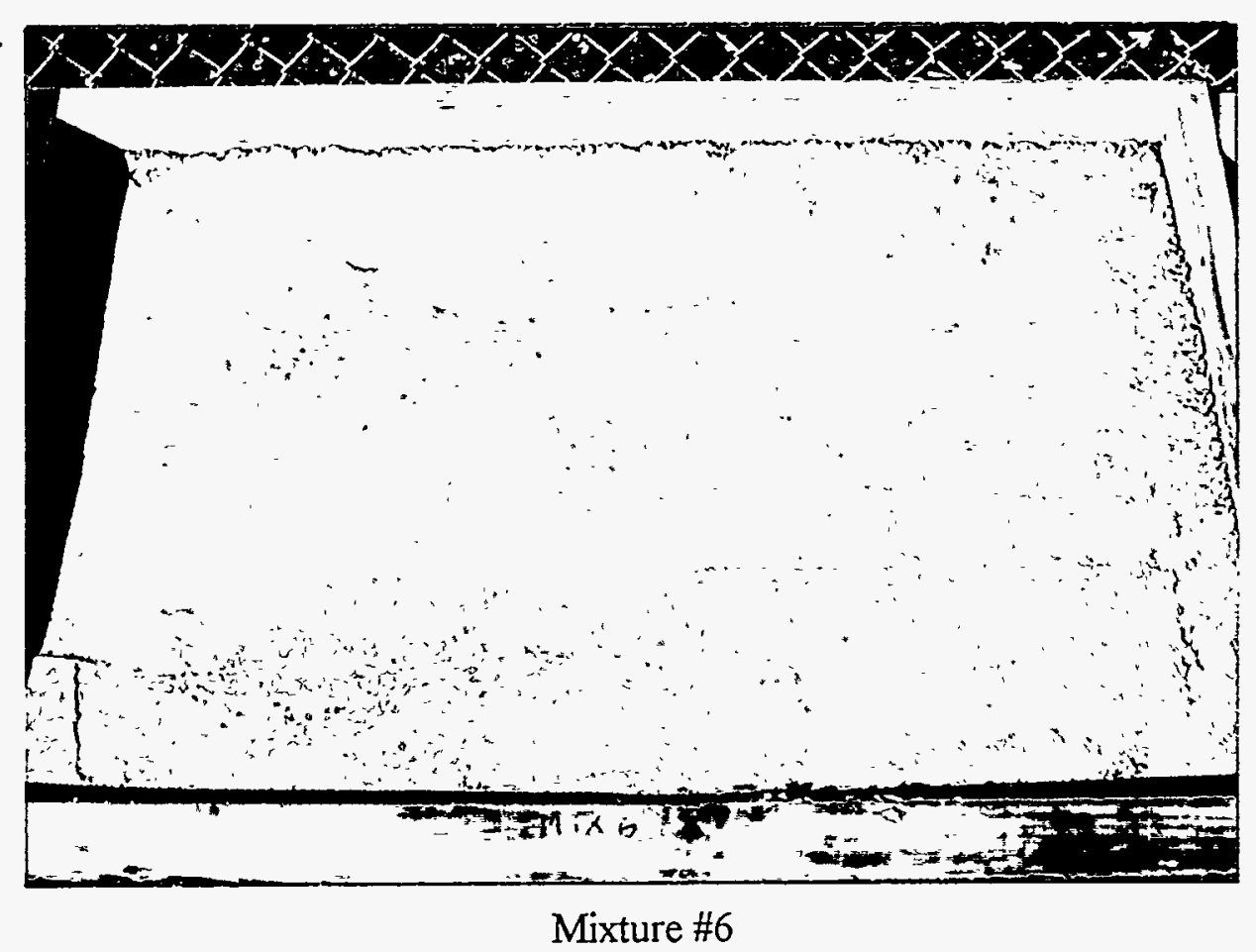



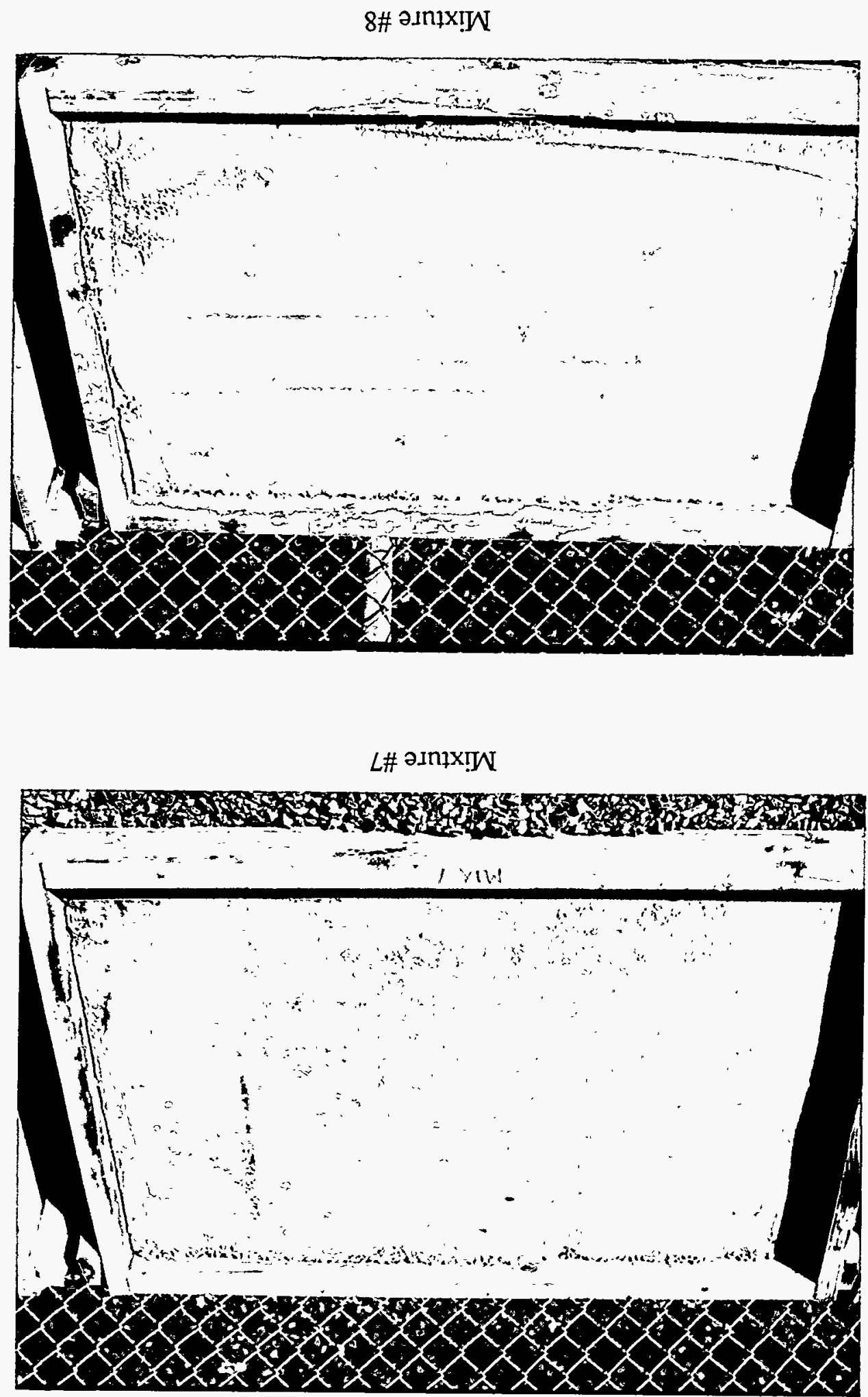


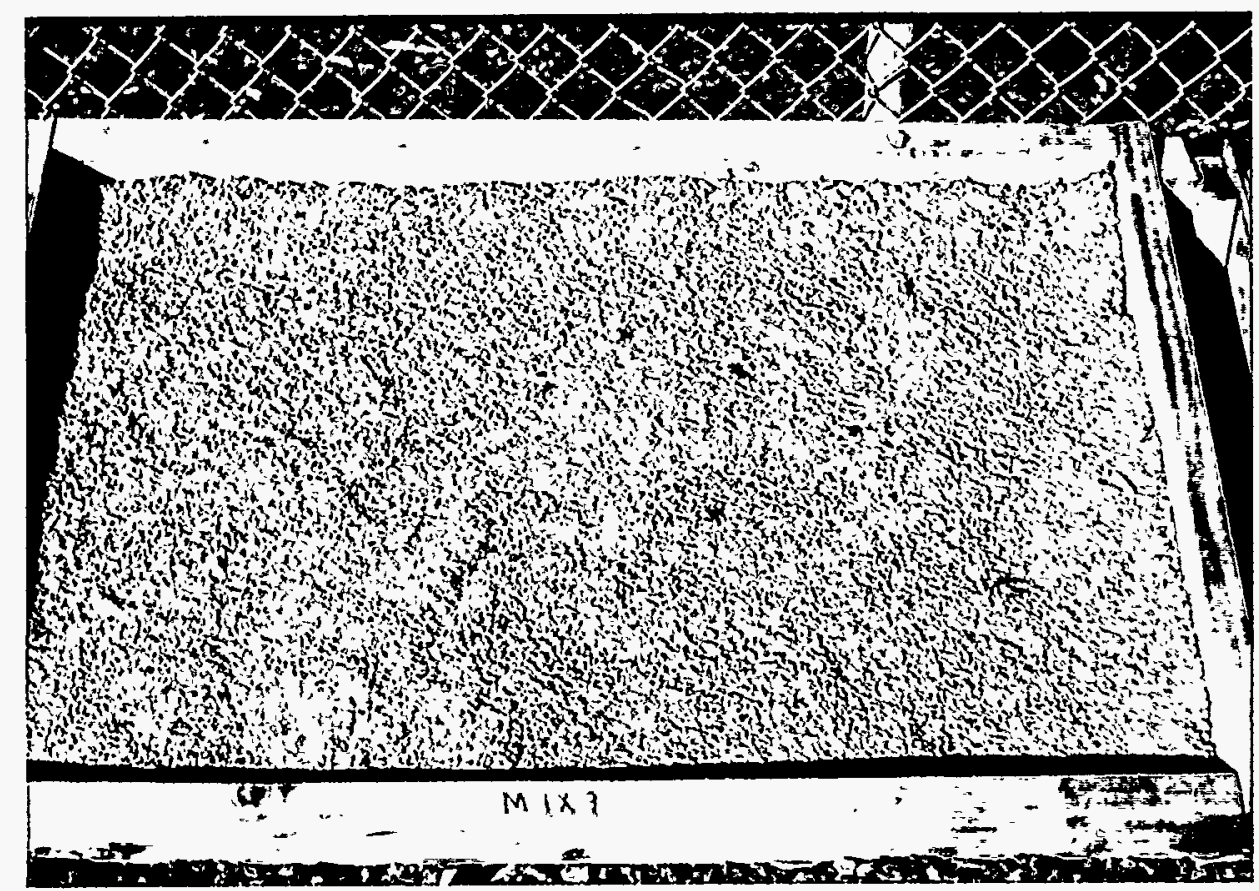

Mixture \#9 

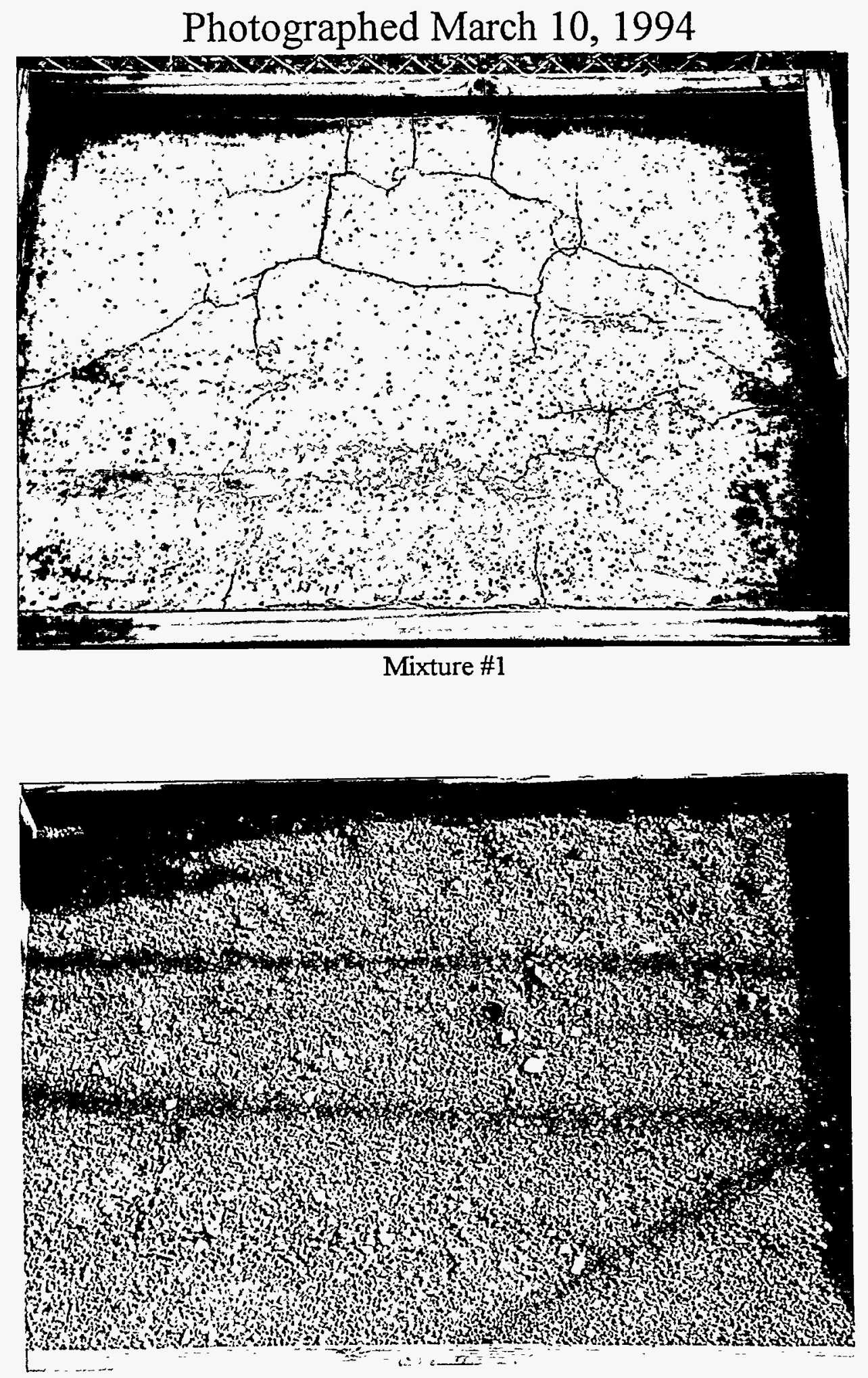

Mixture \#2 

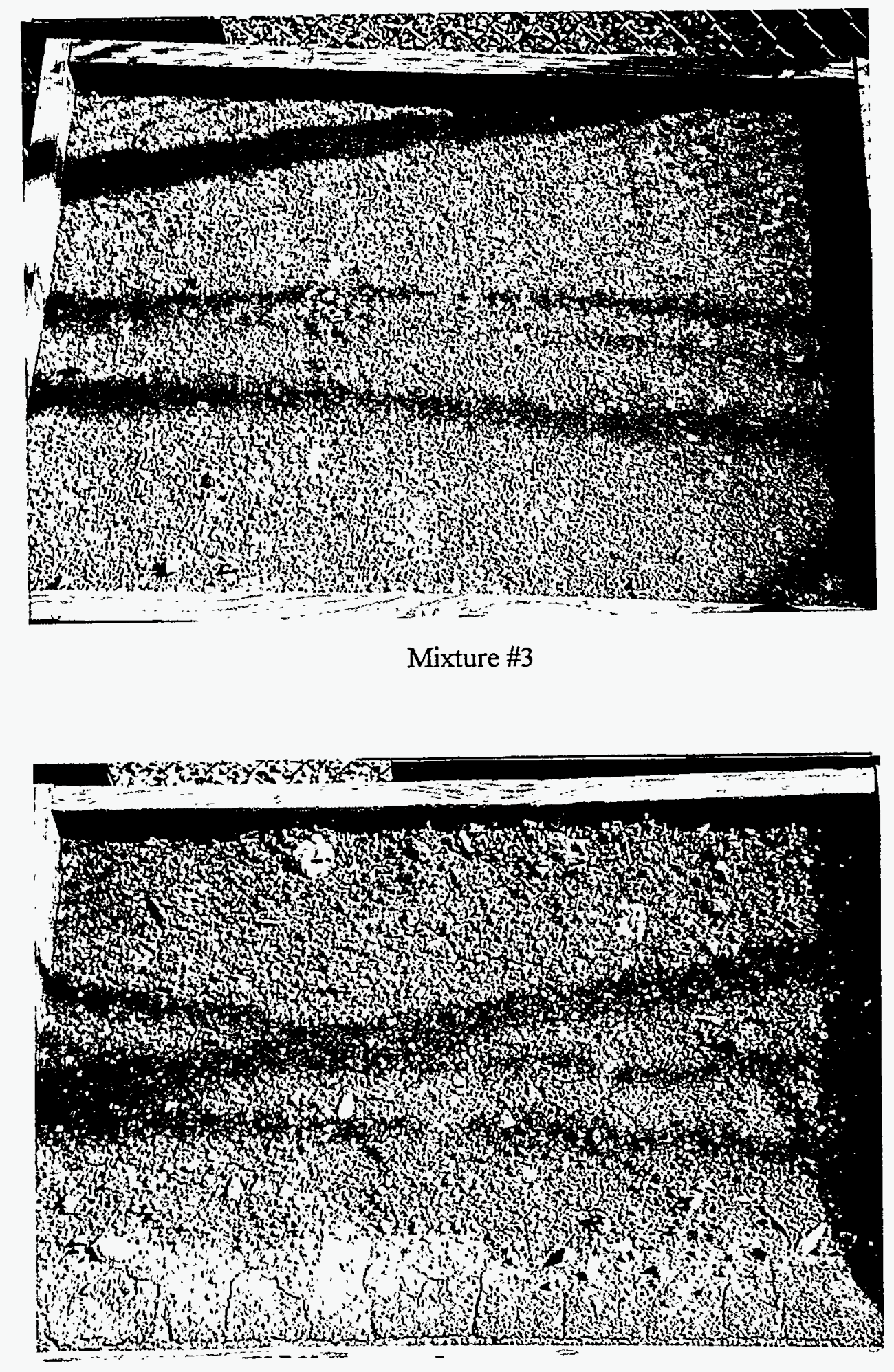

Mixture \#4 

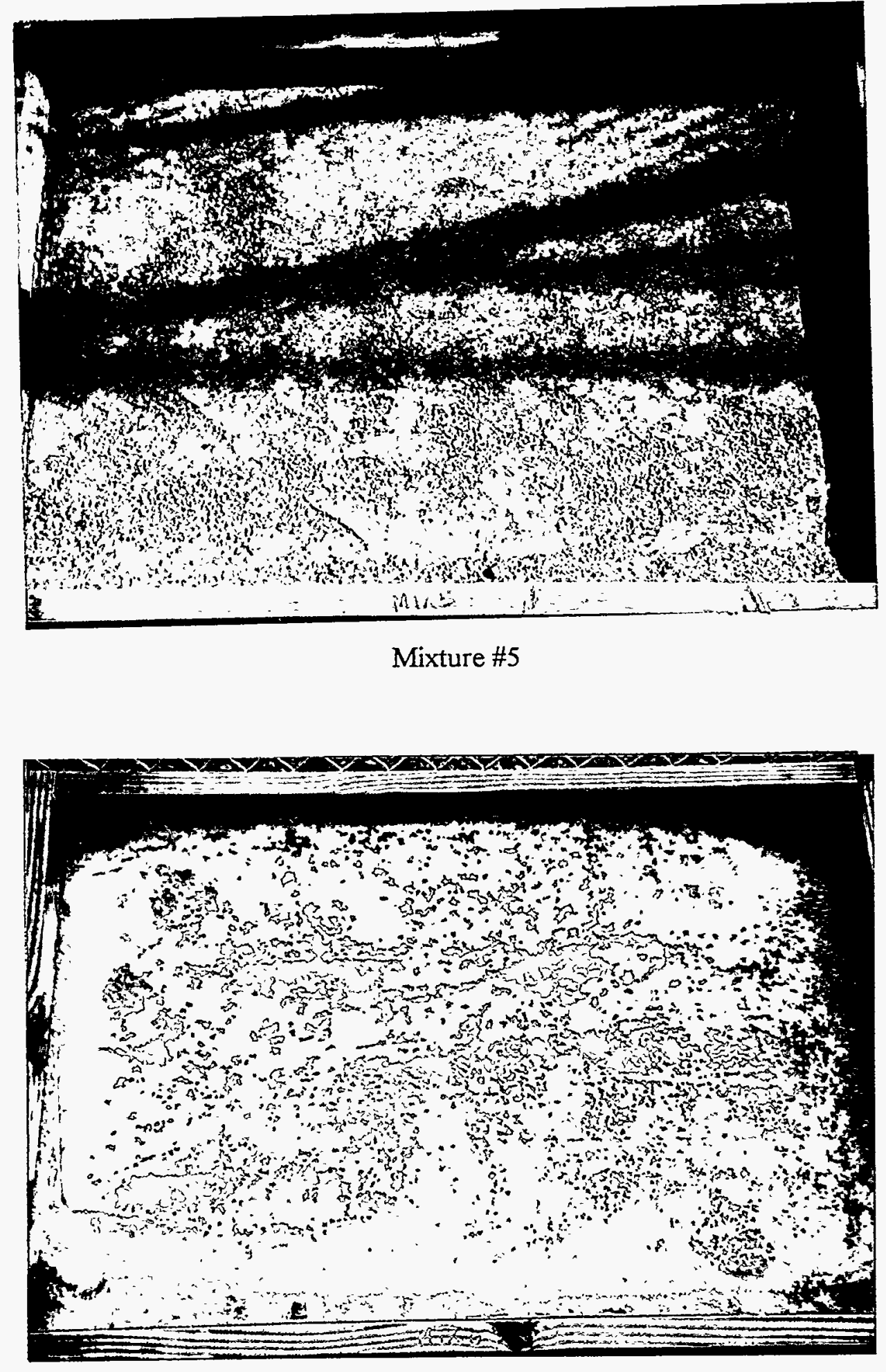

Mixture \#6 


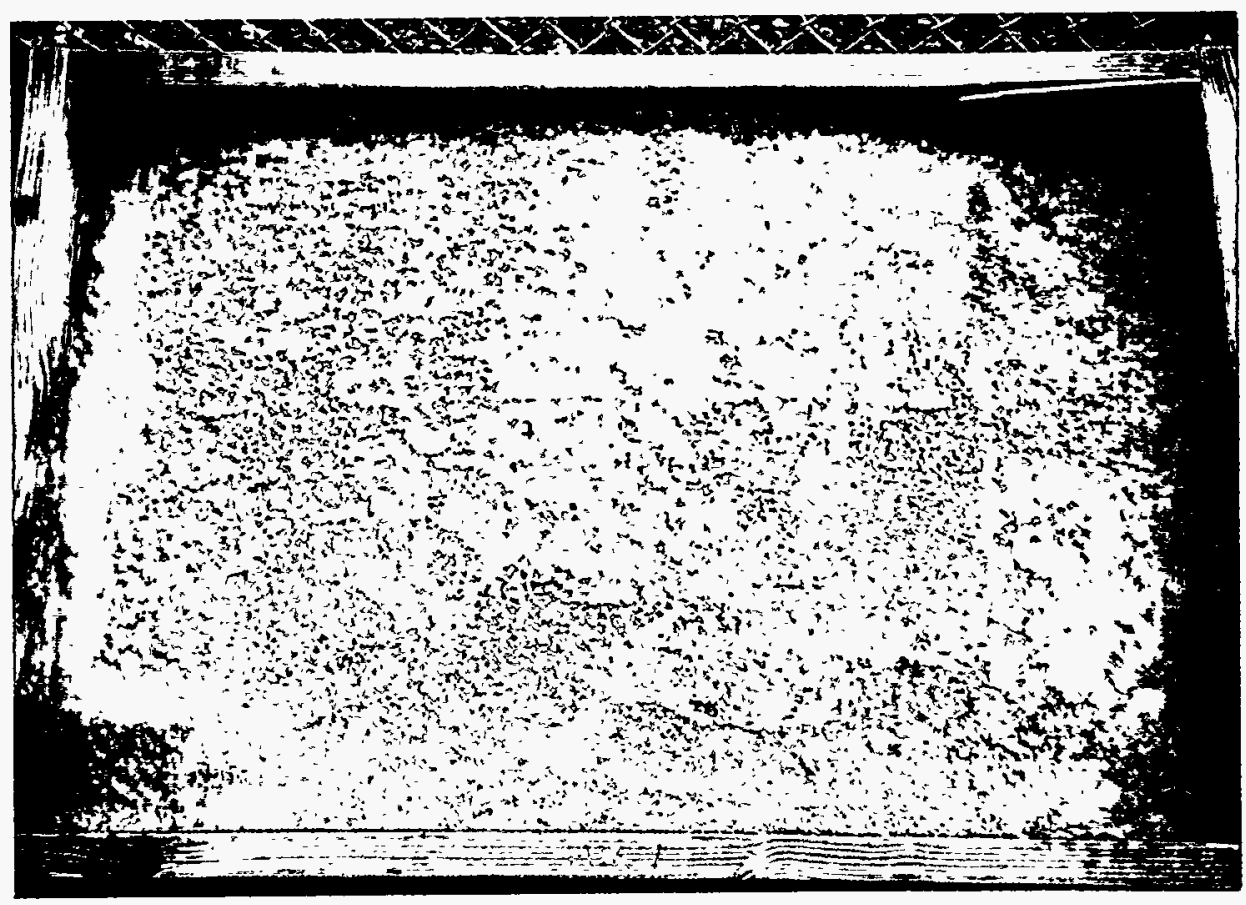

Mixture \#7

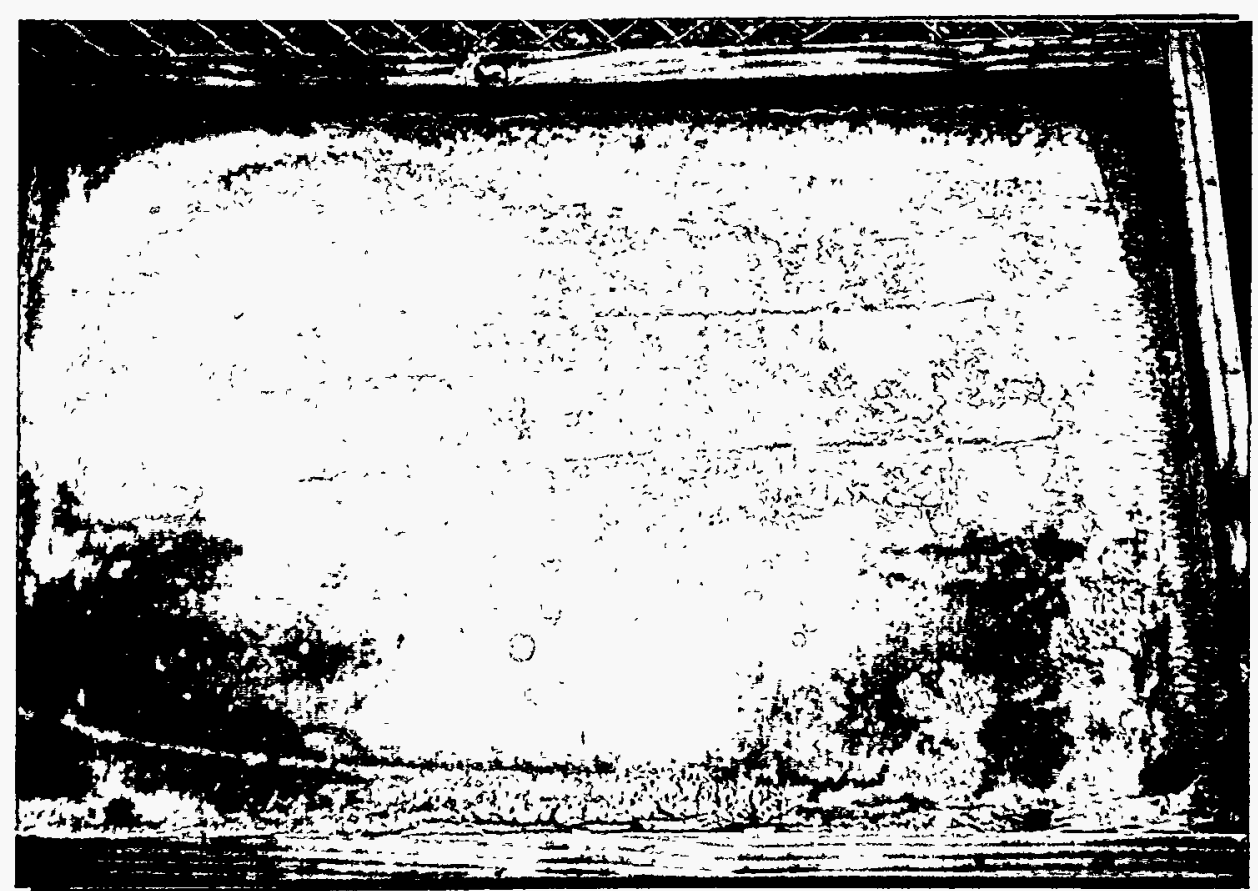

Mixture \#8 


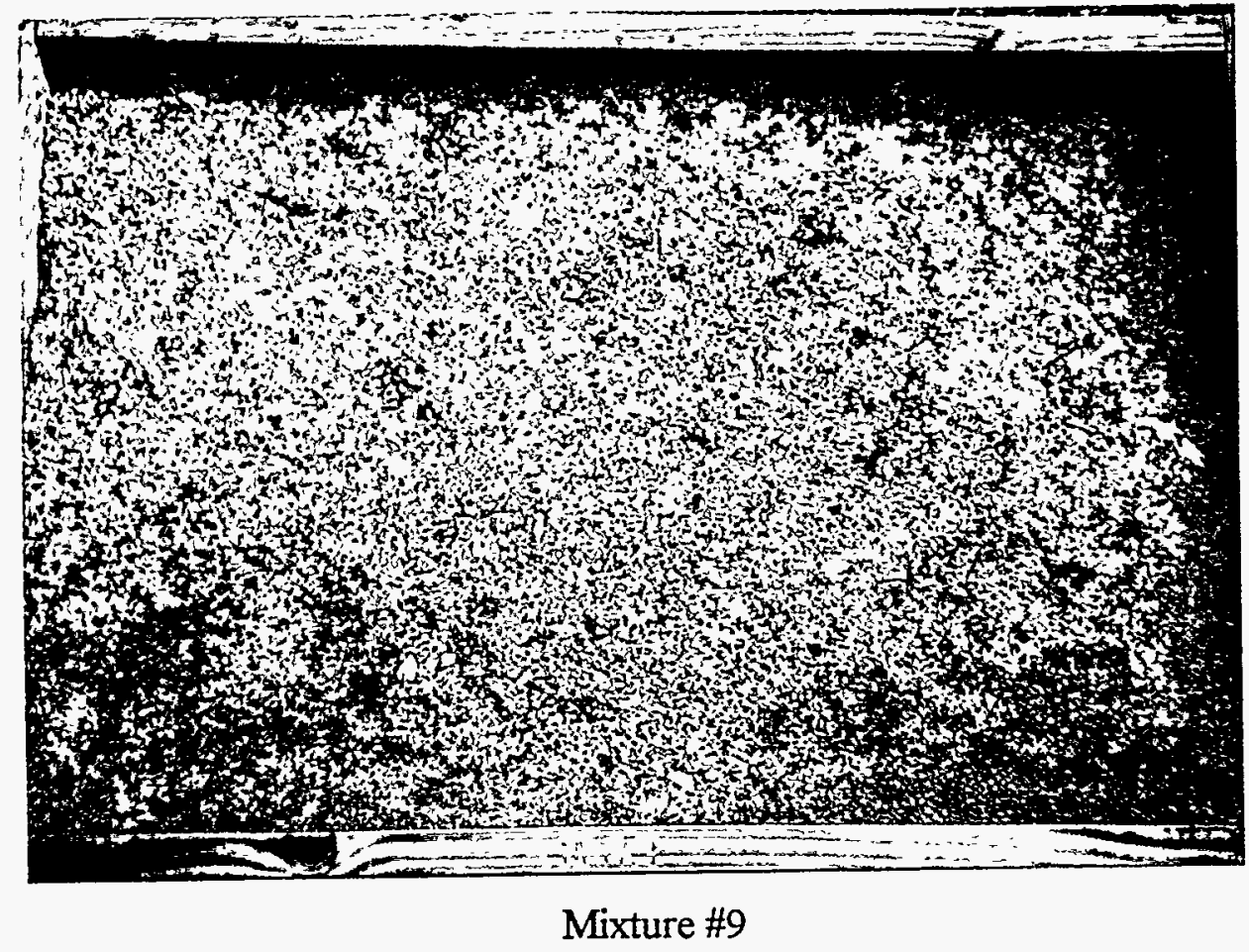




\section{Permeability Test Results}

Three Task 1 ash mixtures (in addition to the control mixture) were chosen for permeability testing: mixtures 1,5 , and 9. These particular mixtures were selected on the assumption that they had the best chance of meeting the required criteria for final cover material.

\section{Procedure}

Prior to running the tests, modified proctor compaction tests were carried out to determine maximum dry density and optimum moisture of each mixture (a compaction test was also run on mixture 3 to determine its moisture-density relationship). The resulting compaction curves are included as Figures 2-6, beginning on page A7-4. A summary of the results appear in Table 1 below.

\begin{tabular}{|c|c|c|}
\hline Mixture & Max Dry Density (pcf) & Optimum Moisture \\
\hline 1 & 81 & 338 \\
\hline 3 & 93 & 238 \\
\hline 4 & 111 & 208 \\
\hline 5 & 74 & 328 \\
\hline 9 & 99 & 228 \\
\hline
\end{tabular}

Table 1

After completing the compaction curves, samples were prepared at 908 of maximum dry density and at a moisture approximately 28 wet of optimum. The falling head permeameter was set up similar to that depicted in Figure 1.

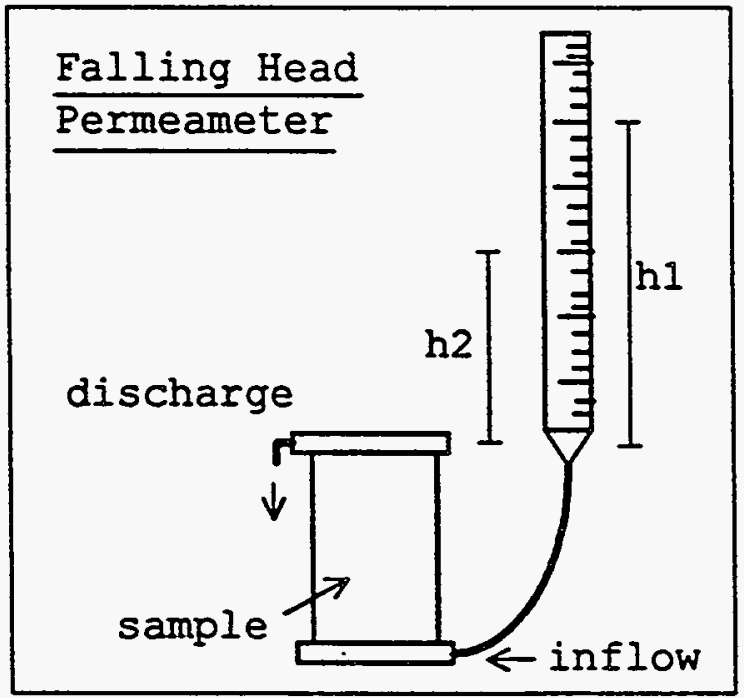


After the sample was prepared, a vacuum was applied to the discharge end of the permeameter to insure complete saturation of the sample. Once the sample was saturated, the vacuum was removed and periodic head measurements were recorded. The coefficient of hydraulic conductivity was then calculated using the following formula:

$$
k=2.303 v l \frac{\log \left(\frac{h_{i}}{h_{f}}\right)}{\left(h_{i}-h_{f}\right) a t}
$$

where

$$
\begin{aligned}
& v=\text { volume change in burette }(\mathrm{ml}) \\
& l=\text { length of sample }(\mathrm{cm}) \\
& h_{i}=\text { initial head difference }(\mathrm{cm}) \\
& h_{f}=\text { final head difference }(\mathrm{cm}) \\
& a=\text { cross sectional area of sample }\left(\mathrm{cm}^{2}\right) \\
& t=\text { duration of test run (sec) }
\end{aligned}
$$

After a sufficient number of runs were carried out (minimum of 4), an average was calculated for that sample. Three individual samples were prepared for each mixture, and an overall average calculated. These results appear in Table 2 below:

\begin{tabular}{|c|c|}
\hline Mixture & $\mathrm{k}(\mathrm{cm} / \mathrm{sec})$ \\
\hline 1 & $1.66 \times 10^{-6}$ \\
\hline 4 & $3.06 \times 10^{-7}$ \\
\hline 5 & $4.39 \times 10^{-6}$ \\
\hline 9 & $1.16 \times 10^{-7}$ \\
\hline
\end{tabular}

Table 2

Original laboratory data can be found in Tables 3-11, beginning on page $\mathrm{A7}-9$.

\section{Conclusions}

The ash mixtures possess very low permeability coefficients, meeting the $1 \times 10^{-5} \mathrm{~cm} / \mathrm{sec}$ minimum criteria required for a final cover infiltration inhibiting liner (see Appendix Al). Pursuant to the results of the erosion resistance testing, permeability tests are also being carried out on mixture 3 to determine whether it can meet the criteria for cover material. Results of this test will be included as an addendum to this appendix. 


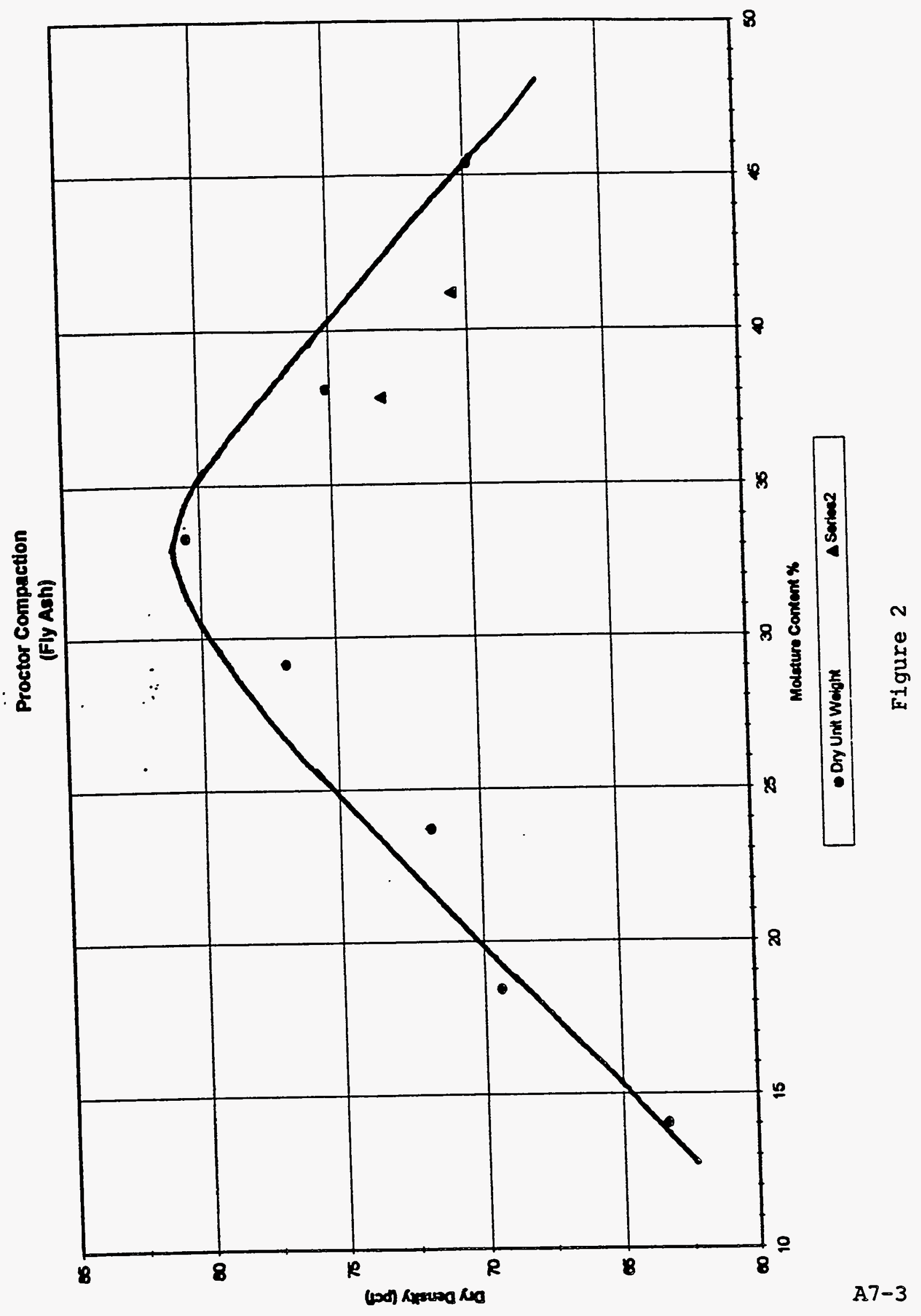




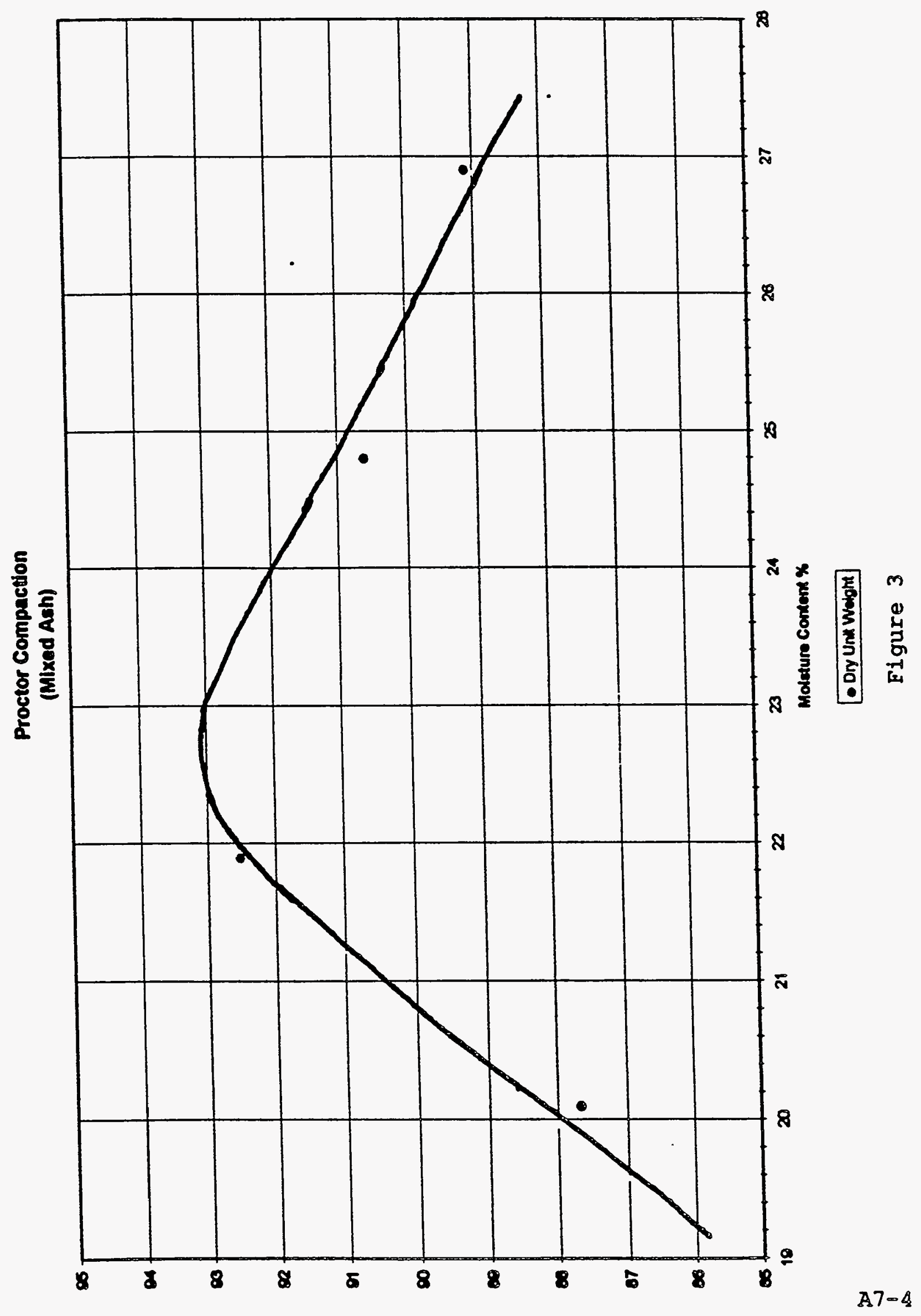




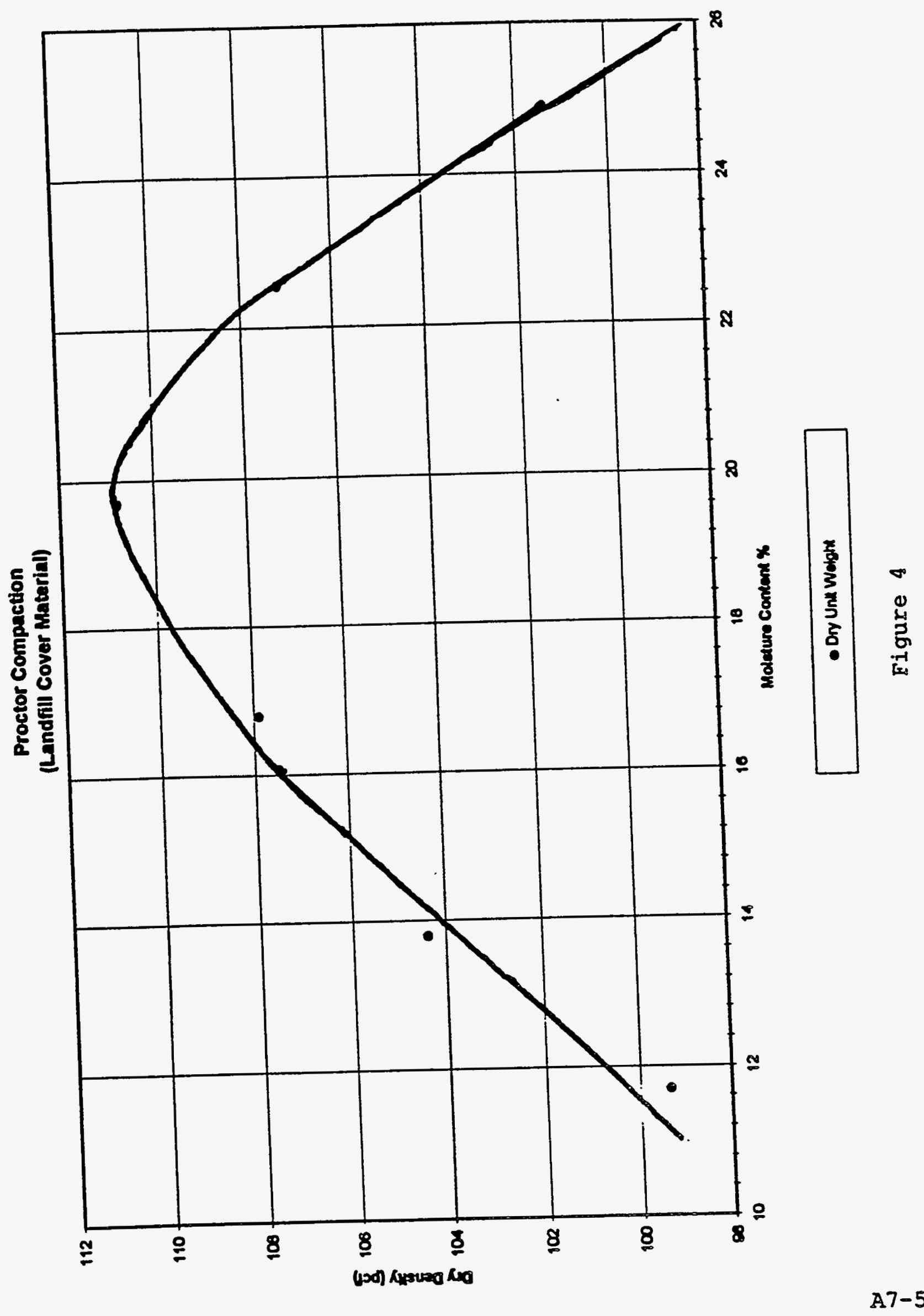




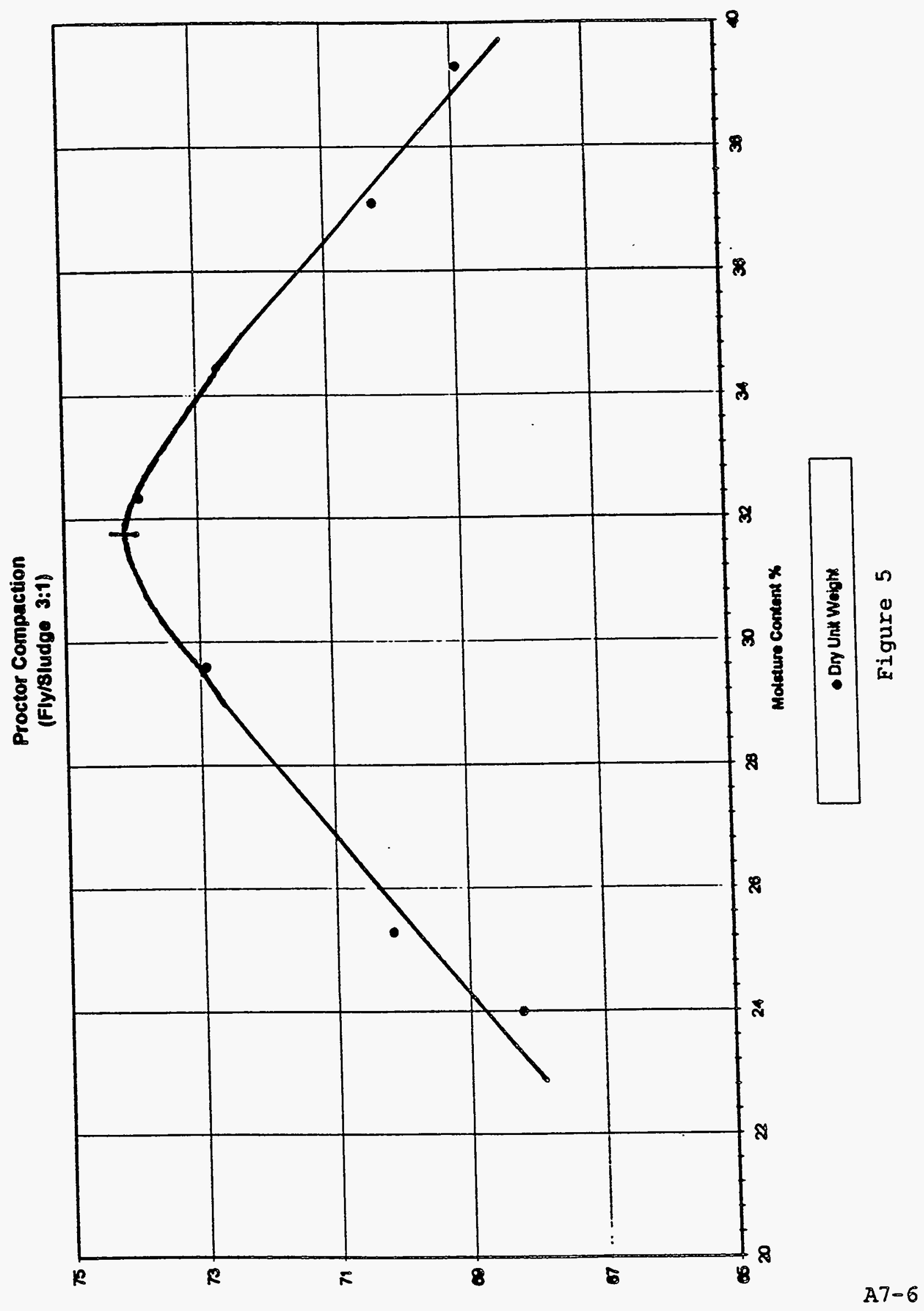


Proctor Compaction

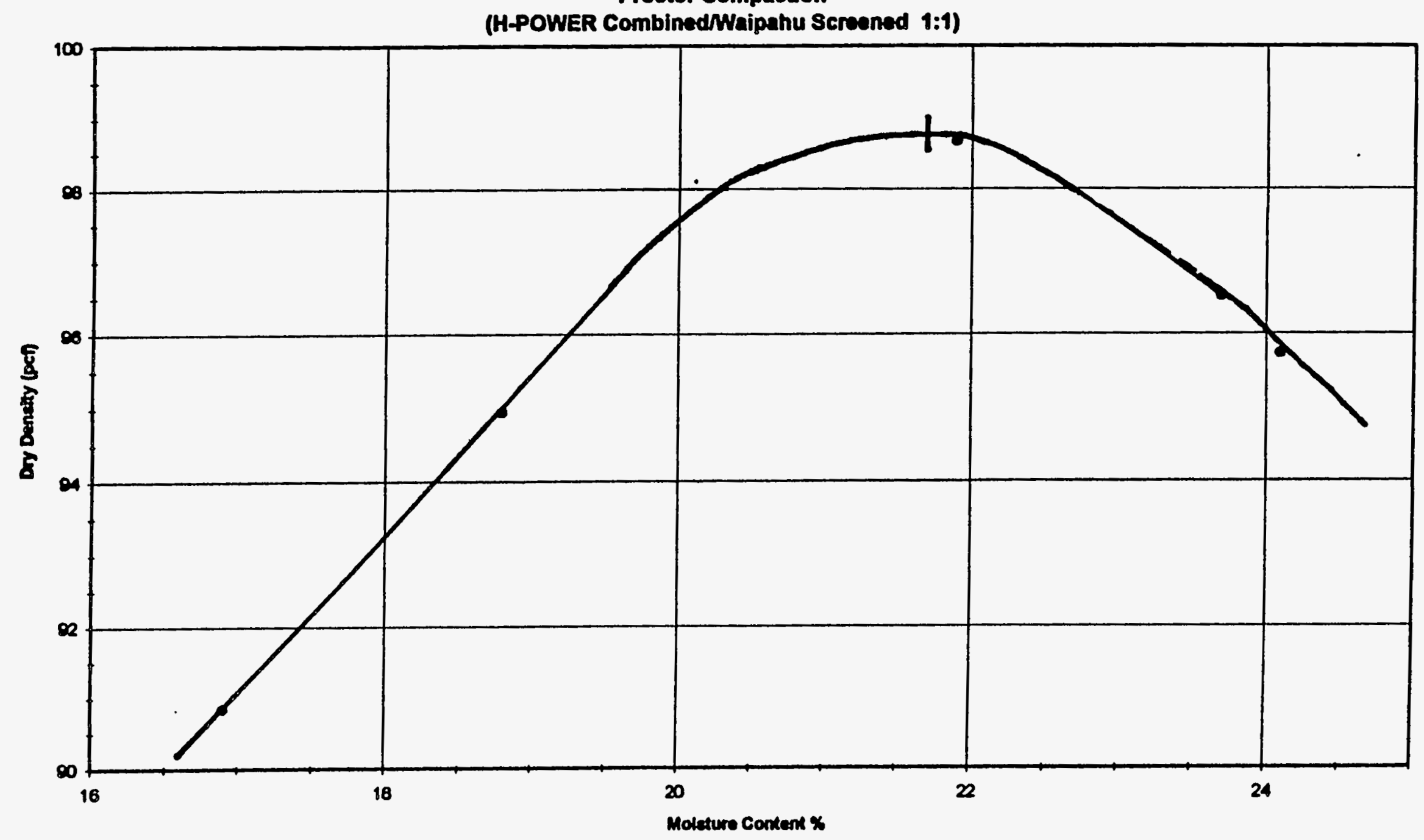

$\stackrel{2}{2}$

- Dry Unit Wolgha

Figure 6 


\begin{tabular}{|r|r|r|r|r|r|r|}
\hline$t(\mathrm{sec})$ & $\mathrm{h}$ init $(\mathrm{cm})$ & $\mathrm{h}$ fin $(\mathrm{cm})$ & vol $(\mathrm{ml})$ & $\mathrm{l}(\mathrm{cm})$ & $\mathrm{a}\left(\mathrm{cm}^{\wedge} 2\right)$ & $\mathrm{k}(\mathrm{cm} / \mathrm{sec})$ \\
\hline & & & & & & \\
\hline 116811 & 74.50 & 71.85 & 4.50 & 14.60 & 32.17 & $2.39 \mathrm{E}-07$ \\
\hline 82524 & 71.85 & 70.25 & 2.70 & 14.60 & 32.17 & $2.09 \mathrm{E}-07$ \\
\hline 142216 & 74.50 & 72.35 & 3.65 & 14.60 & 32.17 & $1.59 \mathrm{E}-07$ \\
\hline 85742 & 72.35 & 71.40 & 1.75 & 14.60 & 32.17 & $1.29 \mathrm{E}-07$ \\
\hline 86087 & 71.40 & 70.45 & 1.40 & 14.60 & 32.17 & $1.04 \mathrm{E}-07$ \\
\hline 303297 & 74.50 & 72.30 & 3.70 & 14.60 & 32.17 & $7.54 \mathrm{E}-08$ \\
\hline 252909 & 72.30 & 72.00 & 0.50 & 14.60 & 32.17 & $1.24 \mathrm{E}-08$ \\
\hline & & & & & Avg & $1.33 \mathrm{E}-07$ \\
\hline
\end{tabular}

Table 3--Mix \#1 (1)

\begin{tabular}{|r|r|r|r|r|r|r|}
\hline$t(\mathrm{sec})$ & $h$ init $(\mathrm{cm})$ & $\mathrm{h}$ fin $(\mathrm{cm})$ & $\mathrm{vol}(\mathrm{ml})$ & $\mathrm{l}(\mathrm{cm})$ & $\mathrm{a}\left(\mathrm{cm}^{\wedge} 2\right)$ & $\mathrm{k}(\mathrm{cm} / \mathrm{sec})$ \\
\hline & & & & & & \\
\hline 660 & 138.1 & 137.14 & 1.6 & 3 & 32.17 & $1.64 \mathrm{E}-06$ \\
\hline 1260 & 137.14 & 135.4 & 2.9 & 3 & 32.17 & $1.58 \mathrm{E}-06$ \\
\hline 600 & 135.4 & 134.608 & 1.32 & 3 & 32.17 & $1.52 \mathrm{E}-06$ \\
\hline 660 & 134.608 & 133.72 & 1.48 & 3 & 32.17 & $1.56 \mathrm{E}-06$ \\
\hline 600 & 133.72 & 132.94 & 1.3 & 3 & 32.17 & $1.52 \mathrm{E}-06$ \\
\hline 600 & 132.94 & 132.19 & 1.25 & 3 & 32.17 & $1.47 \mathrm{E}-06$ \\
\hline 600 & 132.19 & 131.41 & 1.3 & 3 & 32.17 & $1.53 \mathrm{E}-06$ \\
\hline 600 & 131.41 & 130.66 & 1.25 & 3 & 32.17 & $1.48 \mathrm{E}-06$ \\
\hline 240 & 130.66 & 130.3 & 0.6 & 3 & 32.17 & $1.79 \mathrm{E}-06$ \\
\hline 360 & 130.3 & 129.88 & 0.7 & 3 & 32.17 & $1.39 \mathrm{E}-06$ \\
\hline 5940 & 129.88 & 122.62 & 12.1 & 3 & 32.17 & $1.51 \mathrm{E}-06$ \\
\hline 9420 & 122.62 & 112.06 & 17.6 & 3 & 32.17 & $1.49 \mathrm{E}-06$ \\
\hline 1980 & 112.06 & 109.99 & 3.45 & 3 & 32.17 & $1.46 \mathrm{E}-06$ \\
\hline 840 & 109.99 & 109.06 & 1.55 & 3 & 32.17 & $1.57 \mathrm{E}-06$ \\
\hline 2580 & 109.06 & 106.42 & 4.4 & 3 & 32.17 & $1.48 \mathrm{E}-06$ \\
\hline & & & & & Avg & $1.53 \mathrm{E}-06$ \\
\hline
\end{tabular}

Table 4--Mix \#1 (2) 


\begin{tabular}{|r|r|r|r|r|r|r|}
\hline$t(\mathrm{sec})$ & $h$ init $(\mathrm{cm})$ & $\mathrm{h}$ fin $(\mathrm{cm})$ & \multicolumn{1}{l}{ vol $(\mathrm{ml})$} & $l(\mathrm{~cm})$ & $\mathrm{a}\left(\mathrm{cm}^{\wedge} 2\right)$ & $\mathrm{k}(\mathrm{cm} / \mathrm{sec})$ \\
\hline & & & & & & \\
\hline 46800 & 136 & 135.7 & 0.5 & 7.5 & 32.17 & $1.83 \mathrm{E}-08$ \\
\hline 16680 & 135.7 & 135.22 & 0.8 & 7.5 & 32.17 & $8.26 \mathrm{E}-08$ \\
\hline 5400 & 135.22 & 135.1 & 0.2 & 7.5 & 32.17 & $6.39 \mathrm{E}-08$ \\
\hline 6120 & 135.1 & 134.92 & 0.3 & 7.5 & 32.17 & $8.47 \mathrm{E}-08$ \\
\hline 45300 & 134.92 & 133.72 & 2 & 7.5 & 32.17 & $7.66 \mathrm{E}-08$ \\
\hline 13800 & 133.72 & 133.36 & 0.6 & 7.5 & 32.17 & $7.59 \mathrm{E}-08$ \\
\hline 17400 & 133.36 & 132.94 & 0.7 & 7.5 & 32.17 & $7.05 \mathrm{E}-08$ \\
\hline 6300 & 132.94 & 132.76 & 0.3 & 7.5 & 32.17 & $8.36 \mathrm{E}-08$ \\
\hline 46800 & 132.76 & 131.56 & 2 & 7.5 & 32.17 & $7.54 \mathrm{E}-08$ \\
\hline 6300 & 131.56 & 131.43 & 0.22 & 7.5 & 32.17 & $6.19 \mathrm{E}-08$ \\
\hline 3600 & 131.43 & 131.32 & 0.18 & 7.5 & 32.17 & $8.87 \mathrm{E}-08$ \\
\hline 19800 & 131.32 & 130.84 & 0.8 & 7.5 & 32.17 & $7.19 \mathrm{E}-08$ \\
\hline 7200 & 130.84 & 130.66 & 0.3 & 7.5 & 32.17 & $7.43 \mathrm{E}-08$ \\
\hline 221700 & 130.66 & 124.6 & 10.1 & 7.5 & 32.17 & $8.32 \mathrm{E}-08$ \\
\hline 39600 & 124.6 & 124.3 & 0.5 & 7.5 & 32.17 & $2.37 \mathrm{E}-08$ \\
\hline & & & & & Avg & $6.90 \mathrm{E}-08$ \\
\hline
\end{tabular}

Table 5--Mix \#4 (1) 


\begin{tabular}{|c|c|c|c|c|c|c|}
\hline$t(\sec )$ & $h$ init $(\mathrm{cm})$ & $\mathrm{h}$ fin $(\mathrm{cm})$ & vol (ml) & I (cm) & $a\left(\mathrm{~cm}^{\wedge} 2\right)$ & $k(\mathrm{~cm} / \mathrm{sec})$ \\
\hline 9600 & 138.1 & 135.46 & 44 & 75 & 3217 & $781 \mathrm{~F}-07$ \\
\hline 6000 & 135.46 & 134.02 & 2.4 & 7.5 & 32.17 & $6.92 E-07$ \\
\hline 1800 & 134.02 & 133.54 & 0.8 & 7.5 & 32.17 & $7.75 \mathrm{E}-07$ \\
\hline 5400 & 133.54 & 132.28 & 2.1 & 7.5 & 32.17 & $6.82 \mathrm{E}-07$ \\
\hline 1500 & 132.28 & 131.98 & 0.5 & 7.5 & 32.17 & 5.88E-07 \\
\hline 5100 & 131.98 & 130.9 & 1.8 & 7.5 & 32.17 & $6.26 \mathrm{E}-07$ \\
\hline 4200 & 130.9 & 130.03 & 1.45 & 7.5 & 32.17 & $6.17 E-07$ \\
\hline 4200 & 130.03 & 129.16 & 1.45 & 7.5 & 32.17 & $6.21 \mathrm{E}-07$ \\
\hline 37800 & 129.16 & 122.14 & 11.7 & 7.5 & 32.17 & $5.75 E-07$ \\
\hline 9780 & 122.14 & 120.58 & 2.6 & 7.5 & 32.17 & 5.11E-07 \\
\hline 10440 & 120.58 & 118.9 & 2.8 & 7.5 & 32.17 & $5.22 E-07$ \\
\hline 3180 & 118.9 & 118.42 & 0.8 & 7.5 & 32.17 & $4.94 \mathrm{E}-07$ \\
\hline 4800 & 118.42 & 117.7 & 1.2 & 7.5 & 32.17 & $4.94 E-07$ \\
\hline 4800 & 117.7 & 116.98 & 1.2 & 7.5 & 32.17 & $4.97 E-07$ \\
\hline 6300 & 116.98 & 116.02 & 1.6 & 7.5 & 32.17 & $5.08 \mathrm{E}-07$ \\
\hline 3600 & 116.02 & 115.48 & 0.9 & 7.5 & 33.17 & $4.88 E-07$ \\
\hline 47100 & 115.48 & 109.06 & 10.7 & 7.5 & 34.17 & 4.44E-07 \\
\hline 9180 & 109.06 & 107.98 & 1.8 & 7.5 & 35.17 & $3.85 E-07$ \\
\hline 2820 & 107.98 & 107.38 & 1 & 7.5 & 36.17 & $6.83 E-07$ \\
\hline 8400 & 107.38 & 106.42 & 1.6 & 7.5 & 37.17 & $3.60 E-07$ \\
\hline 8400 & 106.42 & 105.4 & 1.7 & 7.5 & 38.17 & $3.76 E-07$ \\
\hline 13800 & 105.4 & 103.54 & 3.1 & 7.5 & 39.17 & $4.12 E-07$ \\
\hline 47400 & 103.54 & 98.14 & 9 & 7.5 & \begin{tabular}{|l|}
40.17 \\
\end{tabular} & $3.52 \mathrm{E}-07$ \\
\hline & & & & \multicolumn{2}{|c|}{ Avg } & $5.43 E-07$ \\
\hline
\end{tabular}

Table 6--Mix \#4 (2) 


\begin{tabular}{|r|r|r|r|r|r|r|}
\hline $\mathrm{t}(\mathrm{sec})$ & $\mathrm{h}$ init $(\mathrm{cm})$ & $\mathrm{h}$ fin $(\mathrm{cm})$ & $\mathrm{vol}(\mathrm{ml})$ & $\mathrm{l}(\mathrm{cm})$ & $\mathrm{a}\left(\mathrm{cm}^{\wedge} 2\right)$ & $\mathrm{k}(\mathrm{cm} / \mathrm{sec})$ \\
\hline & & & & & & \\
\hline 1326 & 70.4 & 68 & 4 & 7.5 & 32.17 & $1.02 \mathrm{E}-05$ \\
\hline 1313 & 67.4 & 65 & 4 & 7.5 & 32.17 & $1.07 \mathrm{E}-05$ \\
\hline 1437 & 63.8 & 61.4 & 4 & 7.5 & 32.17 & $1.04 \mathrm{E}-05$ \\
\hline 1162 & 78 & 75.6 & 4 & 7.5 & 32.17 & $1.05 \mathrm{E}-05$ \\
\hline 1250 & 74.4 & 72.05 & 4 & 7.5 & 32.17 & $1.02 \mathrm{E}-05$ \\
\hline 2454 & 78 & 73.25 & 8 & 7.5 & 32.17 & $1.01 \mathrm{E}-05$ \\
\hline 2661 & 72.9 & 68.2 & 8 & 7.5 & 32.17 & $9.94 \mathrm{E}-06$ \\
\hline & & & & & \multicolumn{1}{l}{ Avg } & $1.03 \mathrm{E}-05$ \\
\hline
\end{tabular}

Table 7--Mix \#5 (1)

\begin{tabular}{|r|r|r|r|r|r|r|}
\hline$t(\mathrm{sec})$ & $\mathrm{h}$ init $(\mathrm{cm})$ & $\mathrm{h}$ fin $(\mathrm{cm})$ & vol $(\mathrm{ml})$ & $\mathrm{l}(\mathrm{cm})$ & $\mathrm{a}\left(\mathrm{cm}^{\wedge} 2\right)$ & $\mathrm{k}(\mathrm{cm} / \mathrm{sec})$ \\
\hline & & & & & & \\
\hline 116811 & 72.7 & 57.1 & 26.4 & 7.5 & 32.17 & $8.16 \mathrm{E}-07$ \\
\hline 82524 & 57.1 & 48.9 & 13.8 & 7.5 & 32.17 & $7.37 \mathrm{E}-07$ \\
\hline 142216 & 72.7 & 63.1 & 16.3 & 7.5 & 32.17 & $3.94 \mathrm{E}-07$ \\
\hline 85742 & 63.1 & 61.25 & 1.2 & 7.5 & 32.17 & $5.25 \mathrm{E}-08$ \\
\hline & & & & \multicolumn{2}{l}{ Avg } & $5.00 \mathrm{E}-07$ \\
\hline
\end{tabular}

Table 8--Mix \#5 (2)

\begin{tabular}{|r|r|r|r|r|r|r|}
\hline$t(\mathrm{sec})$ & h init $(\mathrm{cm})$ & $\mathrm{h}$ fin $(\mathrm{cm})$ & $\mathrm{vol}(\mathrm{ml})$ & $\mathrm{l}(\mathrm{cm})$ & $\mathrm{a}\left(\mathrm{cm}^{\wedge} 2\right)$ & $k(\mathrm{~cm} / \mathrm{sec})$ \\
\hline & & & & & & \\
\hline 92718 & 83.6 & 64.25 & 32.8 & 7.5 & 32.17 & $1.12 \mathrm{E}-06$ \\
\hline 126506 & 83.6 & 59.5 & 40.6 & 7.5 & 32.17 & $1.06 \mathrm{E}-06$ \\
\hline 2881 & 218.9 & 213.1 & 8.6 & 7.5 & 32.17 & $3.22 \mathrm{E}-06$ \\
\hline 8217 & 213.1 & 200.1 & 22.7 & 7.5 & 32.17 & $3.12 \mathrm{E}-06$ \\
\hline 37996 & 218.9 & 166.8 & 86.4 & 7.5 & 32.17 & $2.77 \mathrm{E}-06$ \\
\hline 21177 & 218.9 & 191 & 45.1 & 7.5 & 32.17 & $2.43 \mathrm{E}-06$ \\
\hline 26571 & 218.9 & 186.5 & 55 & 7.5 & 32.17 & $2.39 \mathrm{E}-06$ \\
\hline 42670 & 218.9 & 173.1 & 77.6 & 7.5 & 32.17 & $2.17 \mathrm{E}-06$ \\
\hline 23994 & 218.9 & 193.3 & 43.4 & 7.5 & 32.17 & $2.05 \mathrm{E}-06$ \\
\hline & & & & & Avg & $2.26 \mathrm{E}-06$ \\
\hline
\end{tabular}

Table 9--Mix \#5 (3) 


\begin{tabular}{|r|r|r|r|r|r|r|}
\hline$t$ (sec) & $h$ init $(\mathrm{cm})$ & $h$ fin $(\mathrm{cm})$ & vol $(\mathrm{ml})$ & $l(\mathrm{~cm})$ & $\mathrm{a}\left(\mathrm{cm}^{\wedge} 2\right)$ & $k(\mathrm{~cm} / \mathrm{sec})$ \\
\hline & & & & & & \\
\hline 116811 & 79.75 & 77 & 4.6 & 7.5 & 32.17 & $1.17 \mathrm{E}-07$ \\
\hline 82524 & 77 & 75.15 & 3.2 & 7.5 & 32.17 & $1.19 \mathrm{E}-07$ \\
\hline 142216 & 79.75 & 76.35 & 5.7 & 7.5 & 32.17 & $1.20 \mathrm{E}-07$ \\
\hline 85742 & 76.35 & 74.4 & 3.3 & 7.5 & 32.17 & $1.19 \mathrm{E}-07$ \\
\hline 86087 & 74.4 & 72.5 & 3.2 & 7.5 & 32.17 & $1.18 \mathrm{E}-07$ \\
\hline 303297 & 79.75 & 72.65 & 12 & 7.5 & 32.17 & $1.21 \mathrm{E}-07$ \\
\hline 252909 & 72.65 & 68.1 & 7.7 & 7.5 & 32.17 & $1.01 \mathrm{E}-07$ \\
\hline & & & & & \multicolumn{3}{l}{ Avg } & $1.16 \mathrm{E}-07$ \\
\hline
\end{tabular}

Table 10--Mix \#9 (1)

\begin{tabular}{|r|r|r|r|r|r|r|}
\hline$t(\mathrm{sec})$ & $\mathrm{h}$ init $(\mathrm{cm})$ & $\mathrm{h}$ fin $(\mathrm{cm})$ & $\mathrm{vol}(\mathrm{ml})$ & $\mathrm{l}(\mathrm{cm})$ & $\mathrm{a}\left(\mathrm{cm}^{\wedge} 2\right)$ & $\mathrm{k}(\mathrm{cm} / \mathrm{sec})$ \\
\hline & & & & & & \\
\hline 17400 & 103.7 & 102.74 & 1.6 & 7.5 & 32.17 & $2.08 \mathrm{E}-07$ \\
\hline 6300 & 102.74 & 102.44 & 0.5 & 7.5 & 32.17 & $1.80 \mathrm{E}-07$ \\
\hline 46800 & 102.44 & 100.34 & 3.5 & 7.5 & 32.17 & $1.72 \mathrm{E}-07$ \\
\hline 6300 & 100.34 & 100.1 & 0.4 & 7.5 & 32.17 & $1.48 \mathrm{E}-07$ \\
\hline 3600 & 100.1 & 99.92 & 0.3 & 7.5 & 32.17 & $1.94 \mathrm{E}-07$ \\
\hline 19800 & 99.92 & 99.14 & 1.3 & 7.5 & 32.17 & $1.54 \mathrm{E}-07$ \\
\hline 7200 & 99.14 & 98.9 & 0.4 & 7.5 & 32.17 & $1.31 \mathrm{E}-07$ \\
\hline 49500 & 98.9 & 93.38 & 9.2 & 7.5 & 32.17 & $4.50 \mathrm{E}-07$ \\
\hline 39600 & 93.38 & 93.2 & 0.3 & 7.5 & 32.17 & $1.84 \mathrm{E}-08$ \\
\hline & & & & & Avg & $1.84 \mathrm{E}-07$ \\
\hline
\end{tabular}

Table 11--Mix \#9 (2) 


\section{Ash TCLP Test Results}

H-POWER has been conducting leachate testing on its ash since the plant became operational in 1989. To date, over 400 samples have consistently tested well below EPA limits for toxic metal concentrations. Table 1 below summarizes the results of the leachate testing. The figures on pages 2 and 3 provide a graphical representation of this data.

\begin{tabular}{|c|c|c|c|c|c|c|c|c|c|c|c|c|}
\hline & & Arsenic & Barium & Cadmium & Chromium & Lead & Mercury & Selenium & Silver & Copper & TCDD & TCDF \\
\hline & $\begin{array}{l}\text { EPA STD } \\
\text { MGR }\end{array}$ & 5 & 100 & 1 & 5 & 5 & 0.2 & 1 & 5 & - & ng/gm & $\mathrm{ng} / \mathrm{gm}$ \\
\hline & $\begin{array}{l}\text { EPA Drinking } \\
\text { Water MG }\end{array}$ & 0.05 & 1 & 0.01 & 0.05 & 0.05 & 0.002 & 0.01 & 0.05 & - & $\begin{array}{l}\text { [no limits now } \\
\text { available] }\end{array}$ & \\
\hline Dec-89 & & 1.64 & 0.4 & 0.34 & 0.005 & 1.88 & 0.003 & 0.64 & 0.03 & & & \\
\hline Fob-90 & & 1.23 & 0.21 & 0.09 & 0.05 & 0.64 & 0.00002 & 0.42 & 0.03 & & & \\
\hline Jun-90 & & 1.42 & 0.08 & 0.16 & 0.03 & 0.4 & 0.008 & 0.44 & 0.03 & & & \\
\hline Jut90 & & 1.72 & 0.19 & 0.16 & 0.02 & 1.22 & 0.00056 & 0.28 & 0.02 & & & \\
\hline Aug.90 & & 1.47 & 1.82 & 0.23 & 0.22 & 1.49 & 0.001 & 0.72 & 0.02 & & & \\
\hline Sop-90 & & 0.77 & 0.41 & 0.21 & 0.04 & 0.92 & 0.002 & 0.35 & 0.05 & & & \\
\hline oct.90 & 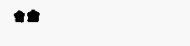 & 0.407 & 1.53 & 0.017 & $0 . \infty 2$ & 0.23 & 0.003 & 0.1 & 0.02 & & & \\
\hline Nov.90 & & 0.28 & 0.18 & 0.28 & 0.04 & 1.08 & 0.02 & 0.08 & 0.05 & & & \\
\hline Dec.90 & & 0.1 & 0.07 & 0.29 & 0.04 & 2.73 & 0.0008 & 0.04 & 0.06 & & & \\
\hline Jan-91 & & 0.1 & 0.00 & 0.21 & 0.03 & 0.54 & 0.004 & 0.04 & 0.03 & & & \\
\hline Feb-91 & $* *$ & 0.2 & 1.45 & 0.02 & 0.02 & 0.52 & 0.0008 & 0.08 & 0.05 & & & \\
\hline Mar-91 & & 0.07 & 0.18 & 0.18 & 0.03 & 0.25 & 0.01 & 0.05 & 0.05 & 0.09 & & \\
\hline Apr-91 & & 0.065 & 0.042 & 0.118 & 0.032 & 0.0635 & 0.00095 & 0.047 & 0.04 & & & \\
\hline May-91 & & 0.042 & 0.074 & 0.102 & 0.029 & 0.152 & 0.0033 & 0.033 & 0.018 & & & \\
\hline Jun-91 & & 0.5 & 8.05 & 0.142 & 0.5 & 0.74 & 0.00068 & 0.1 & 0.5 & & & \\
\hline Jut-91 & & 0.133 & 0.045 & 0.117 & 0.033 & 0.102 & 0.002 & 0.034 & 0.044 & & & \\
\hline Aug-91 & $\because$ & 0.12 & 0.88 & 0.02 & 0.02 & 0.33 & 0.005 & 0.08 & 0.19 & & & \\
\hline Sop-91 & & 0.15 & 0.13 & 0.12 & 0.03 & 0.16 & 0.0003 & 0.27 & 0.03 & & & \\
\hline Dec-91 & & 0.1 & 0.07 & 0.24 & 0.028 & 0.8 & 0.0016 & 0.047 & 0.018 & & & \\
\hline Mar.92 & & 0.08 & 0.1 & 0.08 & 0.01 & 0.08 & 0.00099 & 0.04 & 0.02 & & 0.267 & 1.37 \\
\hline May-92 & & 0.1 & 0.25 & 0.19 & 0.02 & 0.181 & 0.00016 & 0.1 & 0.01 & & 0.305 & 1.75 \\
\hline Aug-92 & & 0.09 & 0.12 & 0.3 & 0.028 & 2.08 & 0.0007 & 0.08 & 0.04 & & 0.143 & 1 \\
\hline Dec-92 & & 0.09 & 0.106 & 0.47 & 0.045 & 1.73 & 0.0034 & 0.0662 & 0.0392 & & & \\
\hline Mar.93 & & 0.05 & 0.89 & 0.23 . & 0.01 & 0.34 & 0.00505 & 0.05 & 0.04 & & & \\
\hline Jun-93 & & 0.05 & 0.89 & 0.21 & 0.01 & 0.58 & 0.0038 & 0.05 & 0.05 & & & \\
\hline Sep-93 & & 0.05 & 1.14 & 0.01 & 0.01 & 0.26 & 0.00134 & 0.05 & 0.05 & & 1.272 & 9.035 \\
\hline Doc.93 & & 0.05 & 0.89 & 0.05 & 0.01 & 0.25 & 0.0015 & 0.05 & 0.05 & & 0.427 & 2.85 \\
\hline Mar-94 & & 0.05 & 0.61 & 0.08 & 0.01 & 0.108 & 0.0012 & 0.05 & 0.01 & & & \\
\hline ERAGE & & 0.397393 & 0.745607 & 0.166643 & 0.0488571 & 0.709161 & 0.003063 & 0.155971 & 0.056686 & 0.09 & & \\
\hline
\end{tabular}

-1" Trimester testing using water leachate per EPA Method SW924

Maximum values of three batches tested as reported in ABB it dated 1/31/91

Table 1 

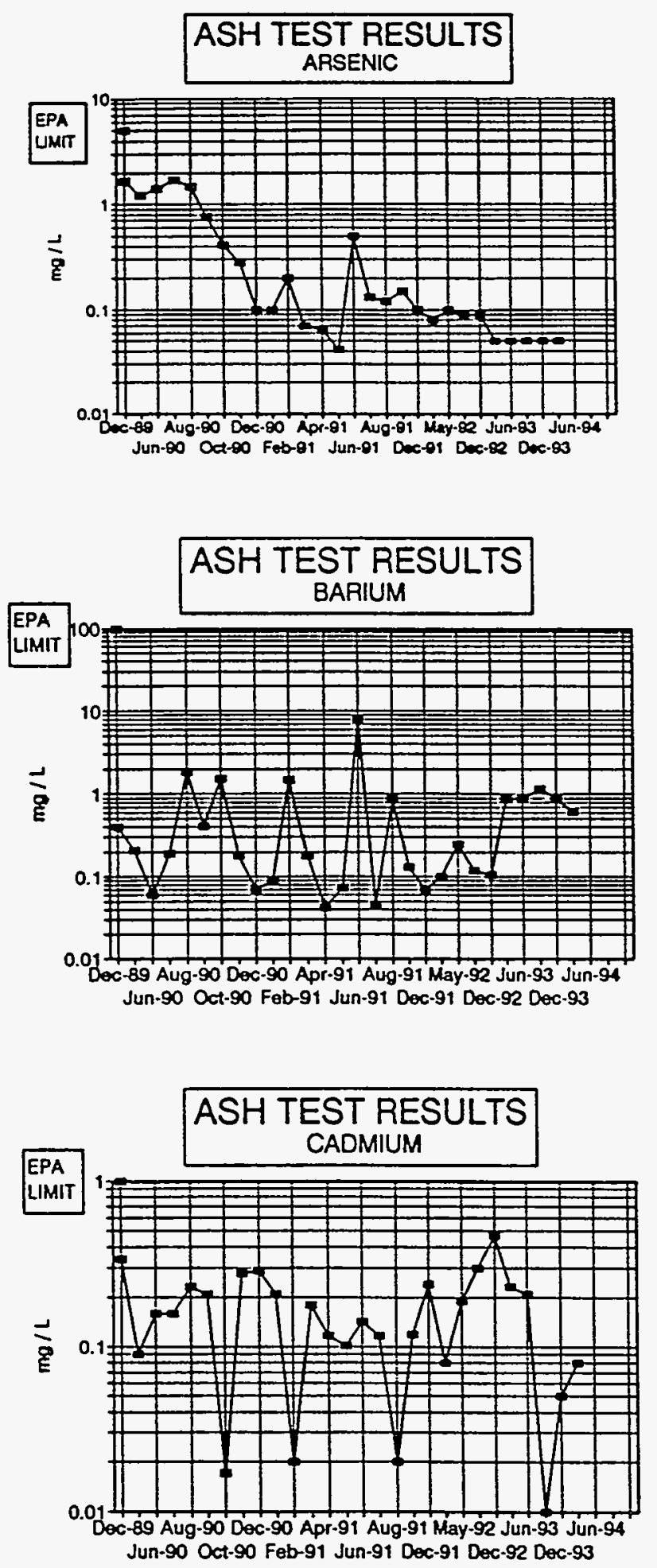
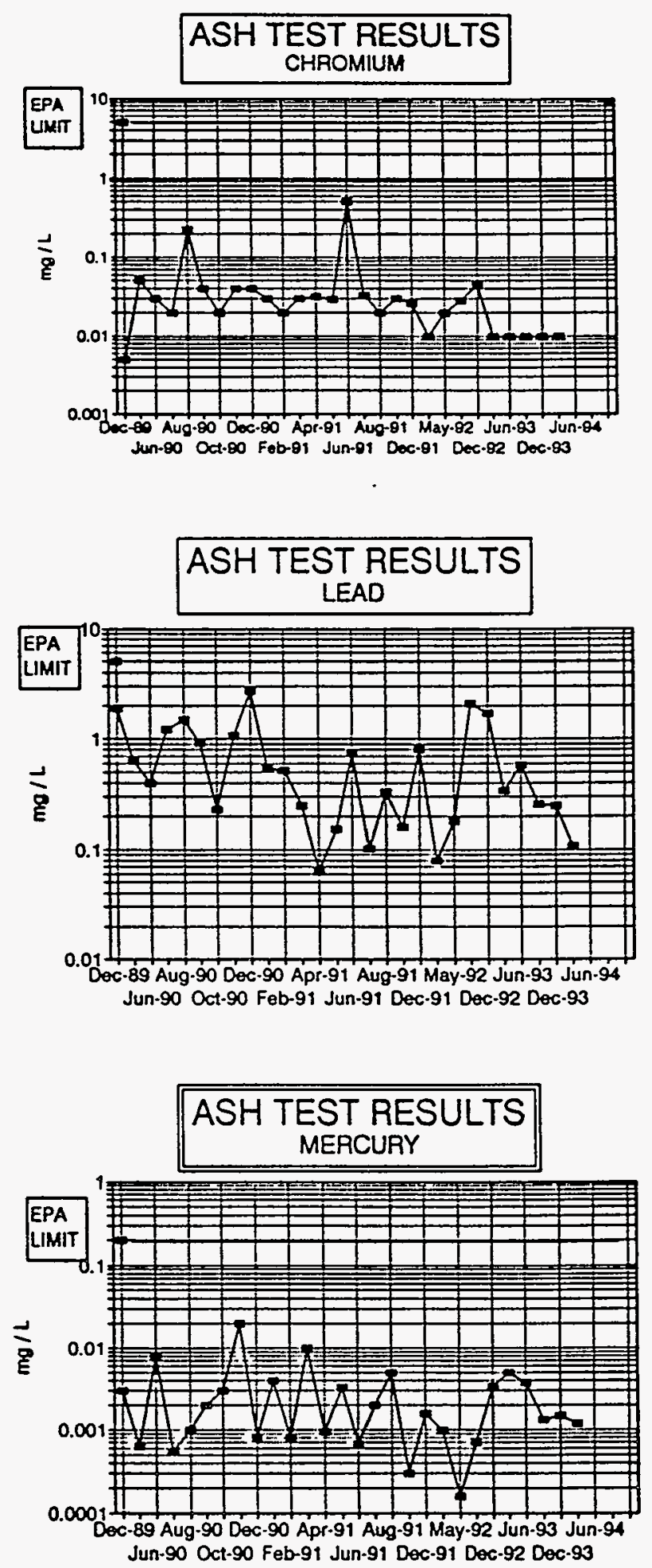

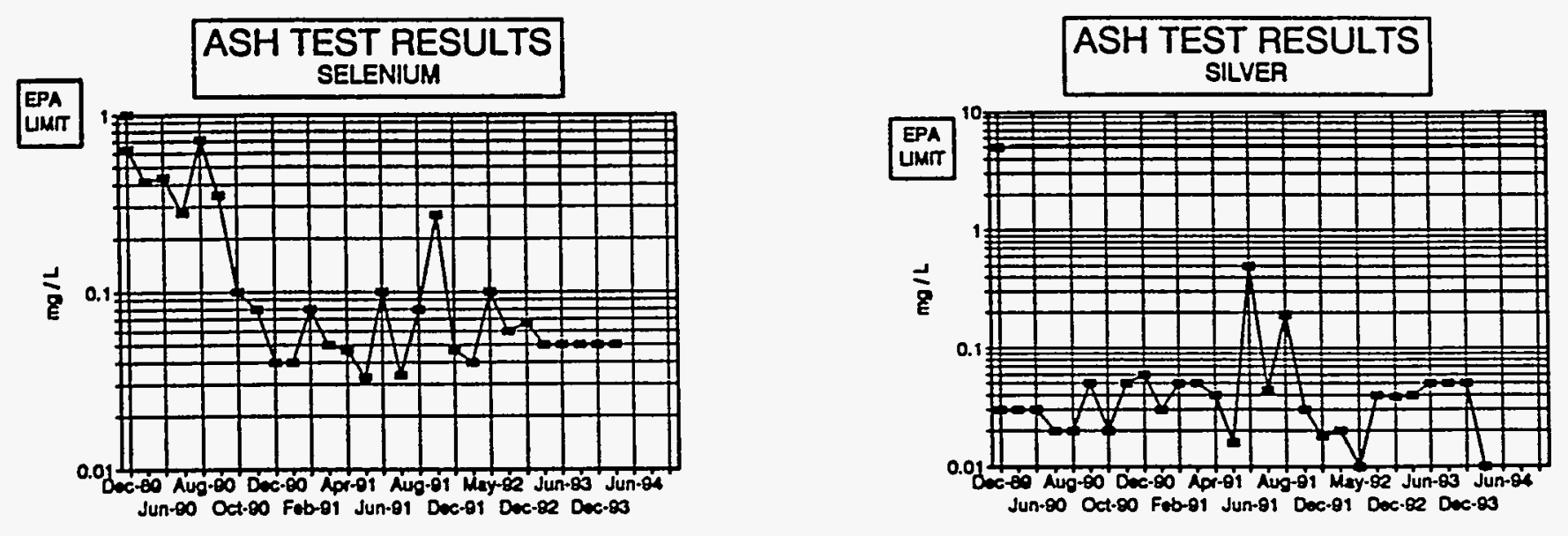
Appendix B1

Sieve Analysis Results

\section{Introduction}

The sieve analyses were included in order to classify the various ash types as a possible aggregate source. Three ash types were tested: H-POWER processed bottom ash, H-POWER combined ash, and Waipahu Incinerator ash. The Waipahu ash used herein was first run through the BAMR at H-POWER. Both Hawaiian Bitumuls and Grace Pacific were instrumental in performing the analyses. Data was compiled from both sources and is presented in this appendix. The following U.S. Standard sieve sizes were used:

$\begin{array}{lll}3 / 4 "(19.05 \mathrm{~mm}) & 1 / 2 "(12.7 \mathrm{~mm}) & 3 / 8 "(9.53 \mathrm{~mm}) \\ \# 4(4.75 \mathrm{~mm}) & \# 8(2.36 \mathrm{~mm}) & \# 16(1.18 \mathrm{~mm}) \\ \# 30(0.60 \mathrm{~mm}) & \# 50(0.30 \mathrm{~mm}) & \# 100(0.15 \mathrm{~mm}) \\ \# 200(0.075 \mathrm{~mm}) & & \end{array}$

Recognizing that there are many different conventions for reporting particle size distributions, we have made an effort to use whichever method seemed to best characterize the material. In most cases, the data is reported as the percent retained on each particular screen size. This was done primarily to aid in the qualitative analysis of the ash, and also in anticipation of being able to use only selected size fractions. Whenever possible, the percent passing (or percent finer) is also. reported.

\section{Results}

Table 1 contains a summary of the entire sieve analysis. The top of the table contains the individual trials along with the averages, reported as percent retained. The cumulative percent retained from (\#16 and below) is also included as this size fraction seemed to be the best suited for the anticipated use. The bottom of the table includes only the average gradations for the ash types, but reported as percent passing each screen size.

Tables 2-7 contain the original sieve analysis data (two sets for each type), and Figures 1,2, and 3 are their respective graphical representations.

Tables 8-12 contain sieve data pertaining only to H-POWER combined ash. These tests were included to determine what effect 
dry mixing would have on the overall gradation. As the results in Figure 4 indicate, the "normally-mixed" and then oven-dried ash showed a considerably smaller amount of fine material (-\#100), than did the samples which were dried prior to mixing. This is believed to be a result of the fly ash pelletizing upon mixing with the wet bottom ash. 


\section{SIEVE ANALYSIS Summary}

\begin{tabular}{|c|c|c|c|c|c|c|c|c|c|c|c|c|}
\hline & \multicolumn{11}{|c|}{ Sieve Size Distribution (\% Retained) } & \multirow[b]{2}{*}{ Cum. \% Retained } \\
\hline U.S.S. Sieve Size & 314 & 112 & 318 & $\# 4$ & $\# 8$ & $\# 16$ & $\# 30$ & $\# 50$ & $\# 100$ & $\# 200$ & $<\# 200$ & \\
\hline H-POWER Bottom Ash - Trial 1 & $0.00 \%$ & $3.33 \%$ & $3.90 \%$ & $17.75 \%$ & $27.15 \%$ & $23.33 \%$ & $11.09 \%$ & $5.63 \%$ & $3.24 \%$ & $2.46 \%$ & $2.12 \%$ & $47.87 \%$ \\
\hline H-POWER Bottom Ash - Average & $1.10 \%$ & $3.82 \%$ & $4.12 \%$ & $15.74 \%$ & $27.18 \%$ & $24.47 \%$ & $10.71 \%$ & $5.57 \%$ & $3.25 \%$ & $2.05 \%$ & $2.02 \%$ & $48.06 \%$ \\
\hline H-POWER Combined Ash - Trial 1 & $0.15 \%$ & $3.15 \%$ & $3.37 \%$ & $15.45 \%$ & $26.66 \%$ & $19.21 \%$ & $10.37 \%$ & $8.66 \%$ & $7.26 \%$ & $3.61 \%$ & $2.10 \%$ & $51.21 \%$ \\
\hline Waipahu Combined Ash - Trial 1 & $1.52 \%$ & $11.35 \%$ & $11.39 \%$ & $24.21 \%$ & $15.82 \%$ & $10.06 \%$ & $6.67 \%$ & $5.63 \%$ & $5.23 \%$ & $7.06 \%$ & $1.05 \%$ & $35.70 \%$ \\
\hline Waipahu Combined Ash - Trial 2 & $2.28 \%$ & $9.03 \%$ & $12.18 \%$ & $16.07 \%$ & $15.09 \%$ & $12.90 \%$ & $9.75 \%$ & $7.75 \%$ & $6.61 \%$ & $3.79 \%$ & $4.55 \%$ & $45.35 \%$ \\
\hline Waipahu Combined Ash - Average & $1.90 \%$ & $10.19 \%$ & $11.79 \%$ & $20.14 \%$ & $15.46 \%$ & $11.48 \%$ & $8.21 \%$ & $6.69 \%$ & $5.92 \%$ & $5.43 \%$ & $2.80 \%$ & $40.53 \%$ \\
\hline
\end{tabular}

\begin{tabular}{|c|c|c|c|c|c|c|c|c|c|c|c|}
\cline { 2 - 10 } \multicolumn{1}{c|}{} & \multicolumn{9}{c|}{ Sieve Size Distribution (\% Passing) } \\
\hline U.S.S. Sieve Size & 314 & 112 & 318 & $\# 4$ & $\# 8$ & $\# 16$ & $\# 30$ & $\# 50$ & $\# 100$ & $\# 200$ & $<\# 200$ \\
\hline Sieve Size (mm) & 19.00 & 12.70 & 9.53 & 4.75 & 2.36 & 1.18 & 0.60 & 0.30 & 0.15 & 0.075 & $<0.075$ \\
\hline H-POWER Bottom Ash - Average & $98.90 \%$ & $95.09 \%$ & $90.97 \%$ & $75.23 \%$ & $48.06 \%$ & $23.59 \%$ & $12.88 \%$ & $7.31 \%$ & $4.06 \%$ & $2.01 \%$ & $0.00 \%$ \\
\hline H-POWER Combined Ash - Average & $99.56 \%$ & $97.49 \%$ & $94.39 \%$ & $78.87 \%$ & $52.73 \%$ & $32.55 \%$ & $21.80 \%$ & $12.58 \%$ & $5.31 \%$ & $1.84 \%$ & $0.00 \%$ \\
\hline Waipahu Combined Ash - Average & $98.10 \%$ & $87.91 \%$ & $76.13 \%$ & $55.99 \%$ & $40.53 \%$ & $29.05 \%$ & $20.84 \%$ & $14.15 \%$ & $8.23 \%$ & $2.80 \%$ & $0.00 \%$ \\
\hline
\end{tabular}




\section{SIEVE ANALYSIS H-POWER Bottom Ash \\ Trial 1}

\begin{tabular}{|c|r|r|r|r|}
\hline Size & Weight (g) & \% Retained & Cum \% Ret. & \% Passing \\
\hline $3 / 4$ & 0 & $0.00 \%$ & $0.00 \%$ & $100.00 \%$ \\
\hline $1 / 2$ & 128.2 & $3.33 \%$ & $3.33 \%$ & $96.67 \%$ \\
\hline $3 / 8$ & 150.3 & $3.90 \%$ & $7.23 \%$ & $92.77 \%$ \\
\hline 4 & 683.7 & $17.75 \%$ & $24.98 \%$ & $75.02 \%$ \\
\hline 8 & 1045.8 & $27.15 \%$ & $52.14 \%$ & $47.86 \%$ \\
\hline 16 & 898.5 & $23.33 \%$ & $75.47 \%$ & $24.53 \%$ \\
\hline 30 & 427 & $11.09 \%$ & $86.55 \%$ & $13.45 \%$ \\
\hline 50 & 216.9 & $5.63 \%$ & $92.18 \%$ & $7.82 \%$ \\
\hline 100 & 124.8 & $3.24 \%$ & $95.43 \%$ & $4.57 \%$ \\
\hline 200 & 94.7 & $2.46 \%$ & $97.88 \%$ & $2.12 \%$ \\
\hline-200 & 81.5 & $2.12 \%$ & $100.00 \%$ & $0.00 \%$ \\
\hline Total & 3851.4 & & & \\
\hline F.M & 4.37 & & & \\
\hline
\end{tabular}

Table 2 


\section{SIEVE ANALYSIS \\ H-POWER Bottom Ash \\ Trial 2}

\begin{tabular}{|c|r|r|r|r|}
\hline Size & Weight $(\mathrm{g})$ & $\%$ Retained & Cum \% Ret. & P Passing \\
\hline $3 / 4$ & 60.7 & $2.20 \%$ & $2.20 \%$ & $97.80 \%$ \\
\hline $1 / 2$ & 118.6 & $4.30 \%$ & $6.50 \%$ & $93.50 \%$ \\
\hline $3 / 8$ & 119.7 & $4.34 \%$ & $10.83 \%$ & $89.17 \%$ \\
\hline 4 & 378.7 & $13.72 \%$ & $24.55 \%$ & $75.45 \%$ \\
\hline 8 & 750.8 & $27.20 \%$ & $51.75 \%$ & $48.25 \%$ \\
\hline 16 & 706.7 & $25.60 \%$ & $77.35 \%$ & $22.65 \%$ \\
\hline 30 & 285.1 & $10.33 \%$ & $87.68 \%$ & $12.32 \%$ \\
\hline 50 & 152.2 & $5.51 \%$ & $93.20 \%$ & $6.80 \%$ \\
\hline 100 & 89.6 & $3.25 \%$ & $96.44 \%$ & $3.56 \%$ \\
\hline 200 & 45.4 & $1.64 \%$ & $98.09 \%$ & $1.91 \%$ \\
\hline-200 & 52.8 & $1.91 \%$ & $100.00 \%$ & $0.00 \%$ \\
\hline Total & 2760.3 & & &
\end{tabular}

Table 3 


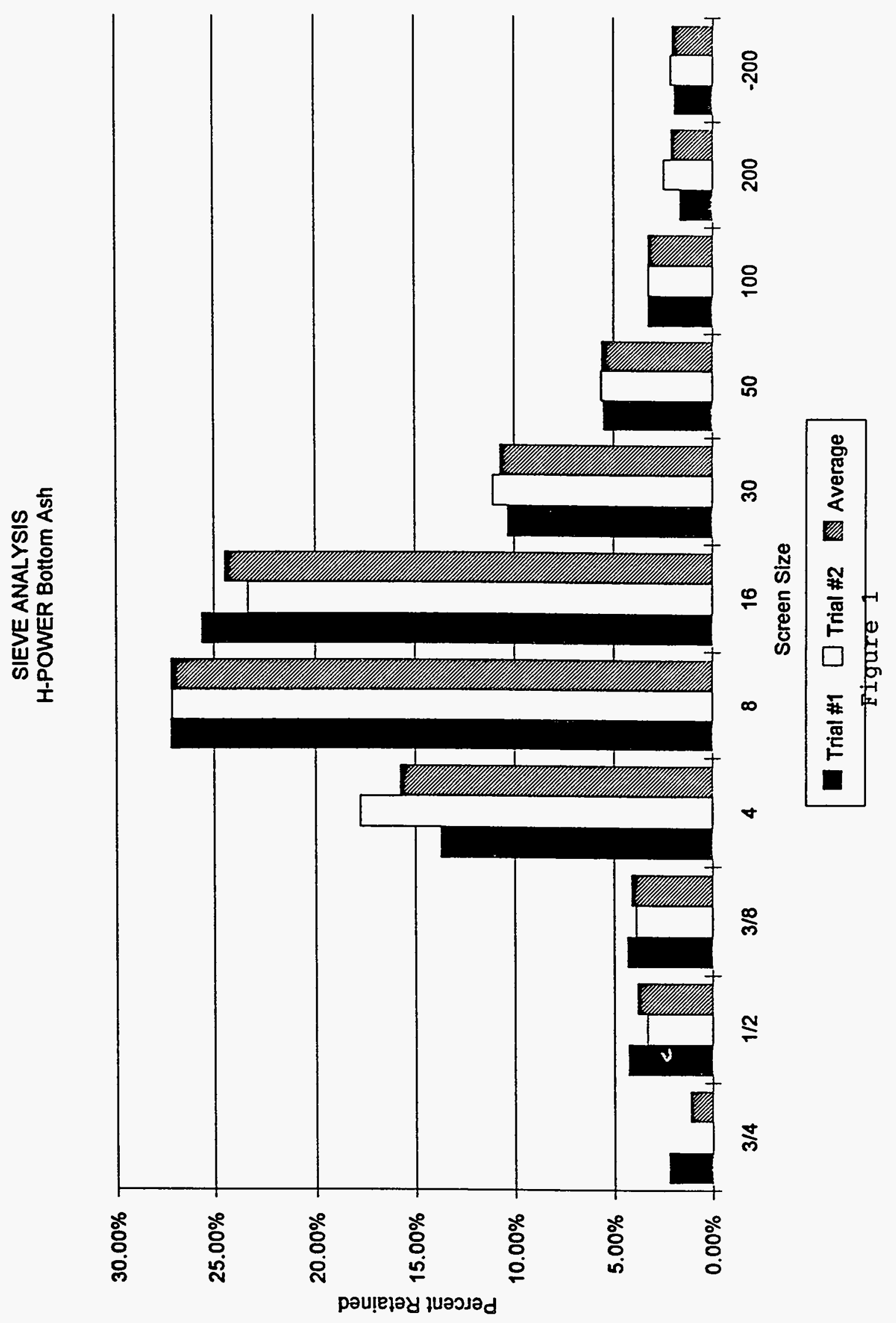

B1-6 


\section{SIEVE ANALYSIS \\ H-POWER Combined Ash \\ Trial 1}

\begin{tabular}{|c|c|c|c|c|}
\hline Size & Weight (g) & $\%$ Retained & Cum \% Ret. & $\%$ Passing \\
\hline $3 / 4$ & 4.4 & $0.15 \%$ & $0.15 \%$ & $99.85 \%$ \\
\hline $1 / 2$ & 93.2 & $3.15 \%$ & $3.30 \%$ & $96.70 \%$ \\
\hline $3 / 8$ & 99.8 & $3.37 \%$ & $6.67 \%$ & $93.33 \%$ \\
\hline 4 & 456.9 & $15.45 \%$ & $22.12 \%$ & $77.88 \%$ \\
\hline 8 & 788.6 & $26.66 \%$ & $48.79 \%$ & $51.21 \%$ \\
\hline 16 & 568.1 & $19.21 \%$ & $68.00 \%$ & $32.00 \%$ \\
\hline 30 & 306.6 & $10.37 \%$ & $78.36 \%$ & $21.64 \%$ \\
\hline 50 & 256.2 & $8.66 \%$ & $87.03 \%$ & $12.97 \%$ \\
\hline 100 & 214.8 & $7.26 \%$ & $94.29 \%$ & $5.71 \%$ \\
\hline 200 & 106.8 & $3.61 \%$ & $97.90 \%$ & $2.10 \%$ \\
\hline-200 & 62.1 & $2.10 \%$ & $100.00 \%$ & $0.00 \%$ \\
\hline Total & 2957.5 & & & ? \\
\hline F.M & 4.09 & & 11:19:1:1:19: & $4:=$ \\
\hline
\end{tabular}

Table 4 


\section{SIEVE ANALYSIS \\ H-POWER Combined Ash \\ Trial 2}

\begin{tabular}{|c|c|c|c|c|}
\hline Size & Weight (g) & $\%$ Retained & Cum \% Ret. & $\%$ Passing \\
\hline $3 / 4$ & 41 & $0.74 \%$ & $0.74 \%$ & $99.26 \%$ \\
\hline $1 / 2$ & 55.1 & $0.99 \%$ & $1.73 \%$ & $98.27 \%$ \\
\hline $3 / 8$ & 156.6 & $2.82 \%$ & $4.55 \%$ & $95.45 \%$ \\
\hline 4 & 866.4 & $15.59 \%$ & $20.13 \%$ & $79.87 \%$ \\
\hline 8 & 1424.5 & $25.63 \%$ & $45.76 \%$ & $54.24 \%$ \\
\hline 16 & 1175.8 & $21.15 \%$ & $66.91 \%$ & $33.09 \%$ \\
\hline 30 & 618.2 & $11.12 \%$ & $78.03 \%$ & $21.97 \%$ \\
\hline 50 & 544.1 & $9.79 \%$ & $87.82 \%$ & $12.18 \%$ \\
\hline 100 & 404.1 & $7.27 \%$ & $95.09 \%$ & $4.91 \%$ \\
\hline 200 & 185.1 & $3.33 \%$ & $98.42 \%$ & $1.58 \%$ \\
\hline-200 & 88.1 & $1.58 \%$ & $100.00 \%$ & $0.00 \%$ \\
\hline Total & 5559 & 4.9. & 18 & \\
\hline F.M & 4.01 & & & \\
\hline
\end{tabular}

Table 5 
SIEVE ANALYSIS

H-POWER Mixed Ash

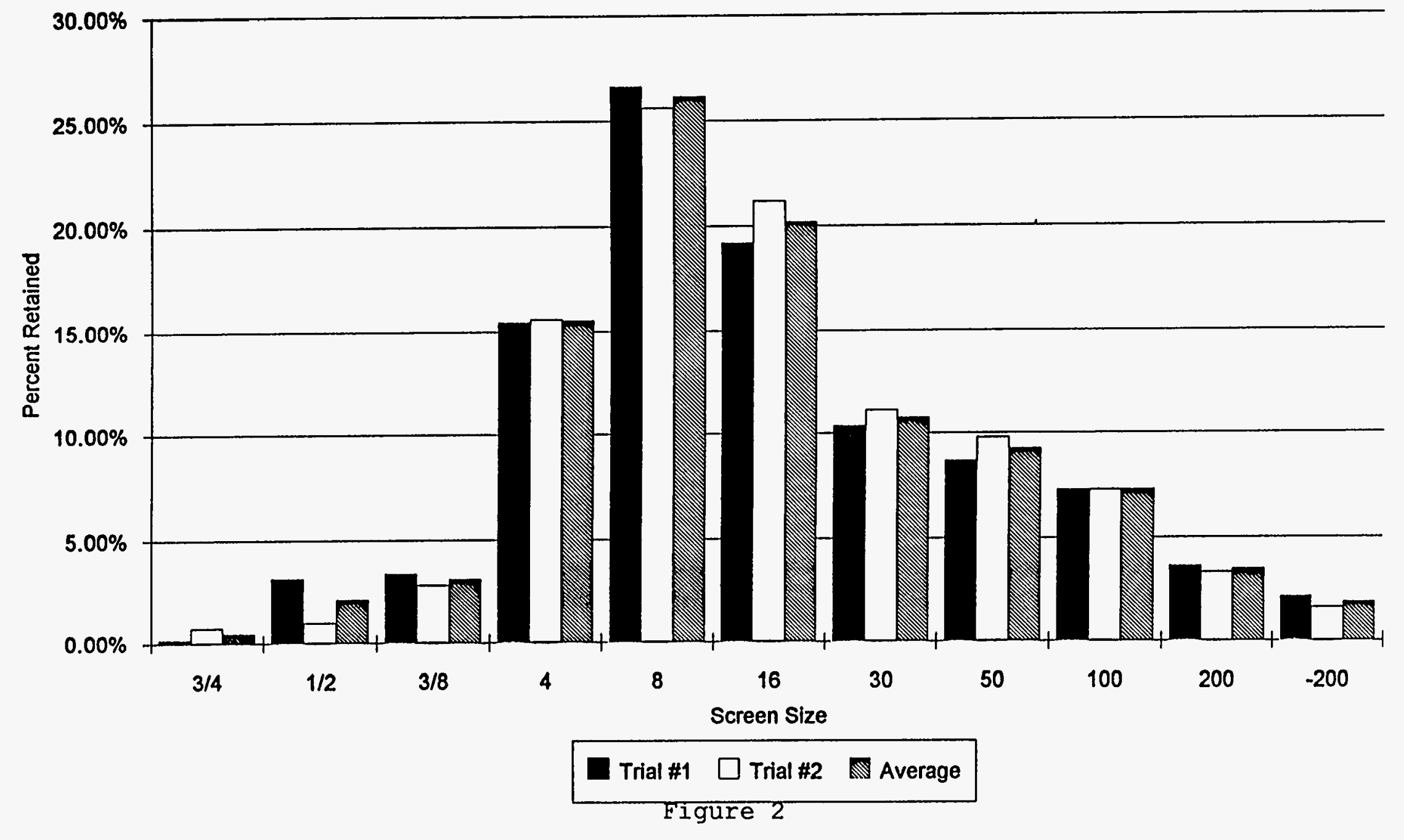




\section{SIEVE ANALYSIS \\ Waipahu Combined Ash \\ Trial 1}

\begin{tabular}{|c|r|r|r|r|}
\hline Size & Weight $(\mathrm{g})$ & $\%$ Retained & Cum \% Ret. & $\%$ Passing \\
\hline $3 / 4$ & 56.3 & $1.52 \%$ & $1.52 \%$ & $98.48 \%$ \\
\hline $1 / 2$ & 419.7 & $11.35 \%$ & $12.87 \%$ & $87.13 \%$ \\
\hline $3 / 8$ & 421.2 & $11.39 \%$ & $24.27 \%$ & $75.73 \%$ \\
\hline 4 & 895 & $24.21 \%$ & $48.47 \%$ & $51.53 \%$ \\
\hline 8 & 584.9 & $15.82 \%$ & $64.29 \%$ & $35.71 \%$ \\
\hline 16 & 372 & $10.06 \%$ & $74.36 \%$ & $25.64 \%$ \\
\hline 30 & 246.5 & $6.67 \%$ & $81.02 \%$ & $18.98 \%$ \\
\hline 50 & 208 & $5.63 \%$ & $86.65 \%$ & $13.35 \%$ \\
\hline 100 & 193.4 & $5.23 \%$ & $91.88 \%$ & $8.12 \%$ \\
\hline 200 & 261.2 & $7.06 \%$ & $98.95 \%$ & $1.05 \%$ \\
\hline-200 & 39 & $1.05 \%$ & $100.00 \%$ & $0.00 \%$ \\
\hline Total & 3697.2 & & & \\
\hline F.M & 4.85 & & & \\
\hline
\end{tabular}

Table 6 


\section{SIEVE ANALYSIS \\ Waipahu Combined Ash \\ Trial 2}

\begin{tabular}{|r|r|r|r|r|}
\hline Size & Weight $(\mathrm{g})$ & $\%$ Retained & Cum \% Ret. & $\%$ Passing \\
\hline $3 / 4$ & 50.3 & $2.28 \%$ & $2.28 \%$ & $97.72 \%$ \\
\hline $1 / 2$ & 199.3 & $9.03 \%$ & $11.31 \%$ & $88.69 \%$ \\
\hline $3 / 8$ & 268.8 & $12.18 \%$ & $23.50 \%$ & $76.50 \%$ \\
\hline 4 & 354.4 & $16.07 \%$ & $39.56 \%$ & $60.44 \%$ \\
\hline 8 & 332.9 & $15.09 \%$ & $54.66 \%$ & $45.34 \%$ \\
\hline 16 & 284.6 & $12.90 \%$ & $67.56 \%$ & $32.44 \%$ \\
\hline 30 & 215.1 & $9.75 \%$ & $77.31 \%$ & $22.69 \%$ \\
\hline 50 & 170.9 & $7.75 \%$ & $85.05 \%$ & $14.95 \%$ \\
\hline 100 & 145.8 & $6.61 \%$ & $91.66 \%$ & $8.34 \%$ \\
\hline 200 & 83.6 & $3.79 \%$ & $95.45 \%$ & $4.55 \%$ \\
\hline-200 & 100.3 & $4.55 \%$ & $100.00 \%$ & $0.00 \%$ \\
\hline Total & 2206 & & & \\
\hline F.M & 4.53 & & & \\
\hline
\end{tabular}


SIEVE ANALYSIS

Waipahu Ash

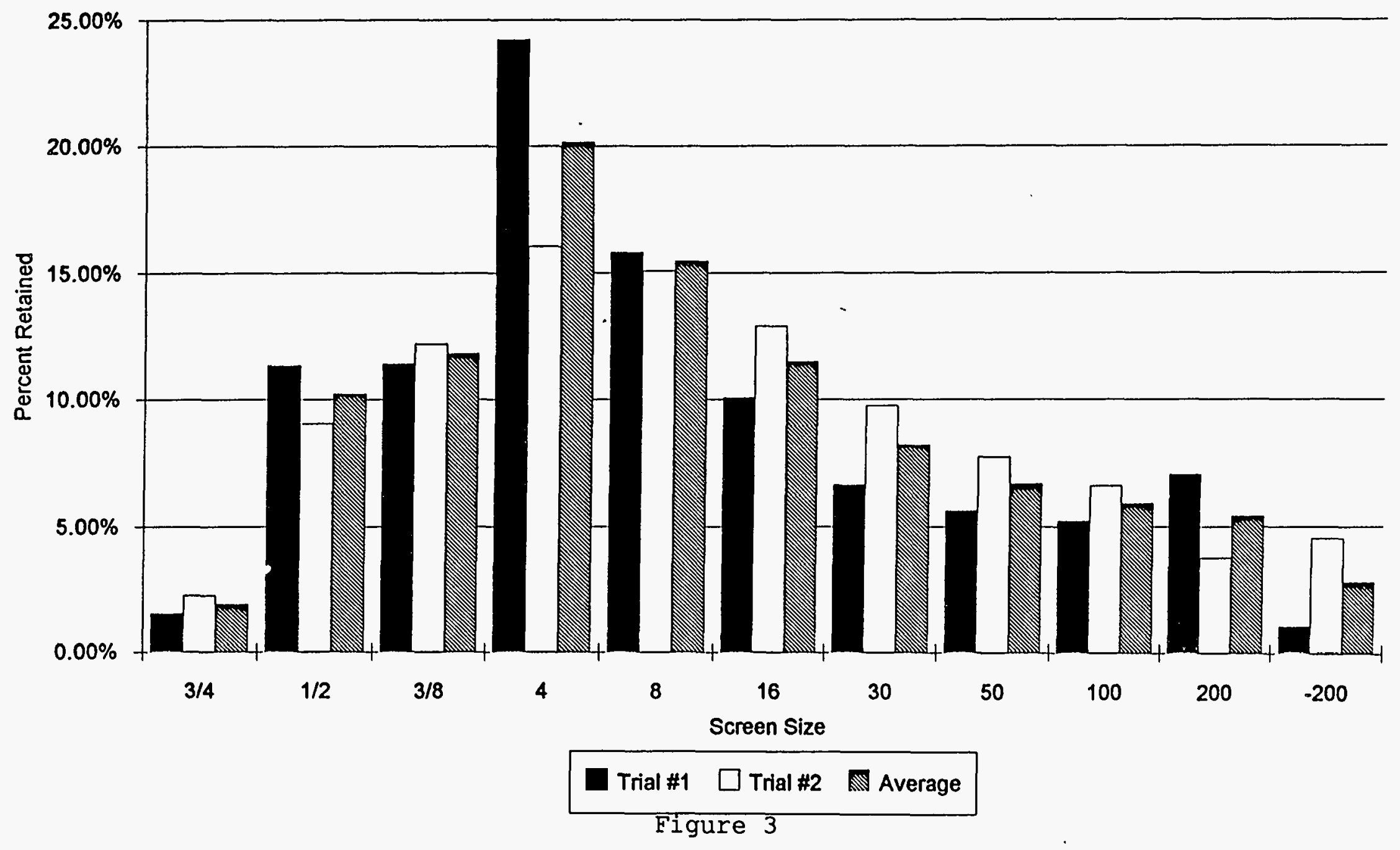




\section{SIEVE SIZE ANALYSIS \\ H-POWER Combined Ash}

TEST DATE

7-Dec-93

\begin{tabular}{|c|c|c|}
\hline $\begin{array}{c}\text { SCREEN } \\
\text { SIZE }\end{array}$ & $\begin{array}{c}\text { WEIGHT } \\
(\mathrm{g})\end{array}$ & $\begin{array}{c}\text { PERCENT } \\
\text { OF TOTAL }\end{array}$ \\
\hline & & \\
\hline $3 / 4$ & 55.00 & $0.83 \%$ \\
\hline $1 / 2$ & 110.00 & $1.66 \%$ \\
\hline $3 / 8$ & 205.00 & $3.09 \%$ \\
\hline 4 & 950.00 & $14.32 \%$ \\
\hline 8 & 1640.00 & $24.72 \%$ \\
\hline 30 & 2750.00 & $41.45 \%$ \\
\hline 100 & 845.00 & $12.74 \%$ \\
\hline 200 & 75.00 & $1.13 \%$ \\
\hline-200 & 5.00 & $0.08 \%$ \\
\hline TOTAL & 6635 & $100.00 \%$ \\
\hline
\end{tabular}

Table 8 


\section{SIEVE SIZE ANALYSIS \\ H-POWER Combined Ash OVEN-DRIED.}

TEST DATE

18-Dec-93

\begin{tabular}{|c|c|c|}
\hline $\begin{array}{c}\text { SCREEN } \\
\text { SIZE }\end{array}$ & $\begin{array}{c}\text { WEIGHT } \\
(\mathrm{g})\end{array}$ & $\begin{array}{c}\text { PERCENT } \\
\text { OF TOTAL }\end{array}$ \\
\hline & & \\
\hline $3 / 4$ & 41.00 & $0.74 \%$ \\
\hline $1 / 2$ & 55.10 & $0.99 \%$ \\
\hline $3 / 8$ & 156.60 & $2.82 \%$ \\
\hline 4 & 866.40 & $15.59 \%$ \\
\hline 8 & 1424.50 & $25.63 \%$ \\
\hline 30 & 1794.00 & $32.27 \%$ \\
\hline 100 & 948.20 & $17.06 \%$ \\
\hline 200 & 185.10 & $3.33 \%$ \\
\hline-200 & 88.10 & $1.58 \%$ \\
\hline TOTAL & 5559 & $100.00 \%$ \\
\hline
\end{tabular}

Table 9 


\section{SIEVE SIZE ANALYSIS \\ H-POWER Combined Ash \\ MIXED DRY}

Trial 1

TEST DATE

18-Dec-93

\begin{tabular}{|c|c|c|}
\hline $\begin{array}{c}\text { SCREEN } \\
\text { SIZE }\end{array}$ & $\begin{array}{c}\text { WEIGHT } \\
(\mathrm{g})\end{array}$ & $\begin{array}{c}\text { PERCENT } \\
\text { OF TOTAL }\end{array}$ \\
\hline & & \\
\hline $3 / 4$ & 0.00 & $0.00 \%$ \\
\hline $1 / 2$ & 32.30 & $1.76 \%$ \\
\hline $3 / 8$ & 59.60 & $3.26 \%$ \\
\hline 4 & 219.60 & $12.00 \%$ \\
\hline 8 & 330.10 & $18.03 \%$ \\
\hline 30 & 497.80 & $27.19 \%$ \\
\hline 100 & 299.20 & $16.35 \%$ \\
\hline 200 & 221.40 & $12.10 \%$ \\
\hline-200 & 170.50 & $9.31 \%$ \\
\hline TOTAL & 1830.5 & $100.00 \%$ \\
\hline
\end{tabular}

Table 10 


\section{SIEVE SIZE ANALYSIS \\ H-POWER Combined Ash \\ MIXED DRY}

Trial 2

TEST DATE

18-Dec-93

\begin{tabular}{|c|c|c|}
\hline $\begin{array}{c}\text { SCREEN } \\
\text { SIZE }\end{array}$ & $\begin{array}{c}\text { WEIGHT } \\
(\mathrm{g})\end{array}$ & $\begin{array}{c}\text { PERCENT } \\
\text { OF TOTAL }\end{array}$ \\
\hline $3 / 4$ & & \\
\hline $1 / 2$ & 0.00 & $0.00 \%$ \\
\hline $3 / 8$ & 35.30 & $1.60 \%$ \\
\hline 4 & 61.30 & $2.78 \%$ \\
\hline 8 & 270.00 & $12.25 \%$ \\
\hline 30 & 368.70 & $16.72 \%$ \\
\hline 100 & 534.80 & $24.26 \%$ \\
\hline 200 & 371.80 & $16.87 \%$ \\
\hline-200 & 340.00 & $15.42 \%$ \\
\hline TOTAL & 222.60 & $10.10 \%$ \\
\hline & 2204.5 & $100.00 \%$ \\
\hline
\end{tabular}

Table 11 


\section{SIEVE SIZE ANALYSIS \\ H-POWER Combined Ash \\ MIXED DRY \\ TOTAL}

TEST DATE

18-Dec-93

\begin{tabular}{|c|c|c|}
\hline $\begin{array}{c}\text { SCREEN } \\
\text { SIZE }\end{array}$ & $\begin{array}{c}\text { WEIGHT } \\
(\mathrm{g})\end{array}$ & $\begin{array}{c}\text { PERCENT } \\
\text { OF TOTAL }\end{array}$ \\
\hline & & \\
\hline $3 / 4$ & 0.00 & $0.00 \%$ \\
\hline $1 / 2$ & 67.60 & $1.68 \%$ \\
\hline $3 / 8$ & 120.90 & $3.00 \%$ \\
\hline 4 & 489.60 & $12.13 \%$ \\
\hline 8 & 698.80 & $17.32 \%$ \\
\hline 30 & 1032.60 & $25.59 \%$ \\
\hline 100 & 671.00 & $16.63 \%$ \\
\hline 200 & 561.40 & $13.91 \%$ \\
\hline-200 & 393.10 & $9.74 \%$ \\
\hline TOTAL & 4035 & $100.00 \%$ \\
\hline
\end{tabular}

Table 12 


\section{SIEVE SIZE ANALYSIS \\ H-POWER Combined Ash \\ MIXED DRY}

AVERAGE

TEST DATE

18-Dec-93

\begin{tabular}{|c|c|c|c|}
\hline $\begin{array}{c}\text { SCREEN } \\
\text { SIZE }\end{array}$ & $\begin{array}{c}\text { WEIGHT } \\
(\mathrm{g})\end{array}$ & $\begin{array}{c}\text { PERCENT } \\
\text { OF TOTAL }\end{array}$ & $\begin{array}{c}\text { STANDARD } \\
\text { DEVIATION }\end{array}$ \\
\hline & & $\%$ & \\
\hline $3 / 4$ & 0.00 & $0.00 \%$ & $0.00 \%$ \\
\hline $1 / 2$ & 33.80 & $1.68 \%$ & $0.05 \%$ \\
\hline $3 / 8$ & 60.45 & $3.00 \%$ & $0.15 \%$ \\
\hline 4 & 244.80 & $12.13 \%$ & $0.08 \%$ \\
\hline 8 & 349.40 & $17.32 \%$ & $0.42 \%$ \\
\hline 30 & 516.30 & $25.59 \%$ & $0.94 \%$ \\
\hline 100 & 335.50 & $16.63 \%$ & $0.17 \%$ \\
\hline 200 & 280.70 & $13.91 \%$ & $1.07 \%$ \\
\hline-200 & 196.55 & $9.74 \%$ & $0.25 \%$ \\
\hline TOTAL & 2017.5 & $100.00 \%$ & \\
\hline
\end{tabular}

Table 13 
SIEVE ANALYSIS

H-POWER Mixed Ash

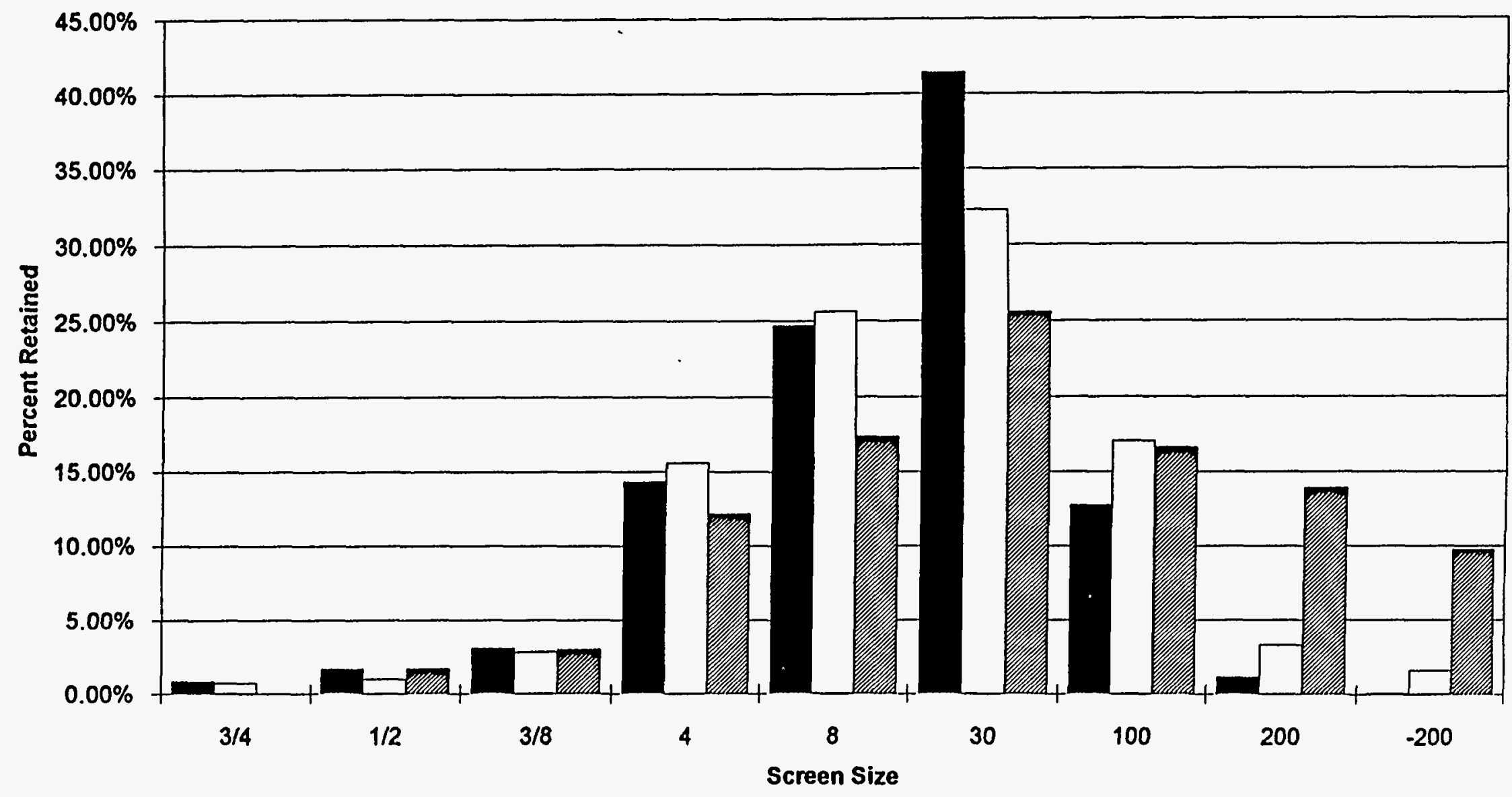

Undried Sample $\square$ Oven-dried Sample Mixed Dry 
Appendix B2

\section{Chemical Analysis of Screened Ash Fractions}

Task 2 chemical analysis was carried out following the methods listed below:

\section{Chemical Analysis Methods}

$\begin{array}{ll}\text { Aluminum } & \text { EPA Method } 3050 \mathrm{M} \\ \text { Cadmium } & \text { EPA Method } 6010 \\ \text { Calcium } & \text { EPA Method } 6010 \\ \text { Copper } & \text { EPA Method } 6010 \\ \text { Iron } & \text { EPA Method } 6010 \\ \text { Lead } & \text { EPA Method } 6010 \\ \text { Mercury } & \text { EPA Method } 6010 \\ \text { Silicon } & \text { EPA Method } 6010 \\ \text { Zinc } & \text { EPA Method } 6010 \\ \text { pH } & \text { EPA Method } 9045 \\ \text { Chloride } & \text { EPA Method } 325.3 \\ \text { Sulfate } & \text { EPA Method } 375.4 \\ \text { Moisture } & \text { CLP ILM 2.0 }\end{array}$

\section{Sumnary Findings}

The following is a detailed summary of the Task 2 chemical analysis findings. Please refer to the appropriate tables listed at the beginning of each section. Data appearing in the tables has been adapted from the E.L. Pacific laboratory report, which can be found beginning on page B2-6 of this appendix.

Aluminum through Chromium Refer to Table 1 on page $\mathrm{B} 2-3$

Aluminum - Average: 43,428 PPM. This is slightly higher than the 40,000 PPM average for aluminum found in the Task 1 ash mixture analyses. A possible explanation for the difference is that the average in Task 2 only included the minus \#4 ash fraction. About $15 \%$ of the ash included in the Task 1 mixture analyses, was in size fractions larger than were included in the Task 2 analysis. In general it is thought metals would tend to be concentrated more in the smaller size fractions. However, the results for both H-POWER and Waipahu combined ash show that highest concentration (83-86,000 PPM) was in the \#8 size material, and was almost twice the concentration of the minus \#200 
material (41-50,000 PPM.) overall, the results show very uniform distribution over the various size fractions. This is considered a positive result because if the smaller size fractions are the main source for aggregate, there is no evidence that aluminum or many of the other metals are more concentrated in these smaller fractions.

Calcium - Average: 70,953 PPM. The H-POWER combined ash concentrations averaged 109,423 PPM compared to 42,900 PPM for the Waipahu ash. The difference is due to the lime scrubber system at H-POWER. The concentrations found in the Task 2 analysis are consistent with the Task 1 results. There is, however, some enrichment in the \#200 and smaller size fractions.

Cadmium - Average: 18 PPM. This is similar to the average for H-POWER fly and bottom ash found in the Task 1 analysis (2.5-26.5 PPM). There is some evidence of enrichment of cadmium in the minus \#200 size fraction. Otherwise, the distribution appears consistent among all size fractions for all ash types.

Copper - Average: 6,109 PPM. This is much higher than that found in the Task 1 copper analysis $(1,886 \mathrm{PPM})$. There is greater variability evident among the various size fractions than most of the other metals. Because of this variability and the small population of analyses, it is difficult to see any particular trends for this metal among the 3 types of ash or among the different size fractions. Copper levels in landfill soil samples averaged 98 PPM.

Iron - Average: 22,570 PPM. As with the other metals, the variability in iron concentrations is fairly consistent around the average with no evidence of enrichment in the smaller ash sizes. Moreover, Waipahu's enrichment--with one exception--is in the larger size fractions. Also note that the iron levels in local soil samples averaged 74,995 PPM.

Lead - Average: 3,547 PPM. Once again, the variability is not considered significant. Except for one high value (25,000 PPM for the \#8 H-POWER bottom ash), all other size fractions and other ash types had fairly consistent concentrations around 2-3,000 PPM. There was no evidence of enrichment in the smaller sizes.

Mercury - The mercury elemental analysis averaged just slightly above the detection level of 0.2 PPM for two of the 
ash types. In fact 13 of the 21 samples analyzed were below mercury detection levels. While the H-POWER bottom ash and Waipahu ash were essentially at or below detection levels, there was evidence of mercury build up on the \#100 and smaller size fractions of the H-POWER combined ash. These levels were still about the same as found in the soil control samples examined in Task 1 mixture

Silicon - Average: 106,730 PPM. The silicon concentrations were quite consistent among the 3 ash types and among the ash size fractions. The levels also compare with results from the soil control samples from Task 1.

\begin{tabular}{|c|c|c|c|c|c|c|c|c|c|}
\hline \multicolumn{10}{|c|}{ Chemical Analysis in units of PPM unless otherwise indicated } \\
\hline$\%$ In size fraction & Ash Type/Size & Al & Ca & Cd & Cu & $F_{\bullet}$ & $\overline{\mathbf{P b}}$ & $\mathrm{Hg}$ & Si \\
\hline & HFOWER combined & & & & & & & & \\
\hline $26.70 \%$ & B & 83,000 & 110,000 & 9 & 2,200 & 26,000 & 760 & $0.1^{*}$ & 169,000 \\
\hline $19.20 \%$ & $\$ 16$ & 55,000 & 120,000 & 10 & 47,000 & 37,000 & 5400 & $0.1^{*}$ & 162,000 \\
\hline $10.40 \%$ & $\$ 30$ & 63,000 & 150,000 & 15 & 3,200 & 27,000 & 3400 & 2.50 & 128,000 \\
\hline $8.70 \%$ & 50 & 57,000 & 170,000 & 18 & 1,900 & 26,000 & 2200 & 1.30 & 130,000 \\
\hline $7.30 \%$ & $\$ 100$ & 51,000 & 180,000 & 18 & 2,000 & 27,000 & 2300 & 6.00 & 123,000 \\
\hline $3.60 \%$ & $\$ 200$ & 47,000 & 200,000 & 26 & 1,300 & 21,000 & 2400 & 4.10 & 89,000 \\
\hline $2.10 \%$ & -200 & 41,000 & 250,000 & 42 & 1,200 & 15,000 & 5500 & 7.40 & 59,000 \\
\hline \multicolumn{2}{|c|}{ Ske fraction woighted avg. $=$} & 50,508 & 108,390 & 11 & 10,328 & 22,158 & 2.155 & 1.16 & 114,271 \\
\hline \multicolumn{10}{|c|}{\begin{tabular}{c|c}
$\boldsymbol{u}$ \\
\end{tabular}} \\
\hline & HFOWER Bottom & & & & & & & & \\
\hline $27.20 \%$ & 8 & 43,000 & 79,000 & 85 & 21,000 & 25,000 & 25000 & $0.1^{\circ}$ & 198,000 \\
\hline $25.60 \%$ & $\$ 16$ & 69,000 & 92,000 & $9^{\circ}$ & 1,000 & 21,000 & 920 & $0.1^{\circ}$ & 174,000 \\
\hline $10.30 \%$ & 130 & 65.000 & 12,000 & $1^{\circ}$ & 4,300 & 27,000 & 760 & $0.1^{\circ}$ & 157,000 \\
\hline $5.50 \%$ & 150 & 73,000 & 150,000 & 6 & 3,800 & 28,000 & 1800 & $0.1^{\circ}$ & 134,000 \\
\hline $3.30 \%$ & 100 & 73,000 & 160,000 & 4 & 5.200 & 25.000 & 2400 & 0.2 & 114,000 \\
\hline $1.60 \%$ & 200 & 71,000 & 150,000 & 16 & 3,600 & 16,000 & 2400 & $0.1^{\circ}$ & 105,000 \\
\hline $1.90 \%$ & -200 & 70,000 & 160,000 & 25 & 3,500 & 16,000 & 2100 & $0.1^{*}$ & 91,000 \\
\hline \multicolumn{2}{|c|}{ Size fraction woighted avg. $=$} & 44,945 & 65,246 & 25 & 6,916 & 17,882 & 7,370 & 0.08 & $\mathbf{1 2 9 . 1 1 2}$ \\
\hline \multicolumn{10}{|c|}{ 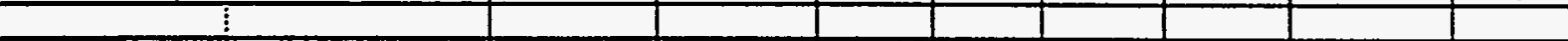 } \\
\hline & IWaipahu Combined & & & & & & & & \\
\hline $15.80 \%$ & $B$ & 86,000 & 37,000 & 18 & 600 & 58,000 & 2200 & $0.1^{\circ}$ & 186,000 \\
\hline $10.10 \%$ & $\$ 16$ & 62,000 & 69,000 & 4 & 570 & 99,000 & 500 & $0.1=$ & 163,000 \\
\hline $6.70 \%$ & 130 & 70.000 & 87.000 & 11 & 5,900 & 7,200 & 450 & $0.1^{\circ}$ & 138,000 \\
\hline $5.60 \%$ & 150 & 61,000 & 89,000 & 23 & 4,000 & 66,000 & 2200 & $0.1^{*}$ & 117,000 \\
\hline $5.20 \%$ & $\$ 100$ & 52,000 & 97,000 & 49 & 2,500 & 37,000 & 3100 & $0.1^{*}$ & 128,000 \\
\hline $7.10 \%$ & 200 & 51,000 & 130,000 & 110 & 2,400 & 30,000 & 5000 & 0.3 & 103,000 \\
\hline $1.10 \%$ & -200 & 50,000 & 120,000 & 180 & 1,200 & 25,000 & 4300 & 0.5 & 108,000 \\
\hline \multicolumn{2}{|c|}{ Ske fraction woighted avg.= } & 34,831 & 39.222 & 18 & 1,085 & 27,670 & 1,115 & 0.07 & 76.806 \\
\hline \multicolumn{2}{|c|}{ All ash type averages= } & 43,428 & 70,953 & 18 & 6.109 & 22,570 & 3,547 & 0.44 & 106,730 \\
\hline
\end{tabular}

Table 1 
Sodium through Density

Refer to Table 2 on page $\mathrm{B} 2-5$

Sodium - Average: 9,203 PPM. Like the iron analyses, there was little variability among the ash types or the ash size fractions. No sodium analyses were performed in the Task 1 mixtures.

Zinc - Average: 2,398 PPM. The averages for the H-POWER ash types were much lower than in the Task 1 analyses. The difference, however, is not considered significant. The consistency in the zinc concentrations found in the H-POWER combined ash was not evident in the Waipahu ash where a much wider variability was noted. There was also some enrichment in the smaller sizes of the H-POWER bottom ash. There is no explanation for the variability.

Sulfates - Average: 0.118 . The levels were higher in the Waipahu ash than in the H-POWER combined ash. This surprising result cannot be explained since one would expect the capture of $\mathrm{SO}_{2}$ by the $\mathrm{H}$-POWER scrubbers would result in much higher sulfate levels in that ash type. Perhaps the drying of the ash prior to sieving drove off the $\mathrm{SO}_{4}$.

Chlorides - Average: 0.808 . The chlorides were higher in the H-POWER combined ash than the bottom ash as expected, but in levels lower than expected. The concentrations were, however, consistent with what was detected in the Task 1 analysis. There was evidence of some enrichment of chlorides in the \#200 and smaller mesh sizes.

$\mathrm{pH}$ - The $\mathrm{pH}$ results were consistently in the range of about 11 - 12 for H-POWER combined and bottom ash. The Waipahu combined ash, lacking a lime scrubber system, averaged 10.7 .

Moisture - The moisture levels were affected by the drying required before performing the sieving. Thus analysis would not be worthwhile.

Density - The density levels are in the range of $2-3 \mathrm{~g} / \mathrm{cc}$ for all ash types and size fractions except for \#100 sieve fraction in the Waipahu combined ash which had a density of $11.3 \mathrm{~g} / \mathrm{Cc}$. 


\begin{tabular}{|c|c|c|c|c|c|c|c|c|c|}
\hline \multicolumn{10}{|c|}{ Chemical Analysis in units of PPM unless otherwise indicated } \\
\hline \% in size fraction & Ash Type/Sizo & $\mathrm{Na}$ & Zn & क SO4 & $\% \mathrm{Cl}_{2}$ & $\mathrm{pH}$ & \% moisture & Density, true & Density,app \\
\hline & HPOWER combined & & & & & & & g/ce & \\
\hline $26.70 \%$ & \begin{tabular}{|l|l|l}
8 \\
\end{tabular} & 12,000 & 2,400 & $\overline{0.03}$ & 2.01 & 11.60 & 0.26 & 2.57 & 0.92 \\
\hline $19.20 \%$ & $\$ 16$ & 15,000 & 3,400 & 0.03 & 1.51 & 11.70 & 0.00 & 2.36 & 0.81 \\
\hline $10.40 \%$ & $\mathbf{3 0}$ & 16,000 & 2,300 & 0.05 & 2.06 & 11.80 & 0.50 & 2.51 & 0.82 \\
\hline $8.70 \%$ & $\$ 50$ & 17,000 & 4,100 & 0.10 & 2.49 & 11.83 & 1.05 & 2.58 & 0.81 \\
\hline $7.30 \%$ & $\$ 100$ & 19,000 & 4,200 & 0.09 & 2.89 & 11.65 & 0.78 & 2.73 & 0.72 \\
\hline $3.60 \%$ & $\$ 200$ & 19,000 & 5,300 & 0.16 & 4.08 & 12.03 & 0.91 & 2.66 & 0.69 \\
\hline $2.10 \%$ & -200 & 22,000 & 9,200 & 0.61 & 9.48 & 11.88 & 1.54 & 3.17 & 0.56 \\
\hline \multicolumn{2}{|c|}{ Size fraction woighted avg. $=$} & 11,760 & 2,580 & 0.05 & 1.81 & 11.78 & 0.33 & 2.55 & 0.76 \\
\hline & & & & & & & & & \\
\hline & HPOWER Bottom & & & & & & & & \\
\hline $27.20 \%$ & $\$ 8$ & 10,000 & 490 & 0.00 & 0.06 & 11.11 & 0.06 & 2.59 & 0.98 \\
\hline $25.60 \%$ & $\$ 16$ & 15,000 & 420 & 0.38 & 0.09 & 11.42 & 0.02 & 2.49 & 0.85 \\
\hline $10.30 \%$ & $\$ 30$ & 16,000 & 980 & 0.02 & 0.18 & 11.73 & 0.90 & 2.45 & 0.85 \\
\hline $5.50 \%$ & $\$ 50$ & 17,000 & 4,300 & 0.04 & 0.25 & 11.75 & 1.26 & 2.16 & 0.74 \\
\hline $3.30 \%$ & $\$ 100$ & 16,000 & 5,500 & 0.16 & 0.42 & 11.53 & 1.96 & 2.97 & 0.93 \\
\hline $1.60 \%$ & $\$ 200$ & 16,000 & 5,500 & 0.31 & 0.58 & 11.31 & 3.64 & 4.85 & $0 . \overline{3}$ \\
\hline $1.90 \%$ & -200 & 15,000 & 5.200 & 0.31 & 0.74 & 11.48 & 2.88 & 1.04 & 0.60 \\
\hline \multicolumn{2}{|c|}{ Size fraction weightod aug. $=$} & 10,212 & 947 & 0.14 & 0.11 & 11.48 & 0.36 & 2.65 & 0.80 \\
\hline \multirow{2}{*}{\multicolumn{10}{|c|}{ Waipehu Combined }} \\
\hline & & & & & & & & & \\
\hline $15.80 \%$ & 18 & 7,700 & 700 & 0.12 & 0.19 & 10.69 & 0.00 & 2.73 & 0.91 \\
\hline $10.10 \%$ & 16 & 12,000 & 640 & 0.21 & 0.34 & 11.02 & 0.01 & 2.91 & 0.90 \\
\hline $6.70 \%$ & $\$ 30$ & 12,000 & 21,000 & 0.32 & 3.55 & 10.59 & 0.22 & 2.67 & 0.73 \\
\hline $5.60 \%$ & 450 & 12,000 & 7,000 & 0.41 & 0.65 & 10.64 & 0.40 & 2.37 & 0.59 \\
\hline $5.20 \%$ & 100 & 13,000 & 9,100 & 0.46 & 0.93 & 10.74 & 0.16 & 11.30 & 0.55 \\
\hline $7.10 \%$ & $\$ 200$ & 13,000 & 95,000 & 0.44 & 1.08 & 10.88 & 0.16 & 4.13 & 0.67 \\
\hline $1.10 \%$ & .200 & 12,000 & 14,000 & 0.85 & 0.88 & 10.50 & 2.18 & 2.25 & 0.80 \\
\hline \multicolumn{2}{|c|}{ Size fraction weighted avg.= } & 5,636 & 3,666 & 0.15 & 0.47 & 10.72 & 0.08 & 4.05 & 0.74 \\
\hline & All ash type avorages= & 9,203 & 2,398 & 0.11 & 0.80 & 11.33 & 0.26 & 3.12 & 0.76 \\
\hline \multicolumn{9}{|c|}{ Note: when results indicate non- detected, $1 / 2$ detection level used in place of 0.00} & \\
\hline
\end{tabular}

Table 2

\section{Conclusions}

Task 2 chemical results were on the whole as expected. The data. was relatively consistent with the Task 1 chemical data and showed no significant enrichment of trace metals in the smaller size fractions. The three ash types were also relatively consistent in their respective concentrations. One concern is the near $1 \%$ chloride content. This could possibly become a nuisance in situations where reuse may come in contact with steel rebar. 


\section{Laboratory Report}

Client: $\quad$ H-Power

91-174 Hanua Street

Kapolei, HI 96707

Attention: Mr. Denny Kort

\author{
Page: 1 of \\ ELP Project No.: $\quad 545$ \\ Report Date: $28-$ Feb-9
}

Sample Description: Samples from 91-174 Hanua St.

Sample Matrix: Screened Ash
Date Collected: 29-Dec- 9 Date Received: 29-Dec-s

\begin{tabular}{|c|c|c|c|c|c|c|c|c|}
\hline & & & $\begin{array}{r}\text { Client ID: } \\
\text { Matrix: } \\
\text { Lab ID: }\end{array}$ & & Method Blank & $\begin{array}{c}H M+8 \\
\text { ash } \\
122993.05\end{array}$ & $\begin{array}{c}\mathrm{HM}+16 \\
\text { ash } \\
122993-06 \\
\end{array}$ & $\begin{array}{c}\mathrm{HM}+30 \\
\text { ash } \\
122993-0\end{array}$ \\
\hline $\begin{array}{l}\text { Date } \\
07-F e b-94\end{array}$ & $\begin{array}{l}\text { Analysis } \\
\text { Metals Digestion..* }\end{array}$ & $\begin{array}{l}\text { Method } \\
\text { EPA 3050M }\end{array}$ & Units & MRL & Results & Results & Results & Results \\
\hline 28-Jan-94 & Metals Digestion & EPA 3050 & & & & & & \\
\hline 10-Feb-94 & Aluminum & EPA 6010 & $\mathrm{mg} / \mathrm{Kg}(\mathrm{ppm})$ & 20 & ND & 83,000 & 55,000 & 63,000 \\
\hline 10-Feb-94 & Cadmium & EPA 6010 & $\mathrm{mg} / \mathrm{Kg}(\mathrm{ppm})$ & 2 & ND & 9 & 10 & 15 \\
\hline 10-Feb-94 & Calcium & EPA 6010 & $\mathrm{mg} / \mathrm{Kg}(\mathrm{ppm})$ & 20 & ND & 110,000 & 120,000 & 150,006 \\
\hline 10-Feb-94 & Copper & EPA 6010 & $\mathrm{mg} / \mathrm{Kg}(\mathrm{ppm})$ & 10 & ND & 2,200 & 47,000 & 3,200 \\
\hline 10-Feb-94 & Iron & EPA 6010 & $\mathrm{mg} / \mathrm{Kg}$ (ppm) & 10 & ND & 26,000 & 37,000 & 27,000 \\
\hline 10-Feb-94 & Sodium & EPA 6010 & $\mathrm{mg} / \mathrm{Kg}(\mathrm{ppm})$ & 20 & ND & 12,000 & 15,000 & 16,000 \\
\hline 10-Feb-94 & Lead & EPA 6010 & $\mathrm{mg} / \mathrm{Kg}(\mathrm{ppm})$ & 20 & ND & 760 & 5,400 & 3,400 \\
\hline 10-Feb-94 & Zinc & EPA 6010 & $\mathrm{mg} / \mathrm{Kg}(\mathrm{ppm})$ & 10 & ND & 2,400 & 3,400 & 2,300 \\
\hline 03-Jan-94 & Mercury & EPA 7471 & $\mathrm{mg} / \mathrm{Kg}(\mathrm{ppm})$ & 0.2 & ND & ND & ND & 2.5 \\
\hline 11-Jan-94 & $\mathrm{pH}$ & EPA 9045 & units & 0.01 & NA & 11.60 & 11.70 & 11.80 \\
\hline 26-Jan-94 & Moisture & CLP ILM 2.0 & $\%$ & NA & ND & 0.26 & 0.00 & 0.50 \\
\hline 05-Jan-94 & Density, True ${ }^{* *}$ & & $g / c c$ & NA & NA & 2.57 & 2.36 & 2.51 \\
\hline 05-Jan-94 & Density (apparent) & & g/ce & 0.5 & NA & 0.922 & 0.810 & 0.816 \\
\hline 07-Jan-94 & Sulfate $\%$ & EPA 375.4 & $\%$ & $0.001^{\circ}$ & ND & 0.025 & 0.025 & 0.045 \\
\hline 07-Jan-94 & Chloride \% & EPA 325.3 & $\%$ & 0.001 & ND & 2.01 & 1.51 & 2.06 \\
\hline $16-F e b-94$ & Silicon* & EPA 6010 & $\mathrm{mg} / \mathrm{Kg}$ & 400 & ND & 169,000 & 162,000 & 128,001 \\
\hline
\end{tabular}

- Perkin-Elmer " Analytical Methods for AAS", Jan. 1982.

* Methods of Soil Ana' 'sis, American Society of Agronomy, Part 1, 1982.

** Lithium Metaborate Fusion digestion procedure performed by E. DeCarlo, Ph.D., University of Hawaii.
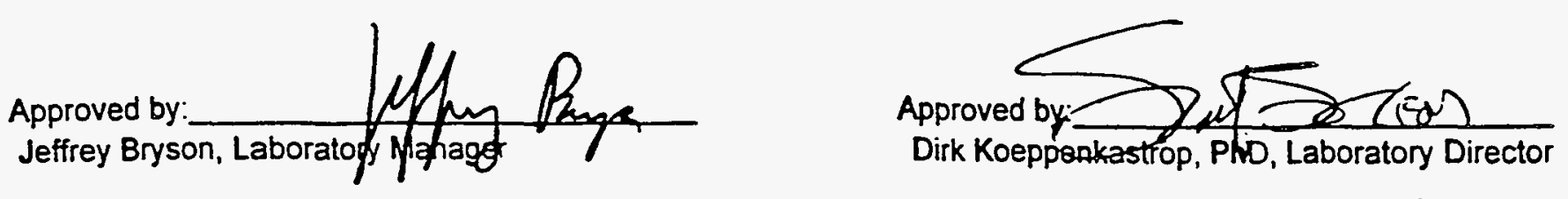
Page.

2 of 8

ELP Project No.:

5454

Report Date: 28-Feb-94

\begin{tabular}{|r|rcccc|}
\hline $\begin{array}{r}\text { Client ID: } \\
\text { Matrix: }\end{array}$ & $\begin{array}{c}H M+50 \\
\text { ash }\end{array}$ & $\begin{array}{c}H M+100 \\
\text { ash }\end{array}$ & $\begin{array}{c}H M+200 \\
\text { ash }\end{array}$ & $\begin{array}{c}H M-200 \\
\text { ash }\end{array}$ \\
\hline
\end{tabular}

2ate Analysis

17-Feb-94

!8-Jan-94

10-Feb-94

10-Feb-94

10-Feb-94

10-Feb-94

10-Feb-94

10-Feb-94

10-Feb-94

10-Feb-94

כ3-Jan-94

11-Jan-94

26-Jan-94

05-Jan-94

05-Jan-94

07-Jan-94

07-Jan-94

16-Feb-94
Metals Digestion ***

Metals Digestion

Aluminum

Cadmium

Calcium

Copper

Iron

Sodium

Lead

Zinc

Mercury

$\mathrm{pH}$

Moisture

Density, True**

Densily (apparent) ${ }^{* *}$

Sulfate \%

Chloride \%

Silicon"

$\begin{array}{lcc}\text { Method } & \text { Units } & \text { MRL } \\ \text { EPA 3050M } & & \\ \text { EPA 3050 } & & \\ \text { EPA 6010 } & \mathrm{mg} / \mathrm{Kg}(\mathrm{ppm}) & 20 \\ \text { EPA 6010 } & \mathrm{mg} / \mathrm{Kg}(\mathrm{ppm}) & 2 \\ \text { EPA 6010 } & \mathrm{mg} / \mathrm{Kg}(\mathrm{ppm}) & 20 \\ \text { EPA 6010 } & \mathrm{mg} / \mathrm{Kg}(\mathrm{ppm}) & 10 \\ \text { EPA 6010 } & \mathrm{mg} / \mathrm{Kg}(\mathrm{ppm}) & 10 \\ \text { EPA 6010 } & \mathrm{mg} / \mathrm{Kg}(\mathrm{ppm}) & 20 \\ \text { EPA 6010 } & \mathrm{mg} / \mathrm{Kg}(\mathrm{ppm}) & 20 \\ \text { EPA 6010 } & \mathrm{mg} / \mathrm{Kg}(\mathrm{ppm}) & 10 \\ \text { EPA 7471 } & \mathrm{mg} / \mathrm{Kg}(\mathrm{ppm}) & 0.2 \\ \text { EPA 9045 } & \text { units } & 0.01 \\ \text { CLP ILM 2.0 } & \% & \mathrm{NA} \\ & \mathrm{g} / \mathrm{cc} & \mathrm{NA} \\ & \mathrm{g} / \mathrm{cc} & 0.5 \\ \text { EPA 375.4 } & \% & 0.001 \\ \text { EPA 325.3 } & \% & 0.001 \\ \text { EPA 6010 } & \mathrm{mg} / \mathrm{Kg} & 400\end{array}$

Results

Results

Results

Results Results

$\begin{array}{ccccc}\text { ND } & 57,000 & 51,000 & 47,000 & 41,000 \\ \text { ND } & 18 & 18 & 26 & 42 \\ \text { ND } & 170,000 & 180,000 & 200,000 & 250,000 \\ \text { ND } & 1,900 & 2,000 & 1,300 & 1,200 \\ \text { ND } & 26,000 & 27,000 & 21,000 & 15,000 \\ \text { ND } & 17,000 & 19,000 & 19,000 & 22,000 \\ \text { ND } & 2,200 & 2,300 & 2,400 & 5,500 \\ \text { ND } & 4,100 & 4,200 & \mathbf{5 , 3 0 0} & 9,200 \\ \text { ND } & 1.3 & 6.0 & 4.1 & 7.4 \\ \text { NA } & 11.83 & 11.65 & 12.03 & 11.88 \\ \text { ND } & 1.05 & 0.78 & 0.91 & 1.54 \\ \text { NA } & 2.58 & 2.73 & 2.66 & 3.17 \\ \text { NA } & 0.813 & 0.715 & 0.685 & 0.560 \\ \text { ND } & 0.096 & 0.090 & 0.160 & 0.610 \\ \text { ND } & 2.49 & 2.89 & 4.08 & 9.48 \\ \text { ND } & 130,000 & 123,000 & 89,000 & 59,000\end{array}$

- Perkin-Elmer " Analytical Methods for AAS", Jan. 1982.

* Methods of Soil Analysis, American Society of Agronomy, Part 1, 1982.

*** Lithium Metaborate Fusion digestion procedure performed by E. DeCarlo, Ph.D., University of Hawaii.
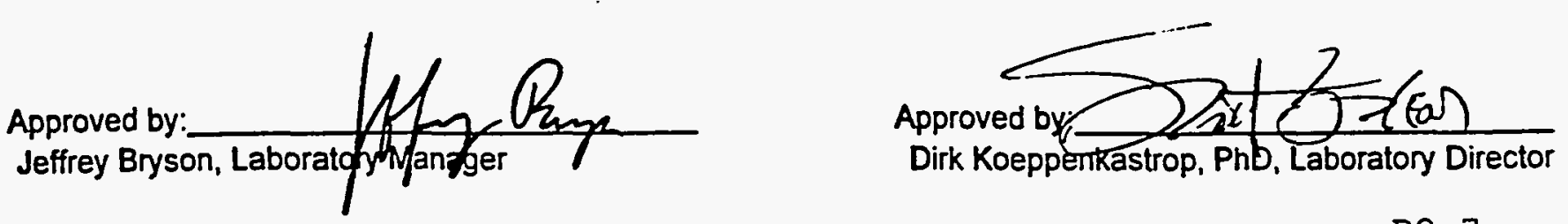
Page: $\quad 3$ of

ELP Project No.: $\quad 545$

Report Date. 28-Feb-؟

\begin{tabular}{|r|rccc}
\hline Client ID: & $\mathrm{HB}+8$ & $\mathrm{HB}+16$ & $\mathrm{HB}+30$ & $\mathrm{HB}+50$ \\
Matrix: & ash & ash & ash & ash \\
Lab ID: & Method Blan 122993-12 & $122993-13$ & $122993-14$ & $122993-1$ \\
\hline
\end{tabular}

\begin{tabular}{|c|c|c|c|c|c|c|c|c|c|}
\hline $\begin{array}{l}\text { Date } \\
\text { 07-Feb-94 }\end{array}$ & $\begin{array}{l}\text { Analysis } \\
\text { Metals Digestion }\end{array}$ & $\begin{array}{l}\text { Method } \\
\text { EPA 3050M }\end{array}$ & Units & MRL & Results & Results & Results & Results & Results \\
\hline 28-Jan-94 & Metals Digestion & EPA 3050 & & & & & & & \\
\hline 10-Feb-94 & Aluminum & EPA 6040 & $\mathrm{mg} / \mathrm{Kg}(\mathrm{ppm})$ & 20 & ND & 43,000 & 69,000 & 65,000 & 73,000 \\
\hline 10-Feb-94 & Cadmium & EPA 6010 & $\mathrm{mg} / \mathrm{Kg}(\mathrm{ppm})$ & 2 & ND & 85 & ND & ND & 6 \\
\hline 10-Feb-94 & Calcium & EPA 6010 & $\mathrm{mg} / \mathrm{Kg}(\mathrm{ppm})$ & 20 & ND & 79,000 & 92,000 & 12,000 & 150,000 \\
\hline 10-Feb-94 & Copper & EPA 6010 & $\mathrm{mg} / \mathrm{Kg}(\mathrm{ppm})$ & 10 & ND & 21,000 & 1,000 & 4,300 & 3,800 \\
\hline 10-Feb-94 & Iron & EPA 6010 & $\mathrm{mg} / \mathrm{Kg}(\mathrm{ppm})$ & 10 & ND & 25,000 & 21,000 & 27,000 & 28,000 \\
\hline 10-Feb-94 & Sodium & EPA 6010 & $\mathrm{mg} / \mathrm{Kg}(\mathrm{ppm})$ & 20 & ND & 10,000 & 15,000 & 16,000 & 17,000 \\
\hline 10-Feb-94 & Lead & EPA 6010 & $\mathrm{mg} / \mathrm{Kg}(\mathrm{ppm})$ & 20 & ND & 25,000 & 920 & 760 & 1,800 \\
\hline 10-Feb-94 & Zinc & EPA 6010 & $\mathrm{mg} / \mathrm{Kg}(\mathrm{ppm})$ & 10 & ND & 490 & 420 & 980 & 4,300 \\
\hline 03-Jan-94 & Mercury & EPA 7471 & mg/Kg (ppm) & 0.2 & ND & ND & ND & ND & ND \\
\hline 11-Jan-94 & $\mathrm{pH}$ & EPA 9045 & units & 0.01 & NA & 11.11 & 11.42 & 11.73 & 11.75 \\
\hline 26-Jan-94 & Moisture & CLP ILM 2. & $\%$ & NA & ND & 0.06 & 0.02 & 0.90 & 1.26 \\
\hline 05-Jan-94 & Density, True ${ }^{* *}$ & & $g / c c$ & NA & NA & 2.59 & 2.49 & 2.45 & 2.16 \\
\hline 05-Jan-94 & Density (apparent) & 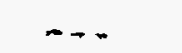 & $g / c c$ & 0.5 & NA & 0.981 & 0.847 & 0.846 & 0.736 \\
\hline 07-Jan-94 & Sulfate $\%$ & EPA 375.4 & $\%$ & 0.001 & ND & 0.087 & 0.380 & 0.017 & 0.042 \\
\hline 07-Jan-94 & Chloride \% & EPA 325.3 & $\%$ & 0.001 & ND & 0.055 & 0.091 & 0.18 & 0.25 \\
\hline 16-Feb-94 & Silicon & EPA 6010 & $\mathrm{mg} / \mathrm{Kg}$ & 400 & ND & 198,000 & 174,000 & 157,000 & 134,001 \\
\hline
\end{tabular}

- Perkin-Elmer " Analytical Methods for AAS", Jan. 1982.

** Methods of Soil Analysis, American Society c\& Agronomy, Part 1, 1982.

** Lithium Metaborate Fusion digestion procedure performed by E. DeCarlo, Ph.D., University of Hawaii.
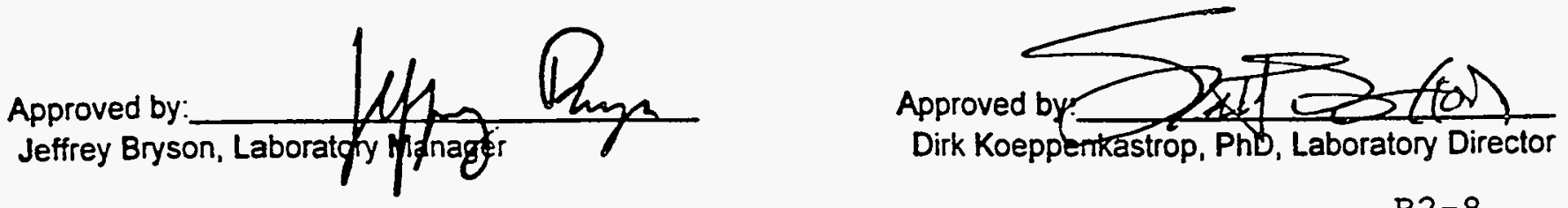
Page: $\quad 4$ of 8

ELP Project No:: $\quad 5454$

Report Date: 28-Feb-94

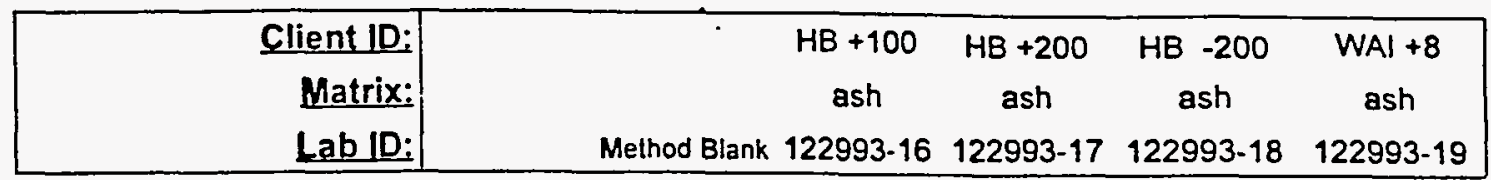

\begin{tabular}{|c|c|c|c|c|c|c|c|c|c|}
\hline Date & Analysis & Method & Units & MRL & Results & Results & Ressults & Results & Results \\
\hline 07-Feb-94 & Metals Digestion"** & EPA $3050 \mathrm{M}$ & & & & & & & \\
\hline 28-Jan-94 & Metals Digestion & EPA 3050 & & & & & & & \\
\hline 10-Feb-94 & Aluminum & EPA 6010 & $\mathrm{mg} / \mathrm{Kg}(\mathrm{ppm})$ & 20 & ND & 73,000 & 71,000 & 70,000 & 86,000 \\
\hline 10-Feb-94 & Cadmium & EPA 6010 & $\mathrm{mg} / \mathrm{Kg}(\mathrm{ppm})$ & 2 & ND & 4 & 16 & 25 & 18 \\
\hline 10-Feb-94 & Calcium & EPA 6010 & $\mathrm{mg} / \mathrm{Kg}(\mathrm{ppm})$ & 20 & ND & 160,000 & 150,000 & 160,000 & 37,000 \\
\hline 10-Feb-94 & Copper & EPA 6010 & $\mathrm{mg} / \mathrm{Kg}(\mathrm{ppm})$ & 10 & ND & 5,200 & 3,600 & 3,500 & 600 \\
\hline 10-Feb-94 & Iron & EPA 6010 & $\mathrm{mg} / \mathrm{Kg}(\mathrm{ppm})$ & 10 & ND & 25,000 & 16,000 & 16,000 & 58,000 \\
\hline 10-Feb-94 & Sodium & EPA 6010 & $\mathrm{mg} / \mathrm{Kg}(\mathrm{ppm})$ & 20 & ND & 16,000 & 16,000 & 15,000 & 7,700 \\
\hline 10-Feb-94 & Lead & EPA 6010 & $\mathrm{mg} / \mathrm{Kg}(\mathrm{ppm})$ & 20 & ND & 2,400 & 2,400 & 2,100 & 2,200 \\
\hline 10-Feb-94 & Zinc & EPA 6010 & $\mathrm{mg} / \mathrm{Kg}(\mathrm{ppm})$ & 10 & ND & 5,500 & 5,500 & 5,200 & 700 \\
\hline 03-Jan-94 & Mercury & EPA 7471 & $\mathrm{mg} / \mathrm{Kg}(\mathrm{ppm})$ & 0.2 & ND & 0.2 & ND & ND & ND \\
\hline 11-Jan-94 & $\mathrm{pH}$ & EPA 9045 & units & 0.01 & NA & 11.53 & 11.31 & 11.48 & 10.69 \\
\hline 26-Jan-94 & Moisture & CLP ILM 2.0 & $\%$ & NA & ND & 1.96 & 3.64 & 2.88 & 0.00 \\
\hline 05-Jan-94 & Density, True"* & & $g / c c$ & NA & NA & 2.97 & 4.85 & 1.04 & 2.73 \\
\hline 05-Jan-94 & Density (apparent) & & $g / c c$ & 0.5 & NA & 0.927 & 0.630 & 0.599 & 0.908 \\
\hline 07-Jan-94 & Sulfate $\%$ & EPA 375.4 & $\%$ & 0.001 & ND & 0.16 & 0.31 & 0.31 & 0.12 \\
\hline $07-J a n-94$ & Chloride \% & EPA 325.3 & $\%$ & 0.001 & ND & 0.42 & 0.58 & 0.74 & 0.19 \\
\hline 16-Feb-94 & Silicon* & EPA 6010 & $\mathrm{mg} / \mathrm{Kg}$ & 400 & ND & 114,000 & 105,000 & 91,000 & 186,000 \\
\hline
\end{tabular}

- Perkin-Elmer " Analytical Methods for AAS", Jan. 1982.

* Methods of Soil Analysis, American Society of Agronomy, Part 1, 1982.

*** Lithium Metaborate Fusion digestion procedure performed by E. DeCarlo, Ph.D., University of Hawaii.
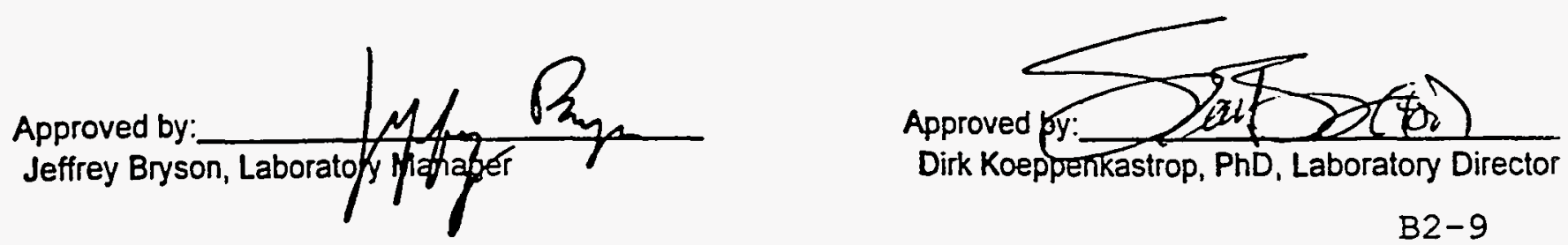

B2-9 
Report Date: 28-Feb-

\begin{tabular}{|r|rccc}
\hline $\begin{array}{r}\text { Client ID: } \\
\text { Matrix: }\end{array}$ & WAI +16 & WAI +30 & WAI +50 & WAI +1C \\
Lab ID: & ash & ash & ash & ash \\
\hline
\end{tabular}

\begin{tabular}{|c|c|c|c|c|c|c|c|c|c|}
\hline $\begin{array}{l}\text { Date } \\
07-F e b-94\end{array}$ & $\begin{array}{l}\text { Analysis } \\
\text { Metals Digestion... }\end{array}$ & $\begin{array}{l}\text { Method } \\
\text { EPA } 3050 M\end{array}$ & Units & MRL & Results & Besults & Resuits & Results & Result \\
\hline 28-Jan-94 & Metals Digestion & EPA 3050 & & & & & & & \\
\hline 10-Feb-94 & Aluminum & EPA 6010 & $\mathrm{mg} / \mathrm{Kg}(\mathrm{ppm})$ & 20 & ND & 62,000 & 70,000 & 61,000 & $52,00 C$ \\
\hline 10-Feb-94 & Cadmium & EPA 6010 & $\mathrm{mg} / \mathrm{Kg}(\mathrm{ppm})$ & 2 & ND & 4 & 11 & 23 & 49 \\
\hline 10-Feb-94 & Calcium & EPA 6010 & $\mathrm{mg} / \mathrm{Kg}(\mathrm{ppm})$ & 20 & ND & 69,000 & 87,000 & 89,000 & $97,00 C$ \\
\hline 10-Feb-94 & Copper & EPA 6010 & $\mathrm{mg} / \mathrm{Kg}(\mathrm{ppm})$ & 10 & ND & 570 & 5,900 & 4,000 & 2,500 \\
\hline 10-Feb-94 & Iron & EPA 6010 & $\mathrm{mg} / \mathrm{Kg}(\mathrm{ppm})$ & 10 & ND & 99,000 & 7,200 & 66,000 & $37,00 C$ \\
\hline 10-Feb-94 & Sodium & EPA 6010 & $\mathrm{mg} / \mathrm{Kg}(\mathrm{ppm})$ & 20 & ND & 12,000 & 12,000 & 12,000 & $13,00 C$ \\
\hline 10-Feb-94 & Lead & EPA 6010 & $\mathrm{mg} / \mathrm{Kg}(\mathrm{ppm})$ & 20 & ND & 500 & 450 & 2,200 & 3,100 \\
\hline 10-Feb-94 & Zinc & EPA 6010 & $\mathrm{mg} / \mathrm{Kg}(\mathrm{ppm})$ & 10 & ND & 640 & 21,000 & 7,000 & 9,100 \\
\hline 03-Jan-94 & Mercury & EPA 7471 & $\mathrm{mg} / \mathrm{Kg}(\mathrm{ppm})$ & 0.2 & ND & ND & ND & ND & ND \\
\hline 11-Jan-94 & $\mathrm{pH}$ & EPA 9045 & units & 0.01 & NA & 11.02 & 10.59 & 10.64 & 10.74 \\
\hline 26-Jan-94 & Moisture & CLP ILM 2.0 & $\%$ & NA & ND & 0.01 & 0.22 & 0.40 & 0.16 \\
\hline 05-Jan-94 & Density, True"* & & $g / c c$ & NA & NA & 2.91 & 2.67 & 2.37 & 14.30 \\
\hline 05-Jan-94 & Density (apparent) ${ }^{*}$ & & $g / c c$ & 0.5 & NA & 0.900 & 0.725 & 0.593 & 0.549 \\
\hline 07-Jan-94 & Sulfate $\%$ & EPA 375.4 & $\%$ & 0.001 & ND & 0.21 & 0.32 & 0.41 & 0.46 \\
\hline 07-Jan-94 & Chloride \% & EPA 325.3 & $\%$ & 0.001 & ND & 0.34 & 3.55 & 0.65 & 0.93 \\
\hline $16-\mathrm{Feb}-94$ & Silicon* & EPA 6010 & $\mathrm{mg} / \mathrm{Kg}$ & 400 & ND & 163,000 & 138,000 & 117,000 & 128,00 \\
\hline
\end{tabular}

"Perkin-Elmer " Analytical Methods for AAS", Jan. 1982.

* Methods of Soil Analysis, American Society of Agronomy, Part 1, 1982.

** Lithium Metaborate Fusion digestion procedure performed by E. DeCarlo, Ph.D., University of Hawaii.
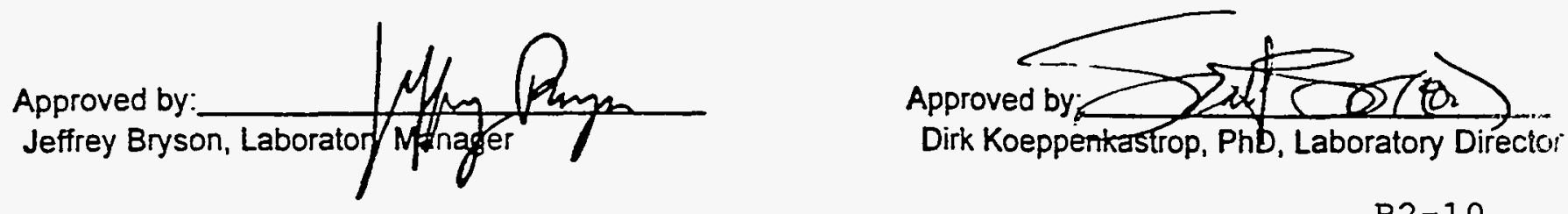
Page: $\quad 6$ of 8

ELP Project No.: $\quad 5454$

Report Date: 28-Feb-94

\begin{tabular}{|r|rcc|}
\hline $\begin{array}{r}\text { Client ID: } \\
\text { Matrix: } \\
\text { Lab 1D: }\end{array}$ & & WAI +200 & WAI -200 \\
\hline
\end{tabular}

\begin{tabular}{|c|c|c|c|c|c|c|c|}
\hline $\begin{array}{l}\text { Date } \\
\text { 07-Feb-94 }\end{array}$ & $\begin{array}{l}\text { Analysis } \\
\text { Metals Digestion*** }\end{array}$ & $\begin{array}{l}\text { Method } \\
\text { EPA 3050M }\end{array}$ & Units & MRL & Results & Resuits & Results \\
\hline 28-Jan-94 & Metals Digestion & EPA 3050 & & & & & \\
\hline 10-Feb-94 & Aluminum & EPA 6010 & $\mathrm{mg} / \mathrm{Kg}(\mathrm{ppm})$ & 20 & ND & 51,000 & 50,000 \\
\hline 10-Feb-94 & Cadmium & EPA 6010 & $\mathrm{mg} / \mathrm{Kg}(\mathrm{ppm})$ & 2 & ND & 110 & 180 \\
\hline $10-F e b-94$ & Calcium & EPA 6010 & $\mathrm{mg} / \mathrm{Kg}(\mathrm{ppm})$ & 20 & ND & 130,000 & 120,000 \\
\hline 10-Feb-94 & Copper & EPA 6010 & $\mathrm{mg} / \mathrm{Kg}(\mathrm{ppm})$ & 10 & ND & 2,400 & 1,200 \\
\hline 10-Feb-94 & Iron & EPA 6010 & $\mathrm{mg} / \mathrm{Kg}(\mathrm{ppm})$ & 10 & ND & 30,000 & 25,000 \\
\hline 10-Feb-94 & Sodium & EPA 6010 & $\mathrm{mg} / \mathrm{Kg}(\mathrm{ppm})$ & 20 & ND & 13,000 & 12,000 \\
\hline 10-Feb-94 & Lead & EPA 6010 & $\mathrm{mg} / \mathrm{Kg}(\mathrm{ppm})$ & 20 & ND & 5,000 & 4,300 \\
\hline 10-Feb-94 & Zinc & EPA 6010 & $\mathrm{mg} / \mathrm{Kg}(\mathrm{ppm})$ & 10 & ND & 15,000 & 14,000 \\
\hline 03-Jan-94 & Mercury & EPA 7471 & $\mathrm{mg} / \mathrm{Kg}(\mathrm{ppm})$ & 0.2 & ND & 0.3 & 0.5 \\
\hline 11-Jan-94 & $\mathrm{pH}$ & EPA 9045 & units & 0.01 & NA & 10.88 & 10.50 \\
\hline 26-Jan-94 & Moisture & CLP ILM 2.0 & $\%$ & NA & ND & 0.16 & 2.18 \\
\hline 05-Jan-94 & Density, True** & & $g / c c$ & NA & NA & 4.13 & 2.25 \\
\hline 05-Jan-94 & Density (apparent)"* & & $g / c c$ & 0.5 & NA & 0.466 & 0.798 \\
\hline $07-J a n-94$ & Sulfate \% & EPA 375.4 & $\%$ & 0.001 & ND & 0.44 & 0.85 \\
\hline 07-Jan-94 & Chloride \% & EPA 325.3 & $\%$ & 0.001 & ND & 1.08 & 0.88 \\
\hline 16-Feb-94 & Silicon* & EPA 6010 & $\mathrm{mg} / \mathrm{Kg}$ & 400 & ND & 103,000 & 108,000 \\
\hline
\end{tabular}

- Perkin-Elmer "Analytical Methods for AAS", Jan. 1982.

** Methods of Soil Analysis, American Society of Agronomy, Part 1, 1982.

*.* Lithium Metaborate Fusion digestion procedure performed by E. DeCarlo, Ph.D., University of Hawaii.
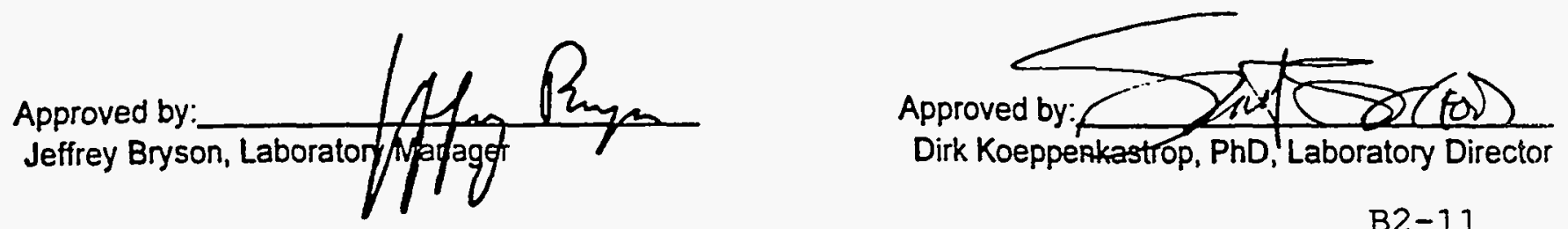

B2-11 


\section{Quality Control Data}

\begin{tabular}{|c|c|c|c|c|c|c|c|c|}
\hline \multirow{2}{*}{$\begin{array}{l}\text { SPIKES } \\
\text { Lab ID }\end{array}$} & & $\begin{array}{l}\text { Lab ID: } \\
\text { Units: }\end{array}$ & $\begin{array}{c}\text { LCS1 } \\
\% R\end{array}$ & $\begin{array}{c}\text { LCS2 } \\
\% R\end{array}$ & RPD & $\begin{array}{l}\text { MS } \\
\% R \\
\end{array}$ & $\begin{array}{c}\text { MSD } \\
\% R\end{array}$ & RPD \\
\hline & Analysis & Method & Results & Results & Results & Results & Results & Results \\
\hline $122993-55$ & Aluminum & EPA 6010 & 94 & 94 & 0 & $* * * *$ & $\neq * * *$ & **** \\
\hline $122993-05$ & Cadmium & EPA 6010 & 103 & 109 & 6 & $* * * *$ & $* * * *$ & $* * * *$ \\
\hline 122993-05 & Cadmium & EPA 6010 & NA & NA & NA & 79 & 77 & 3 \\
\hline 122993-05 & Cadmium & EPA 6010 & 88 & 88 & 0 & 79 & 77 & 3 \\
\hline $122993-25$ & Calcium & EPA 6010 & 83 & 81 & 2 & $* * * *$ & $\star * * *$ & $* * * *$ \\
\hline $122993-25$ & Calcium & EPA 6010 & 117 & 117 & 0 & $* * * *$ & $* * * *$ & $* * * *$ \\
\hline $122993-25$ & Copper & EPA 6010 & 94 & 97 & 3 & $* * * *$ & **** & $* * \star *$ \\
\hline $122993-25$ & Copper & EPA 6010 & 103 & 106 & 3 & $* * *$ & $* * * *$ & $* * * *$ \\
\hline $122993-25$ & Iron & EPA 6010 & 101 & 102 & 1 & $* * * * *$ & ...* & $* * * *$ \\
\hline $122993-25$ & Iron & EPA 6010 & 106 & 108 & 2 & $\ldots * *$ & $* * * *$ & $* * * *$ \\
\hline $122993-25$ & Sodium & EPA 6010 & 93 & 90 & 3 & m.* & $* * * *$ & $* \cdots *$ \\
\hline $122993-25$ & Sodium & EPA 6010 & 99 & 105 & 6 & ***** & ***** & $\cdots * * *$ \\
\hline $122993-25$ & Lead & EPA 6010 & 101 & 103 & 2 & **** & $* * * *$ & $* * * *$ \\
\hline $122993-25$ & Lead & EPA 6010 & 104 & 108 & 4 & $* * * *$ & **** & $\cdots * *$ \\
\hline $122993-25$ & Zinc & EPA 6010 & 97 & 97 & 0 & **** & **** & ***** \\
\hline $122993-25$ & Zinc & EPA 6010 & 103 & 104 & 1 & **** & $\boldsymbol{t * * *}$ & 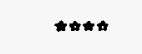 \\
\hline $122993-20$ & Mercury & EPA 7471 & 99 & 99 & 0 & 121 & 115 & 5 \\
\hline $122993-20$ & Mercury & EPA 7471 & 116 & 108 & 7 & 109 & 100 & 9 \\
\hline 012294-08 & Sulfate \% & EPA 375.4 & 115 & 113 & 2 & 108 & 104 & 4 \\
\hline $122993-18$ & Sulfate \% & EPA 375.4 & 94 & 97 & 3 & 102 & 101 & 1 \\
\hline $122993-25$ & Chloride \% & EPA 325.3 & 99 & 99 & 0 & 101 & 99 & 2 \\
\hline $122993-10$ & Chloride \% & EPA 325.3 & 100 & 98 & 2 & 99 & 100 & 1 \\
\hline
\end{tabular}

\begin{tabular}{lll}
\hline DUPLICATES & $\begin{array}{l}\text { Lab I } \\
\text { Unit }\end{array}$ \\
\hline Lab ID & Analysis & Method \\
& & \\
$122993-05$ & $\mathrm{pH}$ & EPA 9045 \\
$011094-01$ & $\mathrm{pH}$ & EPA 9045 \\
$122993-24$ & Density (true) & g/cc \\
$122993-24$ & Density (apparent) & $\mathrm{g} / \mathrm{cc}$ \\
$122993-08$ & Density (true) & g/cc \\
$122993-08$ & Density (apparent) & g/cc
\end{tabular}

OS

D

RPD percent

nits: are $\mathrm{mg} / \mathrm{L}$ unless otherwise noted

Results 


$\begin{array}{rr}\text { Page: } & 8 \text { of } 8 \\ \text { ELP Project No.: } & 5454 \\ \text { Report Date: } & 28-F e b-94\end{array}$

\section{Definitions}

$\begin{array}{ll}\text { D } & \text { Duplicate } \\ \text { LCS } & \text { Laboratory Control Sample } \\ \text { MS } & \text { Matrix Spike } \\ \text { MSD } & \text { Matrix Spike Duplicate } \\ \text { MRL } & \text { Method Reporting Limit } \\ \text { NA } & \text { Not Applicable } \\ \text { ND } & \text { Not Detected at the MRL } \\ \text { NR } & \text { Not Requested } \\ \text { OS } & \text { Original Sample } \\ \text { \%R } & \text { Percent Recovery } \\ \text { PDS } & \text { Post Digestion Spike } \\ \text { RPD } & \text { Relative Percent Difference }\end{array}$

Approved by:
Jeffrey Bryson, Laborato $y$ Magragit

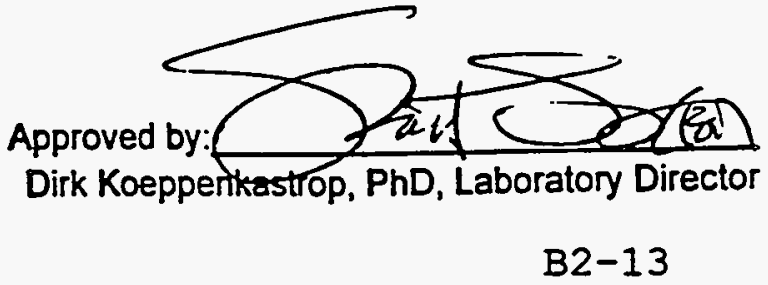




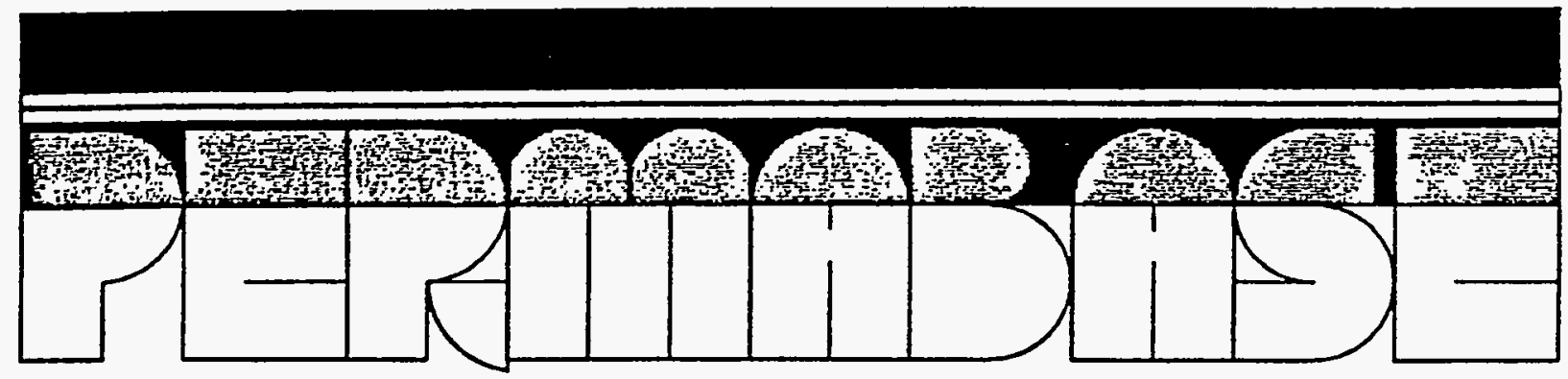

HONOLULU, HAWAII

REFUSE DERIVED FUEL COMBUSTOR ASH

FOR USE IN PERMABASE-PLUS

PRELIMINARY INVESTIGATION 


\author{
HONOLULU, HAWAII \\ REFUSE DERIVED FUEL COMBUSTOR ASH \\ FOR USE IN PERMABASE-PLUS \\ PRELIMINARY INVESTIGATION
}

One five gallon bucket of RDF bottom ash was received September 28, 1993, by Permabase personnel for evaluation as a potential aggregate in the proprietary soil cement material PERMABASE-PLUS. Review of the State of Hawaii, Department of Transportation Standard Specifications for Road and Bridge Construction Section 308 - Porland Cement Treated Base revealed that cement treated base is designed in Hawaii by controlling the quality of the ingredients and adding 5\% cement. This was confirmed in conversation with Walter Quroiwua of the HDOT Quality Control laboratory, Honolulu. The tests used to control the quality of the cement treated base ingredients are:
Los Angeles Abrasion
AASHTO T 96
Sand Equivalent
AASHTO T 176
Plasticity Index
AASHTO T 90
Flat or Elongated pieces
HWY-TC 4
Grading
AASHTO T 27

Combustor ash represents approximately $25 \%$ of the aggregate in PERMABASE-PLUS. Since the other $75 \%$ of the aggregate is composed of "local" materials we did not conduct the above five tests on our designs. However, through proper aggregate selection and combination we are confident that PERMABASE-PLUS made in part with Honolulu's RDF combustor ash can meet the appropriate quality requirements.

Our preliminary evaluation was based on Florida Department of Transportation design criteria in order to provide a correlation between the ash from Honolulu and previously tested ash. Tests were also conducted using a crushed concrete aggregate with the ash meeting the grading requirements specified in the HDOT manual. We chose crushed concrete due to the products universal availability and the potential for reuse. 


\section{Material Evaluation}

Bottom Ash: As delivered, the bottom ash was all minus $11 / 2$ inch material. Visual observation revealed a relatively large amount of glass/ceramic shards and a nugget appearance to the ash. Ferrous metals appeared to consist largely of wires, nails and screws: Sieve analysis and dry weight evaluation determined that $67 \%$ of the bottom ash was retained on the $\# 4(4.75 \mathrm{~mm})$ sieve which needs to be removed or reduced in size before use. The sieve analysis also showed that $2.1 \%$ of the material was smaller than a $\# 200$ (75 micron) sieve and would be considered fly ash.

Of the material retained on the $\# 4(4.75 \mathrm{~mm})$ sieve, $22 \%$ remained after the crushing process. This material consisted of ferrous, aluminum, other metals and paper/plastic/organics. Noncrushable material as a percentage of total dry weight of ash is as follows:

$\begin{array}{ll}\text { Ferrous: } & 6.6 \% \\ \text { Aluminum: } & 5.0 \% \\ \text { Misc Metal: } & 2.9 \% \\ \text { Paper, etc: } & 0.1 \%\end{array}$

Crushed Concrete Aggregate: A sample of this aggregate was obtained by Permabase personnel from a crushed concrete recycling operation in the Tampa Bay area. The aggregate was then size separated in the laboratory in the following manner: $+1 / 2^{n}$ material removed; $-1 / 2^{n} /+\# 4$ material separated from - \#4 material. PERMABASE-PLUS aggregate was then proportioned utilizing the processed Honolulu ash and the two sized fractions of the crushed concrete to conform to the HDOT grading specification.

Leisey Sand: This material consists of a fine graded silica sand, minus $\# 30$ (600 micron) sieve, plus $\$ 200$ (75 micron) sieve. This sand was used as a standard of comparison with other previously designed ash/sand soil cement products.

\section{PERMABASE-PLUS}

Two mixtures of PERMABASE-PLUS were created in the laboratory for comparative study. Mixture One consisted of 3 parts Leisey sand and 1 part processed bottom ash. Mixture Two consisted of 3 parts crushed concrete and 1 part processed bottom ash to meet the HDOT grading requirement. Both mixtures were proportioned with $5 \%$ cement prior to compaction of test specimens with varying moisture contents in accordance with AASHTO T 134. These specimens were then held for seven days in a moisture curing tank before unconfined compressive strength testing. 


\section{Comparison of Ash Products}

The Florida approved methods for evaluating soil cement are based on seven day unconfined compressive strength data. Previous ash investigations at ten combustors located throughout the United Sates have provided unconfined compressive strength data at $5 \%$ cement content from 290 pounds per square inch (psi) to $610 \mathrm{psi}$. Unit weights from these same materials range from 91 pounds per cubic foot (pcf) to $108 \mathrm{pcf}$.

Analysis performed on the Honolulu materials reveals that the unit weight of the Leisey sand/ash mixture at approximately $106 \mathrm{pcf}$ fits well within the range of other sand/ash mixtures tested to date. When crushed concrete is utilized in the mix, the unit weight increased to $111 \mathrm{pcf}$.

Compressive strength data generated by the seven day specimens revealed that the Leisey sand/ash results at $314 \mathrm{psi}$ were within the range of results generated by other Leisey sand/ash PERMABASE-PLUS products. The seven day strength for the crushed concrete/ash specimens at 661 psi is well above the typical strengths achieved with sand mixtures.

\section{Conclusion}

The purpose for this preliminary investigation was to determine the suitability of the materials supplied for the use as a soil cement base product based on its ability to withstand a load. Our evaluation reveals that the composite materials, as represented by our specimens, will make an excellent soil cement base product if properly prepared.

Further evaluation will be necessary to determine optimum material characteristics and to make recommendations based on local Hawaiian aggregates and design specifications. Further investigation will also be necessary to determine the suitability of Honolulu combined ash for an aggregate in PERMABASE-PLUS. Laboratory analyses are included for your review on the attached sheets. 


\section{PERMABASE-PLUS}

Honolulu RDF Ash/Crushed Concrete

Sieve Analysis
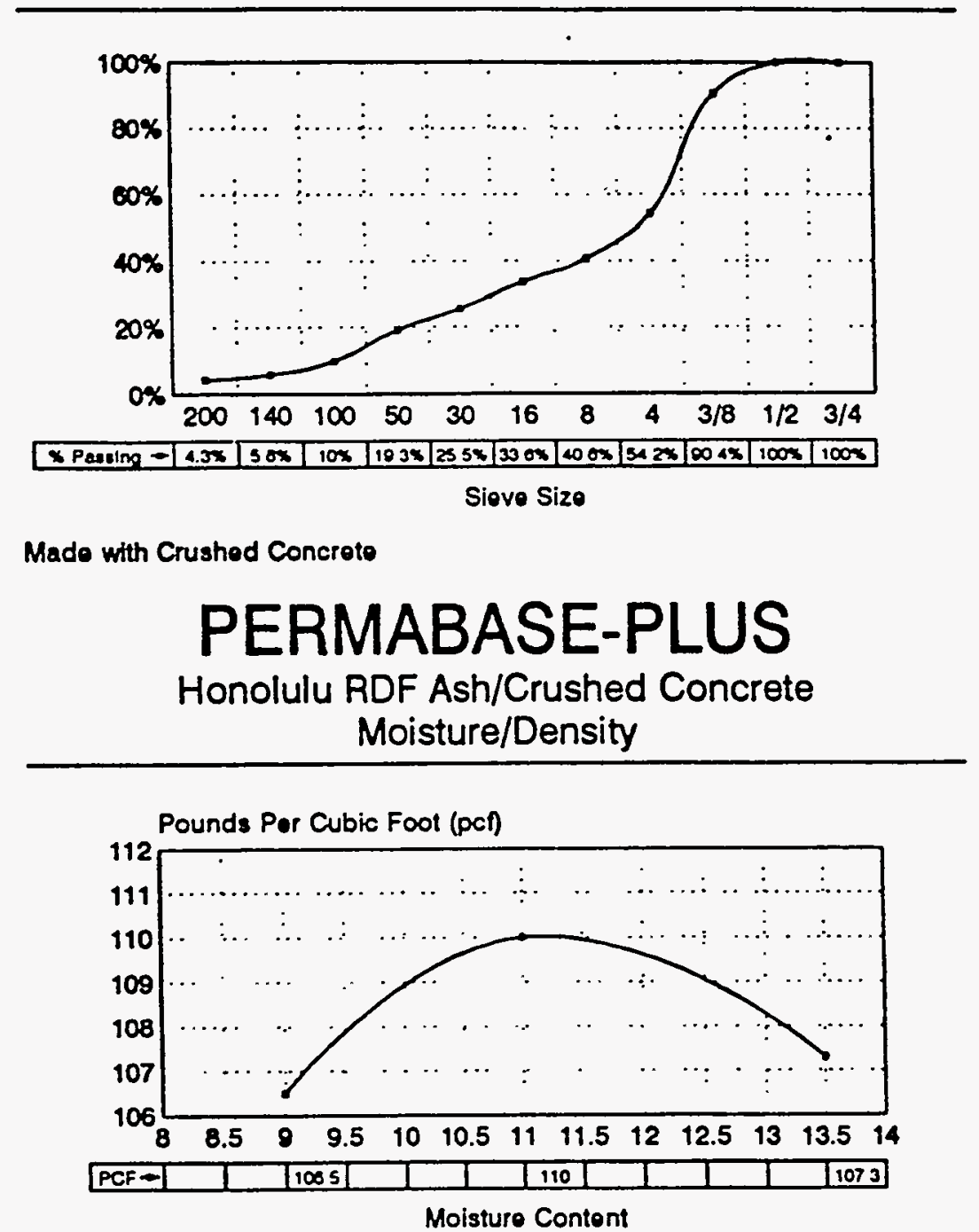

Maximum Density: 110.2 pet

Optimum Moisture: 11.2\%

\section{PERMABASE-PLUS}

Honolulu RDF Ash/Crushed Concrete Compressive Strength

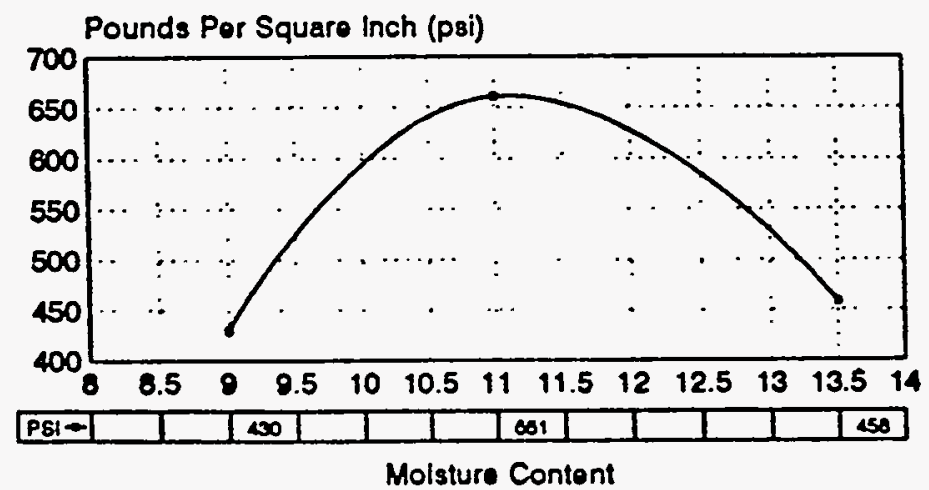

Poak Stross: 661 psl

Peak Moisture: 11.0\% 


\section{PERMABASE-PLUS}

Honolulu RDF Ash/Sand

Sieve Analysis
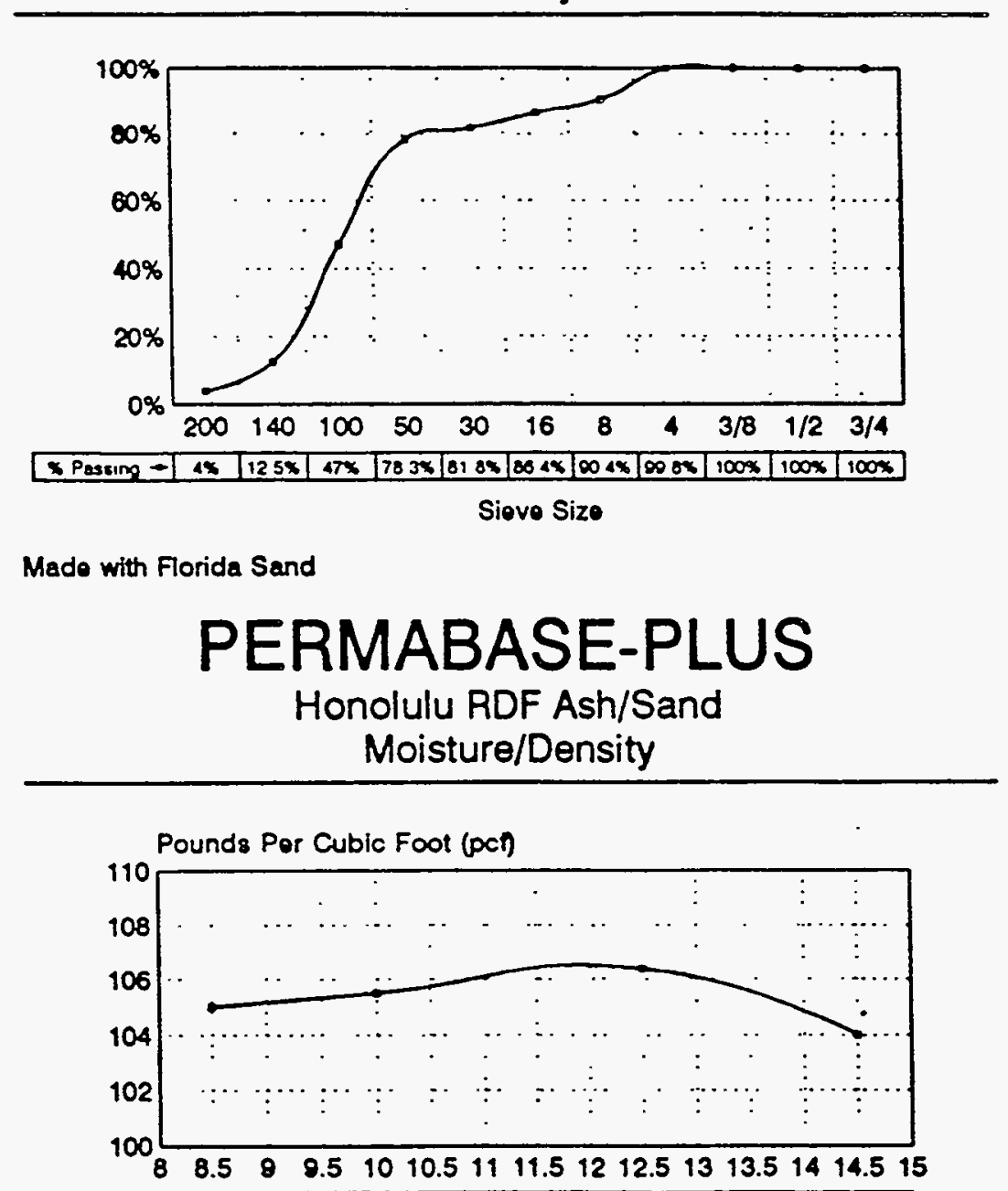

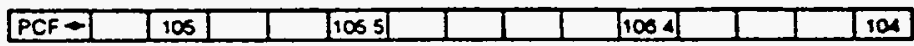

Moisture Content

Maximum Density: 106.5 pof

Optlmum Moisture: 12\%

\section{PERMABASE-PLUS Honolulu RDF Ash/Sand Compressive Strength}

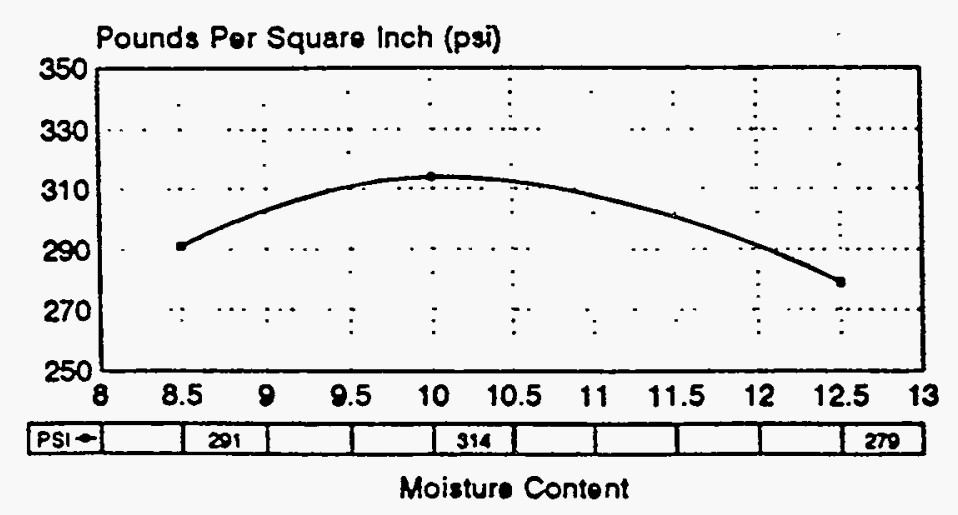


PERMABASE-PLUS

Honolulu Unprocessed Ash

Sieve Analysis

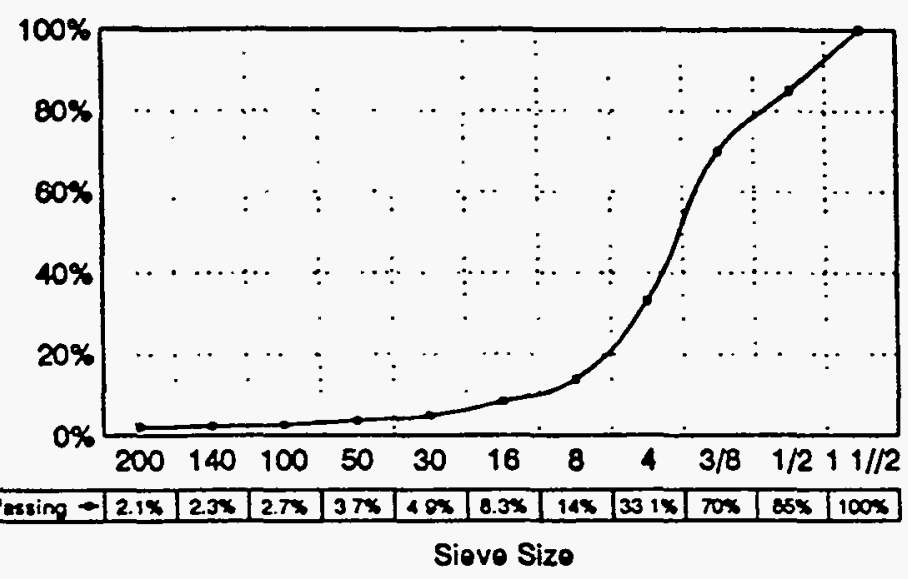

PERMABASE-PLUS

Honolulu Processed Ash

Sieve Analysis

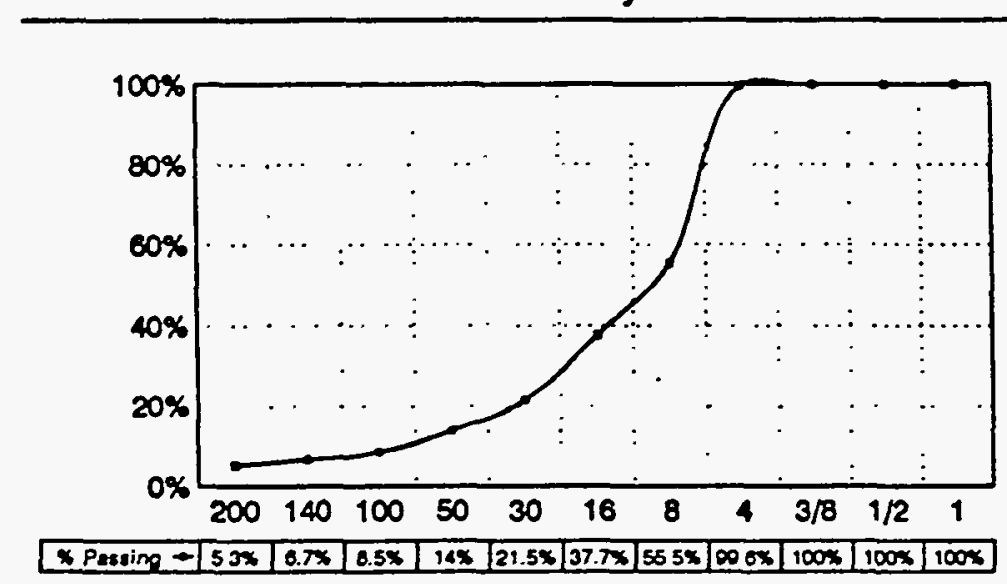

Siove Size 


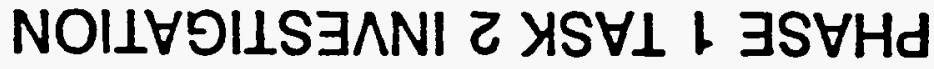

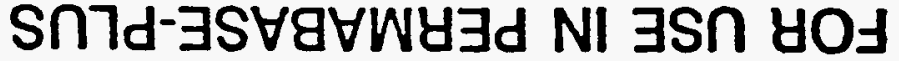 HS $\forall$ NOIISก II $\forall M \forall H ' \cap 7 \cap 7 O N O H$}

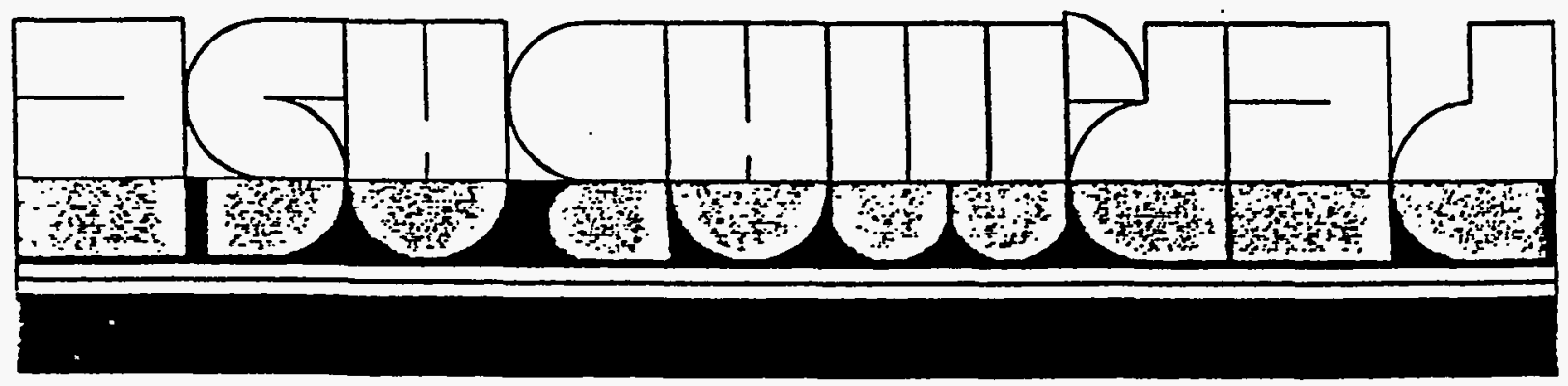

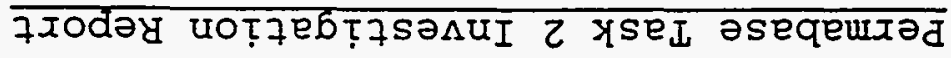

ธg xṬpuədd 


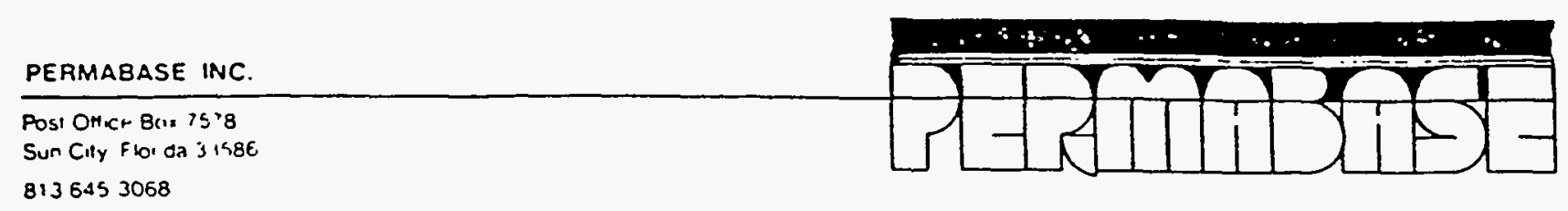

HONOLULU, HAWAII

\author{
MUNICIPAL SOLID WASTE COMBUSTION ASH
}

FOR USE IN PERMABASE-PLUS

PHASE I TASK 2 INVESTIGATION

FEBRUARY, 1994

Permahase, Inc., in cooperation with Ogden Martin Systems Inc., is continuing its participation in the City and County of Honolulu's investigation of Municipal Solid Waste Combustor Ash utilization sponsored by the National Renewable Energy Laboratory (NREL). Several laboratory tests were conducted on H-Power ash and locally available Hawaiian construction materials. The purpose of these tests was to determine the suitability of $\mathrm{H}$-Power ash for use in the patented soil cement material PERMABASE-PLUS while meeting the local Hawaiian Department of Transportation specifications.

On December 28, 1993, the following materials were received by personnel of Permabase, Inc.:

1) One five gallon bucket of H-Power bottom ash

2) One five gallon bucket of $H$-Power combined ash

3) One five gallon bucket of Red Base Rock

4) One five gallon bucket of \#4 Rock

5) One five gallon bucket of \#3 Sand

Items 3, 4 and 5 were provided for the City and County of Honolulu by Grace Pacific Corporation (GP) from their rock quarry and processing facility on Oahu.

PERMABASE-PLUS is a combination of MSW ash, cement, water and a locally produced aggregate. Each of these ingredients can be utilized in varying amounts depending on the quality and availability of the raw materials, and the structural and environmental specifications which apply. The Hawaii Department of Transportation (HDOT), as outlined in the Standard Specifications for Road and Bridge Construction, Section 308 - Portland Cement Treated Base, requires aggregates to be tested by the following procedures: 

Los Angeles Abrasion
Sand Equivalent
Plasticity
Flat or Elungated Pieces
Grading
AASHTO T 96
AASHTO T 176
AASHTO T 90
HW'Y-TC 4
AASHTO T 27

After evaluation of the five materials received and a review of the specifications, Permahase, Inc. decided to utilize GP \#4 Rock and GP \#3 Sand in combination with H-Power combined ash.

The bottom ash was not tested at this time for two reasons: 1) It is the philosophy of Permahase, Inc. to utilize the entire ash stream, reducing the need for separate handling, treatment and disposal; and, 2) the combined ash represents the worst case condition by including the fly ash. As noted below, the fly ash fraction of the ash impacts the structural integrity of a roadway in the Sand Equivalent Test, the Plasticity Test and the Grading Test, while at the same time impacts the environmental tests by introducing a greater potential of leachable inorganic metals. By providing evidence that PERMABASE-PLUS performs satisfactorily with combined ash, it will also provide positive evidence that PERMABASE-PLUS produced with bottom ash only will meet the specifications.

The GP Red Base Rock as received met the grading requirements of the HDOT specification. If more fines were added to this material in the form of ash, it would no longer meet this specification. This would then require a secondary screening operation at the quarry, resulting in a "new" waste product. Therefore, Permabase, Inc. felt it would not be practical to combine ash with Red Rock for these tests.

\section{DISCUSSION OF TESTING PROCEDURES}

The Los Angeles Abrasion procedure tests an aggregate for hardness. Depending upon the grading of the aggregate, certain size fractions (in this case the plus $1 / 2$ inch) of the aggregate are placed in a tumbler with steel balls and rotated 500 times. The size fraction of the aggregate is then removed and screened to determine the amount of material which was crushed by the steel balls. Due to the HDOT grading specifications and the necessity to crush the ash to a minus $3 / 8$ inch size before use in the PERMABASE-PLUS aggregate, the ash itself does not impact the results of the Los Angeles Abrasion. However, it is necessary to select an aggregate when designing the PERMABASE-PLUS aggtegate which will meet the requirements of this rigorous test.

The Sand Equivalent procedure tests an aggregate for suspended fines. The procedure defines these suspended fines as clay and dust and is conducted on the minus $\# 4$ sieve $(4.75 \mathrm{~mm})$ fraction of the aggregate. The sand equivalent number is determined through a precise procedure as a ratio between the particles which settle within a solution and those that do not. Since all the ash is crushed to a minus $3 / 8$ inch sieve in the PERMABASE-PLUS procedure, the ash will directly impact these test results. Although there are no clays in the ash, the dust particles - typically the minus \#200 (75 microns) sieve material - will remain in suspension. 
The Plasticity procedure tests an aggregate for its ahility to retain water. The procedure is conducted on the minus $\# 40$ (425 micron) sieve fraction of the aggregate. The ash will make up a large portion of this fraction, and will impact the test results.

The Flat or Elongated Pieces procedure tests for detrimental shapes in aggregates. This procedure is conducted on the plus $3 / 8$ inch fraction of the aggregate. Again because the ash is crushed to a minus $3 / 8$ inch before use in PERMABASE-PLUS, the ash will not impact these test results.

The Grading Procedure tests an aggregate for its grain size distribution. By controlling size distribution, this specification impacts all the above tests and in turn controls the quality of the aggregate. The HDOT " $3 / 4$ Inch Maximum" aggregate requires that at least $45 \%$ of the aggregate is larger than a $\# 4(4.75 \mathrm{~mm})$ sieve. This in turn controls the Los Angeles Abrasion grading selection and the Flat and Elongated Pieces Procedure. This same specification also limits the minus $\$ 200$ (75 micron) sieve portion to a maximum of $9 \%$. This in turn impacts the Sand Equivalent and Plasticity test results.

\section{ADDITIONAL AGGREGATES}

Two PERMABASE-PLUS aggregates were also prepared utilizing H-Power combined ash and a Florida crushed concrete (CC). This combination offers an opportunity for the City and County of Honolulu to not only recycle combustor ash, but also a demolition debris currently being landfilled. Although not commercially available at this time, Permabase, Inc. felt that this option may at some time become available in Honolulu.

\section{AGGREGATE DESIGN PROPORTIONS}

Many combinations of aggregate and ash can be created in differing proportions to create PERMABASE-PLUS aggregate. Due to sample size and time limitations, Permabase, Inc. chose to prepare four aggregate combinations. Permabase, Inc. selected a minimum content of $25 \%$ ash as a practical level of recycling necessary for commercial viability, and a maximum of $50 \%$ ash due to HDOT specification limits. If commercial application is warranted, the "fine tuning" of practice and experience will determine the optimum aggregate combination. The four aggregates were prepared for testing in the following combinations:

Aggregate \#1: $\quad 50 \%$ GP \#4 Rock

$25 \%$ GP \#3 Sand

25\% H-Power Combined Ash

Aggregate $\quad 52: \quad 50 \%$ GP \#4 Rock

$50 \%$ H-Power Combined Ash 
Aggregate \#3: $\quad$ 50\% Coarse Graded Crushed Concrete 25\% Fine Graded Fine Graded Concrete 25\% H-Power Combined Ash

Aggregate \#4: $\quad 50 \%$ Coarse Graded Crushed Concrete 50\% H-Power Combined Ash

NOTE: Coarse graded aggregates are larger than the \#4 $(4.75 \mathrm{~mm})$ sieve.

Results of HDOT testing procedures appear on the enclosed Lahoratory Test Results matrix.

\section{CONCLUSION}

Processed H-Power ash can be successfully combined with locally available natural aggregates or recycled materials to meet all specifications as detailed in Section 308 - Portland Cement Treated Base, of the Hawaii Department of Transportation Standard Specifications for Road and Bridge Construction.

\section{RECOMMENDATION}

Phase II of the NREL sponsored test protocol will include a large scale field study at the current County landfill. For this demonstration, Permabase, Inc. recommends the PERMABASE-PLUS Aggregate combination \#1 - 50\% GP \#4 Rock, 25\% GP \#3 Sand and 25\% H-Power Combined Ash. These materials are readily available and will ensure a structurally sound, easily workable aggregate mix for the demonstration. The 50\% ash combination is not recommended at this time due to sensitivity of moisture content near optimum moisture. In the laboratory, the mixture with $50 \%$ ash tends to become too fluid, too quickly once optimum moisture is attained. This characteristic may be controlled by the use of more absorbent aggregates or possibly by removing part of the fly ash, but for the purposes of Phase II, should not be used. 


\section{rEKIVIABASE-rLUS \\ Honolulu MSW Ash/Various Aggregates Laboratory Test Results}

\begin{tabular}{|c|c|c|c|c|c|}
\hline Test Method & Specification & Aggregate 1 & Aggregate 2 & Aggregate 3 & Aggregate 4 \\
\hline L.A. Abrasion & $50 \% \operatorname{Max}$ & $9.7 \%$ & $9.7 \%$ & $48.3 \%$ & $48.3 \%$ \\
\hline \multicolumn{6}{|l|}{ AASHTO T 96} \\
\hline Sand Equivalent & $35 \%$ Min & $68.3 \%$ & $71.9 \%$ & $70 \%$ & $71.9 \%$ \\
\hline \multicolumn{6}{|l|}{ AASHTO T 176} \\
\hline Plasticity Index & $6 \operatorname{Max}$ & Non Plastic & Non Plastic & Non Plastic & Non Plastic \\
\hline \multicolumn{6}{|l|}{ AASHTO T 90} \\
\hline Flat or Elongated & $25 \% \operatorname{Max}$ & $3.1 \%$ & $3.1 \%$ & $5.3 \%$ & $5.3 \%$ \\
\hline \multicolumn{6}{|l|}{ Pieces } \\
\hline \multicolumn{6}{|l|}{ HWY-TC4 } \\
\hline \multicolumn{6}{|l|}{ Grading } \\
\hline AASHTO T 27 & Passing & & & & \\
\hline $1 "$ Sieve & $100 \%$ & $100 \%$ & $100 \%$ & $100 \%$ & $100 \%$ \\
\hline 3/4" Sieve & $90-100 \%$ & $92 \%$ & $91 \%$ & $100 \%$ & $100 \%$ \\
\hline \#4 Sieve & $35-55 \%$ & $49 \%$ & $51 \%$ & $50 \%$ & $50 \%$ \\
\hline \#200 Sieve & $3-9 \%$ & $4.8 \%$ Table 1 & $4.7 \%$ & $4.1 \%$ & $3.9 \%$ \\
\hline
\end{tabular}



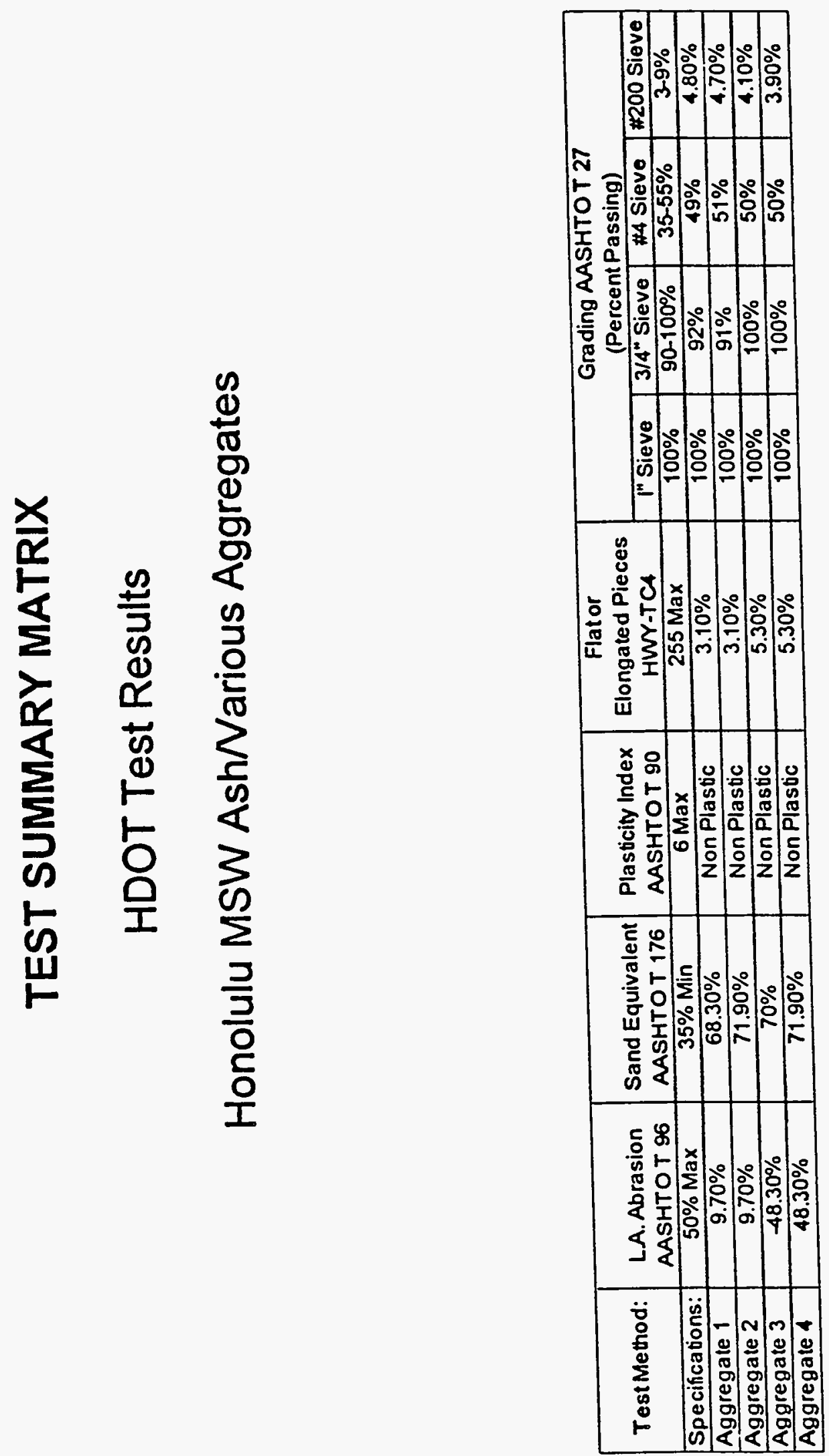

실 


\title{
THE ENVIRONMENTAL CHARACTERIZATION OF RDF COMBUSTION TECHNOLOGY
}

\author{
Mid-Connecticut Facility \\ Hartford, Connecticut
}

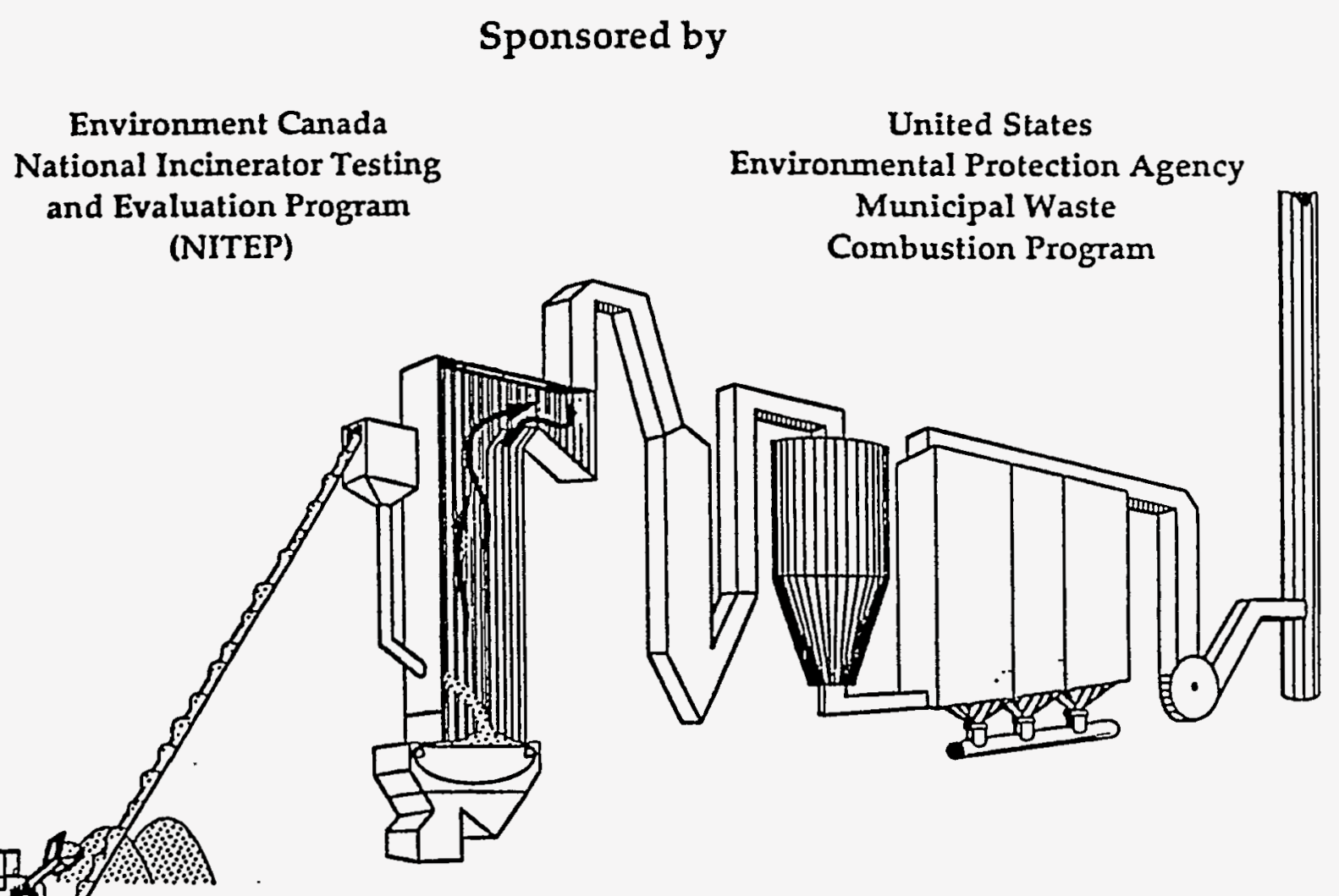

Volume II

Test Program and Results

April 1991 


\title{
THE ENVIRONMENTAL CHARACTERIZATION \\ OF \\ RDF COMBUSTION TECHNOLOGY
}

Mid-Connecticut Facility

Hartord, Connecticut

VOLUME II

TEST PROGRAM AND RESULTS

\author{
Compiled by
}

Alliance Technologies Corporation

Lowell, Massachusetts

for

Environment Canada

National Incinerator Testing

And Evaluation Program

(NITEP)

\author{
United States \\ Environmental Protection Agency \\ Municipal Waste \\ Combustion Program
}

This report has been reviewed by Environment Canada and the U.S. EPA. The contents do not necessarily reflect the views and policies of these agencies. Mention of trade names or commercial products does not constitute endorsement for use.

Any comments concerning its content should be directed to:

NITEP

Industrial Programs Branch

Urban Activities Division

Conservation and Protection

Environment Canada

Ottawa, Ontario

$\mathrm{K} 1 \mathrm{~A} \mathrm{OH} 3$
Air and Energy Engineering Research

Laboratory

U.S. Environmental Protection Agency

Research Triangle Park, North Carolina 27711

April 1991 
Table 7-14. Dry Bottom Ash or Data for Hetals

\begin{tabular}{|c|c|c|c|c|c|c|c|c|c|c|c|}
\hline \multirow{4}{*}{ AKALYTE } & \multirow{4}{*}{$\begin{array}{l}\text { PT-06 } \\
(\mathrm{mg} / \mathrm{kg})\end{array}$} & \multirow{4}{*}{$\begin{array}{l}\text { DUPL } \\
(\mathrm{mg} / \mathrm{kg})\end{array}$} & \multirow{4}{*}{$\begin{array}{l}\text { RPD } \\
(x)\end{array}$} & \multirow{4}{*}{$\begin{array}{c}\text { PT-11 } \\
(\mathrm{mg} / \mathrm{kg})\end{array}$} & \multirow{4}{*}{$\begin{array}{l}\text { DUPL } \\
\text { (mg/kg) }\end{array}$} & \multirow{4}{*}{$\begin{array}{ll}\text { RPD } & 1 \\
\text { (x) } & \text { I }\end{array}$} & \multirow{4}{*}{$\begin{array}{c}\text { PT-06 } \\
\text { (mg/kg) }\end{array}$} & \multirow{4}{*}{$\begin{array}{l}\text { Conc. } \\
\text { Spiked } \\
(\mathrm{mg} / \mathrm{kg})\end{array}$} & \multirow{4}{*}{$\begin{array}{l}\text { PT-06/ } \\
\text { Spike } \\
\text { Ratio }\end{array}$} & \multirow{4}{*}{\multicolumn{2}{|c|}{$\begin{array}{l}\text { Spiked } \\
\text { Sample Recovery } \\
\text { (mg/kg) }\end{array}$}} \\
\hline & & & & & & & & & & & \\
\hline & & & & & & & & & & & \\
\hline & & & & & & & & & & & \\
\hline CNDIUA & 4.6 & 5.8 & $23 x 1$ & 6.8 & 6.9 & $|x|$ & 4.6 & 2500 & 0 & 2300 & $92 \times 1$ \\
\hline DERYLLILA & - & $<1$ & 1 & $\bullet$ & $<1$ & i & $\bullet$ & 2500 & & 2400 & $96 \% 1$ \\
\hline CALCIUA & 75643 & 89100 & $16 \%$ & 80687 & 96700 & $18 x \mid$ & 75643 & 2500 & 30 & 92000 & $C T * 1$ \\
\hline VAHADIUK & 105 & 120 & $93 \times 1$ & 68 & 75.2 & $90 x 1$ & 105 & 2500 & 0 & 2590 & $9 \% 1$ \\
\hline ALCHINUM & 58396 & 66100 & $12 \% 1$ & 47439 & 56800 & $18 x \mid$ & 58396 & 2500 & 23 & 69000 & $C T=1$ \\
\hline MAGKESIUH & 7704 & 9200 & $18 \% 1$ & 7785 & 10600 & $39 x \mid$ & 7704 & 2500 & 3 & 11700 & $160 \% 1$ \\
\hline MARIUH & 403 & 470 & $15 \times 1$ & 187 & 240 & $25 \times 1$ & 403 & 2500 & 0 & 3050 & $106 \% 1$ \\
\hline zInC & 1261 & 1450 & $14 x \mid$ & 1370 & 1830 & 2971 & 1261 & 2500 & 1 & 3860 & $104 \times 1$ \\
\hline МИGAKESE & 499 & 550 & $10 \% \mid$ & 511 & 690 & $30 \% 1$ & 499 & 2500 & 0 & 2850 & $94 \times 1$ \\
\hline COBALT & 22 & 27.4 & $22 \% 1$ & 24 & 45.7 & 6271 & 22 & 2500 & 0 & 2300 & $99 \times 1$ \\
\hline COPPER & 1121 & 1300 & $15 \times 1$ & 4882 & 6000 & $21 x \mid$ & 1121 & 2500 & 0 & 3600 & $99 \% 1$ \\
\hline IRON & 14796 & 16400 & $10 \times 1$ & 25301 & 32600 & $25 \times 1$ & 14796 & 2500 & 6 & 19100 & $972 x \mid$ \\
\hline LEND & 1016 & 1170 & $14 \times 1$ & 2254 & 3000 & $28 \times 1$ & 1016 & 2500 & 0 & 3500 & $99 \times 1$ \\
\hline CHROMILAY & 158 & 160 & $|\mathbf{x}|$ & 170 & 270 & $45 \times 1$ & 158 & 2500 & 0 & 2400 & $90 \times 1$ \\
\hline MICKEL & 96 & 100 & $4 x 1$ & 243 & 370 & $6|x|$ & 96 & 2500 & 0 & 2900 & $112 \times 1$ \\
\hline SILVER & - & $<1$ & 1 & $\bullet$ & $<1$ & 1 & - & 2500 & & 2550 & $102 \%$ \\
\hline PHOSPKORUS & 78 & 68.7 & $-13 \% \mid$ & 389 & 540 & $33 \times 1$ & 78 & 2500 & 0 & 2800 & $108 x$ \\
\hline soolun & 48765 & 66000 & $30 \% 1$ & 54251 & 63000 & $15 \times 1$ & 48765 & 2500 & 20 & 68000 & $c T=1$ \\
\hline EISNETH : & 385 & 560 & $37 x 1$ & 787 & 1200 & $42 \times 1$ & 385 & 2500 & 0 & 3060 & $107 x 1$ \\
\hline IHOIUH : & - & $<q$ & 1 & - & $<1$ & 1 & - & 2500 & & 2500 & $100 \%$ \\
\hline MOLYBDEKUM & 15 & 17.9 & $18 \%$ & 12 & 14.9 & 2221 & 15 & 2500 & 0 & 2780 & $191 \% 1$ \\
\hline TIH & 394 & 400 & $2 \times 1$ & 235 & 310 & $28 \% 1$ & 394 & 2500 & 0 & 2910 & $109 \% 1$ \\
\hline SILICOH & $437 \pi s$ & 51500 & $16 \% 1$ & 48817 & 63000 & $25 \times 1$ & 43775 & 2500 & 18 & 52800 & $C T=1$ \\
\hline TITAKIUM & 7967 & 9300 & $15 \times 1$ & 5271 & 6000 & $13 x 1$ & 7967 & 2500 & 3 & 11800 & $\mid 53 \% 1$ \\
\hline ARSEHIC & 7.8 & 8.6 & $10 \% 1$ & 6.5 & 8.25 & $24 x 1$ & 7.8 & 25 & 0 & 34.1 & $105 \% 1$ \\
\hline SELEKIUM & - & $<2.5$ & & 0.8 & 0.9 & $|27|$ & - & 25 & & 25.9 & $106 x 1$ \\
\hline АКTIКОНY & - & $<1.25$ & 1 & 2.2 & 2.6 & $177 x$ & - & 25 & & 29.7 & $119 \% 1$ \\
\hline TELLURIUM & - & $<2.5$ & 1 & 3.5 & 3.89 & $\mid 172$ & - & 25 & & 31.3 & $125 \%$ \\
\hline MERQURY & 0.322 & & & - & & & 0.322 & & & & \\
\hline
\end{tabular}

-CT = Camot test for pereent recovery on spiked samples when

original sample concentration is more than 10 times spike concentration. 
Table 7-16. Fobric Filter Ash Sample OC Data for Hetals (continued)

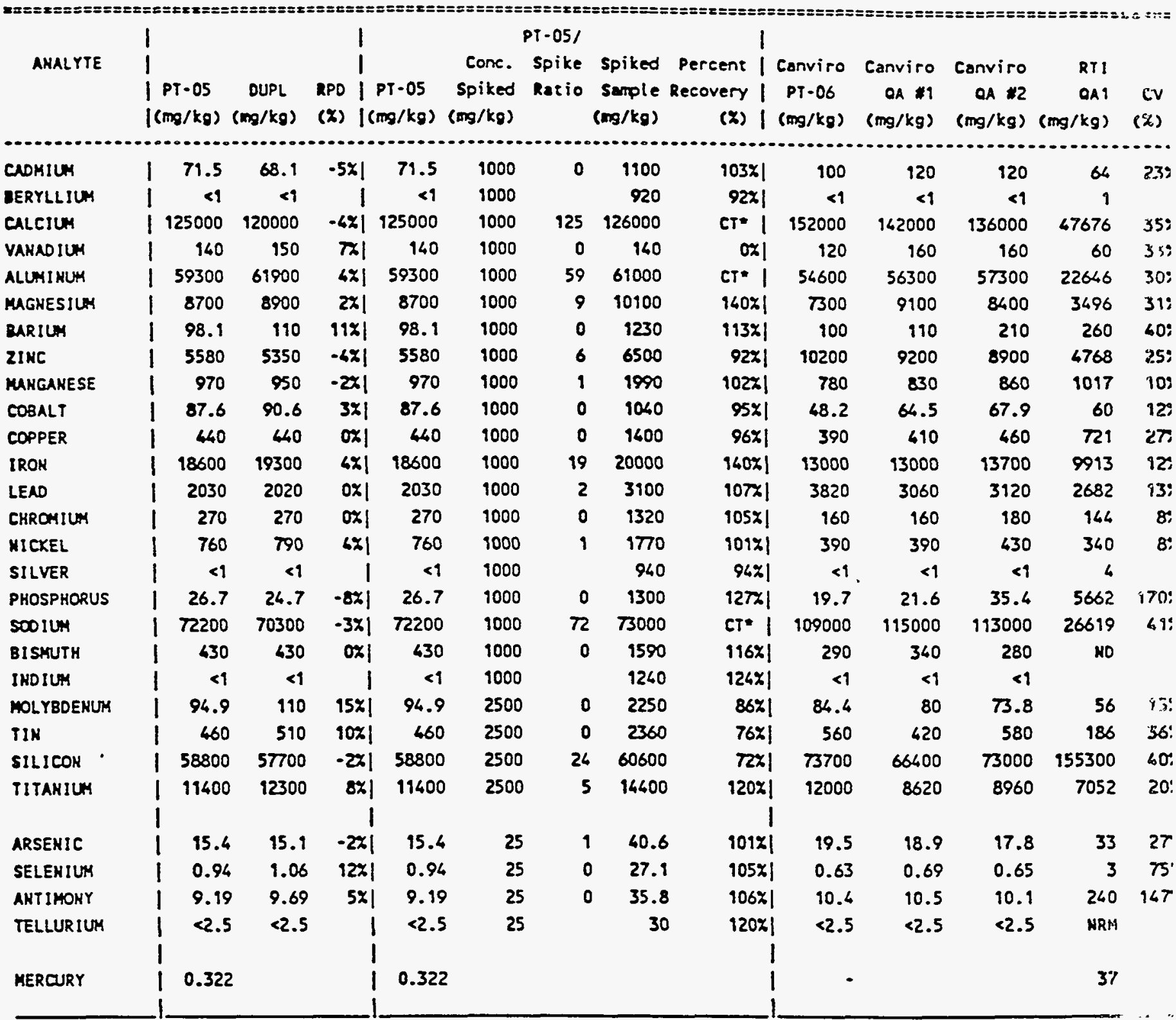

- CT = Camnot test for percent recovery on spiked samples when original sample concentration is more than 10 times spike concentration. 
TABLE 9-5. PERFORMANCE TEST DATA SUMARRY: ASH ORGAMICMETALS

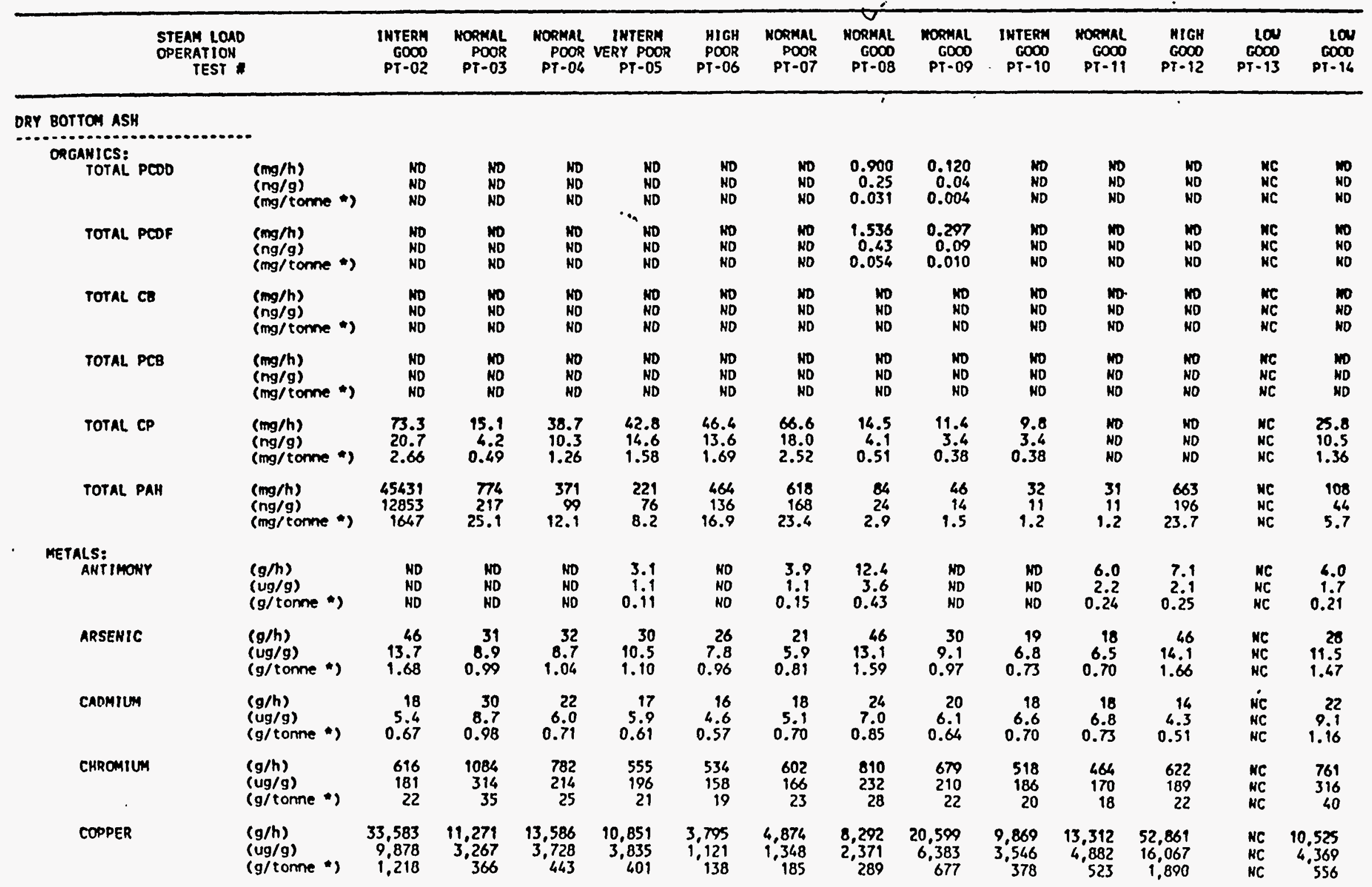


TABLE 9-5. PERFormance tEST DATA sUMAARY: ASH ORGANIC/METALS (Continued)

\begin{tabular}{|c|c|c|c|c|c|c|c|c|c|c|c|c|c|c|}
\hline \multicolumn{2}{|c|}{$\begin{array}{c}\text { STEAM LOAD } \\
\text { OPERATION } \\
\text { TEST }\end{array}$} & \multirow{2}{*}{$\begin{array}{r}\begin{array}{r}\text { INTERM } \\
\text { GO00 } \\
\text { PY }-02\end{array} \\
3.218 \\
947 \\
117\end{array}$} & \multirow{2}{*}{$\begin{array}{r}\begin{array}{r}\text { NORMAL } \\
\text { POOR } \\
\text { PT }-03\end{array} \\
\begin{array}{r}5,500 \\
1,594 \\
179\end{array}\end{array}$} & \multirow{2}{*}{$\begin{array}{r}\begin{array}{r}\text { NORMAL } \\
\text { POOR } \\
\text { PT }-04\end{array} \\
\begin{array}{r}5,050 \\
1,386 \\
165\end{array}\end{array}$} & \multirow{2}{*}{$\begin{array}{c}\begin{array}{c}\text { INTERM } \\
\text { VERY POOR } \\
\text { PT }-05\end{array} \\
\begin{array}{c}5,414 \\
1.913 \\
200\end{array}\end{array}$} & \multirow{2}{*}{$\begin{array}{r}\text { HIGH } \\
\text { POOR } \\
\text { PT-06 }\end{array}$} & \multirow{2}{*}{$\begin{array}{r}\begin{array}{r}\text { MORMAL } \\
\text { POOR } \\
\text { PT-07 }\end{array} \\
\begin{array}{r}6,590 \\
1.823 \\
249\end{array}\end{array}$} & \multirow{2}{*}{$\begin{array}{r}\begin{array}{r}\text { NORMAL } \\
\text { GO00 } \\
\text { PT-08 }\end{array} \\
\begin{array}{r}13,099 \\
3,746 \\
457\end{array}\end{array}$} & \multirow{2}{*}{$\begin{array}{r}\begin{array}{r}\text { NORMAL } \\
\text { GO00 } \\
\text { PT-09 }\end{array} \\
\begin{array}{r}3,864 \\
1,197 \\
127\end{array}\end{array}$} & \multirow{2}{*}{$\begin{array}{r}\begin{array}{r}\text { INTERM } \\
\text { GOO0 } \\
\text { PT- } 10\end{array} \\
\begin{array}{r}4.303 \\
1.546 \\
165\end{array}\end{array}$} & \multirow{2}{*}{$\begin{array}{r}\begin{array}{r}\text { YORMAL } \\
\text { GOOO } \\
\text { PT }-11\end{array} \\
\begin{array}{r}6,147 \\
2,254 \\
242\end{array}\end{array}$} & \multirow{2}{*}{ 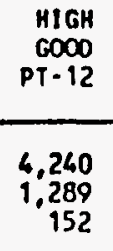 } & \multirow{2}{*}{$\begin{array}{r}\begin{array}{r}\text { LON } \\
\text { COOD } \\
\text { PT- } 13\end{array} \\
\begin{array}{r}\text { NC } \\
\text { NC } \\
\text { NC }\end{array}\end{array}$} & \multirow{2}{*}{$\begin{array}{r}\text { LOW } \\
\text { GO00 } \\
\text { PT-14 } \\
\begin{array}{r}8.675 \\
3.601 \\
458\end{array}\end{array}$} \\
\hline LEAD & $\begin{array}{l}(g / h) \\
\text { (ug/g) } \\
\text { (g/tonne *) }\end{array}$ & & & & & & & & & & & & & \\
\hline MERCURY & $\begin{array}{l}(g / h) \\
\text { (ug/g) } \\
\text { (g/tonne }\end{array}$ & $\begin{array}{l}\text { NO } \\
\text { NO } \\
\text { ND }\end{array}$ & $\begin{array}{l}\text { NO } \\
\text { NO } \\
\text { NO }\end{array}$ & $\begin{array}{l}0.130 \\
0.035 \\
0.004\end{array}$ & $\begin{array}{l}\text { ND } \\
\text { ND } \\
\text { ND }\end{array}$ & $\begin{array}{l}1.090 \\
0.322 \\
0.040\end{array}$ & $\begin{array}{l}0.980 \\
0.272 \\
0.037\end{array}$ & $\begin{array}{l}\text { ND } \\
\text { ND } \\
\text { ND }\end{array}$ & $\begin{array}{l}\text { HO } \\
\text { HD } \\
\text { HO }\end{array}$ & $\begin{array}{l}0.230 \\
0.081 \\
0.009\end{array}$ & $\begin{array}{l}\text { ND } \\
\text { ND } \\
\text { ND }\end{array}$ & $\begin{array}{l}0.085 \\
0.026 \\
0.003\end{array}$ & $\begin{array}{l}\text { NC } \\
\text { NC } \\
\text { NC }\end{array}$ & $\begin{array}{l}\text { NO } \\
\text { NO } \\
\text { NO }\end{array}$ \\
\hline NICKEL & $\begin{array}{l}(g / h) \\
(u g / g) \\
(g / \text { tonne *) }\end{array}$ & $\begin{array}{r}1.931 \\
568 \\
70\end{array}$ & $\begin{array}{r}867 \\
251 \\
28\end{array}$ & $\begin{array}{r}631 \\
173 \\
21\end{array}$ & $\begin{array}{r}833 \\
294 \\
31\end{array}$ & $\begin{array}{r}326 \\
96 \\
12\end{array}$ & $\begin{array}{r}331 \\
92 \\
13\end{array}$ & $\begin{array}{r}594 \\
170 \\
21\end{array}$ & $\begin{array}{r}705 \\
218 \\
23\end{array}$ & $\begin{array}{r}293 \\
105 \\
11\end{array}$ & $\begin{array}{r}663 \\
243 \\
26\end{array}$ & $\begin{array}{r}565 \\
172 \\
20\end{array}$ & $\begin{array}{l}\text { NC } \\
\text { NC } \\
\text { NC }\end{array}$ & $\begin{array}{r}802 \\
333 \\
42\end{array}$ \\
\hline ZINC & $\begin{array}{l}(g / h) \\
(u g / g) \\
\text { (g/torne *) }\end{array}$ & $\begin{array}{l}4,813 \\
1,416 \\
175\end{array}$ & $\begin{array}{r}3,414 \\
990 \\
111\end{array}$ & $\begin{array}{r}4,539 \\
1,245 \\
148\end{array}$ & $\begin{array}{r}3,262 \\
1,153 \\
121\end{array}$ & $\begin{array}{r}4,269 \\
1,261 \\
155\end{array}$ & $\begin{array}{r}4,935 \\
1,365 \\
187\end{array}$ & $\begin{array}{r}5,942 \\
1,699 \\
207\end{array}$ & $\begin{array}{l}3,603 \\
1,116 \\
118\end{array}$ & $\begin{array}{l}5,092 \\
1,830 \\
195\end{array}$ & $\begin{array}{r}3,737 \\
1,370 \\
147\end{array}$ & $\begin{array}{r}3,618 \\
1,100 \\
129\end{array}$ & $\begin{array}{l}\text { NC } \\
\text { RC } \\
\text { NC }\end{array}$ & $\begin{array}{r}6.523 \\
1.877 \\
239\end{array}$ \\
\hline \multicolumn{15}{|c|}{ GRATE SIFTINGS ASH } \\
\hline ANTIMONY & $\begin{array}{l}(g / h) \\
\text { (ug/g) } \\
\text { (g/torne *) }\end{array}$ & $\begin{array}{r}5.75 \\
41 \\
0.209\end{array}$ & $\begin{array}{r}3.81 \\
30 \\
0.124\end{array}$ & $\begin{array}{r}5.60 \\
53 \\
0.183\end{array}$ & $\begin{array}{r}2.62 \\
25 \\
0.097\end{array}$ & $\begin{array}{r}3.13 \\
44 \\
0.114\end{array}$ & $\begin{array}{r}5.91 \\
51 \\
0.224\end{array}$ & $\begin{array}{r}0.61 \\
6.1 \\
0.021\end{array}$ & $\begin{array}{r}6.96 \\
59 \\
0.229\end{array}$ & $\begin{array}{r}1.03 \\
11 \\
0.039\end{array}$ & $\begin{array}{r}4.96 \\
45 \\
0.195\end{array}$ & $\begin{array}{r}2.47 \\
23 \\
0.088\end{array}$ & $\begin{array}{l}\text { NC } \\
\text { NC } \\
\text { NC }\end{array}$ & $\begin{array}{r}1.87 \\
21 \\
0.098\end{array}$ \\
\hline ARSENIC & $\begin{array}{l}(g / h) \\
\text { (ug/g) } \\
\text { (g/tonne *) }\end{array}$ & $\begin{array}{r}1.84 \\
13.2 \\
0.067\end{array}$ & $\begin{array}{r}1.00 \\
8.0 \\
0.033\end{array}$ & $\begin{array}{r}0.83 \\
7.9 \\
0.027\end{array}$ & $\begin{array}{r}0.84 \\
8.1 \\
0.031\end{array}$ & $\begin{array}{r}0.67 \\
9.4 \\
0.024\end{array}$ & $\begin{array}{r}1.19 \\
10.3 \\
0.045\end{array}$ & $\begin{array}{r}0.97 \\
9.7 \\
0.034\end{array}$ & $\begin{array}{r}1.58 \\
13.4 \\
0.052\end{array}$ & $\begin{array}{r}0.70 \\
7.7 \\
0.027\end{array}$ & $\begin{array}{r}1.06 \\
9.7 \\
0.042\end{array}$ & $\begin{array}{r}1.39 \\
13.1 \\
0.050\end{array}$ & $\begin{array}{l}\text { NC } \\
\text { NC } \\
\text { NC }\end{array}$ & $\begin{array}{r}0.86 \\
9.7 \\
0.046\end{array}$ \\
\hline CADMIUM & $\begin{array}{l}(g / h) \\
(u g / g) \\
(g / \text { tonne *) }\end{array}$ & $\begin{array}{r}0.88 \\
6.3 \\
0.032\end{array}$ & $\begin{array}{r}1.02 \\
8.1 \\
0.033\end{array}$ & $\begin{array}{r}1.30 \\
12.4 \\
0.042\end{array}$ & $\begin{array}{r}1.16 \\
11.3 \\
0.043\end{array}$ & $\begin{array}{r}0.82 \\
11.5 \\
0.030\end{array}$ & $\begin{array}{r}1.32 \\
11.4 \\
0.050\end{array}$ & $\begin{array}{r}1.29 \\
12.9 \\
0.045\end{array}$ & $\begin{array}{r}0.63 \\
5.3 \\
0.021\end{array}$ & $\begin{array}{r}1.02 \\
19.2 \\
0.039\end{array}$ & $\begin{array}{r}1.33 \\
12.1 \\
0.052\end{array}$ & $\begin{array}{r}1.38 \\
13.0 \\
0.049\end{array}$ & $\begin{array}{l}\text { NC } \\
\text { NC } \\
\text { NC }\end{array}$ & $\begin{array}{r}0.78 \\
8.8 \\
0.049\end{array}$ \\
\hline CHROMIUN & $\begin{array}{l}(g / h) \\
\text { (ug/g) } \\
\text { (g/tonne *) }\end{array}$ & $\begin{array}{r}60 \\
430 \\
2.18\end{array}$ & $\begin{array}{r}29 \\
233 \\
0.94\end{array}$ & $\begin{array}{r}48 \\
461 \\
1.58\end{array}$ & $\begin{array}{r}47 \\
454 \\
1.73\end{array}$ & $\begin{array}{r}20 \\
284 \\
0.73\end{array}$ & $\begin{array}{r}37 \\
319 \\
1.40\end{array}$ & $\begin{array}{r}21 \\
208 \\
0.73\end{array}$ & $\begin{array}{r}39 \\
328 \\
1.27\end{array}$ & $\begin{array}{r}35 \\
388 \\
1.36\end{array}$ & $\begin{array}{r}34 \\
311 \\
1.34\end{array}$ & $\begin{array}{r}20 \\
192 \\
0.73\end{array}$ & $\begin{array}{l}\text { NC } \\
\text { HC } \\
\text { NC }\end{array}$ & $\begin{array}{r}26 \\
297 \\
1.39\end{array}$ \\
\hline COPPER & $\begin{array}{l}(g / h) \\
\text { (ug/g) } \\
\text { (g/tonne*) }\end{array}$ & $\begin{array}{r}307 \\
2,196 \\
11.1\end{array}$ & $\begin{array}{r}93 \\
745 \\
3.0\end{array}$ & $\begin{array}{r}218 \\
2,079 \\
7.1\end{array}$ & $\begin{array}{r}98 \\
956 \\
3.6\end{array}$ & $\begin{array}{r}819 \\
11,534 \\
29.8\end{array}$ & $\begin{array}{r}208 \\
1.797 \\
7.9\end{array}$ & $\begin{array}{r}156 \\
1.563 \\
5.5\end{array}$ & $\begin{array}{r}192 \\
1.630 \\
6.3\end{array}$ & $\begin{array}{r}1,505 \\
16,533 \\
57.7\end{array}$ & $\begin{array}{r}420 \\
3,819 \\
16.5\end{array}$ & $\begin{array}{r}171 \\
1.616 \\
6.1\end{array}$ & $\begin{array}{l}\text { HC } \\
\text { HC } \\
\text { HC }\end{array}$ & $\begin{array}{r}352 \\
3,958 \\
98.6\end{array}$ \\
\hline LEAD & $\begin{array}{l}(g / h) \\
\text { (ug/g) } \\
\text { (g/tonne*) }\end{array}$ & $\begin{array}{r}2,869 \\
20,494 \\
104.0\end{array}$ & $\begin{array}{r}268 \\
2.142 \\
8.7\end{array}$ & $\begin{array}{r}522 \\
4,971 \\
17.0\end{array}$ & $\begin{array}{r}400 \\
3,881 \\
14.8\end{array}$ & $\begin{array}{r}1,195 \\
16,829 \\
43.4\end{array}$ & $\begin{array}{r}1,859 \\
16,022 \\
70.4\end{array}$ & $\begin{array}{r}443 \\
4.429 \\
15.5\end{array}$ & $\begin{array}{r}2,084 \\
17,665 \\
68.5\end{array}$ & $\begin{array}{r}482 \\
5,294 \\
18.5\end{array}$ & $\begin{array}{r}782 \\
7,105 \\
30.7\end{array}$ & $\begin{array}{r}907 \\
8,558 \\
32.4\end{array}$ & $\begin{array}{l}\text { KC } \\
\text { KC } \\
\text { NC }\end{array}$ & $\begin{array}{r}760 \\
8,545 \\
40.2\end{array}$ \\
\hline MERCURY & $\begin{array}{l}(g / h) \\
(u g / g) \\
(g / \text { tonne })\end{array}$ & $\begin{array}{l}0.127 \\
0.910 \\
0.005\end{array}$ & $\begin{array}{l}0.293 \\
2.350 \\
0.010\end{array}$ & $\begin{array}{l}0.201 \\
1.920 \\
0.007\end{array}$ & $\begin{array}{l}0.208 \\
2.020 \\
0.008\end{array}$ & $\begin{array}{l}0.072 \\
1.020 \\
0.003\end{array}$ & $\begin{array}{l}0.135 \\
1.160 \\
0.005\end{array}$ & $\begin{array}{l}0.229 \\
2.290 \\
0.008\end{array}$ & $\begin{array}{l}0.052 \\
0.440 \\
0.002\end{array}$ & $\begin{array}{l}\text { ND } \\
\text { ND } \\
\text { ND }\end{array}$ & $\begin{array}{l}0.021 \\
0.200 \\
0.001\end{array}$ & $\begin{array}{l}0.081 \\
0.760 \\
0.003\end{array}$ & $\begin{array}{l}\text { NC } \\
\text { NC } \\
\text { NC }\end{array}$ & $\begin{array}{l}0.050 \\
0.560 \\
0.003\end{array}$ \\
\hline
\end{tabular}


TABLE 9-5. PERFORMAKCE TEST DATA SUMARY: ASH ORGAMIC/nETALS (Contimued)

\begin{tabular}{|c|c|c|c|c|c|c|c|c|c|c|c|c|c|c|}
\hline $\begin{array}{l}\text { ST } \\
\text { OP }\end{array}$ & & $\begin{array}{c}\text { INTERM } \\
\text { GOOO } \\
\text { PT }-02\end{array}$ & $\begin{array}{r}\text { MORHAL } \\
\text { POOR } \\
\text { PT-03 }\end{array}$ & $\begin{array}{c}\text { MORMRL } \\
\text { POOR } \\
\text { PT }=04\end{array}$ & $\begin{array}{c}\text { JUTERH } \\
\text { VERY POOR } \\
\text { PT }=05\end{array}$ & $\begin{array}{l}\text { HIGH } \\
\text { POOR } \\
\text { PT-06 }\end{array}$ & $\begin{array}{r}\text { NORMAL } \\
\text { POOR } \\
\text { PT-07 }\end{array}$ & $\begin{array}{r}\text { FORMAL } \\
\text { GOOO } \\
\text { PT }-08\end{array}$ & $\begin{array}{r}\text { NORMAL } \\
\text { GOOO } \\
\text { PT -09 }\end{array}$ & $\begin{array}{r}\text { INTERH } \\
\text { GOOD } \\
\text { PT-10 }\end{array}$ & $\begin{array}{l}\text { NORNAL } \\
\text { GOOO } \\
\text { PT- } 11\end{array}$ & $\begin{array}{r}\text { HIGH } \\
\text { GOOD } \\
\text { PT-12 }\end{array}$ & $\begin{array}{c}100 \\
\operatorname{coc} \\
\mathrm{PT}-13\end{array}$ & $\begin{array}{r}101 \\
\text { PT }=14\end{array}$ \\
\hline HICKEL & $\begin{array}{l}(g / h) \\
\text { (ug/g) } \\
\text { (g/tonne *) }\end{array}$ & $\begin{array}{r}143 \\
1,025 \\
5.20\end{array}$ & $\begin{array}{r}27 \\
214 \\
0.87\end{array}$ & $\begin{array}{r}46 \\
416 \\
1.42\end{array}$ & $\begin{array}{r}117 \\
1,136 \\
4.33\end{array}$ & $\begin{array}{r}21 \\
303 \\
0.78\end{array}$ & $\begin{array}{r}44 \\
381 \\
1.67\end{array}$ & $\begin{array}{r}30 \\
295 \\
1.03\end{array}$ & $\begin{array}{r}54 \\
455 \\
1.77\end{array}$ & $\begin{array}{r}33 \\
361 \\
1.26\end{array}$ & $\begin{array}{r}50 \\
453 \\
1.96\end{array}$ & $\begin{array}{r}27 \\
253 \\
0.96\end{array}$ & $\begin{array}{l}\text { MC } \\
\text { NC } \\
\text { NC }\end{array}$ & $\begin{array}{r}38 \\
432 \\
2.03\end{array}$ \\
\hline ZINC & $\begin{array}{l}(g / h) \\
(u g / g) \\
(g / \text { tonne *) }\end{array}$ & $\begin{array}{r}161 \\
1.153 \\
5.9\end{array}$ & $\begin{array}{r}203 \\
1.639 \\
6.7\end{array}$ & $\begin{array}{r}446 \\
4.248 \\
94.5\end{array}$ & $\begin{array}{r}184 \\
1.789 \\
6.8\end{array}$ & $\begin{array}{r}199 \\
2.798 \\
7.2\end{array}$ & $\begin{array}{r}780 \\
6,727 \\
29.5\end{array}$ & $\begin{array}{r}297 \\
2.171 \\
7.6\end{array}$ & $\begin{array}{r}235 \\
1.994 \\
7.7\end{array}$ & $\begin{array}{r}685 \\
5,330 \\
18.6\end{array}$ & $\begin{array}{r}293 \\
2,664 \\
11.5\end{array}$ & $\begin{array}{r}205 \\
1.930 \\
7.3\end{array}$ & $\begin{array}{l}\text { Ne } \\
\text { NC } \\
\text { HC }\end{array}$ & $\begin{array}{r}145 \\
1.628 \\
7.7\end{array}$ \\
\hline \multicolumn{15}{|c|}{$\begin{array}{l}\text { ECONONI ZER ASH } \\
\text { E. }\end{array}$} \\
\hline $\begin{array}{l}\text { ORGANICS: } \\
\text { TOTAL PCDD }\end{array}$ & $\begin{array}{l}(\mathrm{mg} / \mathrm{h}) \\
\text { (ng/g) } \\
\text { (ug/tonne*) }\end{array}$ & $\begin{array}{l}\text { ND } \\
\text { ND } \\
\text { ND }\end{array}$ & $\begin{array}{l}\text { NO } \\
\text { ND } \\
\text { ND }\end{array}$ & $\begin{array}{l}\text { ND } \\
\text { NO } \\
\text { ND }\end{array}$ & $\begin{array}{r}0.0060 \\
0.43 \\
0.221\end{array}$ & $\begin{array}{r}0.0003 \\
0.03 \\
0.011\end{array}$ & $\begin{array}{l}\text { NO } \\
\text { ND } \\
\text { ND }\end{array}$ & $\begin{array}{r}0.0017 \\
0.09 \\
0.058\end{array}$ & $\begin{array}{r}0.0014 \\
0.09 \\
0.045\end{array}$ & $\begin{array}{l}\text { ND } \\
\text { ND } \\
\text { ND }\end{array}$ & $\begin{array}{l}\text { NO } \\
\text { ND } \\
\text { ND }\end{array}$ & $\begin{array}{l}\text { NO } \\
\text { ND } \\
\text { ND }\end{array}$ & $\begin{array}{l}\text { NC } \\
\text { NC } \\
\text { NC }\end{array}$ & $\begin{array}{l}\text { No } \\
\text { NO } \\
\text { ND }\end{array}$ \\
\hline TOTAL PCDF & $\begin{array}{l}\text { (ng/h) } \\
\text { (ng/g) } \\
\text { (ug/tonne*) }\end{array}$ & $\begin{array}{r}0.0008 \\
0.03 \\
0.029\end{array}$ & $\begin{array}{r}0.0049 \\
0.44 \\
0.159\end{array}$ & $\begin{array}{r}0.0023 \\
0.15 \\
0.076\end{array}$ & $\begin{array}{r}0.02525 \\
1.83 \\
0.931\end{array}$ & $\begin{array}{r}0.0125 \\
1.20 \\
0.456\end{array}$ & $\begin{array}{r}0.0064 \\
0.45 \\
0.242\end{array}$ & $\begin{array}{r}0.0116 \\
0.65 \\
0.403\end{array}$ & $\begin{array}{r}0.0076 \\
0.49 \\
0.249\end{array}$ & $\begin{array}{r}0.0002 \\
0.02 \\
0.008\end{array}$ & $\begin{array}{l}\text { ND } \\
\text { NO } \\
\text { ND }\end{array}$ & $\begin{array}{r}0.0019 \\
0.11 \\
0.066\end{array}$ & $\begin{array}{l}\text { MC } \\
\text { NC } \\
\text { NC }\end{array}$ & $\begin{array}{l}\text { NO } \\
\text { ND } \\
\text { NO }\end{array}$ \\
\hline TOTAL CB & $\begin{array}{l}(\mathrm{mg} / \mathrm{h}) \\
(\mathrm{ng} / \mathrm{g}) \\
\text { (ug/torne") }\end{array}$ & $\begin{array}{l}\text { ND } \\
\text { ND } \\
\text { ND }\end{array}$ & $\begin{array}{l}\text { NO } \\
\text { ND } \\
\text { NO }\end{array}$ & $\begin{array}{l}\text { ND } \\
\text { ND } \\
\text { ND }\end{array}$ & $\begin{array}{l}\text { ND } \\
\text { ND } \\
\text { ND }\end{array}$ & $\begin{array}{l}\text { NO } \\
\text { ND } \\
\text { ND }\end{array}$ & $\begin{array}{l}\text { NO } \\
\text { NO } \\
\text { NO }\end{array}$ & $\begin{array}{l}\text { NO } \\
\text { ND } \\
\text { NO }\end{array}$ & $\begin{array}{l}\text { ND } \\
\text { ND } \\
\text { NO }\end{array}$ & $\begin{array}{l}\text { ND } \\
\text { ND } \\
\text { ND }\end{array}$ & $\begin{array}{l}\text { NO } \\
\text { HO } \\
\text { NO }\end{array}$ & $\begin{array}{l}\text { NO } \\
\text { ND } \\
\text { ND }\end{array}$ & $\begin{array}{l}\text { MC } \\
\text { NC } \\
\text { HC }\end{array}$ & $\begin{array}{l}\text { No } \\
\text { NO } \\
\text { NO }\end{array}$ \\
\hline TOTAL PCB & $\begin{array}{l}(\mathrm{mg} / \mathrm{h}) \\
(\mathrm{ng} / \mathrm{g}) \\
\text { (ug/torne*) }\end{array}$ & $\begin{array}{l}\text { NO } \\
\text { HO } \\
\text { NO }\end{array}$ & $\begin{array}{l}\text { NO } \\
\text { NO } \\
\text { ND }\end{array}$ & $\begin{array}{l}\text { ND } \\
\text { NO } \\
\text { NO }\end{array}$ & $\begin{array}{l}\text { NO } \\
\text { NO } \\
\text { HO }\end{array}$ & $\begin{array}{l}\text { NO } \\
\text { HD } \\
\text { NO }\end{array}$ & $\begin{array}{l}\text { ND } \\
\text { ND } \\
\text { ND }\end{array}$ & $\begin{array}{l}\text { No } \\
\text { ND } \\
\text { ND }\end{array}$ & $\begin{array}{l}\text { NO } \\
\text { ND } \\
\text { NO }\end{array}$ & $\begin{array}{l}\text { ND } \\
\text { ND } \\
\text { HD }\end{array}$ & $\begin{array}{l}\text { NO } \\
\text { ND } \\
\text { ND }\end{array}$ & $\begin{array}{l}\text { NO } \\
\text { ND } \\
\text { ND }\end{array}$ & $\begin{array}{l}\text { NC } \\
\text { NC } \\
\text { NC }\end{array}$ & $\begin{array}{l}\text { No } \\
\text { NO } \\
\text { NO }\end{array}$ \\
\hline TOTAL CP & $\begin{array}{l}\text { (mg/h) } \\
\text { (ng/g) } \\
\text { (ug/tome*) }\end{array}$ & $\begin{array}{r}0.333 \\
14.0 \\
12.1\end{array}$ & $\begin{array}{r}0.045 \\
4.0 \\
1.5\end{array}$ & $\begin{array}{r}0.304 \\
20.0 \\
9.9\end{array}$ & $\begin{array}{r}0.138 \\
10.0 \\
5.1\end{array}$ & $\begin{array}{r}0.042 \\
4.0 \\
1.5\end{array}$ & $\begin{array}{r}0.113 \\
8.0 \\
4.3\end{array}$ & $\begin{array}{l}0 \\
0 \\
0\end{array}$ & $\begin{array}{r}0.216 \\
14.0 \\
7.1\end{array}$ & $\begin{array}{r}0.143 \\
15.0 \\
5.5\end{array}$ & $\begin{array}{r}0.352 \\
26.0 \\
13.8\end{array}$ & $\begin{array}{r}0.170 \\
10.0 \\
6.1\end{array}$ & $\begin{array}{l}\text { KC } \\
\text { NC } \\
\text { NC }\end{array}$ & $\begin{array}{r}0.401 \\
26.0 \\
21.2\end{array}$ \\
\hline TOTAL PAH & $\begin{array}{l}(\mathrm{mg} / \mathrm{h}) \\
\text { (ng/g) } \\
\text { (ug/tome*) }\end{array}$ & $\begin{array}{r}0.29 \\
12 \\
10.4\end{array}$ & $\begin{array}{r}1.5 \\
130 \\
47\end{array}$ & $\begin{array}{r}0.78 \\
51 \\
25\end{array}$ & $\begin{array}{l}6.6 \\
475 \\
242\end{array}$ & $\begin{array}{r}11.4 \\
1,087 \\
413\end{array}$ & $\begin{array}{r}0.76 \\
54 \\
29\end{array}$ & $\begin{array}{r}0.21 \\
12 \\
7.4\end{array}$ & $\begin{array}{r}0.23 \\
15 \\
7.6\end{array}$ & $\begin{array}{l}\text { ND } \\
\text { ND } \\
\text { ND }\end{array}$ & $\begin{array}{r}0.19 \\
14 \\
7.4\end{array}$ & $\begin{array}{l}\text { NO } \\
\text { NO } \\
\text { ND }\end{array}$ & $\begin{array}{l}\text { NC } \\
\text { NC } \\
\text { KC }\end{array}$ & $\begin{array}{l}\text { NO } \\
\text { NO } \\
\text { NO }\end{array}$ \\
\hline $\begin{array}{l}\text { METALS: } \\
\text { ANTIMONY }\end{array}$ & $\begin{array}{l}(g / h) \\
\text { (ug/g) } \\
\text { (g/tonne *) }\end{array}$ & $\begin{array}{r}0.15 \\
6.2 \\
0.0054\end{array}$ & $\begin{array}{r}0.06 \\
5.5 \\
0.0020\end{array}$ & $\begin{array}{r}0.15 \\
10.1 \\
0.0050\end{array}$ & $\begin{array}{r}0.18 \\
12.7 \\
0.0065\end{array}$ & $\begin{array}{r}0.10 \\
9.3 \\
0.0035\end{array}$ & $\begin{array}{r}0.16 \\
11.0 \\
0.0059\end{array}$ & $\begin{array}{r}0.04 \\
2.2 \\
0.0014\end{array}$ & $\begin{array}{l}\text { HO } \\
\text { HD } \\
\text { HD }\end{array}$ & $\begin{array}{r}0.14 \\
14.3 \\
0.0052\end{array}$ & $\begin{array}{r}0.10 \\
7.4 \\
0.0039\end{array}$ & $\begin{array}{r}0.05 \\
2.7 \\
0.0017\end{array}$ & $\begin{array}{l}\text { NC } \\
\text { NC } \\
\text { HC }\end{array}$ & $\begin{array}{r}0.13 \\
B .1 \\
0.0071\end{array}$ \\
\hline ARSEHIC & $\begin{array}{l}(g / h) \\
\text { (ug/g) } \\
\text { (g/tonne }\end{array}$ & $\begin{array}{r}0.28 \\
11.9 \\
0.0103\end{array}$ & $\begin{array}{r}0.15 \\
13.0 \\
0.0047\end{array}$ & $\begin{array}{r}0.19 \\
12.5 \\
0.0062\end{array}$ & $\begin{array}{r}0.20 \\
14.6 \\
0.0074\end{array}$ & $\begin{array}{r}0.18 \\
17.6 \\
0.0067\end{array}$ & $\begin{array}{r}0.15 \\
10.7 \\
0.0057\end{array}$ & $\begin{array}{r}0.16 \\
9.3 \\
0.0057\end{array}$ & $\begin{array}{r}0.17 \\
10.9 \\
0.0055\end{array}$ & $\begin{array}{r}0.12 \\
12.7 \\
0.0047\end{array}$ & $\begin{array}{r}0.18 \\
13.1 \\
0.0070\end{array}$ & $\begin{array}{r}0.21 \\
12.4 \\
0.0075\end{array}$ & $\begin{array}{l}\text { NC } \\
\text { HC } \\
\text { NC }\end{array}$ & $\begin{array}{r}0.24 \\
14.4 \\
0.0127\end{array}$ \\
\hline CAOHIUM & $\begin{array}{l}(g / h) \\
(u g / g) \\
(g / \text { tonne }\end{array}$ & $\begin{array}{r}0.24 \\
10.2 \\
0.0088\end{array}$ & $\begin{array}{r}0.07 \\
6.6 \\
0.0024\end{array}$ & $\begin{array}{r}0.12 \\
7.9 \\
0.0039\end{array}$ & $\begin{array}{r}0.08 \\
5.9 \\
0.0030\end{array}$ & $\begin{array}{r}0.06 \\
6.2 \\
0.0023\end{array}$ & $\begin{array}{r}0.09 \\
6.5 \\
0.0035\end{array}$ & $\begin{array}{r}0.17 \\
9.9 \\
0.0061\end{array}$ & $\begin{array}{r}0.10 \\
6.5 \\
0.0033\end{array}$ & $\begin{array}{r}0.06 \\
5.9 \\
0.0021\end{array}$ & $\begin{array}{r}0.08 \\
5.7 \\
0.0030\end{array}$ & $\begin{array}{r}0.15 \\
8.9 \\
0.0054\end{array}$ & $\begin{array}{l}\text { NC } \\
\text { NC } \\
\text { NC }\end{array}$ & $\begin{array}{r}0.11 \\
6.5 \\
0.0058\end{array}$ \\
\hline
\end{tabular}


TABLE 9-5. PERFORMANCE TEST DATA SUmAARY: ASH ORGAHIC/METALS (Contimued)

\begin{tabular}{|c|c|c|c|c|c|c|c|c|c|c|c|c|c|c|}
\hline \multicolumn{2}{|c|}{$\begin{array}{c}\text { STEAM LOAD } \\
\text { OPERATION } \\
\text { TEST }\end{array}$} & \multirow{2}{*}{$\begin{array}{r}\begin{array}{r}\text { INTERM } \\
\text { GOOD } \\
\text { PT }-02\end{array} \\
\begin{array}{r}5.2 \\
220 \\
0.19\end{array}\end{array}$} & \multirow{2}{*}{$\begin{array}{r}\text { NORMAL } \\
\text { POOR } \\
\text { PT -03 } \\
\begin{array}{r}1.8 \\
160 \\
0.06\end{array}\end{array}$} & \multirow{2}{*}{$\begin{array}{r}\begin{array}{r}\text { NORMAL } \\
\text { POOR } \\
\text { PT }-04\end{array} \\
\begin{array}{r}8.2 \\
540 \\
0.27\end{array}\end{array}$} & \multirow{2}{*}{$\begin{array}{r}\begin{array}{r}\text { INTERM } \\
\text { VERY POOR } \\
\text { PT - O5 }\end{array} \\
\begin{array}{r}4.5 \\
330 \\
0.17\end{array}\end{array}$} & \multirow{2}{*}{$\begin{array}{r}\begin{array}{r}H I G H \\
\text { POOR } \\
\text { PT-06 }\end{array} \\
\begin{array}{r}1.6 \\
150 \\
0.06\end{array}\end{array}$} & \multirow{2}{*}{$\begin{array}{r}\begin{array}{r}\text { MORMAL } \\
\text { POOR } \\
\text { PT - 07 }\end{array} \\
\begin{array}{r}3.1 \\
220 \\
0.12\end{array}\end{array}$} & \multirow{2}{*}{$\begin{array}{r}\begin{array}{r}\text { NORMAL } \\
\text { GOOO } \\
\text { PT - } 08\end{array} \\
2.8 \\
\begin{array}{r}160 \\
0.10\end{array}\end{array}$} & \multirow{2}{*}{$\begin{array}{r}\begin{array}{r}\text { NORHAL } \\
\text { GO00 } \\
\text { PT }-09\end{array} \\
\begin{array}{r}12.8 \\
830 \\
0.42\end{array}\end{array}$} & \multirow{2}{*}{$\begin{array}{r}\text { INTERM } \\
\text { GOOD } \\
\text { PT }-10\end{array}$} & \multirow{2}{*}{$\begin{array}{r}\begin{array}{r}\text { MORMAL } \\
\text { GOOD } \\
\text { PT }-11\end{array} \\
\begin{array}{r}2.8 \\
210 \\
0.11\end{array}\end{array}$} & \multirow{2}{*}{$\begin{array}{r}\begin{array}{r}\text { HIGH } \\
\text { GO00 } \\
\text { PT- } 12\end{array} \\
\begin{array}{r}3.6 \\
210 \\
0.13\end{array}\end{array}$} & \multirow{2}{*}{$\begin{array}{r}\begin{array}{r}\text { LON } \\
\text { OOOD } \\
\text { PT }-13\end{array} \\
\begin{array}{r}\text { NC } \\
\text { KC } \\
\text { NC }\end{array}\end{array}$} & \multirow{2}{*}{$\begin{array}{r}\begin{array}{r}L O N \\
6000 \\
\text { PT-14 }\end{array} \\
\begin{array}{r}5.2 \\
310 \\
0.27\end{array}\end{array}$} \\
\hline CHRONIUM & $\begin{array}{l}(g / h) \\
\text { (ug/g) } \\
\text { (g/tonne ") }\end{array}$ & & & & & & & & & & & & & \\
\hline COPPER & $\begin{array}{l}(g / h) \\
(u g / g) \\
(g / \text { tonne })\end{array}$ & $\begin{array}{r}10.5 \\
440 \\
0.38\end{array}$ & $\begin{array}{r}6.2 \\
550 \\
0.20\end{array}$ & $\begin{array}{r}10.3 \\
680 \\
0.34\end{array}$ & $\begin{array}{l}9.6 \\
679 \\
0.35\end{array}$ & $\begin{array}{l}5.3 \\
509 \\
0.19\end{array}$ & $\begin{array}{l}8.3 \\
590 \\
0.32\end{array}$ & $\begin{array}{l}39.9 \\
2259 \\
1.39\end{array}$ & $\begin{array}{r}28.0 \\
1,820 \\
0.92\end{array}$ & $\begin{array}{r}8.4 \\
880 \\
0.32\end{array}$ & $\begin{array}{r}7.3 \\
540 \\
0.29\end{array}$ & $\begin{array}{r}9.9 \\
580 \\
0.35\end{array}$ & $\begin{array}{l}\text { MC } \\
\text { NC } \\
\text { NC }\end{array}$ & $\begin{array}{l}18.9 \\
1130 \\
1.00\end{array}$ \\
\hline LEAD & $\begin{array}{l}(g / h) \\
(u g / g) \\
(g / \text { tonne }\end{array}$ & $\begin{array}{r}14.0 \\
590 \\
0.51\end{array}$ & $\begin{array}{r}10.4 \\
929 \\
0.34\end{array}$ & $\begin{array}{r}14.3 \\
940 \\
0.47\end{array}$ & $\begin{array}{l}13.1 \\
949 \\
0.48\end{array}$ & $\begin{array}{l}6.9 \\
659 \\
0.25\end{array}$ & $\begin{array}{r}13.8 \\
980 \\
0.52\end{array}$ & $\begin{array}{r}20.1 \\
1,140 \\
0.70\end{array}$ & $\begin{array}{r}11.7 \\
760 \\
0.38\end{array}$ & $\begin{array}{r}9.4 \\
980 \\
0.36\end{array}$ & $\begin{array}{l}11.8 \\
870 \\
0.46\end{array}$ & $\begin{array}{r}16.7 \\
979 \\
0.60\end{array}$ & $\begin{array}{l}\text { NC } \\
\text { NC } \\
\text { NC }\end{array}$ & $\begin{array}{r}15.7 \\
940 \\
0.83\end{array}$ \\
\hline MERCURY & $\begin{array}{l}(\mathrm{mg} / \mathrm{h}) \\
\text { (ug/g) } \\
\text { (mg/torne *) }\end{array}$ & $\begin{array}{l}0.500 \\
0.021 \\
0.018\end{array}$ & $\begin{array}{l}0.390 \\
0.035 \\
0.013\end{array}$ & $\begin{array}{l}\text { ND } \\
\text { ND } \\
\text { ND }\end{array}$ & $\begin{array}{l}0.280 \\
0.020 \\
0.010\end{array}$ & $\begin{array}{l}0.250 \\
0.024 \\
0.009\end{array}$ & $\begin{array}{l}0.300 \\
0.021 \\
0.011\end{array}$ & $\begin{array}{l}0.370 \\
0.021 \\
0.013\end{array}$ & $\begin{array}{l}0.310 \\
0.020 \\
0.010\end{array}$ & $\begin{array}{l}\text { ND } \\
\text { ND } \\
\text { ND }\end{array}$ & $\begin{array}{l}\text { ND } \\
\text { NO } \\
\text { NO }\end{array}$ & $\begin{array}{l}\text { NO } \\
\text { NO } \\
\text { NO }\end{array}$ & $\begin{array}{l}\text { MC } \\
\text { NC } \\
\text { NC }\end{array}$ & $\begin{array}{l}0.670 \\
0.028 \\
0.025\end{array}$ \\
\hline NICKEL & $\begin{array}{l}(g / h) \\
(u g / g) \\
(g / \text { tonne *) }\end{array}$ & $\begin{array}{r}9.8 \\
410 \\
0.35\end{array}$ & $\begin{array}{r}1.5 \\
130 \\
0.05\end{array}$ & $\begin{array}{l}11.7 \\
770 \\
0.38\end{array}$ & $\begin{array}{l}17.8 \\
1289 \\
0.66\end{array}$ & $\begin{array}{l}1.8 \\
170 \\
0.06\end{array}$ & $\begin{array}{r}4.9 \\
290 \\
0.15\end{array}$ & $\begin{array}{r}2.3 \\
130 \\
0.08\end{array}$ & $\begin{array}{r}12.2 \\
790 \\
0.40\end{array}$ & $\begin{array}{r}2.9 \\
300 \\
0.11\end{array}$ & $\begin{array}{r}2.8 \\
210 \\
0.11\end{array}$ & $\begin{array}{r}4.4 \\
260 \\
0.16\end{array}$ & $\begin{array}{l}\text { NC } \\
\text { NC } \\
\text { KC }\end{array}$ & $\begin{array}{r}11.0 \\
660 \\
0.58\end{array}$ \\
\hline ZINC & $\begin{array}{l}(g / h) \\
\text { (ug/g) } \\
\text { (g/tonne *) }\end{array}$ & $\begin{array}{l}22.4 \\
940 \\
0.81\end{array}$ & $\begin{array}{r}16.2 \\
1,449 \\
0.53\end{array}$ & $\begin{array}{r}26.7 \\
1,759 \\
0.87\end{array}$ & $\begin{array}{r}19.4 \\
1.408 \\
0.72\end{array}$ & $\begin{array}{r}18.4 \\
1,758 \\
0.67\end{array}$ & $\begin{array}{r}19.1 \\
1,349 \\
0.72\end{array}$ & $\begin{array}{r}26.5 \\
1,499 \\
0.92\end{array}$ & $\begin{array}{r}30.0 \\
1,950 \\
0.99\end{array}$ & $\begin{array}{l}13.9 \\
1460 \\
0.53\end{array}$ & $\begin{array}{l}31.8 \\
2350 \\
1.25\end{array}$ & $\begin{array}{l}22.9 \\
1349 \\
0.82\end{array}$ & $\begin{array}{l}\text { NC } \\
\text { NC } \\
\text { HC }\end{array}$ & $\begin{array}{l}30.6 \\
1819 \\
1.60\end{array}$ \\
\hline $\begin{array}{l}\text { FABRIC FILTER ASH } \\
\end{array}$ & & & & & & & & & & & & & & \\
\hline $\begin{array}{l}\text { ORGANICS: } \\
\text { TOTAL PCDD }\end{array}$ & $\begin{array}{l}(\mathrm{mg} / \mathrm{h}) \\
(\mathrm{ng} / \mathrm{g}) \\
\text { (mg/tonne *) }\end{array}$ & $\begin{array}{l}\text { NC } \\
\text { NC } \\
\text { NC }\end{array}$ & $\begin{array}{l}\text { NC } \\
N C \\
N C\end{array}$ & $\begin{array}{r}117 \\
84 \\
3.8\end{array}$ & $\begin{array}{r}41 \\
96 \\
1.5\end{array}$ & $\begin{array}{r}281 \\
227 \\
10.2\end{array}$ & $\begin{array}{r}85 \\
154 \\
3.2\end{array}$ & $\begin{array}{r}27 \\
62 \\
0.9\end{array}$ & $\begin{array}{l}147 \\
112 \\
4.8\end{array}$ & $\begin{array}{r}32 \\
27 \\
1.2\end{array}$ & $\begin{array}{r}105 \\
49 \\
4.1\end{array}$ & $\begin{array}{r}6 \\
20 \\
0.2\end{array}$ & $\begin{array}{l}\text { MC } \\
\text { NC } \\
\text { NC }\end{array}$ & $\begin{array}{r}209 \\
186 \\
11.0\end{array}$ \\
\hline TOTAL PCOF & $\begin{array}{l}(\mathrm{mg} / \mathrm{h}) \\
(\mathrm{ng} / \mathrm{g}) \\
\text { (mg/tonne *) }\end{array}$ & $\begin{array}{l}\text { HC } \\
\text { NC } \\
\text { NC }\end{array}$ & $\begin{array}{l}\text { NC } \\
\text { NC } \\
\text { NC }\end{array}$ & $\begin{array}{l}238 \\
172 \\
7.8\end{array}$ & $\begin{array}{r}30 \\
71 \\
1.1\end{array}$ & $\begin{array}{r}349 \\
282 \\
12.7\end{array}$ & $\begin{array}{l}149 \\
271 \\
5.6\end{array}$ & $\begin{array}{r}42 \\
96 \\
1.4\end{array}$ & $\begin{array}{l}292 \\
222 \\
9.6\end{array}$ & $\begin{array}{r}55 \\
47 \\
2.1\end{array}$ & $\begin{array}{l}213 \\
100 \\
8.4\end{array}$ & $\begin{array}{r}18 \\
56 \\
0.6\end{array}$ & $\begin{array}{l}\text { NC } \\
N C \\
N C\end{array}$ & $\begin{array}{l}188 \\
166 \\
9.9\end{array}$ \\
\hline TOTAL CB & $\begin{array}{l}(\mathrm{mg} / \mathrm{h}) \\
(\mathrm{ng} / \mathrm{g}) \\
(\mathrm{mg} / \text { tonne })\end{array}$ & $\begin{array}{l}\text { NC } \\
\text { NC } \\
\text { NC }\end{array}$ & $\begin{array}{l}\text { NC } \\
\text { NC } \\
\text { HC }\end{array}$ & $\begin{array}{r}1,467 \\
1,059 \\
48\end{array}$ & $\begin{array}{r}465 \\
1,085 \\
17\end{array}$ & $\begin{array}{r}2,086 \\
1,684 \\
76\end{array}$ & $\begin{array}{r}518 \\
941 \\
20\end{array}$ & $\begin{array}{r}316 \\
729 \\
11\end{array}$ & $\begin{array}{r}1.667 \\
1.266 \\
55\end{array}$ & $\begin{array}{r}798 \\
684 \\
31\end{array}$ & $\begin{array}{r}1.507 \\
704 \\
59\end{array}$ & $\begin{array}{r}223 \\
708 \\
8\end{array}$ & $\begin{array}{l}\text { NC } \\
\text { NC } \\
\text { NC }\end{array}$ & $\begin{array}{l}1.957 \\
1.727 \\
103\end{array}$ \\
\hline TOTAL PCB & $\begin{array}{l}(m g / h) \\
(n g / g) \\
\text { (mg/torne *) }\end{array}$ & $\begin{array}{l}\text { NC } \\
\text { NC } \\
\text { NC }\end{array}$ & $\begin{array}{l}\text { NC } \\
N C \\
H C\end{array}$ & $\begin{array}{l}\text { ND } \\
\text { ND } \\
\text { HD }\end{array}$ & $\begin{array}{l}\text { ND } \\
\text { ND } \\
\text { ND }\end{array}$ & $\begin{array}{l}\text { ND } \\
\text { ND } \\
\text { HD }\end{array}$ & $\begin{array}{l}\text { ND } \\
\text { ND } \\
\text { ND }\end{array}$ & $\begin{array}{l}\text { NO } \\
\text { NO } \\
\text { ND }\end{array}$ & $\begin{array}{l}\text { NO } \\
\text { ND } \\
\text { ND }\end{array}$ & $\begin{array}{l}\text { NO } \\
\text { ND } \\
\text { ND }\end{array}$ & $\begin{array}{l}\text { ND } \\
\text { KD } \\
\text { HD }\end{array}$ & $\begin{array}{l}\text { NO } \\
\text { ND } \\
\text { NO }\end{array}$ & $\begin{array}{l}\text { NC } \\
\text { NC } \\
\text { NC }\end{array}$ & $\begin{array}{l}\text { NO } \\
\text { KD } \\
\text { HO }\end{array}$ \\
\hline TOTAL CP & $\begin{array}{l}(\mathrm{mg} / \mathrm{h}) \\
(\mathrm{ng} / \mathrm{g}) \\
(\mathrm{mg} / \text { tonne }\end{array}$ & $\begin{array}{l}\text { NC } \\
\text { NC } \\
\text { NC }\end{array}$ & $\begin{array}{l}\text { NC } \\
\text { NC } \\
\text { NC }\end{array}$ & $\begin{array}{r}4,598 \\
3,320 \\
150\end{array}$ & $\begin{array}{r}1,231 \\
2,870 \\
46\end{array}$ & $\begin{array}{r}7,552 \\
6,095 \\
275\end{array}$ & $\begin{array}{r}2,748 \\
4,997 \\
104\end{array}$ & $\begin{array}{r}710 \\
1,636 \\
25\end{array}$ & $\begin{array}{r}5,711 \\
4,336 \\
188\end{array}$ & $\begin{array}{r}2,243 \\
1,924 \\
86\end{array}$ & $\begin{array}{r}4.762 \\
2.225 \\
187\end{array}$ & $\begin{array}{r}456 \\
1.447 \\
16\end{array}$ & $\begin{array}{l}\text { NC } \\
\text { HC } \\
\text { HC }\end{array}$ & $\begin{array}{r}2.511 \\
2.216 \\
133\end{array}$ \\
\hline
\end{tabular}

